Mechanisms of Neisseria gonorrhoeae survival in primary human neutrophils

\author{
Morgan Brittany Johnson \\ Marion, Virginia
}

Bachelors of Science in Biology, The College of William and Mary, 2008

\author{
A Dissertation presented to the Graduate Faculty \\ of the University of Virginia in Candidacy for the Degree of \\ Doctor of Philosophy
}

Department of Microbiology, Immunology, and Cancer Biology

University of Virginia

May, 2014 


\section{$\underline{\text { Abstract }}$}

Neisseria gonorrhoeae (gonococcus, Gc) is the causative agent of the sexually transmitted disease gonorrhea which affects 106 million people each year worldwide. There is currently no protective vaccine against Gc, and due to the rise of antibiotic resistance strains, there is increased necessity for new treatment options. Infection with Gc results in a potent neutrophil (polymorphonuclear leukocyte, PMN)-driven inflammatory response which creates the purulent exudate, a hallmark of gonorrheal infection. PMNs store antimicrobial peptides and proteases in cytoplasmic granules. Upon PMN activation these granules fuse at the plasma or phagosome membrane to release their contents extracellularly or intracellularly, respectively, in order to kill microorganisms. Gc is quite remarkable because despite the variety of mechanisms used by PMNs to kill microbes, viable Gc can be cultured from human gonorrhea exudates and are found intracellularly in PMNs infected ex vivo. These data indicate that Gc has evolved mechanisms to avoid and defend against PMN mediated killing. In this thesis I investigated the mechanisms of Gc intracellular survival in PMNs and determined how one Gc surface structure, opacity-associated (Opa) proteins, affects Gc survival inside PMNs.

Gc has 11 opa genes, encoding for 7-9 distinct Opa proteins. As a result of phase variation during gonorrheal infection, there is a heterogeneous population of $\mathrm{Gc}$ expressing no, one, or multiple Opa proteins. In order to determine how Opa protein expression affects Gc survival in PMNs, we utilized a Gc strain with in frame deletions of all 11 opa genes (Opa- Gc) or a strain constitutively expressing the OpaD protein (Opa+ 
Gc). Consistent with previous observations, we observed that Opa+ Gc have reduced survival in PMNs compared to Opa- Gc. Characterization of the survival defect demonstrated that Opa+ Gc have significantly reduced intracellular survival compared to Opa- Gc. PMNs kill bacteria intracellularly by fusing cytoplasmic granules with bacteria containing phagosomes in order to expose bacteria to oxidative and non-oxidative antimicrobial products. Our data demonstrates the novel finding that Opa+ Gc reside in mature phagosomes, while Opa- Gc phagosomes delay fusion with PMN primary granules. We observed that viable Gc were more likely to reside in immature phagosomes. Additionally, increasing primary granule fusion with Opa- Gc phagosomes was sufficient to decrease intracellular survival, and likewise decreasing primary granule fusion with Opa+ Gc phagosomes was sufficient to increase intracellular survival. In agreement with previous reports, our results indicate that even in the presence of reactive oxygen species, PMN mediated killing of Gc is primarily non-oxidative, specifically due to the activity of phagosomal serine proteases. Opa+ and Opa- Gc display similar sensitivity to PMN non-oxidative antimicrobial components, indicating differences in intrinsic sensitivity to antimicrobial components does not account for the Opa+ intracellular survival defect. From these data we conclude that avoidance of PMN primary granule fusion with $\mathrm{Gc}$ phagosomes is a central mechanism used by Gc to survive inside human PMNs. 


\section{Acknowledgements}

I am deeply grateful to a number of people whose support and guidance has made this dissertation possible. I would like to acknowledge and thank my $\mathrm{PhD}$ mentor, Alison Criss. Alison has been an amazing mentor. Alison offered me priceless guidance and numerous opportunities to grow as a young investigator. She has devoted her time and energy to ensuring my success. Additionally, Alison has been a wonderful friend and colleague. I am truly thankful to have had such a wonderful mentor and scientific role model.

I would also like to thank the members of my thesis committee past and present: Kodi Ravichandran, David Castle, Melissa Kendall, James Casanova, Ian Glomski, and Joanna Goldberg. I appreciate the time they have taken during committee meetings, lab meeting, research in progress presentations, conferences, and casual conversations in the hall to offer advice which has moved my project forward. Their support has been a valuable part of my training experience.

I am also thankful to be part of a wonderful lab which includes Louise Ball, Kylene Daily, Jonathan Handing, Rich Juneau, Stephanie Ragland, and Asya Smirnov. The members of the Criss lab have helped me overcome experimental difficulties and celebrated with me during times of success. They have supported me in every lab meeting, practice presentation, actual presentation, and on a daily basis in the lab. I would like to give a special thanks to Asya Smirnov who has been a valuable mentor to me in the lab and close friend. 
My experience at UVA would not have been the same without my fantastic friendship group. Pursing a $\mathrm{PhD}$ is a long journey and having a strong network of friends has made the journey enjoyable, inspiring, educational, and unforgettable. I would like to give special thanks to Laura Gonyar who has been a true and dedicated friend in every way and in every aspect of my life. I would also like to thank Heather Perry, Meera Murgai, and Ashley Wilson. You were my first friends in graduate school and since day one have greatly impacted and enriched my life!

Finally, I would like to thank my family who has always loved and supported me. My Mom, Granny, Papaw, Chad, and Jacob have guided me throughout my life and are not just an important part of my life but an important part of me. I feel particularly lucky to have a wonderful Mom who has encouraged me to always aim high and dedicated herself to providing me with opportunities to grow and succeed in life. I also feel grateful for my husband, George. George has listened to me, supported me, made me laugh, and grown with me. Any acknowledgement of the support I received while pursuing my $\mathrm{PhD}$ would not be complete without mention of my dog Graham. I would like to thank Graham for his unconditional love and loyalty as well as for waking me up in the mornings and encouraging me to get fresh air and exercise. I am the person I am today because of my amazing family!

Thank you to all! 
Table of Contents

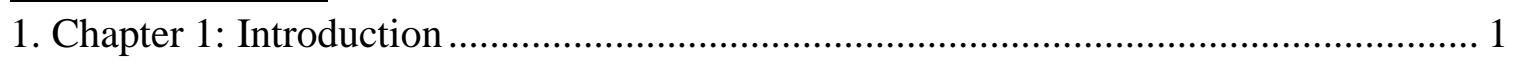

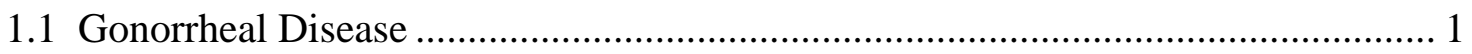

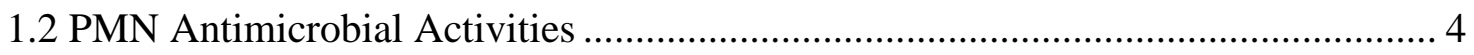

1.3 Model Systems For Examining PMNs During Gc Pathogenesis ............................. 7

1.3.1 The male urethral challenge model. ............................................................. 7

1.3.2 The female murine genital tract model............................................................. 8

1.3.3 Immortalized PMN-like cell lines. .............................................................. 8

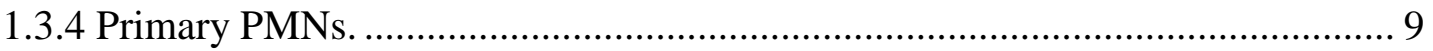

1.4 Gc Survival and Replication in the Presence of PMNs............................................ 10

1.5 Binding and phagocytosis of Gc by PMNs ....................................................... 12

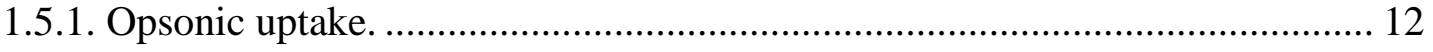

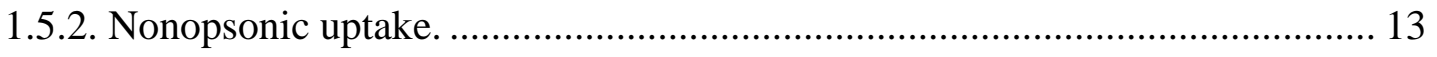

1.6 Gc Defenses Against PMN Antimicrobial Activities ............................................ 17

1.6.1. Defenses against oxidative damage.............................................................. 17

1.6.2. Defenses against non-oxidative damage ......................................................... 22

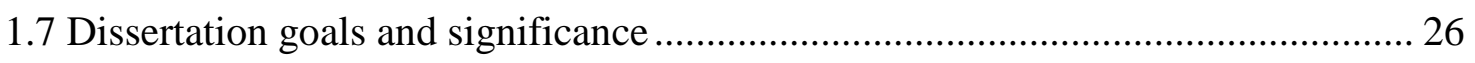

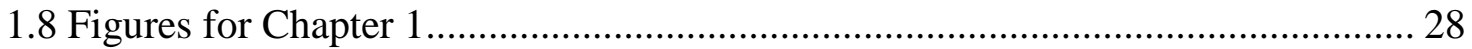

2. Chapter 2: Neisseria gonorrhoeae phagosomes delay fusion with primary granules to enhance bacterial survival inside human neutrophils ......................................................... 33

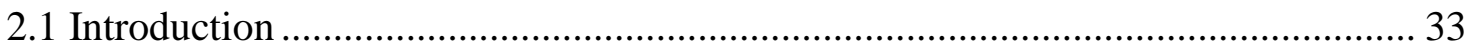

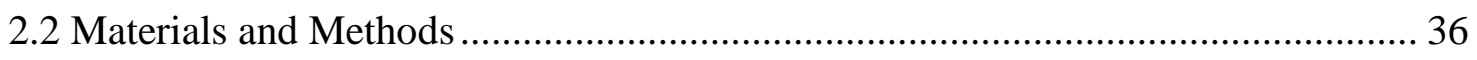

2.2.1 Bacterial strains and growth conditions.......................................................... 36

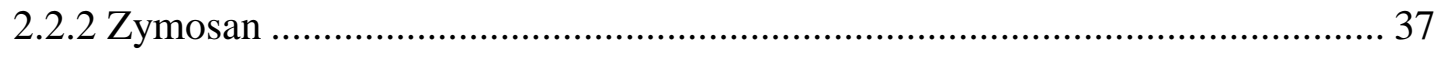

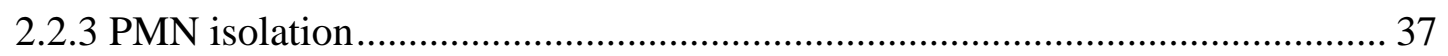

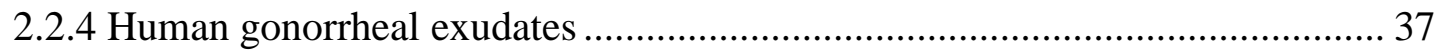

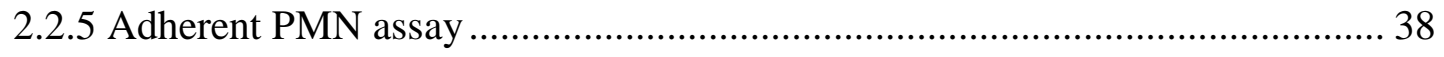

2.2.6 Gc and S. aureus Coinfection: .................................................................. 38

2.2.7 Immunofluorescence ……………….......................................................... 39

2.2.8 Bacterial Viability Dyes ........................................................................ 40

2.2.9 Immunofluorescence for CD63 in combination with bacterial viability dyes.. 41 
2.10 vi

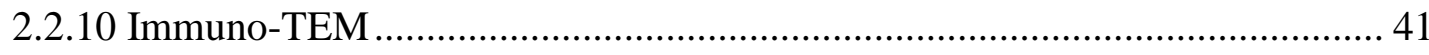

2.2.11 PMN Treatments..................................................................................... 41

2.2.12 Image acquisition, processing, and quantification........................................ 43

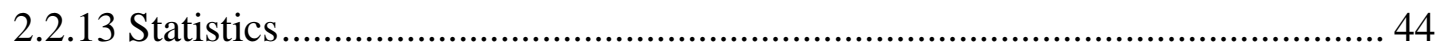

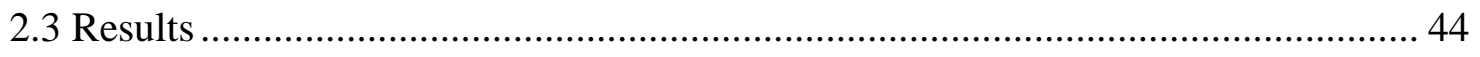

2.3.1 Gc phagosomes in primary human PMNs undergo limited fusion with primary

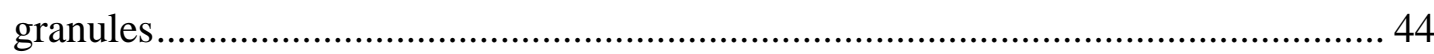

2.3.2 The delay in primary granule fusion with Gc phagosomes does not require active bacterial processes and can be overcome by IgG opsonization ....................... 48

2.3.3 Gc phagosomes devoid of PMN primary granule proteins contain viable Gc . 50 2.3.4 Increasing primary granule fusion with Gc phagosomes reduces internal Gc

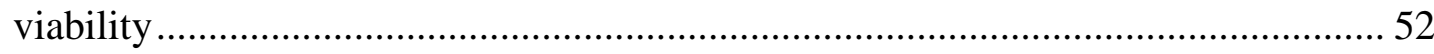

2.3.5 Protease activity contributes to the anti-Gc activity of phagosomes enriched

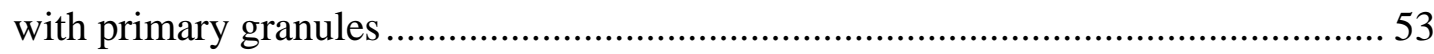

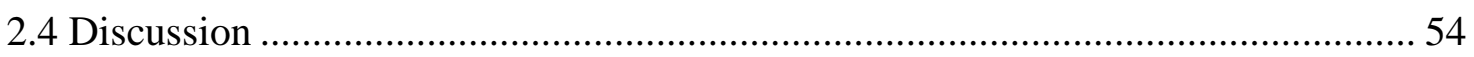

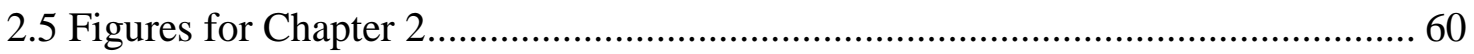

3. Chapter 3: Opa+ Gc have reduced survival in PMNs due to residence in a mature

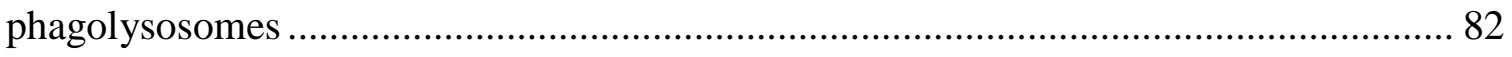

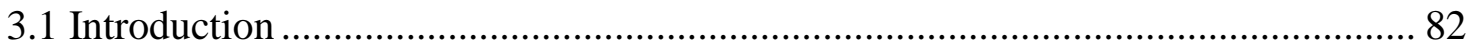

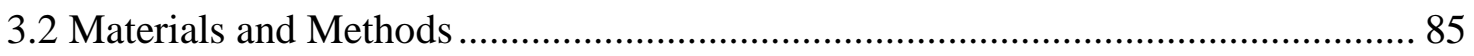

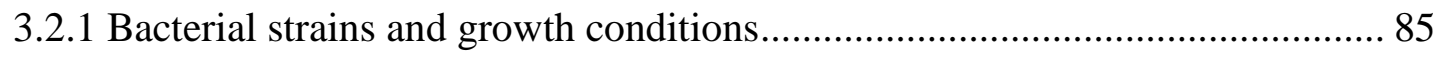

3.2.2 N-domain CEACAM1 Purification ............................................................. 86

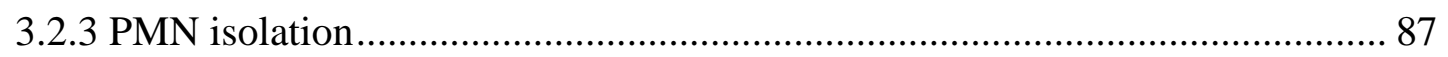

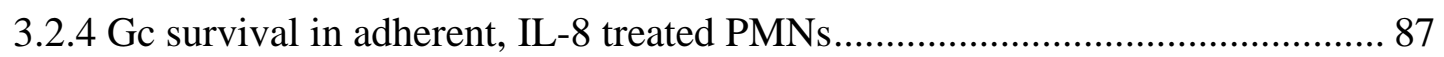

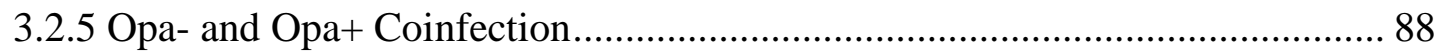

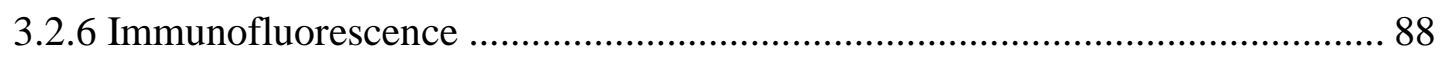

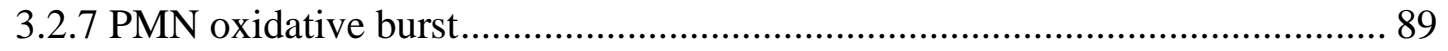

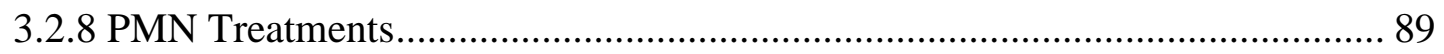

3.2.9 Antimicrobial Protein Susceptibility ........................................................... 90

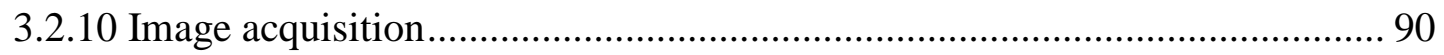

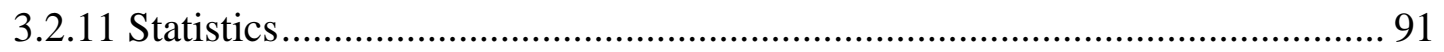


3.3 Resuts $\ldots$ vii

3.3.1 Opa protein expression reduces Gc internal survival in PMNs due to engagement of CEACAM receptors ........................................................................ 91

3.3.2 Increased maturation of phagosomes containing Opa+ Gc ............................. 92

3.3.3 Opa+ Gc residence in degradative phagolysosomes exposes Gc to PMN nonoxidative components which mediate killing ........................................................... 93

3.3.4 Src-family kinase signaling is required for PMNs to direct Opa+ Gc into mature phagolysosomes where they are killed ........................................................ 95

3.3.5 Activation of Src-family kinase signaling increases PMN killing of Opa- Gc 96

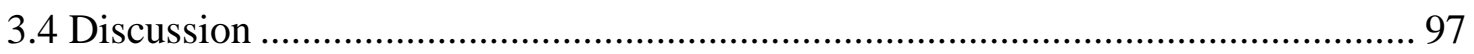

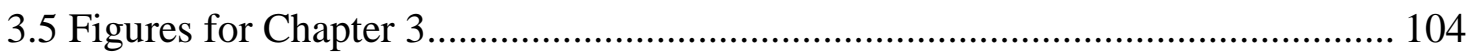

Chapter 4: Discussion and Future Directions ............................................................. 119

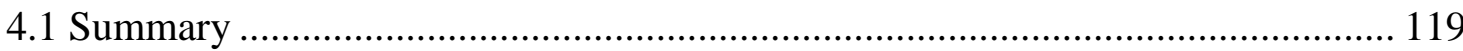

4.2 Route of Internalization of Opa- Gc.............................................................. 120

4.3 PMN granule components with antigonococcal activity...................................... 123

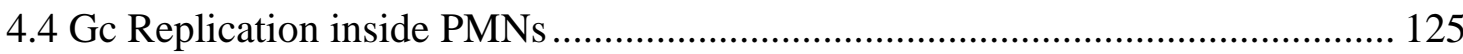

4.5 The role of Opa proteins in Gc interactions with PMNs and during gonorrheal

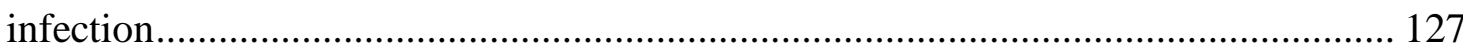

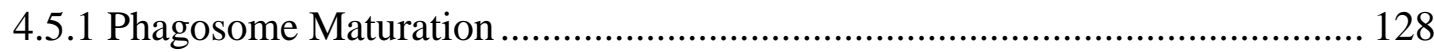

4.5.2 Role of different Opa proteins in PMN activation and Gc survival ............... 130

4.5.3 Advantages and Disadvantages of Opa protein Expression during gonorrheal

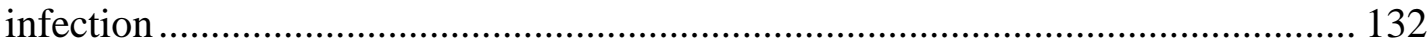

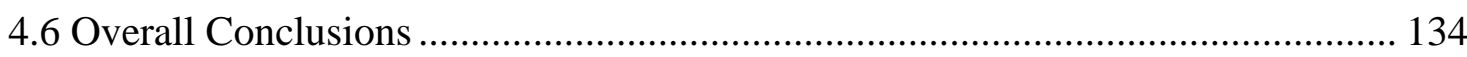

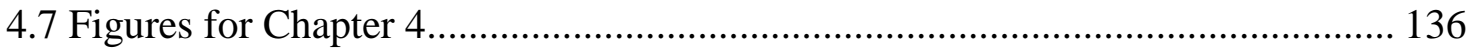

5. Appendix A: Fluorescence microscopy methods for determining the viability of bacteria in association with mammalian cells............................................................ 141

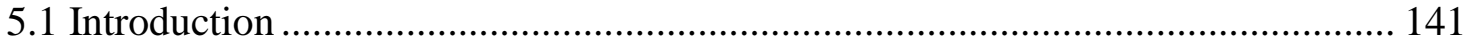

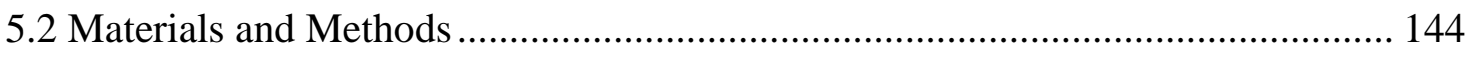

5.2.1 Assessing bacterial viability with propidium iodide and SYTO9................ 144

5.2.2. Assessing bacterial viability with SYTOX Green and DAPI....................... 147

5.2.3. Assessing bacterial viability alongside subcellular localization.................... 148

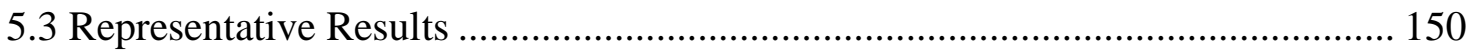


viii

5.4 Discussion ............................................................................................. 154

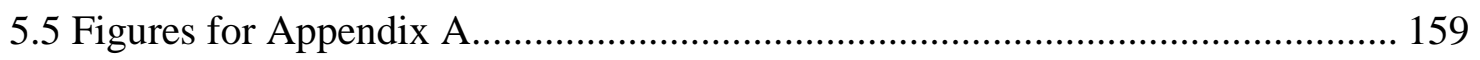

6. Appendix B: Investigation of Gc Replication inside primary human PMNs ............ 163

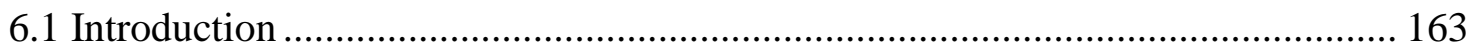

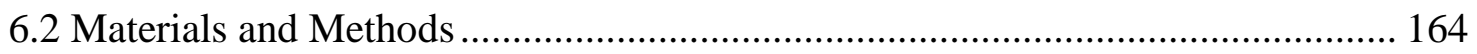

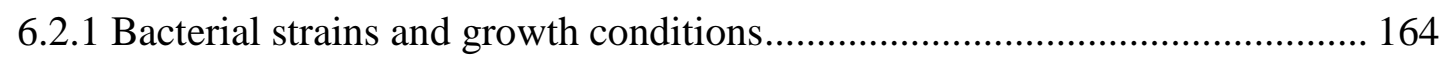

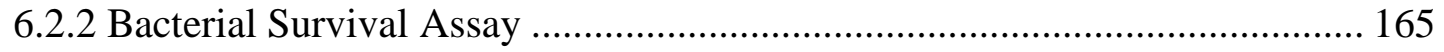

6.2.3 EdU labeling of exponential phase Gc ................................................... 165

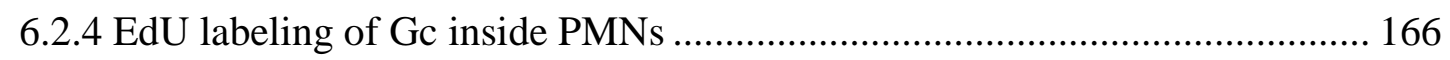

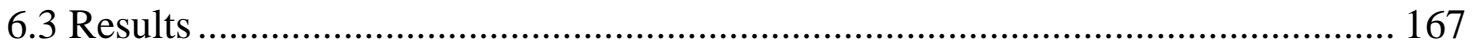

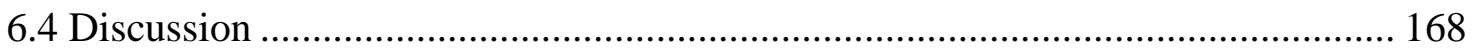

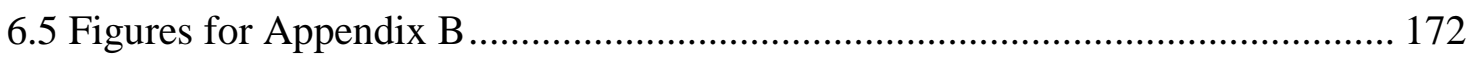

7. Appendix C: Role of pili in Ge survival in PMNs.............................................. 175

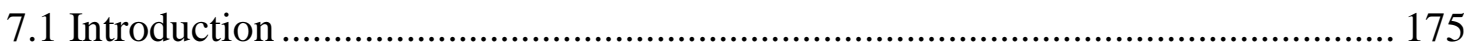

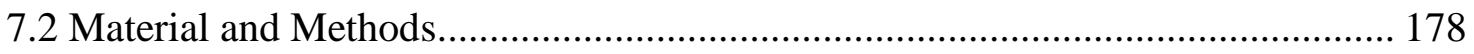

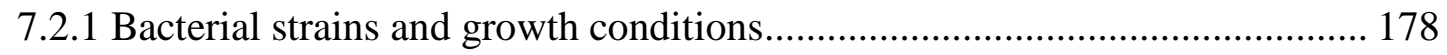

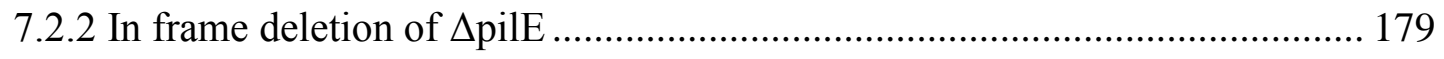

7.2.3 Backcross or introduction of spontaneous deletion of $\Delta$ pilE into FA1090 parent

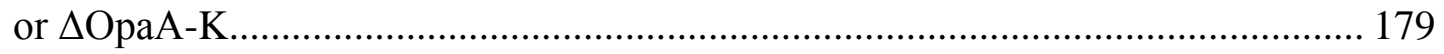

7.2.4 Screening and validation of pilE in frame and large deletions ..................... 179

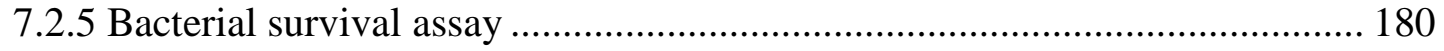

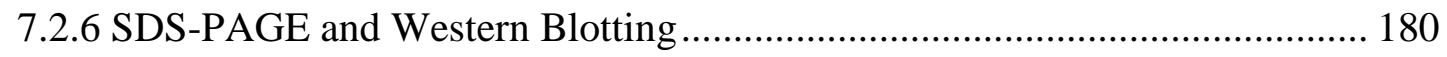

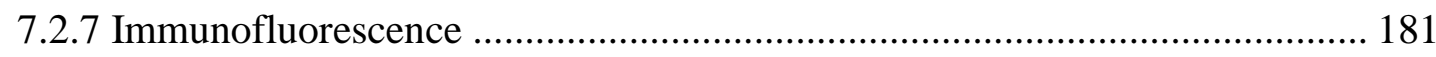

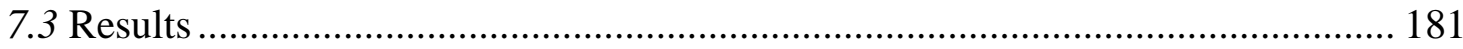

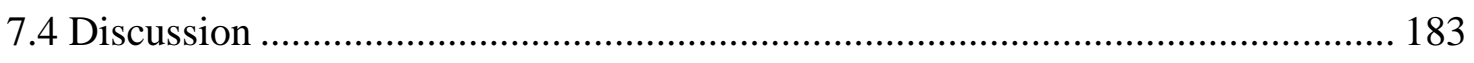

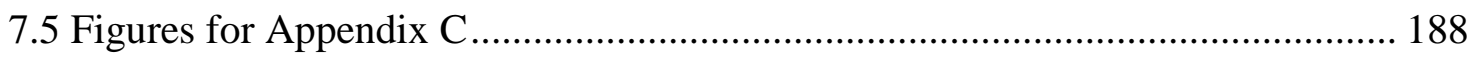

8. Appendix D: PMN granule exocytosis in response to Gc infection ......................... 195

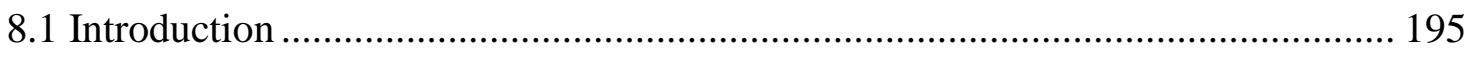

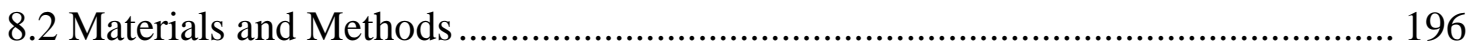

8.2.1 Bacterial strains and growth conditions................................................. 196 


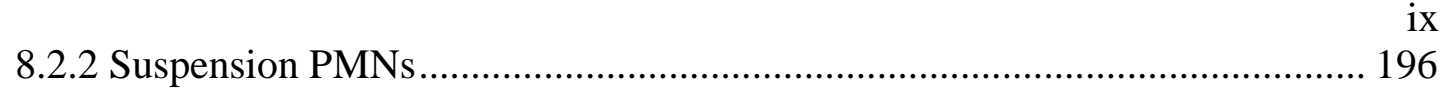

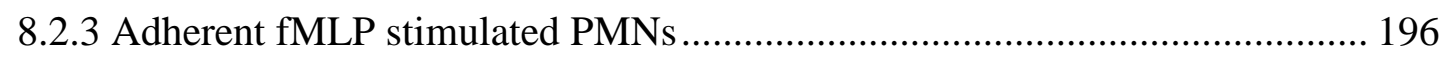

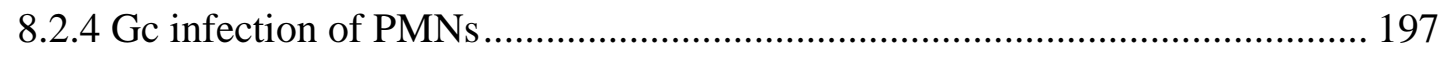

8.2.5 Staining PMNs and Flow cytometry ......................................................... 197

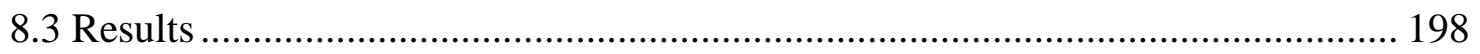

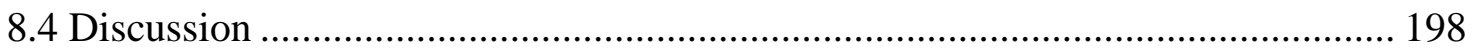

8.5 Figures for Appendix D............................................................................ 202

\section{Table of Figures}

Figure 1: Gonorrhoeal exudates contain numerous PMNs with associated Gc............... 28

Figure 2: Cellular mechanisms of Gc survival after exposure to PMNs. .......................... 29

Figure 3: Opsonic and non-opsonic phagocytosis of Gc by PMNs................................. 31

Figure 4: Mechanisms of Gc survival after exposure to antimicrobial compounds

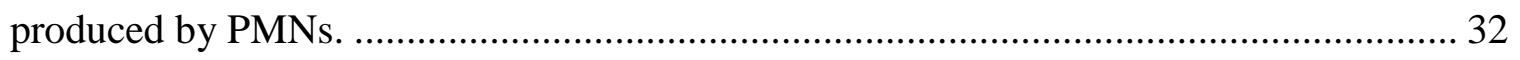

Figure 5: S. aureus and Gc phagosomes fuse with secondary and tertiary granules in

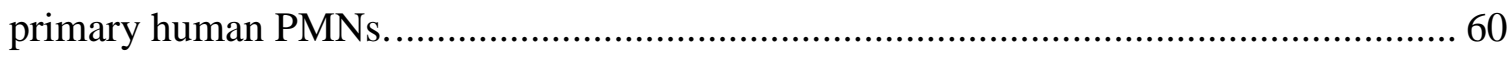

Figure 6: Gc phagosomes undergo less fusion with primary granules than S. aureus

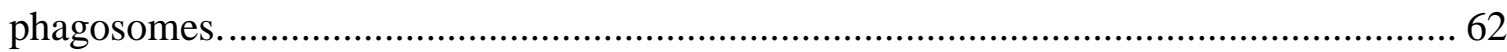

Figure 7: Gc phagosomes in PMNs from gonorrheal exudates have reduced enrichment of

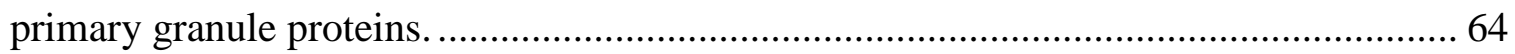

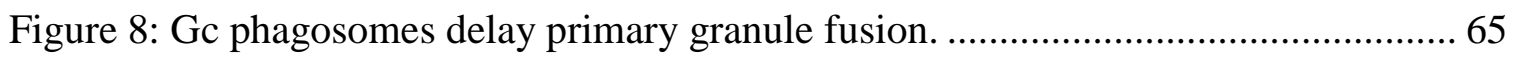

Figure 9: Gc does not globally prevent PMN primary granule fusion. ........................... 67 Figure 10: Active Gc processes are not required to prevent primary granule fusion with

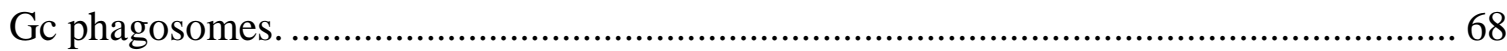

Figure 11: IgG opsonization of Gc increases primary granule fusion with Gc phagosomes.

Figure 12: Titration of anti-Gc polyclonal antibody results in significantly increased trafficking of opsonized Gc to primary granule-positive phagosomes............................ 70 Figure 13: Sytox Green and DAPI effectively discriminate viable and nonviable Gc

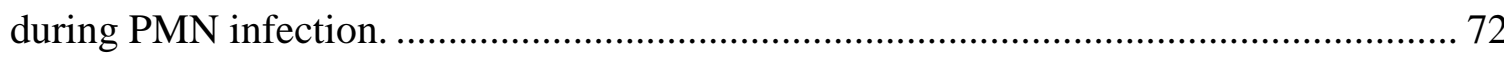

Figure 14: In the absence of fixation, an anti-CD63 antibody effectively labels primary granule and zymosan-containing phagolysosomes. .73

Figure 15: Increased enrichment of the primary granule protein CD63 on phagosomes containing nonviable Gc.

Figure 16: IgG opsonization increases primary granule fusion with Gc phagosomes resulting in decreased Gc intracellular viability. 
Figure 17: LPC treatment of primary human PMNs increases primary granule fusion with

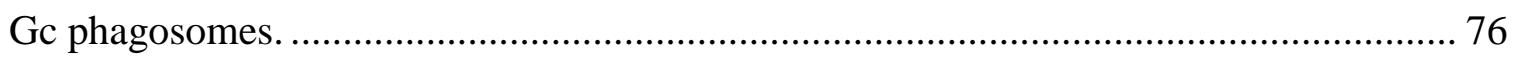

Figure 18: Ionomycin treatment of primary human PMNs increases primary granule

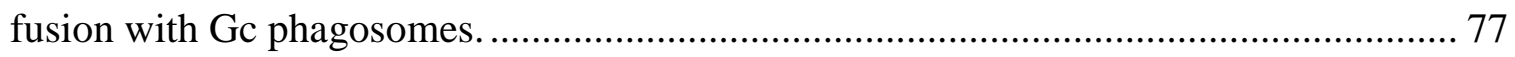

Figure 19: Ectopically increasing primary granule fusion with $\mathrm{Gc}$ phagosomes reduces intracellular Gc viability by a protease-dependent process. ............................................ 78

Figure 20: Protease inhibitor treatment does not affect the LPC-enhanced fusion of primary granules with PMN phagosomes................................................................. 79 Figure 21: Gc IgA protease does not affect primary granule-phagosome fusion in PMNs.

Figure 22: Model for Gc survival inside primary human PMNs................................. 81

Figure 23: Opa+ Gc have reduced survival after exposure to PMNs. ........................... 104

Figure 24: PMN killing of Opa+ Gc is dependent on engagement of CEACAMs. ...... 105 Figure 25: Effect of coating Opa+ Gc with N-CEACAM1 on the PMN oxidative burst

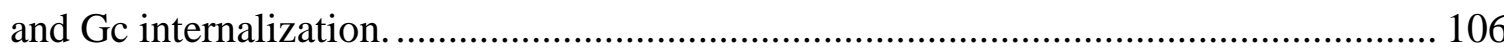

Figure 26: Phagosomes containing Opa+ Gc are significantly more mature than those

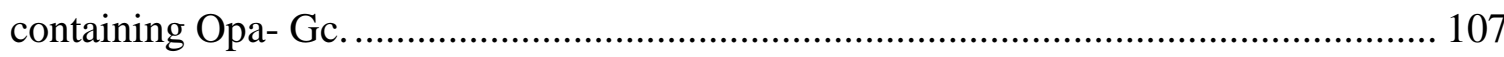

Figure 27: Increased killing of OpaD+ Gc by PMNs is primarily non-oxidative. ......... 109 Figure 28: Inhibition of PMN proteases increases Opa+ Gc survival in PMNs............ 110 Figure 29: Expression of Opa proteins by Gc does not enhance bacterial sensitivity to antimicrobial peptides.

Figure 30: Inhibition of Src-family kinase signaling increases Gc survival in PMNs. .. 112 Figure 31: Effect of CEACAM signaling inhibitors on the PMN oxidative burst and Gc internalization.

Figure 32: Src-family kinase activity is crucial for Opa+ Gc phagosome fusion with primary granules

Figure 33: Activation of Src-family kinase activity decreases Opa- Gc survival in PMNs.

Figure 34: Model for survival of Opa- and Opa+ Gc in PMNs .................................. 118

Figure 35: Essential questions for future studies to address ..................................... 136

Figure 36: SEM image of Gc infected PMNs ........................................................ 138

Figure 37: Advantages for Opa- and Opa+ Gc during gonorrheal infection ................. 139

Figure 38: Lectin labels viable and nonviable Gc ................................................. 159

Figure 39: DNA dyes distinguish viable and nonviable bacteria ................................ 160

Figure 40: DNA dyes identify nonviable bacteria in host cells .................................. 161

Figure 41: DNA dyes distinguish viable and nonviable bacteria in host cells .............. 162

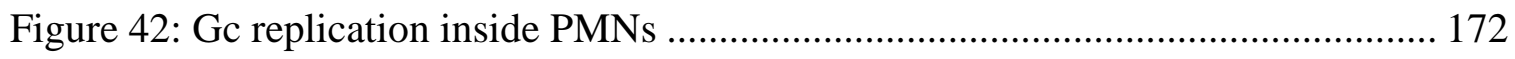

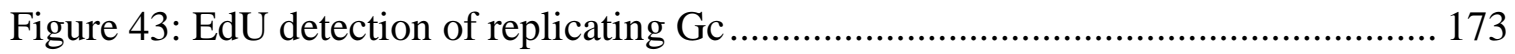

Figure 44: EdU detection of replicating Gc in PMNs............................................... 174

Figure 45: FA1090 spont $\Delta$ pilE are localized to mature phagosomes .......................... 188 
Figure 46: $\triangle \mathrm{OpaA}-\mathrm{K} \Delta$ pilE Gc do not have significantly reduced survival in PMNs compared to $\triangle \mathrm{OpaA}-\mathrm{K}$

Figure 47: FA1090 $\Delta$ pilE Gc do not have a significantly reduced survival in PMNs compared to $\triangle \mathrm{OpaA}-\mathrm{K}$

Figure 48: FA1090 large $\Delta$ pilE and $\Delta$ OpaA-K large $\Delta$ pilE Gc do not have signficiantly reduced survival in PMNs compared to $\triangle \mathrm{OpaA}-\mathrm{K}$ 191

Figure 49: Gc strains used in this study express the 2C7 LOS epitope and PilC 192

Figure 50: PMN adherence stimulates secondary and primary granule exocytosis

\section{$\underline{\text { List of Tables }}$}

Table 1: Antimicrobial components housed in PMN granules..................................... 30

Table 2: Primers used to generate pilE mutants........................................................... 193

Table 3: PCR conditions used to generate and screen pilE mutants.............................. 194 


\section{Chapter 1: Introduction}

This chapter is a modified version of the previously published article, "Resistance of Neisseria gonorrhoeae to neutrophils" [Front. Microbiol., 2:77, 2011.

doi:10.3389/fmicb.2011.00077].

\subsection{Gonorrheal Disease}

Gonorrhea is a major global health problem, with greater than 106 million cases estimated worldwide per year(World Health Organization, 2012). Numbers of reported cases in the United States have remained at approximately 330,000 annually, but it is estimated that the actual number is at least twice as high, and rates of gonorrhea are rapidly increasing among men who have sex with men and young adults (Workowski et al., 2010). The cause of gonorrhea is the Gram-negative diplococcus Neisseria gonorrhoeae (the gonococcus or Gc). Gc is an obligate human pathogen that is transmitted via close sexual contact with an infected individual. Gonorrhea presents as an acute urethritis in men and cervicitis in women, but the pharynx and rectum can also be infected (Wiesner et al., 1980). Because of the frequently asymptomatic nature of female infection, gonorrhea is a major cause of pelvic inflammatory disease, characterized by abdominal pain and tubal scarring that promotes ectopic pregnancy and infertility; untreated infections in men also lead to sterility. Disseminated Gc infections can cause arthritis-dermatitis syndrome, endocarditis, and meningitis. Gc can also be vertically transmitted during childbirth and is still a leading cause of infectious neonatal blindness in the developing world (Wiesner et al., 1980). Gc remains a major public health problem due to rapid acquisition of resistance to multiple antibiotics (Tapsall, 
2009) and its ability to phase and antigenically vary its surface structures, preventing infected individuals from developing a protective immune response and hindering development of a protective vaccine (Virji, 2009).

During gonorrheal disease, Gc uses Type IV pili to initiate colonization at the mucosal epithelium of the urethra, cervix, fallopian tubes, rectum, nasopharynx, and conjunctiva (McGee et al., 1983, Wiesner et al., 1980, Edwards et al., 2004). Tight adherence and internalization of Gc is then mediated by the interaction of opacityassociated proteins (Opa proteins) with carcinoembryonic antigen-related cell adhesion molecules (CEACAMs) and heparan sulfate proteoglycans (HSPGs) on host cells (Kupsch et al., 1993, Makino et al., 1991). Additional Gc factors including lipooligosaccharide (LOS), the cooperative interaction of porin and pili, and the ribosomal protein L12 can aid in the interaction with epithelial cells at specific sites of infection. The terminal lactosamine of lacto-N-neotetraose Gc LOS has been shown to facilitate adherence and internalization into cervical and urethral epithelial cells (Song et al., 2000, Harvey et al., 2001). In urethral epithelial cells, LOS- containing lacto-Nneotetraose can bind the human asialoglycoprotein receptor on urethral epithelial cells, which traffics to the apical cell surface in response to gonococcal infection (Harvey et al., 2001). Additionally, C3b, the ligand for the complement receptor is deposited on the lipid A moiety of Gc LOS. Conversion of $\mathrm{C} 3 \mathrm{~b}$ to $\mathrm{iC} 3 \mathrm{~b}$ followed by the cooperative interaction of porins and pili with the I-domain of complement receptor 3 allows for adherence and invasion of cervical epithelial cells (Edwards et al., 2002b, Edwards et al., 2002a). Finally, Gc can bind the lutropin receptor on endometrial cells via the ribosomal protein L12 (Spence et al., 2000). 
Many of the factors involved in Gc colonization also stimulate the innate immune system via Toll-like receptors (TLRs), NOD-like receptors (NLRs), and C-type lectins found on epithelial cells, macrophages, dendritic cells, and T helper 17 cells (Makepeace et al., 2001, van Vliet et al., 2009, Fisette et al., 2003, Massari et al., 2002, Feinen et al., 2010). TLRs and C-type lectins recognize the Neisserial LOS, porins and the lipoprotein H.8. The TLR4-CD14 complex and C-type lectins recognize LOS while TLR-2 is necessary for recognition of porins and lipprotein H.8 (Massari et al., 2003, Pridmore et al., 2003, Zughaier et al., 2004, van Vliet et al., 2009, Massari et al., 2002, Fisette et al., 2003). Due to differences in receptor stimulation, the cytokine profile released can be different. For example, stimulation of TLR4/CD14 complex by LOS from different strains of Neisseria or by different LOS variants, formed due to phase variation of LOS biosynthetic genes, changes the amount of cytokines released or the cytokine profile, which can skew the T cell immune response (Pridmore et al., 2003, Zughaier et al., 2004, van Vliet et al., 2009). Finally, Gc rapidly turns over peptidoglycan and as a result fragments are shed into the environment. These fragments are then recognized by NLRs on epithelial cells and macrophages (Kaparakis et al., 2010, Mavrogiorgos et al., 2013). Recognition of Gc via TLRs, NLRs, and C-type lectins on host cells results in release of interleukin-8 (IL-8), interleukin-6 (IL-6), tumor necrosis factor (TNF), IL-1 $\beta$, and other cytokines (Fichorova et al., 2001, Waage et al., 1989, Ramsey et al., 1995).

Release of cytokines in response to Gc infection serves to recruit and activate polymorphonuclear leukocytes (neutrophils, PMNs) which promote the inflammatory response characteristic of Gc infection (Figure 1). In men, PMNs appear in urethral swabs and urine several days after infection and immediately prior to the onset of 
symptoms (Cohen et al., 1999). The purulent exudate produced by infected men is the best-known aspect of gonorrheal disease. It is described in the Bible and by Galen, and is reflected in the Greek translation of gonorrhea as "flow of seed" (Edwards et al., 2004). PMNs are also present in the cervical secretions of women with gonorrhea (Evans, 1977). Bacteria in gonorrheal secretions are attached to and within PMNs (Ovcinnikov et al., 1971, Apicella et al., 1996, King et al., 1978a, Evans, 1977, Farzadegan et al., 1975). PMNs are the primary innate immune responders to bacterial and fungal infection, and are capable of phagocytosing and killing a variety of microorganisms (Borregaard, 2010). Despite the presence of numerous PMNs at the site of gonorrheal infection, viable Gc can be cultured from the exudates of infected individuals (Wiesner et al., 1980), and when Gc are exposed to PMNs in vitro, a subset of Gc remain viable (see below). These results show that the PMN-driven innate immune response to Gc is ineffective at clearing Gc. The persistence of Gc in the presence of PMNs facilitates long-term colonization of its obligate human hosts, creating enhanced opportunity for dissemination and transmission.

\subsection{PMN Antimicrobial Activities}

PMNs are the most abundant white cells in the peripheral blood of humans. They are professional phagocytes and the first line of defense of the innate immune system (Borregaard, 2010). In response to peripheral infection or damage, PMNs follow chemotactic cues to extravasate from the bloodstream and migrate through tissues to reach the target site. Mucosal epithelial cells and resident immune cells release cytokines, including interleukin- 8 , interleukin-6, tumor necrosis factor- $\alpha$, and interleukin1 to recruit and modulate PMN function (Borregaard, 2010). These cytokines are released during human Gc infection (Ramsey et al., 1995, Hedges et al., 1998). 
PMNs possess receptors to bind and phagocytose complement- and antibodyopsonized particles (e.g. complement receptor 3 (CR3), Fc receptors (FcRs)). They can also engulf unopsonized particles through lectin-like interactions or using receptors that are specific for ligands on the particle surface (Groves et al., 2008). Interaction between PMNs and a target particle results in the mobilization of different subsets of cytoplasmic granules to the plasma or phagosomal membrane (Figure 2). There are several necessary signals to mobilize and trigger granule fusion, including a rise in intracellular phospholipid signaling, calcium, guanosine triphosphates (GTPases), activation of Srcfamily kinase members, syk tyrosine kinase, and pairing of cognate SNARE complexes (Lacy, 2006, Sengelov et al., 1993). Binding to receptors on the PMN surface results in production of phosphatidylinositol trisphosphate $\left(\mathrm{PIP}_{3}\right)$ from phosphatidylinositol bisphosphate $\left(\mathrm{PIP}_{2}\right)$ due to activation of phophatidylinositol 3-kinase (PI3K) (Arcaro et al., 1993, Koyasu, 2003, Brzezinska et al., 2009). Phospholipase C (PLC) hydrolysis of $\mathrm{PIP}_{2}$ to form IP3 triggers increased levels of intracellular calcium which is sufficient to drive granule release in a concentration dependent manner (Sengelov et al., 1993). Activation of the Rho subfamily of GTPases, particularly Rac2, is necessary for actin cytoskeleton remodeling, which is required for granule mobilization to the target membrane (Werner, 2004, Abdel-Latif et al., 2004, Abdel-Latif et al., 2005). Additionally, receptor engagement results in activation of the Src-family kinase members and Syk. The Src-family members, Hck, Fgr, and Lyn, are selectively recruited to different granule populations. Following activation Hck and Fgr translocate to the primary and secondary granule populations, respectively (Mohn et al., 1995, Gutkind et al., 1989, Mocsai et al., 2000). Syk is recruited to phophorylated ITAMs in activated 
PMNs. In the absence of Syk signaling, PMNs fail to produce an oxidative burst or exocytose granules (Naucler et al., 2002, Van Ziffle et al., 2009, Mocsai et al., 2002). Finally, PMNs express many SNAREs, some which are expressed in PMN granule populations and function to guide docking and fusion of granules with the target membrane (Brumell et al., 1995, Martin-Martin et al., 2000, Mollinedo et al., 2003, Logan et al., 2006).

Granule fusion enables the degradation and killing of microorganisms both intracellularly and extracellularly (Borregaard et al., 2007). PMN mechanisms of microbial killing include production of reactive oxygen species (ROS) via the NADPH oxidase enzyme (the "oxidative burst") as well as the oxygen-independent activities of degradative enzymes and antimicrobial peptides (Table 1). Human PMN granules are classified as azurophilic or primary granules, which contain myeloperoxidase, $\alpha$-defensin peptides, and cathepsin G, among other antimicrobial components; specific or secondary granules containing the subunits of the NADPH oxidase complex gp91 ${ }^{\text {phox }}$ and $\mathrm{p} 22^{\text {phox }}$, LL-37 cathelicidin, lactoferrin, and CR3; and gelatinase or tertiary granules containing gelatinase (Borregaard et al., 2007). PMN granules release their contents in a set order. Initially, gelatinase granule contents degrade extracellular matrix, allowing PMNs to migrate across the tissues underlying the site of infection. Next, the release of specific granules at the target destination increases phagocytic potential due to presentation of CR3 on the PMN surface. Finally, the release of both specific and azurophilic granules creates an environment that is generally hostile to microbial survival (Lacy et al., 2008). PMNs also release neutrophil extracellular traps (NETs) composed of DNA, histones, and selected granule components, which trap and kill microbes without requiring 
phagocytosis (Papayannopoulos et al., 2009). Thus PMNs combine oxygen-dependent and -independent mechanisms to combat intracellular and extracellular microorganisms.

The fact that gonorrheal exudates contain viable Gc indicates that PMNs are ineffective at completely clearing Gc infection. There are two possible mechanisms that could explain how Gc survives PMN challenge: Gc could prevent PMNs from performing their normal antimicrobial functions (phagocytosis, granule content release), or Gc could possess mechanisms to defend against oxidative and non-oxidative components produced by PMNs (Figure 2). As I will discuss, there is substantial evidence for both possibilities, which ultimately enable Gc to survive within a host and be transmitted to new individuals.

\subsection{Model Systems For Examining PMNs During Gc Pathogenesis}

Four experimental approaches have been taken to investigate the involvement of PMNs in gonorrheal disease. Each has contributed to our understanding of how PMNs are recruited during acute infection and how Gc withstands this onslaught.

\subsubsection{The male urethral challenge model. Experimental human infection is}

limited to male urethral inoculation, due to the severe complications of gonorrhea in women such as pelvic inflammatory disease (Cohen et al., 1999). Urethral infection of male volunteers results in the release of chemokines, followed by appearance of PMNs in the urogenital tract 2-3 days after infection, similar to what is seen in natural causes of gonococcal urethritis (Cohen et al., 1999). Exudates from males with experimental Gc infection are morphologically similar to those from natural infections. Electron microscopic analysis of exudates revealed that a subset of Gc inside PMNs appear intact, 
providing the initial evidence that Gc may survive within PMN phagosomes(Farzadegan et al., 1975, Ovcinnikov et al., 1971, Apicella et al., 1996).

1.3.2 The female murine genital tract model. Dr. Ann Jerse (Uniformed Services University of the Health Sciences) has developed a female mouse model of Gc genital tract infection, which allows gonorrheal infection to be examined in a genetically tractable host. Here, estradiol-treated mice are inoculated vaginally with Gc, which allows over $80 \%$ of mice to be colonized with bacteria for over 1 week. Infected mice produce inflammatory cytokines, leading to rapid appearance of PMNs in the genital tract (Jerse, 1999). Experimental infection of female mice has provided insight into the selective advantage of opacity-associated (Opa) protein expression on Gc survival and the roles of Gc virulence factors conferring in vitro resistance to ROS and antimicrobial peptides in in vivo infection (Hobbs et al., 2013, Jerse et al., 2003, Jerse, 1999, Cole et al., 2010, Wu et al., 2006a, Wu et al., 2009, Kunz et al., 2012). Because mice lack the human-specific receptors and other components that are likely to be important for gonorrheal disease, future studies may use mice transgenic for human proteins of interest. Inbred mice that are transgenic for human CEACAMs and CD46, receptors that are implicated in gonorrheal pathogenesis (Merz et al., 2000), have already been developed (Johansson et al., 2003, Gu et al., 2010), with additional mouse strains likely to be produced in the coming years.

1.3.3 Immortalized PMN-like cell lines. The use of immortalized promyelocytic human cell lines to study the molecular mechanisms of Gc pathogenesis provides a system which is readily available, easy to maintain, and easy to manipulate. For example, the HL-60 cell line is a leukemic cell line that can be differentiated into a PMN- 
like phenotype (Collins et al., 1977, Newburger et al., 1979). Differentiated HL-60 cells can phagocytose and generate ROS in response to Opa-expressing Gc akin to primary human PMNs (Pantelic et al., 2004, Bauer et al., 1999). However, HL-60 cells do not possess the robust antimicrobial activity associated with primary cells, due in part to the absence of secondary granules and other vesicular compartments (Le Cabec et al., 1997).

1.3.4 Primary PMNs. Research on the molecular mechanisms underlying Gc infection of PMNs has mostly relied upon primary human cells, purified from freshly isolated human blood. The abundance of PMNs in human blood and the ease of purification make PMNs amenable to infection with Gc in vitro. The limitations of working with primary PMNs include their short half-life, their limited capacity for genetic manipulation, and the person-to-person variability intrinsic to primary human cells. However, primary human PMNs have been used to measure binding and phagocytosis of Gc, quantify Gc survival after PMN exposure, and assess the roles of Gc virulence factors in bacterial defense against PMNs (see below). Gc infection of murine PMNs has also been conducted, although on a more limited basis (Wu et al., 2006a, Soler-Garcia et al., 2007). Future studies using primary human PMNs along with cultured epithelial cells from relevant anatomic sites may provide a means to examine the complex interactions between host cells that occur during gonorrheal infection.

Gc interaction with PMNs is influenced by the physiological state of the PMNs being used. Initial experimentation with primary human PMNs utilized cells and Gc suspended in buffered saline solutions (Rest et al., 1982, Densen et al., 1978), but this is unlikely to reflect the transmigrated, primed state of PMNs in the genitourinary tract during acute infection. Research from the laboratory of Dr. Richard Rest (Drexel 
University) subsequently demonstrated that when PMNs were allowed to adhere to tissue culture-treated dishes, they released their granules and bound significantly more Gc than PMNs in suspension (Farrell et al., 1990). Dr. Michael Apicella's laboratory (University of Iowa) then developed an assay using collagen-adherent PMNs, which generated a system for studying the role of selected Gc virulence factors in bacterial survival after PMN challenge (Simons et al., 2005, Seib et al., 2005). Our laboratory adapted the Apicella protocol to include treatment with the chemokine interleukin-8, which facilitates PMN activation (Borregaard, 2010). We used this system to demonstrate Gc survival inside PMNs and to identify Gc proteins that protect the bacteria from PMNs (Stohl et al., 2005, Criss et al., 2009, Ball et al., 2013, Smirnov et al., 2013, Johnson et al., 2013b).

\subsection{Gc Survival and Replication in the Presence of PMNs}

Although the survival of Gc in association with PMNs was once hotly debated, there is now substantial evidence that gonococci survive and multiply within human phagocytes. Examination of urethral exudates by light and electron microscopy has repeatedly shown the presence of abundant PMNs with associated and internalized Gc (Ovcinnikov et al., 1971, Apicella et al., 1996, King et al., 1978a, Farzadegan et al., 1975). The fact that viable gonococci can be cultured from urethral exudates or cervical swabs is strongly suggestive of Gc survival in the presence of PMNs (Wiesner et al., 1980). In vitro studies from the Apicella laboratory using adherent human PMNs demonstrated that over $50 \%$ of Gc internalized by PMNs remained viable for up to 6 hours, as determined by viable bacterial counts and electron microscopy (Simons et al., 2005). Our group corroborated these findings and directly detected viable extracellular 
and intracellular Gc after PMN infection, using dyes that reveal the integrity of bacterial membranes (Johnson et al., 2013b, Criss et al., 2009). We conclude from these studies that a fraction of Gc can survive both extracellularly and intracellularly in the presence of PMNs.

There is additional evidence that Gc does not just persist within PMNs, but also uses the PMNs as a site for replication. Pioneering studies in the 1970s showed that Gc inside exudate-derived PMNs were sensitive to penicillin, which only kills replicating bacteria. In the presence of antimicrobial agents that cannot permeate eukaryotic membranes, such as spectinomycin or pyocin, numbers of PMN-associated Gc increased over time, indicative of bacterial replication inside exudatous and in vitro-infected PMNs (Casey et al., 1979, Veale et al., 1976, Casey et al., 1980, Veale et al., 1979, Casey et al., 1986). Using electron microscopy and colony counts, the Apicella laboratory observed an increase in Gc within collagen-adherent human PMNs over a six-hour infection, results also suggestive of intracellular replication (Simons et al., 2005). Similarly, we used bacterial viability dyes to observe an increase in the number of viable Gc inside PMNs over time (Criss et al., 2009). While the advantage of Gc replicating inside terminally differentiated cells of a limited life span is questionable, the Apicella group showed that PMNs infected with Gc delay their spontaneous apoptosis (Simons et al., 2006). We anticipate that advances in cellular imaging will provide definitive proof for Gc replication inside PMNs and insight into the timing and extent of this event. 


\subsection{Binding and phagocytosis of Gc by PMNs}

Since gonorrheal secretions contain PMNs associated with viable intracellular and extracellular bacteria, Gc must possess factors that allow for attachment and phagocytosis by PMNs. Opsonic and non-opsonic interactions are the two basic means of phagocytosis, both of which may be utilized by Gc (Groves et al., 2008) (Figure 1).

1.5.1. Opsonic uptake. The two major opsonins for PMN phagocytosis are immunoglobulins and complement, which bind to Fc receptors and complement receptors such as CR3, respectively (Groves et al., 2008). Patients with gonorrhea produce opsonic IgG and IgA directed against Gc surface-exposed components including porin, Opa proteins, pilin, iron-regulated outer membrane proteins, and lipooligosaccharide (LOS) (Schwalbe et al., 1985, Lammel et al., 1985, Brooks et al., 1976, McMillan et al., 1979, Rice et al., 1982, Siegel et al., 1982, Tramont et al., 1980). Intriguingly, serum from individuals with no prior history of gonorrhea contains opsonic IgG that recognizes Gc porin and IgM that reacts against hexosamine-containing LOS isotypes; the non-Gc antigens recognized by these antibodies are not known (Sarafian et al., 1983, Griffiss et al., 1991). Many of the Gc surface structures that promote humoral immune responses are phase and antigenically variable and thus evade antibody-mediated immune surveillance (Virji, 2009). Also, Gc secretes an IgA protease that cleaves the polymeric IgA in mucosal secretions (Blake et al., 1978). Thus complement rather than antibodies is likely to drive the opsonic phagocytosis of Gc by PMNs.

The complement system is a key component of the innate immune system comprised of more than 30 proteins. The complement system can be activated by three 
routes: the classical, the alternative, and the lectin pathway, but all three routes normally proceed to proteolytic activation of the major complement protein C3 and assembly of the membrane attack complex (Ram et al., 2010). Gc has multiple ways of resisting the bactericidal activities of complement in normal human serum. Gc binds the complement regulatory proteins $\mathrm{C} 4 \mathrm{~b}$-binding protein $(\mathrm{C} 4 \mathrm{BP})$ and factor $\mathrm{H}(\mathrm{fH})$ on its surface via porins and sialylated LOS (Gulati et al., 2005, Ram et al., 1998b, Ram et al., 2001, Ram et al., 1998a). C4BP restricts the amount of C3 that can be deposited by the classical complement pathway. $\mathrm{fH}$ is a cofactor for factor I-mediated cleavage of $\mathrm{C} 3 \mathrm{~b}$ to the hemolytically inactive iC $3 \mathrm{~b}$. In the alternative pathway fH irreversibly dissociates factor Bb to limit C3 deposition and subsequent C5 cleavage (Ram et al., 2010). C4BP and fH provide defense against direct complement-mediated killing but concomitantly increase iC3b deposition on the Gc surface. iC3b is a ligand for CR3 (CD11b/CD18). CR3 drives actin-dependent particle engulfment into degradative phagolysosomes and PMN production of ROS (Groves et al., 2008). Although it is assumed that Gc is complementopsonized at mucosal surfaces, how opsonization impacts Gc survival after PMN exposure remains to be explored.

1.5.2. Nonopsonic uptake. In the absence of antibodies or complement, efficient binding and engulfment of Gc by PMNs is achieved via expression of colony opacityassociated (Opa) proteins (Virji et al., 1986, Fischer et al., 1988, King et al., 1978b). Opa proteins, formerly known as "protein II," are a family of closely related, 20-30 kD outer membrane proteins that facilitate Gc binding and internalization by human cells, including PMNs (Sadarangani et al., 2010). Gc strains possess approximately 11 opa genes encoding 7-8 antigenically distinct Opa proteins (Connell et al., 1990, Dempsey et 
al., 1991). Each opa gene is phase-variable due to slipped-strand mispairing in a pentameric nucleotide repeat that places the gene in or out of frame (Murphy et al., 1989). Each opa gene has a different number of copies of the pentameric CTCTT repeat. The repeats are located at the 5' end of the opa gene and code for the hydrophobic residues in the Opa protein signal peptide. Addition or loss of a repeat changes the reading frame and introduces a premature stop codon. The truncated protein lackes the extracellular loops and is non-functional (Stern et al., 1986, Connell et al., 1988, van der Ley, 1988). Changes in the number of pentameric repeats occur during DNA replication. Local DNA denaturation can result in the mispairing of bases in repeat region resulting in insertions or deletions of pentameric repeats (Murphy et al., 1989). As a result of independent phase variation of each individual opa gene, Gc can express zero, one, or any possible combination of Opa proteins. Differential expression of Opa proteins can influence bacterial tropism for host cell types and provides a mechanism of immune evasion (Sadarangani et al., 2010).

Opa proteins bind heparan sulfate proteoglycans (HSPGs) and/or carcinoembryonic antigen-related cellular adhesion molecules (CEACAMs). Only those Opa proteins that bind CEACAMs are reported to influence Gc interactions with PMNs (Sadarangani et al., 2010). The Opa-binding CEACAMs on PMNs are CEACAM1, CEACAM3, and CEACAM6, with CEACAM3 expression exclusively restricted to PMNs. CEACAM1 and CEACAM 3 are transmembrane proteins, while CEACAM 6 possesses a glycosylphosphatidylinositol anchor (Gray-Owen et al., 2006). Binding of Opa proteins to any of the three CEACAMs results in Gc internalization, but via different signaling events (McCaw et al., 2004). CEACAM1 and CEACAM3 have 
immunoreceptor tyrosine-based inhibition and activation motifs, respectively. Therefore, engagement of CEACAM1 triggers phosphatase recruitment, while engagement of CEACAM3 triggers kinase recruitment. Unlike CEACAM1 and CEACAM3, CEACAM6 is glycosylphosphatidylinositol-anchored and the downstream signaling events are largely unknown.

Binding any of these three CEACAMs mediates Gc phagocytosis; however, binding to CEACAM3 triggers increased PMN activation and bacterial killing due to activation of Syk, phosphatidylinositol 3-kinase (PI3K), and Src-family kinases (Sarantis et al., 2007, Sarantis et al., 2012, Buntru et al., 2012, Booth et al., 2003). Recent evidence indicates that the CEACAM receptors on PMNs also function cooperatively. Stimulation of mouse PMNs coexpressing CEACAM1 or CEACAM6 with CEACAM3 resulted in generation of an oxidative burst and degranulation, indicating CEACAM1 and CEACAM6 signals did not inhibit CEACAM3 activation. Additionally, stimulating CEACAM1 and CEACAM3 coexpressing mouse PMNs with a Gc strain that only bound CEACAM1 resulted in PMN activation(Sarantis et al., 2012), suggesting that CEACAM3 can transduce signals via other CEACAM receptors in PMNs. Thus during infection, interaction of Gc with any of the PMN CEACAMs would result in PMN activation, which could lead to Gc killing.

Opa protein expression is selected for in the male urethra, the female cervix during the follicular phase of the menstrual cycle, and in the murine cervix (James et al., 1978, Jerse et al., 1994, Jerse, 1999, Swanson et al., 1988). However, Opa ${ }^{-}$Gc survives better after exposure to PMNs in vitro than isogenic Opa ${ }^{+} \mathrm{Gc}$ (Rest et al., 1982, Virji et 
al., 1986, Criss et al., 2009, Ball et al., 2013). Opa protein expression increases Gc phagocytosis by PMNs and stimulates PMN ROS production and granule exocytosis (Rest et al., 1982, Fischer et al., 1988). In the absence of Opa protein expression, Gc is still internalized by adherent PMNs(Criss et al., 2009, Ball et al., 2013). However, OpaGc fail to assemble the NADPH oxidase complex and do not stimulate reactive oxygen species production(Criss et al., 2008, Smirnov et al., 2013). Differential activation of PMNs due to Opa protein expression or lack of expression may account for Opa+ and Opa- Gc survival differences in PMNs.

Gc surface structures other than Opa proteins may contribute to adherence and phagocytosis by PMNs. Pili and porin cooperatively interact with CR3 on cervical epithelial cells; if this interaction were to occur on PMNs, it could drive Gc internalization by PMNs independent of complement opsonization (Edwards et al., 2002b). In vitro studies suggested that "type 1," virulent, piliated Gc are resistant to phagocytosis and killing by PMNs compared to "type 4," avirulent, non-piliated bacteria (Dilworth et al., 1975, Ofek et al., 1974). We now know that type 1 and type 4 Gc vary in Opa expression as well as piliation, both of which could have contributed to these observations. Purified porins also decrease PMN actin polymerization, which may reduce the phagocytosis of Gc by PMNs (Bjerknes et al., 1995). Serogroup C strains of $N$. meningitidis with lacto-N-neotetraose (LNnT) on LOS are phagocytosed by PMNs in an opsonin-independent manner (Estabrook et al., 1998); it has not been examined whether this LOS epitope on Gc affects phagocytosis by PMNs. Together, the combinatorial expression of Opa proteins, pili, porin, and LOS modulate Gc binding and internalization by PMNs. 


\subsection{Gc Defenses Against PMN Antimicrobial Activities}

Whether they remain extracellular or are phagocytosed by PMNs, Gc must contend with the variety of oxidative and non-oxidative antimicrobial components produced by PMNs (Figure 2). Experiments using Gc isolated directly from human material or guinea pig subcutaneous chamber fluid display increased survival in the presence of phagocytes compared to Gc grown in vitro(Witt et al., 1976, Penn et al., 1977). This suggests Gc possesses factors necessary for defending against phagocyte killing that may be lost or altered when cultured in vitro. These Gc factors provide two approaches to resist the toxic activities of PMNs. First, Gc prevents PMNs from producing or releasing antimicrobial components. Second, Gc expresses virulence factors that defend against these components. Many Gc gene products have been identified that protect Gc from ROS, proteases, or antimicrobial peptides, but in most cases their roles in defense against PMNs have not yet been investigated.

1.6.1. Defenses against oxidative damage. The major species of ROS include superoxide anion, hydrogen peroxide, and hydroxyl radical. These ROS have different reactivities and half-lives, but together they induce DNA, protein, and cell membrane damage that can lead to cell death (Fang, 2004). There are at least four potential sources of oxidative stress for Gc in vivo. 1) PMN NADPH oxidase transports electrons across the phagosomal or plasma membrane to generate superoxide, which spontaneously dismutates to hydrogen peroxide. In PMNs, the primary granule enzyme myeloperoxidase uses hydrogen peroxide as a substrate to generate hypochlorous acid (bleach) (Roos et al., 2003). Phagocytes can also produce reactive nitrogen species (RNS) such as nitric oxide and peroxynitrite, but RNS appear to be of limited importance 
in human PMN antimicrobial activity (Fang, 2004). 2) Enzymes related to phagocyte NADPH oxidase are expressed in epithelial cells, and the survival defect of Gc antioxidant mutants inside primary cervical cells implies that epithelial cells may also be an important source of oxidative stress for Gc (Wu et al., 2006b, Achard et al., 2009, Wu et al., 2005, Potter et al., 2009). 3) Lactobacillus species that generate hydrogen peroxide are normally found in the vaginal flora of women (Eschenbach et al., 1989). Women with inhibitory lactobacilli are less likely to be infected with Gc (Saigh et al., 1978), and lactobacilli inhibit Gc growth in vitro (Saigh et al., 1978, Zheng et al., 1994, St Amant et al., 2002). However, it appears that effects of lactobacilli on Gc may be independent of hydrogen peroxide production, since mucosal secretions can effectively quench lactobacilli-derived ROS (O'Hanlon et al., 2010). 4) Gc generates ROS during aerobic respiration, although this may be less of an issue in vivo, where the oxygen tension in the genitourinary tract is low (Archibald et al., 1986). Gc defenses against oxidative stress involve manipulation of the PMN oxidative burst, detoxifying or repair of oxidative damage, and transcriptional upregulation of antioxidant gene products (Figure 4A).

1.6.1.1. Gc manipulation of the PMN oxidative burst. In the absence of Opa protein expression, Gc fails to induce the PMN oxidative burst (Rest et al., 1982, Virji et al., 1986, Fischer et al., 1988, Criss et al., 2008). Even in the presence of $\mathrm{Opa}^{+} \mathrm{Gc}$ that induce ROS production in PMNs, the magnitude of ROS production is small relative to stimuli such as phorbol esters or other bacteria (Simons et al., 2005). Gc utilizes three mechanisms to reduce the amount of ROS produced by PMNs. First, exposure to lactate that is released from PMNs undergoing glycolysis stimulates the rate of Gc oxygen 
consumption, reducing the amount available to PMNs as a substrate for NADPH oxidase (Britigan et al., 1988). Second, purified Gc porin inhibits PMN ROS production in response to Gc, yeast particles, and latex beads (Lorenzen et al., 2000), but not formylated peptides (Haines et al., 1988, Bjerknes et al., 1995). Whether porin has this effect in the context of whole Gc bacteria remains to be examined. Third, we reported that Opa ${ }^{-}$Gc suppresses the PMN oxidative burst induced by serum opsonized staphylococci and formylated peptides by a process requiring bacterial protein synthesis and bacteria-PMN contact (Criss et al., 2008); the bacterial products mediating this effect are not known at this time.

\subsubsection{Detoxification and repair of oxidative damage. Bacteria respond to}

oxidative stress by catalysis of superoxide to hydrogen peroxide by superoxide dismutase (SOD), which is then converted to water and molecular oxygen by catalases and peroxidases (Seib et al., 2006). Gc possesses a single cytoplasmic superoxide dismutase (SodB), one cytoplasmic catalase (KatA), and several genes annotated as peroxidases. SodB activity is low in Gc and does not play a significant role in protection against oxidative stress (Tseng et al., 2001). In comparison, KatA is crucial to Gc defense against ROS. Gc has approximately 100 fold higher levels of catalase than E. coli (Hassett et al., 1990). Disruption of katA significantly reduces Gc survival to hydrogen peroxide and superoxide in vitro (Soler-Garcia et al., 2004, Stohl et al., 2005, Johnson et $a l ., 1993$ ) and reduces the survival of some strains of Gc in the female murine genital tract (Wu et al., 2009). Gc also has high peroxidase Gc activity due to the periplasmic cytochrome c peroxidase encoded by ccp (Archibald et al., 1986). ccp mutant Gc show slight sensitivity to hydrogen peroxide, which is markedly enhanced when katA is also 
inactivated (Turner et al., 2003). Gc also imports $\mathrm{Mn}$ (II) into its cytoplasm via the MntABC transporter, where it scavenges superoxide and hydrogen peroxide by a mechanism independent of SodB and catalase (Tseng et al., 2001). This system is similar to the manganese transport system in Lactobacillus plantarum (Archibald et al., 1984).

Gc can also repair oxidative damage to proteins and DNA. Gc expresses two forms of methionine sulfoxide reductase, which reverses the oxidation of methionine residues in proteins. The MsrA protein is localized to the cytoplasm, while MsrB is secreted to the outer membrane. A msrAB mutant is more sensitive to hydrogen peroxide and superoxide in vitro than its wild-type parent (Skaar et al., 2002). Many Gc gene products involved in recombinational DNA repair, base excision repair, and nucleotide excision repair participate in Gc defense against ROS, such as the DNA-binding protein RecN (Stohl et al., 2006, Davidsen et al., 2005, LeCuyer et al., 2010). The putative metalloprotease Ngo1686 has DD-carboxypeptidase and endopeptidase activities to cleave peptidoglycan (Stohl et al., 2012)and functions in pilus biogenesis(Stohl et al., 2013). Ngo1686 helps protect Gc from hydrogen peroxide and the lipid oxidant cumene hydroperoxide (Stohl et al., 2005, Stohl et al., 2013). Both $n g o 1686$ and recN mutants have significant survival defects after exposure to primary human PMNs, but a mutant in the recombinational repair gene recA does not (Stohl et al., 2005, Criss et al., 2009, Stohl et al., 2013).

1.6.1.3. Transcriptional induction of antioxidant gene products. Gc pre-exposed to hydrogen peroxide survives PMN challenge significantly better than unexposed Gc (Criss et al., 2009). This finding implies that Gc possesses complex transcriptional 
circuitry that is important for defenses against ROS and/or PMNs. The transcriptome of Gc exposed to sublethal concentrations of hydrogen peroxide was defined and revealed the upregulation of antioxidant genes as well as RecN and Ngo1686 after oxidative challenge (Stohl et al., 2005). Antioxidant gene expression is regulated by selected transcriptional repressors. The OxyR protein represses KatA expression, which is relieved following oxidative stress in order to increase catalase production (Tseng et al., 2003). PerR is responsive to $\mathrm{Mn}(\mathrm{II})$ levels and represses expression of MntC, part of the Mn(II) transporter (Wu et al., 2006b). Finally, Ngo1427, a LexA homolog, represses expression of RecN, which is relieved when a cysteine residue is oxidized (Schook et al., 2011).

\subsubsection{PMNs primarily direct non-oxidative antimicrobial components against}

Gc. Although Gc has complex mechanisms for detecting oxidative damage and responding to it, the importance of these processes in Gc survival to PMNs appears to be limited. Mutants in $k a t A$, $s o d B, c c p$, or $m n t A B C$, alone or in combination, do not affect the percentage of Gc that can survive PMN challenge (Seib et al., 2005, Criss et al., 2009). Moreover, Gc survival is similar between normal PMNs and ROS-deficient PMNs obtained from patients with chronic granulomatous disease (CGD) (Rest et al., 1982, Criss et al., 2008), and PMNs maintained in anoxic conditions, as are likely to be found in the upper reproductive tract, are not impaired for antigonococcal activity (Casey et al., 1986, Frangipane et al., 1992). Our group showed that Gc survival was unaffected after exposure to PMNs treated with diphenylidene iodonium (DPI), an inhibitor of NADPH oxidase. DPI treatment or CGD PMNs did not increase the percent survival of ngo1686 or recN Gc, nor did it enhance survival of $\mathrm{Opa}^{+} \mathrm{Gc}$ that induce ROS from 
PMNs (Criss et al., 2009). From these results, we conclude that PMNs primarily direct non-oxidative antimicrobial activities against Gc. The functional redundancy in Gc antioxidant defenses may be sufficient to counter PMN-derived ROS; alternatively, PMNs may not generate enough ROS during infection to affect Gc survival.

1.6.2. Defenses against non-oxidative damage. Seminal research from the Rest and Shafer laboratories indicated that components found inside PMN granules display oxygen-independent antigonococcal activity (Rest, 1979, Casey et al., 1985, Rock et al., 1988). These components include the bactericidal/permeability-increasing protein (“hCAP57”), cathepsin G protease, and LL-37 antimicrobial peptide (Casey et al., 1985, Shafer et al., 1986b, Shafer et al., 1998). Unlike many Gram-negative bacteria, Gc are highly resistant to another class of antimicrobial peptides, the defensins (Qu et al., 1996), although the observed resistance may depend on experimental conditions (Porter et al., 2005). Of PMN non-oxidative granule components, cathepsin G and LL-37 have been the most actively studied for their effects on Gc.

Cathepsin $\mathrm{G}$ is a highly cationic serine protease that resides in PMN primary granules. It enzymatically cleaves Gc outer-membrane proteins including porin and Opa proteins (Shafer et al., 1987, Rest et al., 1981). However, heat and protease inhibitors do not impede cathepsin G's ability to kill Gc in vitro, indicating its antigonococcal activity is independent of its function as a protease (Shafer et al., 1986b). Cathepsin G can insert into Gc membranes, but killing does not appear to be due to changes in membrane permeability; instead, cathepsin G may impede peptidoglycan biosynthesis (Shafer et al., 1990). 
LL-37 is the active form of an $18 \mathrm{kD}$ protein precursor ("hCAP18") that resides in secondary granules. hCAP-18 is proteolytically processed to LL-37 by the primary granule protein proteinase-3 (Sorensen et al., 2001). hCAP-18/LL-37 is also synthesized by mucosal epithelial cells, and is readily detected in cervicovaginal secretions (mean LL-37 concentration of $10 \mu \mathrm{g} / \mathrm{ml}$ ) and seminal plasma (mean hCAP-18 concentration of $86 \mu \mathrm{g} / \mathrm{ml}$ ) (Tjabringa et al., 2005, Malm et al., 2000). Gc infection increases the levels of hCAP18/LL-37 by two- to four-fold in cervical and urethral washes (Tjabringa et al., 2005, Porter et al., 2005). These concentrations of LL-37 would be sufficient to exert antibacterial activity on Gc; the mean inhibitory concentration of LL-37 for Gc is $6 \mu \mathrm{g} / \mathrm{ml}$ (Shafer et al., 1998). The antigonococcal mechanism of action of LL-37 remains enigmatic but may be related to its ability to form pores that disrupt the integrity of bacterial membranes (Brogden, 2005).

Although Gc are susceptible to cathepsin G and LL-37 in vitro, the ability of some percentage of Gc to survive PMN exposure suggests that the bacteria have evolved mechanisms to counter these antimicrobial components. These mechanisms involve direct modulation of PMN granule release, changes to the Gc surface to resist nonoxidative antimicrobial components, and active export of these components (Figure 4B).

1.6.2.1. Modulation of PMN granule release. Both pili and porin have been reported to reduce PMN granule fusion with the plasma membrane or phagosomes. When added to primary PMNs, purified porins inhibit primary and secondary granule exocytosis (Bjerknes et al., 1995, Lorenzen et al., 2000). “Type 1,” piliated Gc was also reported to inhibit primary granule exocytosis relative to "type 4," nonpiliated Gc, but 
additional surface structures expressed on type 1 bacteria may have mediated this result (Densen et al., 1978). More detailed studies with isogenic Gc strains are necessary to determine whether and how Gc surface structures influence granule mobilization.

\subsubsection{Modifications to the Gc surface. Gc LOS is thought to mask proteins} which are degraded by cathepsin G, since truncation or loss of LOS results in increased binding of cathepsin $\mathrm{G}$ and increased susceptibility to cathepsin G-mediated killing (Shafer, 1988). Two modifications of LOS impact bacterial interactions with host cells and host defenses: phosphoethanolamine (PEA) substitution on lipid A or the oligosaccharide, and sialylation of the terminal Gal $\beta 1-4 \mathrm{GlcNAc}$ epitopes of the oligosaccharide (Plested et al., 1999, Mandrell et al., 1990). PEA addition to the heptose group on the beta chain of the core oligosaccharide enhances Gc serum resistance but does not affect susceptibility to antimicrobial peptides (Lewis et al., 2009). In contrast, PEA addition to lipid A by the LptA enzyme increases resistance to both normal human serum and cationic antimicrobial peptides, indicating that structural changes in LOS contribute to the ability of gonococci to resist the bactericidal action of these innate immune components (Lewis et al., 2009). In the related bacterium N. meningitidis, expression of $l p t A$ is positively regulated by the $m i s R / m i s S$ two-component regulatory system (Tzeng et al., 2008, Newcombe et al., 2005). The roles of MisR/MisS and LptA in Gc pathogenesis, particularly in survival after exposure to PMNs, remain to be examined. The gonococcal $\alpha$-2,3-sialyltransferase Lst transfers sialyl groups from hostderived CMP-N-acetylneuraminic acid to the terminal galactose residue on the oligosaccharide of LOS (Gilbert et al., 1996). Sialylation contributes to Gc resistance to normal human serum as well as PMN-derived oxygen-independent antimicrobial factors 
(Shafer et al., 1986b, Parsons et al., 1992). Importantly, sialylated Gc are more resistant to PMNs in vitro, and sialylation contributes to Gc survival in the murine female genital tract (Wu et al., 2006a, Rest et al., 1992, Gill et al., 1996, Kim et al., 1992). Whether resistance to PMNs is due to resistance to PMN-derived oxygen-independent factors or to other aspects of Gc-PMN interactions, such as binding and phagocytosis, remains to be explored. In addition to LOS, changes in other surface components may contribute to Gc resistance to non-oxidative antimicrobial factors. For instance, loss of Opa expression enhances Gc resistance to serine proteases (Cole et al., 2010, Blake et al., 1981), and $N$. meningitidis lacking pili (due to insertional mutagenesis of the pilMNOPQ operon) are more resistant to the model antimicrobial peptide polymyxin B (Tzeng et al., 2005).

\subsubsection{Gc export of antimicrobial components. The multiple transferable} resistance (mtr) locus is a key determinant of Gc resistance to antimicrobial agents (Shafer et al., 1998). Mtr, a member of the Resistance-Nodulation-cell-Division (RND) family of efflux pumps, is encoded by a three gene operon designated $m \operatorname{tr} C D E$. MtrC spans the periplasm to link the inner membrane protein $\mathrm{MtrD}$, the multidrug efflux transporter, with outer membrane protein MtrE, the channel for export of antimicrobials to the extracellular environment (Hagman et al., 1995a). MtrCDE uses the proton motive force to export a variety of compounds from the Gc cytoplasm, including antibiotics, detergents, and antimicrobial peptides (Hagman et al., 1995a, Veal et al., 2002). mtrCDE is negatively regulated by the MtrR transcriptional repressor (Hagman et al., 1995b) and positively regulated by the MtrA transcriptional activator (Rouquette et al., 1999). Mutations in $m t r R$ and $m t r A$ that modulate expression of MtrCDE affect Gc resistance to antimicrobial peptides (Hagman et al., 1995a, Hagman et al., 1995b). MtrCDE 
expression promotes Gc survival in the murine female genital tract (Jerse et al., 2003) and enhances resistance to murine antimicrobial peptides (Warner et al., 2008). Since MtrCDE protects Gc from host-derived antimicrobial compounds and contributes to Gc survival in vivo, it is a reasonable hypothesis that MtrCDE is important for Gc survival after exposure to PMNs, but this remains to be tested. Gc also uses the FarAB efflux pump system to confer resistance to long chain fatty acids, independent of Mtr activity (Lee et al., 1999). The Far system is composed of the FarA membrane-spanning linker, the FarB cytoplasmic membrane transporter, and MtrE. Far expression is believed to be important for survival of isolates at the rectal mucosal surface, which is rich in dietderived fatty acids, and does not contribute to Gc survival in the murine genital tract (Jerse et al., 2003). The contribution of the Far system to defense against PMNs, which may release fatty acids (Huang et al., 2010), is yet to be explored.

\subsection{Dissertation goals and significance}

Despite the prevalence of gonorrhea in the human population and the abundance of PMNs during acute gonorrheal disease, we are just beginning to understand the molecular mechanisms underlying Gc interactions with PMNs and resistance to PMNmediated killing. The goal of this dissertation is to address two questions that remain in the field. First, how does Gc persist over time inside PMNs? Previous studies from our lab and others indicate a fraction of Gc remain viable in PMNs (Simons et al., 2005, Criss et al., 2009). Electron microscopic analysis of Gc-infected PMNs indicates that viable bacteria are found within phagosomes (Simons et al., 2005, Criss et al., 2009). Many intracellular pathogens manipulate phagosome maturation or the phagosome environment to create a niche conducive for survival (Amer et al., 2002, Urban et al., 
2006). Characterizing the Gc phagosome will determine if Gc manipulate phagosome maturation to remain viable in PMNs. Additionally, understanding the phagosome environment will provide insight into the antimicrobial components Gc must combat against in order to remain viable in PMNs.

Although virulence-associated Gc surface structures such as Opa proteins, pili, porin, and LOS have been highly investigated, their effects on Gc survival inside PMNs remain enigmatic. The second question addressed in this dissertation is, how does Gc expression of CEACAM-binding Opa proteins affect Gc survival in PMNs? Despite selection for Opa protein expression in men with symptomatic gonorrhea, Opa+ Gc have reduced survival in PMNs compared to Opa- Gc (Virji et al., 1986, Ball et al., 2013, Criss et al., 2009, Rest et al., 1982). Here I will investigate the role of Opa protein expression in PMN activation and in susceptibility to PMN antimicrobial components, both of which may contribute to reduced survival in PMNs. Understanding how Gc structures such as Opa proteins affect Gc interactions and survival in PMNs may shed light onto mechanisms used by Gc to defend against PMN mediated killing, a critical aspect of Gc pathogenesis. 


\subsection{Figures for Chapter 1}

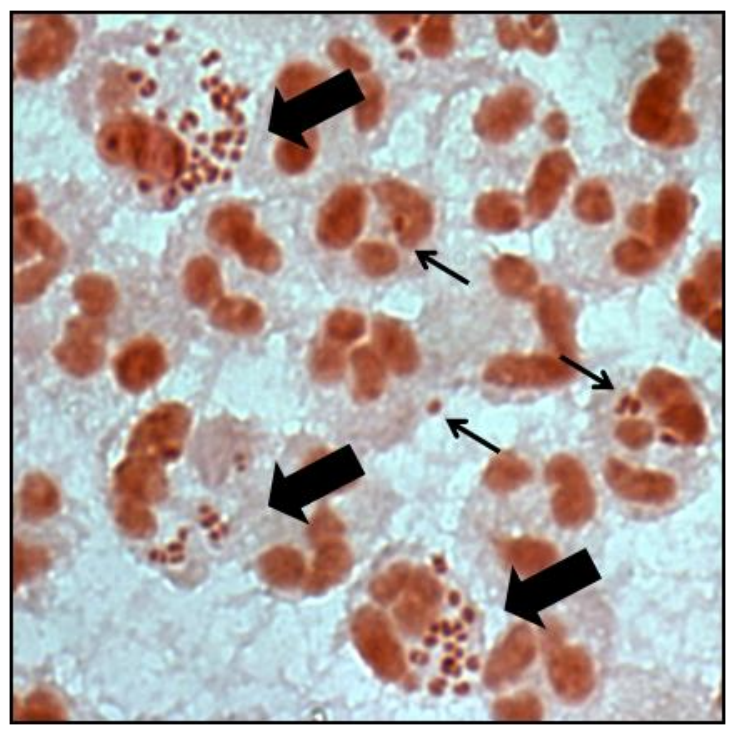

Figure 1: Gonorrhoeal exudates contain numerous PMNs with associated Gc.

Gram stain of the urethral exudate from a male with uncomplicated gonorrhea. Some PMNs associate with single diplococci (thin arrow), while others have multiple adherent and internalized Gc (thick arrow). Note that the majority of PMNs in the exudate are uninfected. 


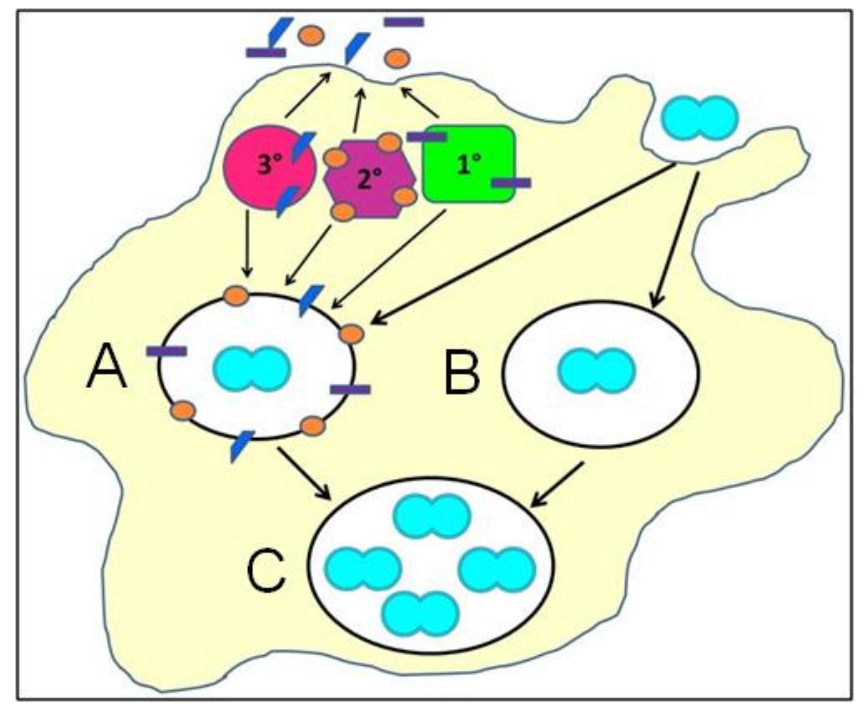

Figure 2: Cellular mechanisms of Gc survival after exposure to PMNs.

Gc (blue diplococcus) attaches to the surface of PMNs and is engulfed into a phagosome (white oval). PMNs possess three classes of granules $\left(1^{\circ}, 2^{\circ}\right.$, and $\left.3^{\circ}\right)$, each of which contains a unique subset of antimicrobial compounds. Granules fuse with the nascent phagosome or plasma membrane to deliver their contents to invading microorganisms. We propose two mechanisms that allow Gc to survive after exposure to PMNs. (For illustrative purposes, only intracellular Gc survival is depicted.) First, PMN granules release their contents at the plasma membrane or into phagosomes containing Gc (A). However, Gc virulence factors confer resistance to granules' antimicrobial compounds. Second, Gc prevents PMN granules from releasing their contents at the plasma membrane or into phagosomes, allowing the bacteria to avoid encountering PMN antimicrobial compounds (B). Either mechanism would enable a fraction of Gc to survive and replicate in the presence of PMNs (C). 


\begin{tabular}{|l|l|}
\hline Granule class & Granule components \\
\hline Primary/azurophilic & $\begin{array}{l}\text { Cathepsin G, BPI, Lysozyme, elastase, } \\
\text { myeloperoxidase (MPO), } \alpha \text {-defensins }\end{array}$ \\
\hline Secondary/specific & $\begin{array}{l}\text { Flavocytochrome } \mathbf{b}_{558}, \text { LL-37 (hCAP18), } \\
\text { lysozyme, gelatinase, lactoferrin, } \\
\text { CD11b/CD18 (CR3) }\end{array}$ \\
\hline Tertiary/gelatinase & $\begin{array}{l}\text { Flavocytochrome } \mathbf{b}_{558}, \text { lysozyme, } \\
\text { gelatinase, CD11b/CD18 (CR3) }\end{array}$ \\
\hline
\end{tabular}

Table 1: Antimicrobial components housed in PMN granules.

Proteins that have been shown to have or produce antimicrobial activity against Gc in vitro are bolded and italicized. Proteins to which Gc is resistant are indicated in red type. 


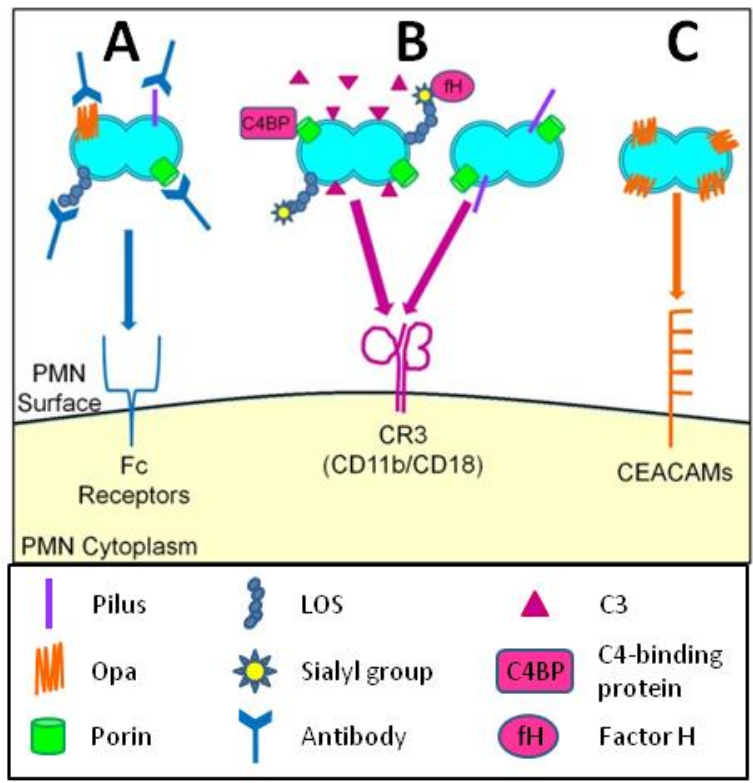

Figure 3: Opsonic and non-opsonic phagocytosis of Gc by PMNs.

(A) Antibodies that recognize Gc surface structures opsonize the bacteria and allow for phagocytosis via Fc receptors. The efficacy of immunoglobulin-mediated phagocytosis is questionable given the extensive phase and antigenic variation of Gc surface structures. (B) $\mathrm{Gc}$ binds factor $\mathrm{H}$ and $\mathrm{C} 4$ binding protein, resulting in opsonization of $\mathrm{Gc}$ with $\mathrm{C} 3$ and other complement components. Gc is then phagocytosed via the CR3 receptor. Gc pili and porin can cooperatively interact with $\mathrm{CR} 3$, which may mediate the non-opsonic phagocytosis of Gc by PMNs. (C) Selected Opa proteins bind to CEACAM family receptors expressed on PMNs, leading to non-opsonic phagocytosis of $\mathrm{Opa}^{+} \mathrm{Gc}$. 


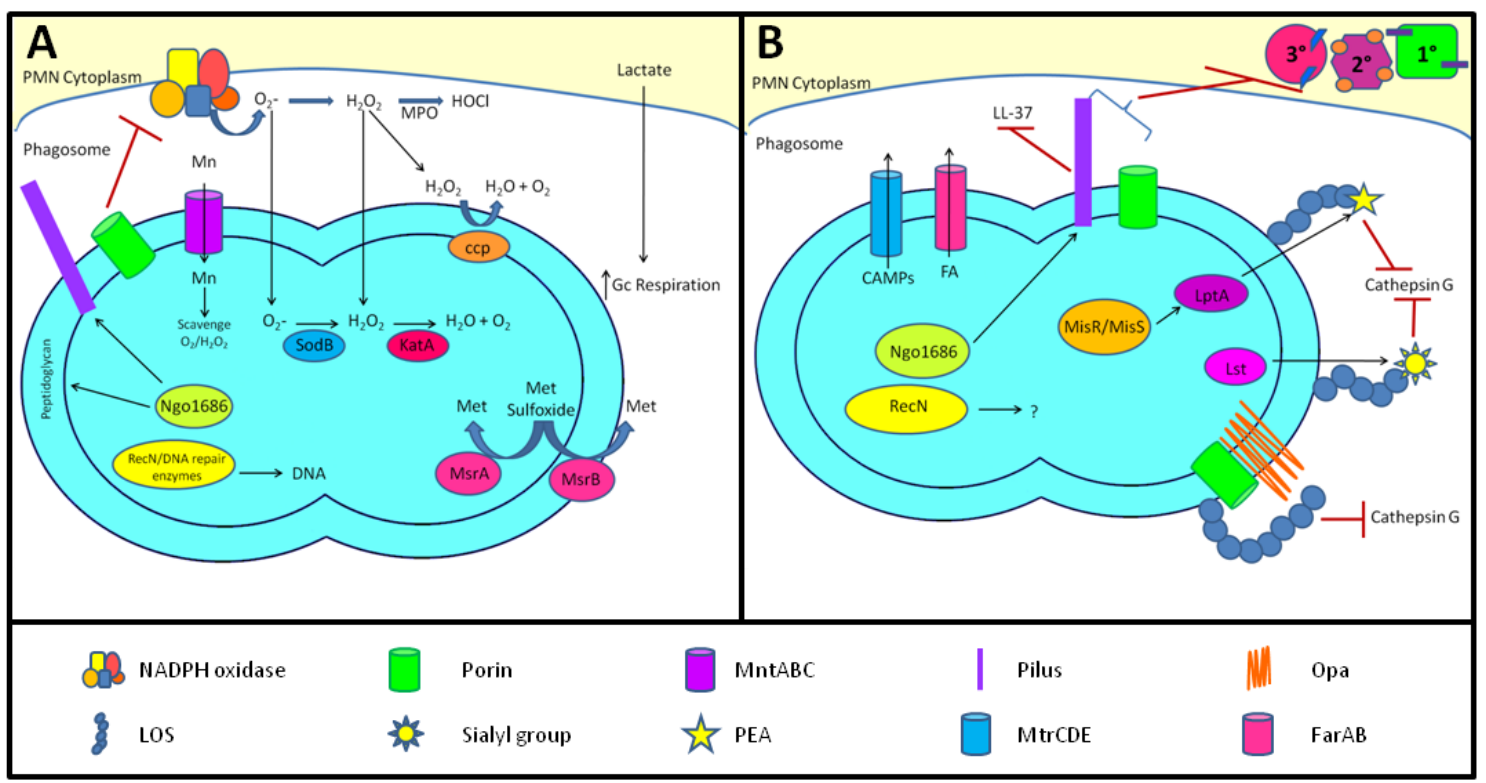

Figure 4: Mechanisms of Gc survival after exposure to antimicrobial compounds produced by PMNs.

(A) Resistance to oxidative damage. PMN NADPH oxidase generates superoxide $\left(\mathrm{O}_{2}^{-}\right)$ and hydrogen peroxide $\left(\mathrm{H}_{2} \mathrm{O}_{2}\right)$ from $\mathrm{O}_{2}$, which are converted to hypochlorous acid ( $\mathrm{HOCl}$ ) by myeloperoxidase (MPO). Gc prevents PMNs from generating ROS by lactatemediated increase in $\mathrm{Gc}_{2}$ consumption and suppression of NADPH oxidase activity by porin or as-yet unidentified factors. Gc scavenges ROS through the activities of MntABC, superoxide dismutase (SodB), catalase (KatA), and cytochrome $c$ peroxidase (Ccp). Gc can also repair damage due to ROS through DNA repair enzymes (RecN), protein reductases (MsrA/B), and other proteins (Ngo1686). (B) Resistance to PMN nonoxidative damage. Gc pili and/or porins prevent PMN granules from releasing nonoxidative antimicrobial components. LOS protects Gc outer membrane proteins such as Opa and porin from proteolysis by cathepsin G. Sialylation of LOS by Lst and PEA modification of LOS by LptA increases bacterial resistance to cathepsin $\mathrm{G}$ and other antimicrobials. The MisR/MisS two-component regulator increases expression of LptA and other gene products that confer resistance to PMN non-oxidative damage. Ngo1686 and $\mathrm{RecN}$ also protect Gc from PMN non-oxidative damage. The MtrCDE and FarAB efflux pumps export cationic antimicrobial peptides (CAMPs) and long-chain fatty acids (FA) from the Gc cytoplasm, respectively. In most cases, the contribution of these virulence factors to Gc survival after exposure to PMNs remains to be determined. 


\section{Chapter 2: Neisseria gonorrhoeae phagosomes delay fusion with primary granules to enhance bacterial survival inside human neutrophils}

This chapter is a modified version of the previously published article, "Neisseria gonorrheae phagosomes delay fusion with primary granules to enhance bacterial survival inside human neutrophils" [Cell Microbiol., 8:1323-40, 2013. doi: 10.1111/cmi.12117].

\subsection{Introduction}

Neisseria gonorrhoeae (the gonococcus or Gc) is an obligate human pathogen and the sole cause of the sexually transmitted disease gonorrhea, a major global health problem. The World Health Organization estimates there are 106 million cases of gonorrhea worldwide each year(World Health Organization, 2012). 330,000 cases are reported annually in the United States, although it is estimated that the actual number is at least twice as high (Workowski et al., 2010). Gonorrhea typically presents as acute urethritis in men and cervicitis in women as a consequence of close sexual contact with an infected individual. Infection can lead to serious morbidity and complications if left untreated, including pelvic inflammatory disease, sterility, arthritis-dermatitis syndrome, endocarditis, and meningitis (Wiesner et al., 1980). Gonorrhea can also be transmitted during childbirth, a leading cause of neonatal blindness. Due to the sustained prevalence of gonorrhea throughout the world (Workowski et al., 2010), Gc acquisition of resistance to multiple antibiotics (Ohneck et al., 2011), and the inability to date to generate a protective vaccine (Zhu et al., 2011), gonorrhea remains a significant public health issue. 
During acute gonorrheal disease, the infected mucosa releases chemokines that initiate a potent inflammatory response and recruit abundant PMNs to the site of infection (Ramsey et al., 1995, Hedges et al., 1998). PMNs possess oxygen-dependent and oxygen-independent antimicrobial components contained within three subsets of cytoplasmic granules, primary, secondary, and tertiary (Faurschou et al., 2003, Segal, 2005). In response to infection, PMNs first release tertiary (gelatinase) granules, which contain enzymes including gelatinase to degrade the extracellular matrix and allow for migration across the tissue, as well as gp $91^{\text {phox }}$ and $\mathrm{p} 22^{\text {phox }}$ subunits of the NADPH oxidase complex that generates reactive oxygen species. At the site of infection, PMNs release secondary and primary granules. Secondary (specific) granules contain complement receptor $3(\mathrm{CD} 11 \mathrm{~b} / \mathrm{CD} 18)$, the gp91 ${ }^{\text {phox }}$ and $\mathrm{p} 22^{\text {phox }}$ NADPH oxidase subunits, and oxygen-independent antimicrobial products such as lactoferrin and hCAP18, the precursor to the cathelicidin LL-37. Primary (azurophilic) granules contain the majority of PMN antimicrobial products, including myeloperoxidase (MPO) to generate hypochlorous acid, $\alpha$-defensin antimicrobial peptides, bactericidal-permeabilityincreasing protein (BPI), and the serine proteases cathepsin G, neutrophil elastase, and proteinase 3 . In contrast to phagosomes in macrophages and nonphagocytic cells, PMN phagosomes are not especially acidic and even undergo an initial period of alkalinization (Lee et al., 2003). Therefore, clearance of microbes in PMNs is primarily due to the coordinate release of PMN granules which enhances PMN phagocytosis, generates a potent oxidative burst, and releases antimicrobial peptides and proteases (Segal, 2005).

While numerous PMNs are present at the site of Gc infection, PMNs cannot resolve gonorrheal disease, and viable Gc can be cultured from the purulent exudates of 
gonorrhea patients (Wiesner et al., 1980). Moreover, in primary human PMNs infected in vitro with Gc, a significant percentage of internalized bacteria remain viable (Simons et al., 2005, Criss et al., 2009). These findings indicate that Gc has evolved mechanisms to resist PMN killing (Johnson et al., 2011). We and others have shown that Gc suppresses the ability of PMNs to produce ROS, and virulence factors that defend the bacteria against oxidative damage do not contribute to Gc survival in PMNs (Criss et al., 2008, Britigan et al., 1988, Lorenzen et al., 2000, Bjerknes et al., 1995, Seib et al., 2005, Wu et al., 2009). Thus Gc survival after exposure to PMNs is primarily due to the bacteria resisting oxygen-independent PMN antimicrobial activities. A variety of Gc gene products contribute to bacterial resistance to oxygen-independent antimicrobial peptides and proteins, resulting in modifications to Gc lipooligosaccharide and expression of efflux pumps (reviewed in (Johnson et al., 2011). While purified cathepsin G, BPI, and LL-37 have antigonococcal activity in vitro (Rest, 1979, Rock et al., 1988, Shafer et al., 1986a, Shafer et al., 1998, Casey et al., 1985), their effects on Gc viability have not been examined in the context of bacterial interactions with PMNs.

In this study we investigated cellular mechanisms that contribute to Gc intracellular survival in adherent, IL-8 treated, primary human PMNs. To our surprise, the majority of Gc phagosomes exhibited delayed fusion with PMN primary granules. In the absence of immediate fusion with primary granules, phagosomes were more likely to contain viable Gc. Increasing primary granule fusion with Gc phagosomes reduced the viability of the bacteria inside PMNs. We present a model in which surface characteristics of Gc drive bacteria into phagosomes that initially lack primary granule proteins, which have antigonococcal activity. The delay in primary granule fusion with 
Gc phagosomes supports the survival of Gc inside these normally antimicrobial cells, thereby contributing to the persistence of Gc in its obligate human host.

\subsection{Materials and Methods}

\subsubsection{Bacterial strains and growth conditions}

The Gc of strain FA1090 used in this study constitutively expresses the pilin variant 181-S2 due to mutation of the guanine quartet sequence upstream of pilE (Cahoon et al., 2009) and has in-frame deletions of the genes encoding the 4 "translucent" opacityassociated (Opa) proteins, Opas B, E, G, and K (Ball et al., 2013). Piliated, phenotypically Opa-negative Gc was routinely grown on Gonococcal Medium Base (Difco) plus Kellogg's supplements (Kellogg et al., 1963) for $20 \mathrm{~h}$ at $37^{\circ} \mathrm{C}$ in $5 \% \mathrm{CO}_{2}$. Gc was grown to exponential phase via successive rounds of bacterial growth in rich liquid medium, as described (Criss et al., 2008). Unless otherwise stated, viable, exponential phase Gc was used to infect primary human PMNs. To kill Gc prior to PMN exposure, Gc was either heat killed by incubation at $56^{\circ} \mathrm{C}$ for $20 \mathrm{~min}$, or fixed for $15 \mathrm{~min}$ with 4\% paraformaldehyde (PFA) (Electron Microscopy Sciences) in PBS. Gc was

labeled with $5 \mu \mathrm{g} \mathrm{ml}^{-1}$ carboxyfluorescein diacetate succinimidyl ester (CFSE) for $20 \mathrm{~min}$ at $37^{\circ} \mathrm{C}$. Gc was complement opsonized with $50 \%$ fresh normal human serum (in Morse's defined medium (MDM) (Morse et al., 1980)) for 20 min at $37^{\circ} \mathrm{C}$. Gc was IgG opsonized with a polyclonal rabbit anti-Gc antibody (Biosource) for $30 \mathrm{~min}$ at $37^{\circ} \mathrm{C}$. FA1090 iga::kan Gc, lacking IgA protease, was previously described (Criss et al., 2008). S. aureus ATCC 25923 and a $\triangle$ spa mutant generated in the Newman background (obtained from E. Skaar, Vanderbilt University) were grown on Luria-Bertani (LB) agar for $16 \mathrm{~h}$. S. aureus was inoculated into LB liquid broth and grown with rotation at $37^{\circ} \mathrm{C}$ 
for $20 \mathrm{~h}$. In immunofluorescence experiments, prior to infection, S. aureus was prelabeled with $10 \mu \mathrm{g} \mathrm{ml}^{-1}$ 4',6-diamidino-2-phenylindole (DAPI) (Sigma) for 20 minutes in MDM at room temperature in the dark. S. aureus was complement opsonized with $20 \%$ fresh normal human serum in MDM for 20 min at $37^{\circ} \mathrm{C}$.

\subsubsection{Zymosan}

Zymosan (MP Biomedicals) was opsonized in 50\% human serum in RPMI for 25 min at $37^{\circ} \mathrm{C}$.

\subsubsection{PMN isolation}

Venous blood was collected from healthy human subjects after receiving written informed consent, in accordance with a protocol approved by the University of Virginia Institutional Review Board for Health Sciences Research (IRB-HSR). PMNs were isolated from heparinized blood by dextran sedimentation followed by purification on a Ficoll-Hypaque gradient as previously described (Stohl et al., 2005). PMNs were

resuspended at $1 \times 10^{7}$ cells per $\mathrm{ml}$ in Dulbecco's PBS (without calcium and magnesium; Thermo Scientific) containing 0.1\% dextrose and kept on ice until use within $2 \mathrm{~h}$. Preparations routinely contained $>95 \%$ PMNs, assessed morphologically by phasecontrast microscopy.

\subsubsection{Human gonorrheal exudates}

PMN-rich urethral gonorrheal exudates were obtained from men attending the Virginia Department of Health (VDH) sexually transmitted disease clinic in Charlottesville, Virginia, after receiving written informed consent, in accordance with a protocol approved by the University of Virginia IRB-HSR and the VDH. Exudates from male subjects presenting with symptoms consistent with acute, uncomplicated gonorrhea were 
collected via urethral swab into $4 \%$ PFA in PBS and stored at $4{ }^{\circ} \mathrm{C}$ until use. Gram staining of the exudate prior to fixation and nucleic acid amplification tests confirmed the subjects were positive for gonorrhea and no other sexually transmitted infection.

\subsubsection{Adherent PMN assay}

Acid washed $12 \mathrm{~mm}$ glass coverslips in 24 -well plates were coated for $1 \mathrm{~h}$ at $37^{\circ} \mathrm{C}$ with $50 \%$ pooled human serum (Sigma) in PBS and washed in PBS prior to PMN addition. PMNs were diluted into RPMI (Mediatech) containing 10\% fetal bovine serum (Thermo Scientific) and 10nM IL-8 (R\&D Systems). $10^{6}$ PMNs were added to each coverslip and incubated for $1 \mathrm{~h}$ at $37^{\circ} \mathrm{C}, 5 \% \mathrm{CO}_{2}$ to promote $\mathrm{PMN}$ attachment. PMNs were infected with $S$. aureus, viable exponential-phase Gc, or exposed to heat killed or PFA-killed Gc at a multiplicity of infection of 1-5 bacterial colony forming units (CFU) per PMN as described in (Criss et al., 2009). PMNs were exposed to opsonized zymosan for 25 min at $37^{\circ} \mathrm{C}$. For experiments extending Gc infection to $6 \mathrm{~h}$, after $1 \mathrm{~h}$ media was replaced with Hanks' balanced salt solution with $\mathrm{Ca}^{2+}$ and $\mathrm{Mg}^{2+}$ and supplemented with $0.15 \%$ dextrose and $1 \%$ BSA.

\subsubsection{Gc and S. aureus Coinfection:}

PMNs were infected with Gc as described. After 30 min PMNs were infected with nonopsonized DAPI labeled S. aureus at a multiplicity of infection of 1-5 CFU per PMN. S. aureus was spun into contact with PMNs at $400 \mathrm{xg}$ for $4 \mathrm{~min}$ at $12^{\circ} \mathrm{C}$. Cells were incubated $30 \mathrm{~min}$ at $37^{\circ} \mathrm{C}$. PMNs infected with Gc and S. aureus were fixed with $4 \%$ PFA in PBS for 15 min. 


\subsubsection{Immunofluorescence}

2.2.7.1 PMNs infected in vitro with Gc: PMNs infected for 1, 2, 4, and $6 \mathrm{~h}$ with Gc or $S$. aureus were fixed with 4\% PFA in PBS for 15 min. External and internal Gc were distinguished using a polyclonal rabbit anti-Gc antibody before and after PMN permeabilization as previously described in (Criss et al., 2009). For experiments examining the secondary granule marker lactoferrin, Gc was labeled prior to infection with CFSE to stain the total Gc population, and external Gc were detected using the polyclonal rabbit anti-Gc antibody as described above. External S. aureus were recognized using a polyclonal biotinylated S. aureus antibody (MyBiosource) followed by exposure to streptavidin coupled to Alexa Fluor 555 (Life Technologies), prior to PMN permeabilization. Cells were then permeabilized using a 1:1 ratio of acetone and methanol. Post permeabilization, PMN secondary granules were recognized using monoclonal antibodies against $\mathrm{p} 22^{\text {phox }}(44.1)$ and gp91 ${ }^{\text {phox }}$ (54.1) (both Santa Cruz Biotechnology), or a polyclonal rabbit anti-lactoferrin antibody (MP Biomedicals). PMN primary granules were recognized using monoclonal antibodies against CD63 (H5C6-c) (Developmental Studies Hybridoma Bank and Ancell) or neutrophil elastase (AHN-10) (Millipore) followed by Alexa Fluor-coupled goat anti-rabbit or goat anti-mouse antibodies (Life Technologies). Coverslips were mounted using Flouromount G (Southern Biotech) with $2.5 \mathrm{mg} \mathrm{ml}^{-1}$ propyl gallate (Acros Organics).

2.2.7.2 Human gonorrheal exudates: Gonorrheal exudates were pelleted at $100 \mathrm{x} g$ for 12 min. Cells were resuspended in PBS containing 10\% normal goat serum (Life Technologies) and $0.2 \%$ saponin, then incubated for $1 \mathrm{~h}$ with Alexa Fluor 555-coupled mouse anti-CD63 antibody to label primary granules and $10 \mu \mathrm{g} \mathrm{ml}^{-1}$ DAPI to label total 
Gc. The anti-CD63 antibody was coupled to Alexa Fluor 555 using a Molecular Probes monoclonal antibody labeling kit (Life Technologies), following the manufacturer's instructions. Cells were washed in PBS and mounted on slides for imaging.

\subsubsection{Bacterial Viability Dyes}

Protocols describing the detailed use of fluorescent dyes to detect the viability of bacteria inside and outside of host cells are available in Appendix A (Johnson et al., 2013a).

\subsubsection{Life Technologies Baclight Viability Dyes: PMNs were infected with Gc for $1 \mathrm{~h}$.}

Samples were washed and incubated for $10 \mathrm{~min}$ at $\mathrm{RT}$ in $\mathrm{MOPS} / \mathrm{MgCl}_{2}$ containing $5 \mu \mathrm{g}$ $\mathrm{ml}^{-1}$ Alexa Fluor 647-coupled soybean agglutinin (Life Technologies), which recognizes extracellular Gc. PMNs were then incubated with the $30 \mu \mathrm{M}$ propidium iodide and $5 \mu \mathrm{M}$ SYTO9 (Baclight Viability Dyes Invitrogen) for $15 \mathrm{~min}$ in $\mathrm{MOPS} / \mathrm{MgCl}_{2}$ containing $0.2 \%$ saponin. Gc were counted as viable if labeled with SYTO9 and non-viable if labeled with propidium iodide.

\subsubsection{Sytox Green in combination with DAPI: A modification of the Baclight-based} bacterial viability assay was developed since propidium iodide emits in the red and ultraviolet channels of the fluorescence microscope, and detection of external vs. internal bacteria, viable bacteria, nonviable bacteria, and CD63 requires four-color fluorescence microscopy. Gc were prelabeled for 20 min with $10 \mu \mathrm{g} \mathrm{ml}^{-1}$ DAPI in MDM. After $1 \mathrm{~h}$ of PMN infection, samples were incubated with $0.4 \mu \mathrm{M}$ Sytox Green (Life Technologies) in 0.1 MOPS pH 7.2, $1 \mathrm{mM} \mathrm{MgCl} 2\left(\mathrm{MOPS} / \mathrm{MgCl}_{2}\right)$ for $5 \mathrm{~min}$. Gc were counted as viable if labeled only with DAPI and non-viable if labeled with DAPI and Sytox Green. 
2.2.9 Immunofluorescence for CD63 in combination with bacterial viability dyes PMNs were infected with DAPI-labeled Gc. After $1 \mathrm{~h}$, samples were washed and incubated for $10 \mathrm{~min}$ at $\mathrm{RT}$ in $\mathrm{MOPS} / \mathrm{MgCl}_{2}$ containing $5 \mu \mathrm{g} \mathrm{ml}^{-1}$ Alexa Fluor 647coupled soybean agglutinin (Life Technologies), which recognizes extracellular Gc. PMNs were then incubated with Alexa Fluor 555-coupled mouse anti-CD63 antibody for 20 min in MOPS/ $\mathrm{MgCl}_{2}$ containing $0.2 \%$ saponin. PMNs were then exposed to $0.4 \mu \mathrm{M}$ Sytox Green in MOPS/ $\mathrm{MgCl}_{2}$ containing $0.2 \%$ saponin for 5 min to stain nonviable Gc.

\subsubsection{Immuno-TEM}

PMNs infected for $1 \mathrm{~h}$ with Gc or $\triangle$ spa S. aureus were fixed with 2\% PFA, $0.25 \%$ glutaraldehyde in phosphate buffer $\mathrm{pH} 7.2$ for $45 \mathrm{~min}$. PMNs were then incubated in $1 \%$ PFA in phosphate buffer and shipped to the University of Iowa Central Microscopy Research Facility. Cells were embedded in LR White and 60nm thin sections placed on Formvar carbon coated grids. Sections were stained with polyclonal antibodies against the lactoferrin (MP Biomedicals) or myeloperoxidase (Dako), followed by 10nm gold particle-labeled secondary goat anti-rabbit antibody. Sections were examined with a JEOL 1230 transmission electron microscope at an accelerating voltage of $120 \mathrm{kV}$.

\subsubsection{PMN Treatments}

2.2.11.1 Lysophosphatidylcholine ( $L P C)$ : PMNs were infected with Gc as described. After 45 min, fresh RPMI +10\% FBS or media containing 30 $\mu$ M LPC (18:0) (SigmaAldrich; reconstituted in PBS) was added to each well and incubated for $15 \mathrm{~min}$ at $37^{\circ} \mathrm{C}$. PMNs were washed with fresh media and fixed for immunofluorescence or incubated an additional $30 \mathrm{~min}$ in fresh media and processed using Baclight viability dyes as described above. Immunofluorescence was conducted as described to discriminate extracellular vs. intracellular Gc and to detect neutrophil elastase. 
2.2.11.2 Protease Inhibitors: PMNs were incubated with $1 \mathrm{X}$ Protease Inhibitor Cocktail Set V (Calbiochem) containing AEBSF, aprotinin, E-64, and leupeptin, during PMN attachment to serum coated coverslips. Protease inhibitors were present for the entire experiment. PMNs were infected with Gc for 90 min. After 45 min, protease inhibitortreated PMNs were exposed to LPC. Gc viability was assessed using Baclight viability dyes.

2.2.11.3 Ionomycin: PMNs were infected with Gc as described. After $30 \mathrm{~min}$, fresh RPMI $+10 \%$ FBS or media containing $1 \mu \mathrm{M}$ ionomycin was added to each well and incubated for $15 \mathrm{~min}$ at $37^{\circ} \mathrm{C}$. PMNs were washed with fresh media and fixed for immunofluorescence.

2.2.11.4 Determination of serine protease activites: The activity of cathepsin G, neutrophil elastase, and proteinase 3 were assessed using substrates specific to these serine proteases: cathepsin G, 0.1mM Suc-Ala-Ala-Pro-Phe-pNA (Elastin Products Company) in $100 \mathrm{mM}$ HEPES $500 \mathrm{mM} \mathrm{NaCl} \mathrm{pH}$ 7.5; neutrophil elastase, $0.85 \mathrm{mM}$ MeOSuc-Ala-Ala-Pro-Val-pNA (Calbiochem) in 100 mM HEPES 500 mM NaCl pH 7.5 ; proteinase 3, $0.25 \mathrm{mM}$ Boc-Ala-Ala-Nva-SBzL (Elastin Products Company) in $\mathrm{H} 2 \mathrm{O}$ with 0.1mM 5,5'- Dithiobis (2-nitro-benzoic acid) (Sigma-Aldrich). PMNs were incubated in the presence or absence of 1X Protease Inhibitor Cocktail Set V. After 1 hour the media was removed. The appropriate media for each substrate containing $0.01 \%$ Triton X-100 was added to each well and PMNs were scrapped off of coverslips. The media containing PMNs was incubated for 5 min on ice. Debris was removed by spinning 0.8 x g for 8 min. An equal volume of lysed PMNs and substrate for indicated serine 
protease was added in triplicate to wells of a 96 well plate. The plate was incubated in the dark for $45 \mathrm{~min}$ at $37^{\circ} \mathrm{C}$. The $\mathrm{OD}_{405}$ of each reaction was read on a Perkin Elmer Victor 31420 microplate reader.

\subsubsection{Image acquisition, processing, and quantification}

Images were acquired on a Nikon Eclipse E800 with Hamamatsu Orca-ER digital camera using Openlab software and processed in Adobe Photoshop CS5. For consistency, all images depicting total bacteria were false colored blue, including bacteria labeled with CFSE. Images of human gonorrheal exudates were acquired on a Zeiss LSM510 confocal laser scanning microscope with a 63x, 1.4 numerical aperture objective. Images were acquired with LSM510 operating software and processed with LSM Image Browser (Zeiss) and/or Adobe Photoshop CS5 (Adobe).

2.2.12.1 Immunofluorescence: 50-200 intracellular bacteria were analyzed for enrichment of PMN granule proteins. Bacterial phagosomes were classified as positive for PMN granule proteins if antibody staining surrounded $\geq 50 \%$ of the bacterial circumference.

2.2.12.2 Bacterial viability dyes: Images were acquired within $30 \mathrm{~min}$ of mounting coverslips and a minimum of 100 bacteria per experiment were analyzed for viability.

\subsubsection{Immunofluorescence for CD63 in combination with bacterial viability dyes:}

Images were acquired within 30 min of mounting coverslips. A minimum of 50 internalized bacteria per experiment were analyzed for viability and enrichment of the PMN primary granule protein CD63 at the Gc phagosome. Phagosomes were classified 
as positive for CD63 according to guidelines described for immunofluorescence experiments.

2.2.12.4 Immuno-TEM: In order to determine if secondary and primary granules fuse with S. aureus and Gc phagosomes, samples were stained using anti-lactoferrin or antiMPO antibodies. Bacteria phagosomes were examined for the presence of lactoferrin or MPO within the phagosomes and lactoferrin or MPO positive granules surrounding the phagosome. 200 bacteria from samples stained with the anti-MPO antibody were analyzed for the presence of an electron dense MPO positive ring surrounding the bacteria and the electron density of each bacterium was noted to assess bacterial viability.

2.2.12.5 Human gonorrheal exudate: A minimum of 100 internalized bacteria were analyzed for enrichment of the PMN primary granule protein CD63 at the Gc phagosome. Phagosomes were classified as positive for CD63 according to guidelines described for immunofluorescence with ex vivo-infected primary human PMNs.

\subsubsection{Statistics}

All values are expressed as a mean \pm standard error of the mean for three replicate experiments (unless otherwise noted), performed on different days with different donors' PMNs for each assay. Significance was determined for each assay using a Student's twotailed t-test. A $p$ value of less than 0.05 was considered statistically significant.

\subsection{Results}

\subsubsection{Gc phagosomes in primary human PMNs undergo limited fusion with primary granules}

To assess the maturity of PMN phagosomes containing Gc, adherent, IL-8 primed, primary human PMNs were infected with the Gc strain FA1090, and the 
enrichment and fusion of granule subsets with bacterial phagosomes was observed by immunofluorescence and immuno-transmission electron microscopy (TEM). Piliated, Opa-negative Gc were used to remain consistent with previous studies (Criss et al., 2009, Stohl et al., 2005). Maturation of Gc phagosomes was compared to S. aureus-containing phagosomes, which fuse with all subsets of granules (Voyich et al., 2005). For immunofluorescence, bacterial phagosomes were considered enriched for a PMN granule subset if antibody reactivity against a protein found in that granule subset surrounded $\geq 50 \%$ of the bacterial circumference.

We initially compared $S$. aureus and Gc phagosomes for the presence of proteins found in secondary and tertiary granules, which are mobilized early in PMN activation (Faurschou et al., 2003). These granule subsets were detected with antibodies against the membrane proteins gp $91^{\text {phox }}$ and $\mathrm{p} 22^{\text {phox }}$. As expected, the majority of $S$. aureus phagosomes were enriched for gp $91^{\text {phox }}$ and $\mathrm{p} 22^{\text {phox }}$ after $1 \mathrm{~h}$ infection (Figure $5 \mathrm{~A}$ and $\mathrm{B}$ ). We observed similar enrichment of lactoferrin, a protein found within secondary granules, in S. aureus phagosomes (Figure 5C and D). After $1 \mathrm{~h}$ infection, Gc phagosomes were enriched for secondary and tertiary granule proteins to the same extent as $S$. aureus phagosomes (Figures 5B and 5D). We then conducted immuno-TEM using an anti-lactoferrin antibody to assess whether or not secondary granules fuse with $S$. aureus and Gc phagosomes. All bacteria were found to reside in membrane-bound compartments inside PMNs. Both S. aureus (Figure 5E and 5F) and Gc (Figure 5G and 5H) infected PMNs exhibited lactoferrin staining within phagosomes, as well as lactoferrin-positive granules surrounding the phagosomes. From these data we conclude 
that secondary granules, and presumably tertiary granules, fuse with Gc phagosomes in PMNs.

As the final step in PMN activation, PMNs mobilize primary granules, which contain the majority of PMN antimicrobial peptides and proteases (Faurschou et al., 2003). We examined enrichment of primary granules to $S$. aureus and Gc phagosomes by immunofluorescence against the membrane protein CD63 and the content protein neutrophil elastase. As anticipated, S. aureus phagosomes were highly enriched for primary granule proteins after $1 \mathrm{~h}$ infection, where solid rings of CD63 or neutrophil elastase staining were seen surrounding intracellular bacteria (Figure 6A and 6C). In contrast, there was a significant decrease in the percent of Gc phagosomes enriched for CD63 or neutrophil elastase after $1 \mathrm{~h}$ infection (Figure 6B and 6D). While punctate CD63 and neutrophil elastase staining was detected in the vicinity of some Gc phagosomes (Figure 6A and 6C), this staining pattern did not meet the criteria for phagosomal granule enrichment. Immuno-TEM against the primary granule protein MPO confirmed that S. aureus phagosomes fused with primary granules, with MPOpositive granules also seen surrounding and docking to the phagosomal membrane (Figures 6E and 6F). Inside S. aureus phagosomes, MPO reactivity appeared to form a ring around the bacteria as it decorated the bacterial surface. Similar to observations noted for S. aureus, some Gc phagosomes exhibited fusion with MPO positive granules (Figure 6G). However, Gc phagosomes that lacked the electron dense, MPO positive ring were also observed, even though MPO-positive primary granules were in their vicinity (Figure 6H). The immunofluorescence and immuno-TEM results indicate that most Gc 
phagosomes do not fuse with primary granules, such that primary granule contents, including MPO and neutrophil elastase, are likely not delivered to phagosomes.

In order to determine if Gc phagosomes avoid fusion with primary granules in PMNs in vivo, we examined PMNs in human gonorrheal exudates for enrichment of CD63 around Gc. Similar to observations in ex vivo-infected PMNs, an average of $60 \%$ of Gc associated with exudatous PMNs were not enriched for CD63, although occasional examples of Gc surrounded by CD63 staining were observed (Figure 7A). This observation supports our findings using adherent, IL-8 primed, primary human PMNs.

To address whether the absence of primary granule enrichment on Gc phagosomes represented a block or a delay in phagosome-granule fusion, maturation of Gc phagosomes in primary human PMNs was assessed over $6 \mathrm{~h}$. The presence of CD63 on Gc phagosomes steadily increased over time, from $47 \%$ after $1 \mathrm{~h}$ to $70 \%$ after $6 \mathrm{~h}$; similar results were obtained using neutrophil elastase to mark primary granules (Figure 8). We conclude that Gc delays primary granule fusion with the phagosome, thereby initially avoiding exposure to primary granule components inside PMNs.

In order to determine if the observed delay in primary granule fusion with Gc phagosomes is a result of Gc globally impacting PMN primary granule fusion, PMNs were coinfected with $S$. aureus and Gc. Primary granule fusion to $S$. aureus and Gc phagosomes in coinfected PMNs was compared to PMNs infected with S. aureus only or Gc only. There was no significant difference between the percent of S. aureus phagosomes positive for neutrophil elastase in coinfected PMNs or PMNs infected with S. aureus (Figure 9). These data indicate that Gc infection does not alter PMN primary 
granule fusion to $S$. aureus phagosomes. However, the percent of Gc phagosomes positive for neutrophil elastase in coinfected PMNs was significantly increased compared to PMNs infected with Gc alone (Figure 9), suggesting $S$. aureus can serve as a stimulus to increase Gc phagosome fusion with primary granules.

Taken together, these results reveal that the Gc inside human PMNs reside in two subsets of phagosomes. Early in infection, the majority of Gc phagosomes remain immature, since they do not fuse with primary granules but can fuse with other granule subsets. The remaining Gc reside in phagolysosomes that have fused with all classes of PMN granules. As infection proceeds, Gc phagosomes undergo fusion with primary granules, reaching a maximal percent maturity after $6 \mathrm{~h}$. The observed delay in primary granule fusion to Gc phagosomes is not due to Gc globally altering PMN primary granule fusion, but rather primary granule fusion with Gc phagosomes is determined on an individual phagosome basis.

\subsubsection{The delay in primary granule fusion with Gc phagosomes does not require active bacterial processes and can be overcome by IgG opsonization}

We envisioned two possibilities as to how Gc delays primary granule fusion with

its phagosomes in PMNs: live Gc releases factors that actively prevent early granule mobilization, or surface components on Gc influence phagosome biogenesis and granule mobilization. To test between these possibilities, PMNs were allowed to internalize nonviable, paraformaldehyde (PFA)-fixed Gc, and the composition of the Gc phagosomes was assessed by immunofluorescence microscopy. PMNs exposed to nonviable Gc exhibited reduced enrichment of neutrophil elastase and CD63 at their phagosomes, at levels statistically indistinguishable from PMNs infected with viable Gc 
(Figure 10). Similar results were obtained using heat-killed bacteria (data not shown). We conclude that active Gc processes are not required to prevent the initial fusion of primary granules with the Gc phagosome, suggesting instead that bacterial surface composition influences phagosome-granule fusion and delays phagosomal maturation.

In phagocytes, the route by which a particle enters the cell influences phagosomal maturity, and by extension, the phagosome's ability to kill microbes (Stuart et al., 2005, Lee et al., 2003). Directing bacterial pathogens such as Mycobacterium tuberculosis and Francisella tularensis to opsonophagocytic pathways reduces the survival of these bacteria inside PMNs and macrophages, respectively (Cougoule et al., 2002, Geier et al., 2011). We hypothesized that driving Gc uptake by PMNs via complement or Fc receptors would modulate phagosome maturation. To test this hypothesis, PMNs were infected with Gc that were opsonized with normal fresh human serum from individuals with no history of gonorrhea (serum opsonization), with anti-gonococcal rabbit IgG, or left unopsonized, and phagosome maturity was assessed by immunofluorescence microscopy. Gc opsonization with fresh human serum did not alter primary granule fusion with Gc phagosomes compared to non-opsonized Gc. However, IgG opsonization of Gc significantly increased primary granule fusion with Gc phagosomes compared to nonopsonized or complement-opsonized Gc (Figure 11). Similarly, we observed that opsonization of Gc with incremental concentrations of $\mathrm{IgG}$ resulted in increased primary granule fusion to Gc phagosomes (Figure 12). These results indicate that the surface composition of Gc, including its opsonization state, directs interactions with PMN receptors to influence phagosome-granule fusion and consequent phagosome maturation. However, the antibody used to opsonize Gc in these experiments is a polyclonal anti-Gc 
antibody, therefore, it is possible that the antibody is binding to a surface structure necessary for preventing primary granule fusion with the Gc phagosome.

\subsubsection{Gc phagosomes devoid of PMN primary granule proteins contain viable Gc}

During ex vivo infection of primary human PMNs, up to $50 \%$ of Gc inside PMNs

remain viable (Criss et al., 2009, Simons et al., 2005). As primary granules contain PMN components with known antigonococcal activity (Rest, 1979, Rock et al., 1988, Shafer et al., 1986b, Shafer et al., 1998, Casey et al., 1985), we hypothesized that primary granule fusion with Gc phagosomes reduces the viability of intracellular Gc, and the fraction of Gc that survive inside PMNs represent those bacteria residing in phagosomes that exclude primary granules. In preliminary support for this hypothesis, $86 \%$ of the phagosomes exhibiting rings of MPO staining by immuno-TEM, indicative of primary granule-phagosome fusion, contained electron-lucent, degraded bacteria (Figure 6G).

To directly examine the relationship between phagosome maturation and bacterial viability, we combined dyes that reveal viability of individual bacteria with the detection of primary granule proteins. We modified our previously published bacterial viability assay, which used propidium iodide permeability as a surrogate for bacterial death (Criss et al., 2009). Here, nonviable Gc are permeant to Sytox Green, while the total Gc population is counterstained with DAPI. We verified that the Sytox Green/DAPI combination was able to discriminate live and dead Gc inside and attached to PMNs as effectively as the propidium iodide/SYTO9 system (Figure 13). Detection of primary granule proteins was complicated by the fact that the bacterial viability dyes are incompatible with aldehyde fixation. However, a fluorescently coupled anti-CD63 antibody showed immunoreactivity with PMN granules and zymosan-containing 
phagolysosomes (Munafo et al., 2007) that colocalized with the pattern of CD63 staining in aldehyde-fixed cells (Figure 14).

To test the hypothesis that phagosomes lacking fusion with primary granules are more likely to contain viable Gc, PMNs were infected with DAPI-labeled Gc for $1 \mathrm{~h}$. PMNs were saponin-permeabilized without aldehyde fixation, then exposed to Sytox Green and fluorescently coupled anti-CD63. 25\% of intracellular, Sytox Green-negative, viable Gc showed enrichment of CD63. In contrast, $60 \%$ of intracellular, nonviable, Sytox Green-positive Gc were surrounded by CD63 immunoreactivity, a significant increase (Figure 15). Thus Gc viability inside PMNs correlates with bacterial residence in immature, primary granule-negative phagosomes.

The results presented thus far show that Gc have two fates within primary human PMNs. Most bacteria reside in phagosomes that undergo delayed primary granule fusion, while the remainder are found in phagosomes that fuse with primary granules and rapidly mature into phagolysosomes. This delay in phagosome maturation does not depend upon secretion or other active bacterial processes. However, when PMNs are infected with viable Gc, the fraction of bacteria that are killed within PMNs are more likely to be the ones residing in phagolysosomes, indicating that bacterial death inside PMNs occurs after phagosomes fuse with primary granules. We conclude that primary granule components have antigonococcal activity inside PMN phagosomes, and one mechanism by which Gc survives inside PMNs is by avoiding these components through a delay in phagosome maturation. 
2.3.4 Increasing primary granule fusion with Gc phagosomes reduces internal Gc viability

Given the correlation between Gc viability and residence in primary granulenegative phagosomes, we took two approaches to examine how manipulating phagosomal composition affected Gc viability inside PMNs. First, Gc was opsonized with IgG, in order to drive Fc $\gamma$ receptor-mediated uptake of the bacteria into phagolysosomes (Lee et al., 2003, Stuart et al., 2005) (see Figure 11). PMNs were infected with non-opsonized Gc or Gc opsonized with rabbit anti-Gc IgG, and bacterial viability inside PMNs was assessed using propidium iodide to detect bacteria with compromised membranes and SYTO9 for intact bacteria. IgG opsonization significantly decreased the viability of intracellular Gc, but did not affect the viability of adherent bacteria (Figure 16). Together with the results presented in Figure 11, these findings show that antibody opsonization directs Gc to a phagosome that undergoes early fusion with primary granules, which consequently decreases Gc intracellular survival.

As a second approach to modulating phagosome maturation, primary granule fusion with phagosomes was ectopically induced with lysophosphatidylcholine (LPC) treatment (Hong et al., 2010). Previously, it has been noted that increased primary granule fusion with PMN phagosomes due to LPC treatment is accompanied by calcium flux (Silliman et al., 2003). We observed LPC treatment (Figure 17A-B) and treatment with the calcium ionophore, ionomycin (Figure 18) significantly increased primary granule fusion with PMN phagosomes containing non-opsonized Gc (Figure 17A-B). As observed for IgG-opsonized Gc, intracellular Gc survival was significantly reduced in LPC-treated PMNs compared to the untreated control (Figure 17C-E). LPC treatment had 
no significant effect on the viability of Gc that were adherent to PMNs (Figure 17E) or were kept in media without PMNs (data not shown). From these results, we conclude that primary granules contain antimicrobial components that are capable of killing Gc inside PMN phagosomes, and driving primary granule fusion with Gc phagosomes by opsonization or LPC treatment decreases intracellular viability of the bacteria.

\subsubsection{Protease activity contributes to the anti-Gc activity of phagosomes enriched with primary granules}

Primary granules contain the majority of oxygen-independent PMN antimicrobial components (Faurschou et al., 2003). Of these components, Gc is resistant to $\alpha$-defensins (Qu et al., 1996) and suppresses the PMN oxidative burst, which provides the substrate $\mathrm{H}_{2} \mathrm{O}_{2}$ for MPO activity (Criss et al., 2008, Britigan et al., 1988, Lorenzen et al., 2000, Bjerknes et al., 1995, Seib et al., 2005, Wu et al., 2009). However, primary granule proteases, specifically the serine protease cathepsin $\mathrm{G}$, display antigonococcal activity in vitro (Rest, 1979, Rock et al., 1988, Shafer et al., 1986a, Shafer et al., 1998, Casey et al., 1985). Cathepsin G can enzymatically cleave Gc outer-membrane proteins (Rest et al., 1981, Shafer et al., 1987) and also has protease-independent antibacterial activity (Bangalore et al., 1990, Shafer et al., 1986b). The role of these primary granule components in killing Gc has not been examined in the context of PMN infection. To test whether PMN serine proteases contribute to the antigonococcal activities of mature PMN phagolysosomes, Gc viability was measured in PMNs treated with a protease inhibitor cocktail prior to infection (see Experimental procedures), in the presence or absence of LPC. To confirm treatment of PMNs with the protease inhibitor cocktail inhibited serine protease activity the activity of cathepsin G, neutrophil elastase, and proteinase 3 were 
assessed using substrates specific to these proteases (Figure 19F).In the absence of LPC treatment, protease inhibitor treatment significantly increased Gc viability inside PMNs compared to untreated PMNs (Figures 19A,C, quantified in E). Moreover, protease inhibitor exposure rescued the survival of Gc inside LPC-treated PMNs to levels seen in protease inhibitor-exposed PMNs not given LPC (Figures 19B,D,E). Protease inhibitor treatment did not affect the fusion of primary granules with PMN phagosomes (Figure 20). These data show for the first time that proteases are a major contributor to the antigonococcal activity associated with some PMN phagosomes. We conclude that by initially avoiding fusion with primary granules, the Gc phagosomes in PMNs exhibit reduced protease activity, which promotes Gc survival inside PMNs.

\subsection{Discussion}

Although there is evidence for Gc survival inside PMNs during acute gonorrhea, the mechanisms that allow the bacteria to avoid killing by PMNs have remained elusive. In this work we show for the first time that most Gc inside PMNs delay phagosome fusion with primary granules. These immature phagosomes are more likely to contain viable Gc, since primary granule proteases and other components have antigonococcal activity. Our findings reveal a novel approach Gc has evolved in order to evade PMN antimicrobial activities, thereby enabling its survival in its obligate human hosts.

We identified two compartments containing Gc in primary human PMNs. Most phagosomes fuse with secondary and/or tertiary granules but not primary granules, while others fuse with all granule subsets. Primary granules contain a variety of antimicrobial components including neutrophil elastase, cathepsin G, proteinase 3, BPI, and $\alpha$ defensins (Faurschou et al., 2003). Previously, purified BPI and cathepsin G have been shown to 
have antigonococcal activity in vitro (Casey et al., 1985, Shafer et al., 1986a). Our lab has demonstrated that the strain of Gc used in this manuscript, FA1090 is susceptible in vitro to BPI and $\alpha$-defensins; however, we have observed it is resistant to neutrophil elastase and cathepsin G (data not shown). Despite FA1090 being resistant in vitro to neutrophil elastase and cathepsin $\mathrm{G}$ it is still possible that in the context of the phagosome, these proteases in combination with the additional antimicrobial components or the additional antimicrobial components alone have antigonococcal activity. Thus we reasoned that delaying primary granule fusion with the Gc phagosome would confer a survival advantage on Gc found in PMNs. In support of this reasoning, nonviable Gc were more likely to be found in primary granule-positive phagosomes than viable bacteria. Moreover, ectopically increasing primary granule fusion with phagosomes by LPC treatment or antibody opsonization of Gc reduced intracellular bacterial viability. These observations strongly suggest that the components located within PMN primary granules are capable of killing Gc in the context of the PMN phagosome, which has never before been shown.

Our results point to serine proteases as important contributors to the antigonococcal activity of mature PMN phagosomes. The serine proteases of PMNs include neutrophil elastase, proteinase 3, cathepsin G, and NSP4 (Faurschou et al., 2003, Perera et al., 2012). We envision two possibilities to explain the contribution of these proteases to intraphagosomal Gc killing. First, the proteases act directly on Gc. In support of this possibility, in vitro studies have shown that cathepsin $\mathrm{G}$ degrades porin and Opa proteins in the Gc outer membrane (Rest et al., 1981, Shafer et al., 1987). Digestion of outer-membrane proteins could allow the proteases access to other potential 
targets inside Gc, although to date these are not known. It could also render the bacteria more sensitive to killing by non-proteolytic components of primary granules. These include BPI and cathepsin G itself, which contains two protease-independent peptide sequences with broad-spectrum antimicrobial activity in vitro (Bangalore et al., 1990). Second, primary granule serine proteases activate components with antigonococcal activity that are found in other PMN granule subsets. Secondary granules contain hCAP18, the precursor of the antimicrobial peptide LL-37 (Faurschou et al., 2003), which has antigonococcal activity in vitro (Shafer et al., 1998) and we have confirmed to have antigonococcal activity against strain FA1090. Primary granule proteases including proteinase 3 are required to cleave hCAP18 to LL-37 (Sorensen et al., 2001). Therefore, even if Gc phagosomes fuse with secondary granules, no LL-37 will be generated in the phagosomes that avoid primary granules. These two possibilities are not mutually exclusive, and we propose that delaying fusion of Gc phagosomes with primary granules protects Gc against early exposure to antimicrobial components housed in both primary and secondary granules, including those known to have potent antigonococcal activity.

While many pathogens can avoid the phagolysosome by modulating phagolysosomal composition, this is the first time this observation has been made for Gc. One common bacterial mechanism for phagolysosome avoidance, for instance in Legionella and Salmonella, involves the manipulation of membrane traffic via the ATPdependent secretion of bacterial proteins that target the Rab family of GTPases or Rab effectors (Urban et al., 2006). In contrast, the delay of Gc phagosome fusion with primary granules appears to be independent of active bacterial processes. This may not be surprising, as Gc does not encode secretion systems known to release proteins into 
host cells (van Ulsen et al., 2006). Instead, our results point to Gc surface composition as the mechanism directing phagosome maturation. Gc uses a variety of surface structures to manipulate host antimicrobial responses and confer resistance to antimicrobial components. Gc pili and porin have been implicated in altering PMN granule exocytosis (Bjerknes et al., 1995, Lorenzen et al., 2000, Densen et al., 1978), modifications to Gc lipooligosaccharide have been shown to increase bacterial resistance to antimicrobial peptides and proteases (Balthazar et al., 2011, Lewis et al., 2009), and the Mtr and FarAB efflux pump systems span the inner and outer membranes to export a variety of antimicrobial components out of the cell (Shafer et al., 1998, Lee et al., 1999). We speculate that as a complement to these mechanisms, Gc uses one or more surface components to direct the bacteria into a phagosomal compartment that undergoes limited, delayed fusion with primary granules. The redirection of membrane traffic could be initiated as early as during phagocytosis, since surface components on pathogens, as shown for the M and/or M-like proteins of Streptococcus pyogenes, lipophosphoglycan of Leishmania donovani, and lipoarabinomannan of Mycobacterium tuberculosis, can dictate their eventual fate inside host cells (Staali et al., 2006, Desjardins et al., 1997, Mollinedo et al., 2010, Fratti et al., 2001). Delaying of primary granule-phagosome fusion could also occur from inside the Gc phagosome, by an as-yet unappreciated mechanism. While the surface components that mediate this delay are not known, the effect is not mediated by the Gc IgA protease, which can affect the composition of Gc endosomes in non-phagocytic cells (Lin et al., 1997) (Figure 21).

Individuals infected with Gc as well as individuals with no prior history of gonorrhea produce opsonic IgG reactive against Gc surface components. However, most 
of these surface components are antigenically variable, preventing individuals from developing a protective humoral response to gonorrheal disease and contributing to the difficulty in development of a protective vaccine (Zhu et al., 2011). Development of a vaccine is a long-sought goal of the public health community and is especially pressing given the dramatic rise in multidrug-resistant Gc in the human population (Kirkcaldy et al., 2011, Ohneck et al., 2011). Importantly, our results show that IgG opsonization of Gc is capable of enhancing primary granule fusion with the Gc phagosome and reducing Gc survival inside PMNs. This observation raises the exciting possibility that if an effective antibody-based vaccine can be raised against Gc, the abundance of PMNs at the site of infection would be sufficient for host-mediated clearance of gonorrhea, whether or not the bacteria are susceptible to antibiotics.

We propose a working model to explain the remarkable capability of Gc to survive within human PMNs (Figure 22). In this model, Gc uses surface structures to influence its fate within PMNs. Selective membrane trafficking allows the majority of Gc phagosomes to delay fusion with PMN primary granules until 4-6 h post-infection. The Gc inside phagosomes that delay primary granule fusion are more likely to remain alive because they avoid degradation by primary granule proteases and membrane disruption by antimicrobial peptides. The early avoidance of primary granules is not complete, and early after internalization, a fraction of Gc are found in phagolysosomes that fuse with all classes of PMN granules. Yet some of the Gc found in these phagolysosomes remain viable, which is likely due to bacterial virulence factors that directly defend the bacteria from PMN antimicrobial components. Additionally, we speculate that the delay in primary granule fusion with the Gc phagosome allows Gc to 
adapt to the PMN intracellular environment, for instance by upregulating genes whose products confer resistance to PMN antimicrobial components. Thus Gc uses multiple, simultaneously operating mechanisms to enable its persistence and potential replication inside PMNs. Although PMNs are terminally differentiated cells, Gc has the potential to delay PMN apoptosis (Chen et al., 2011, Simons et al., 2006), which would co-opt these normally antimicrobial cells into a protected niche for Gc. We envision that the ability of Gc to persist within PMNs would facilitate long-term colonization of its obligate human host and provide increased opportunities for dissemination and transmission (Criss et al., 2012). The ability of Gc to avoid exposure to toxic PMN components reflects how effectively Gc subverts normal PMN function, which underlies the remarkable survival of Gc when confronted with the potent innate immune response of symptomatic gonorrhea. 
2.5 Figures for Chapter 2

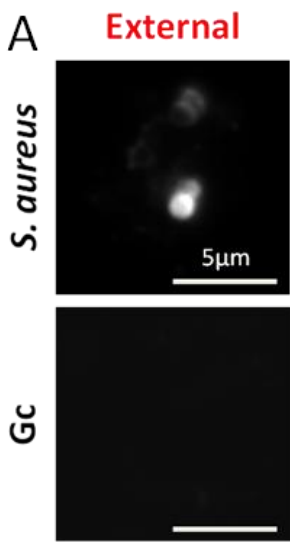

C External
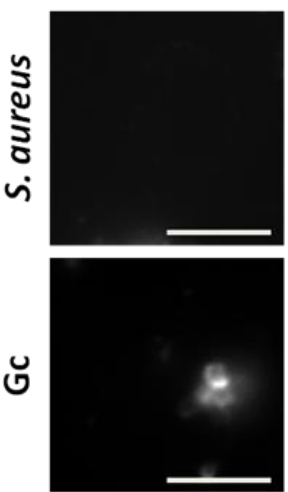

S. aureus

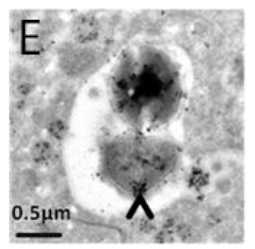

Total
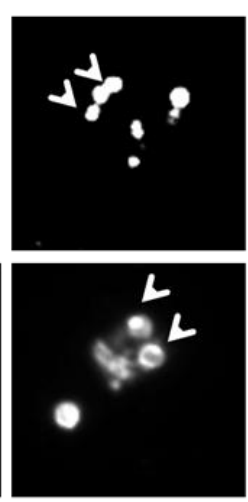

Total
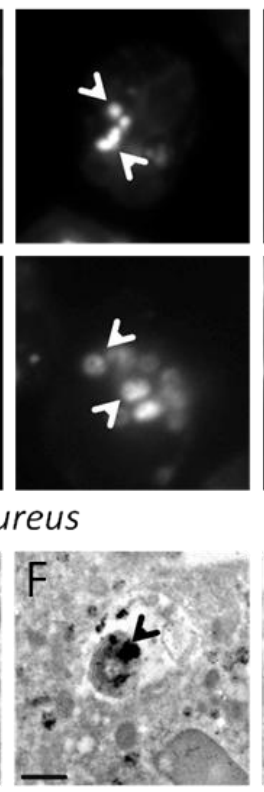
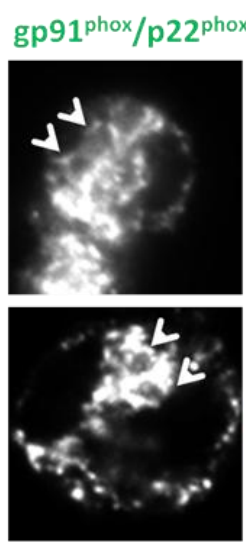

Lactoferrin
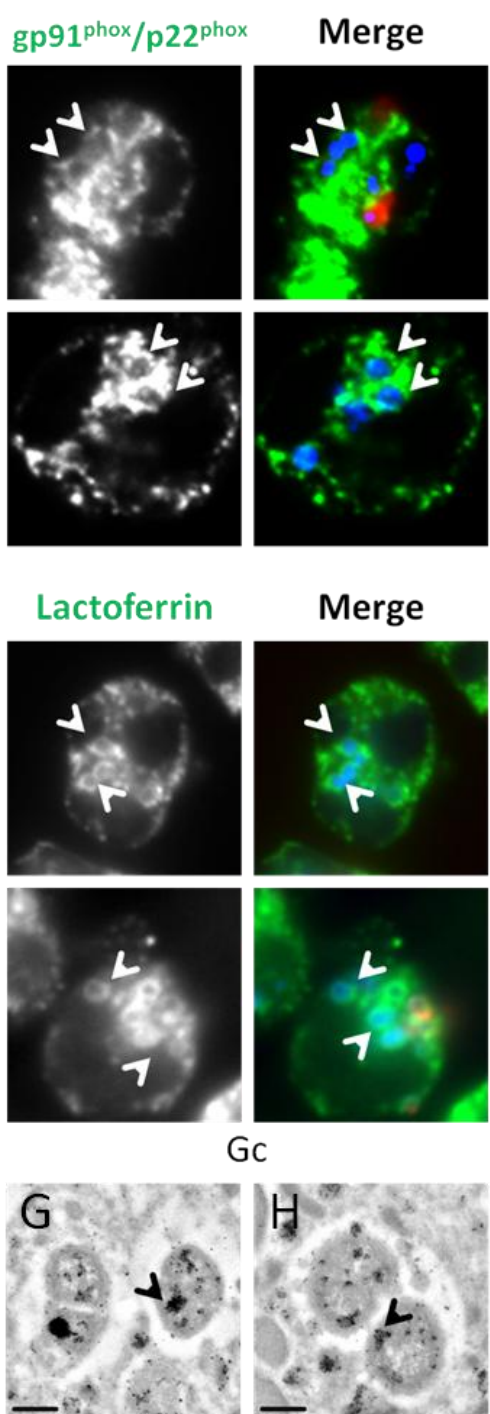

B

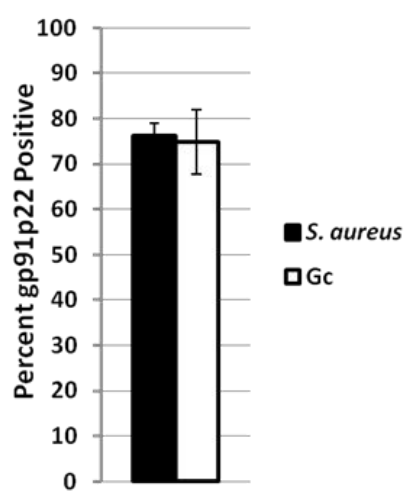

D

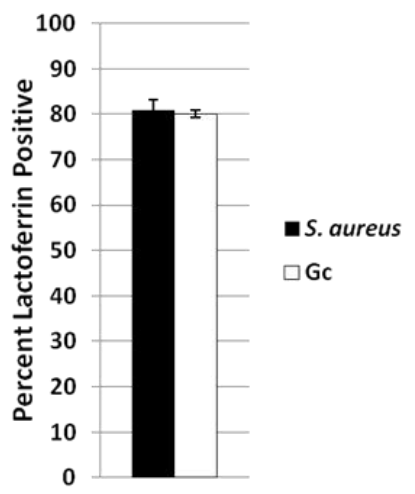

Figure 5: S. aureus and Gc phagosomes fuse with secondary and tertiary granules in primary human PMNs. 
(A-D) Adherent, IL-8 treated, primary human PMNs were infected for $1 \mathrm{~h}$ with $S$. aureus or Gc. Extracellular S. aureus and Gc appear red/blue, while intracellular S. aureus and Gc appear blue only. Both S. aureus and Gc infected PMNs were stained with antibodies against the secondary and tertiary granule proteins gp91 ${ }^{\text {phox }}$ and $\mathrm{p} 22^{\text {phox }}(\mathrm{A})$ or the secondary granule protein lactoferrin (C), which appear green. Arrowheads indicate bacterial phagosomes positive for the granule proteins. The percent of $S$. aureus and Gc phagosomes positive for gp $91^{\text {phox }}$ and $\mathrm{p} 22^{\text {phox }}$ and for lactoferrin are reported in $\mathrm{B}$ and $\mathrm{D}$, respectively. (E-H) Immuno-TEM showing phagosomes positive for lactoferrin that contain $S$. aureus $(\mathrm{E}-\mathrm{F})$ or Gc $(\mathrm{G}-\mathrm{H})$. Arrowheads indicate clusters of gold particles accumulated within bacterial phagosomes. 

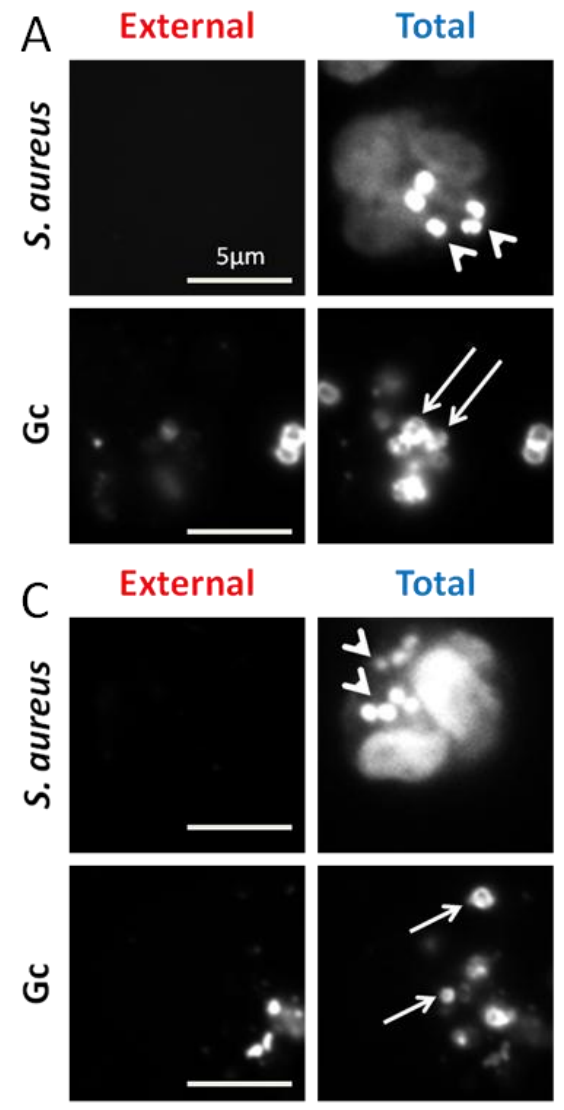

S. aureus
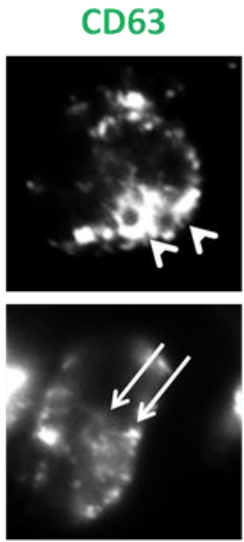

NE
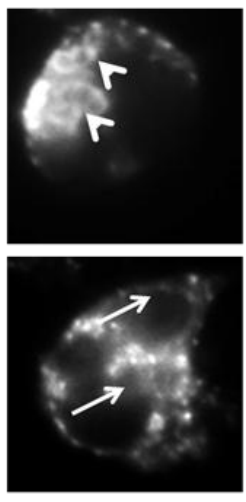

$\mathrm{Gc}$

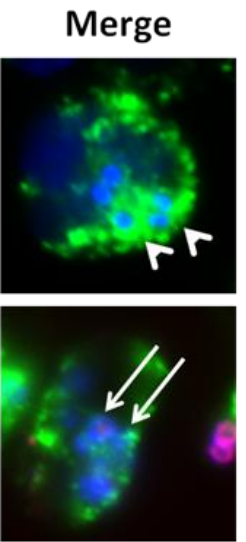

B
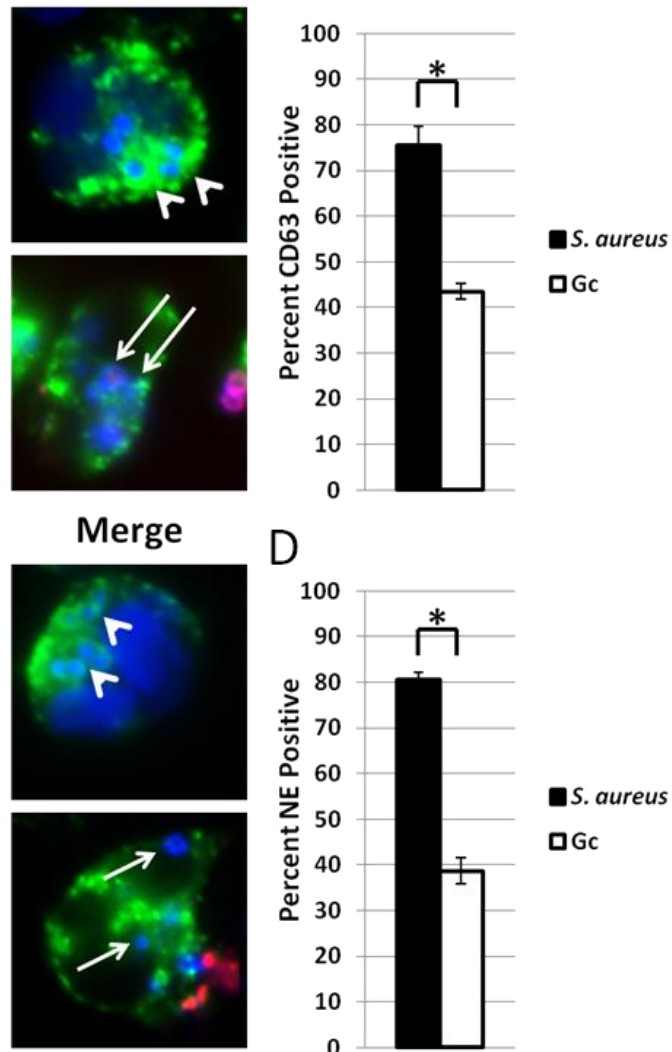

D

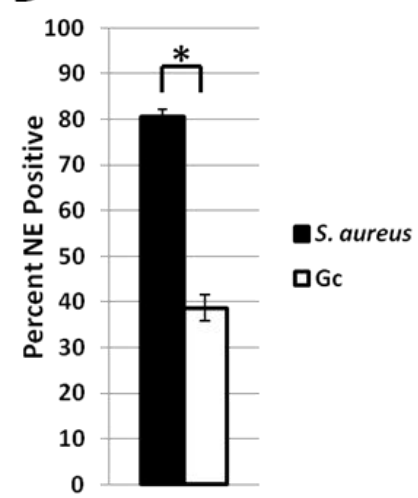

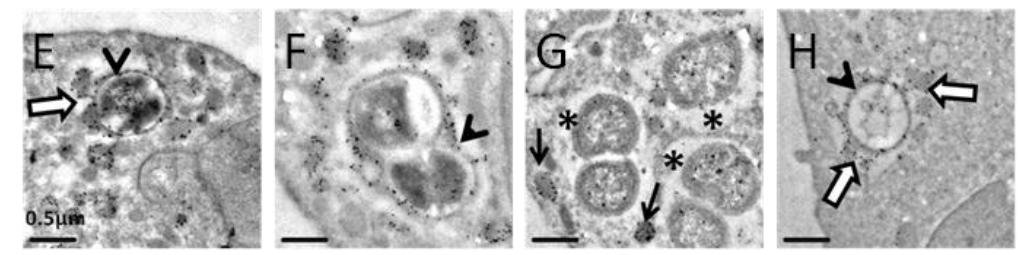

Figure 6: Gc phagosomes undergo less fusion with primary granules than S. aureus phagosomes. 
(A-D) PMNs were infected for $1 \mathrm{~h}$ with S. aureus or Gc. Extracellular S. aureus and Gc appear red/blue, while intracellular S. aureus and Gc appear blue only. PMNs were stained with antibodies against the primary granule protein CD63 (A) or neutrophil elastase (NE) (C), which appear green. Arrowheads indicate bacterial phagosomes positive for granule proteins, while arrows indicate phagosomes negative for granule proteins. The percent of $S$. aureus and Gc phagosomes positive for CD63 or neutrophil elastase are reported in $\mathrm{B}$ and $\mathrm{D}$, respectively. Asterisks indicate $P<0.05$ by Student's two-tailed $t$ test. (E-H) Immuno-TEM for myeloperoxidase (MPO) in PMNs infected with $S$. aureus (E-F) or Gc (G-H). The Gc phagosomes in H exhibit qualitiatively less MPO reactivity than the phagosome in $\mathrm{G}$ or either of the $S$. aureus phagosomes.

Arrowheads indicate a MPO-positive ring surrounding the bacteria. Open white arrows indicate MPO-positive granules associated or fusing with the phagosome. Asterisks indicate phagosomes that lack the MPO-positive ring surrounding the bacteria. Black arrows indicate granules that are not fusing with the phagosome. 


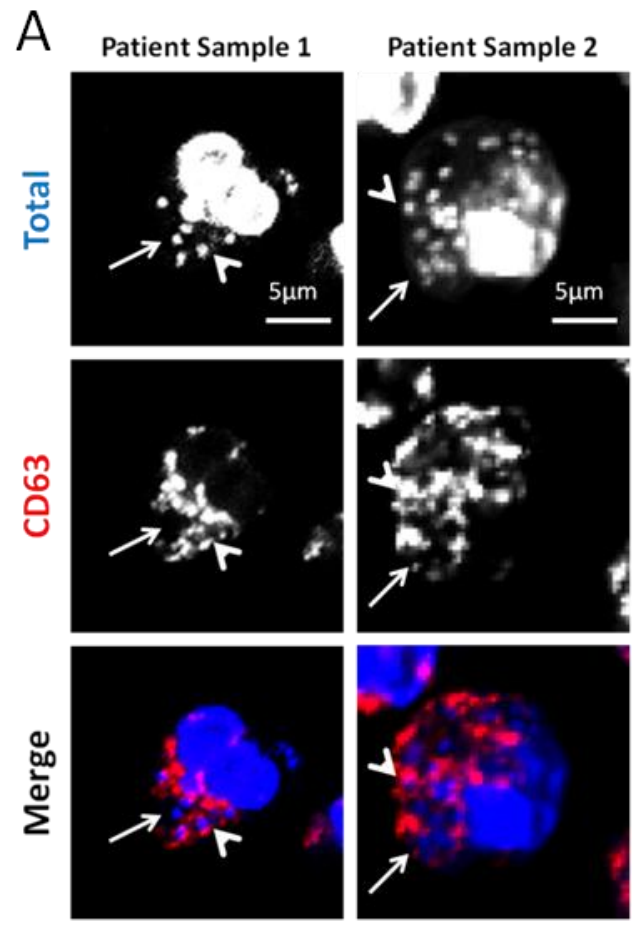

Figure 7: Gc phagosomes in PMNs from gonorrheal exudates have reduced enrichment of primary granule proteins.

Human gonorrheal exudate PMNs were stained with an anti-CD63 antibody (red) and DAPI (blue) to label total Gc. Arrowheads indicate bacteria positive for CD63 enrichment, while arrows indicate bacteria negative for CD63 enrichment. 

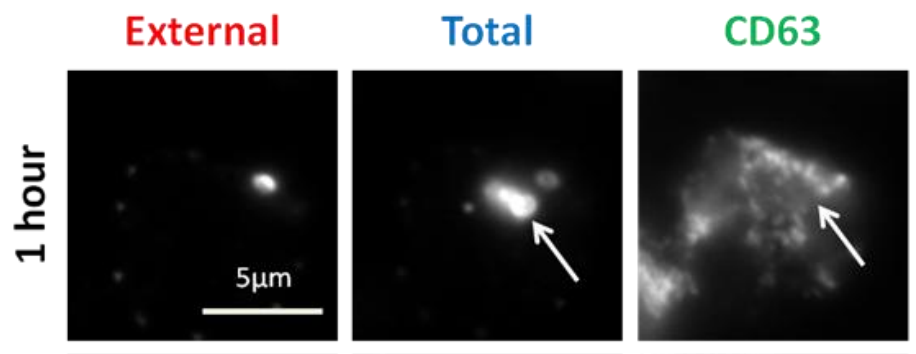

Merge
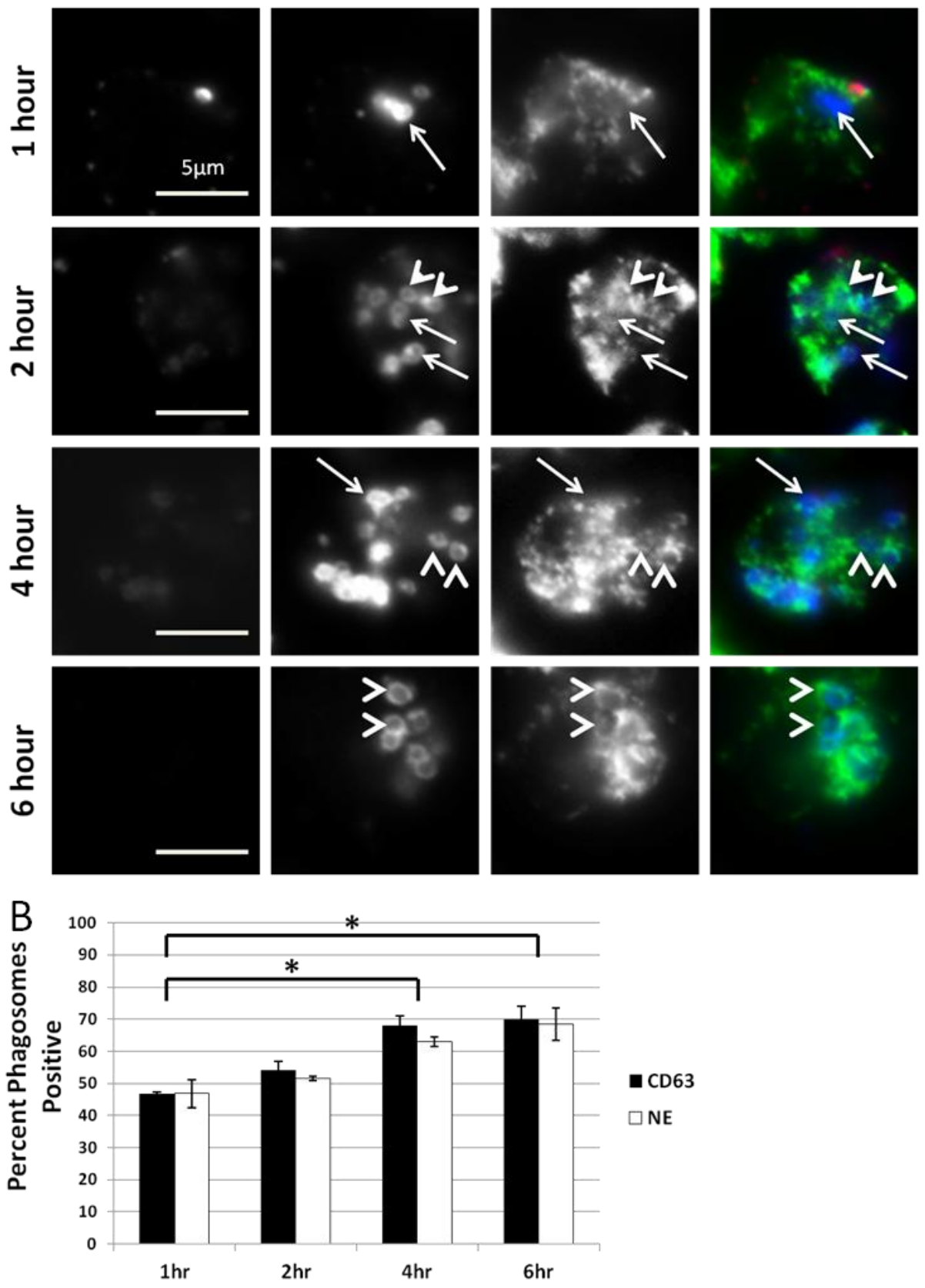

Figure 8: Gc phagosomes delay primary granule fusion. 
(A-B) PMNs were infected for 1, 2, 4, or $6 \mathrm{~h}$ with Gc. Extracellular Gc appear red/blue, while intracellular Gc appear blue only. PMNs were stained with antibodies against the primary granule protein CD63, which appears green. Arrowheads indicate bacterial phagosomes positive for granule proteins, while arrows indicate phagosomes negative for granule proteins. The average percent of Gc phagosomes positive for CD63 or neutrophil elastase from two replicate experiments are reported in B. Asterisks indicate $P<0.05$ by Student's two-tailed $t$ test. 


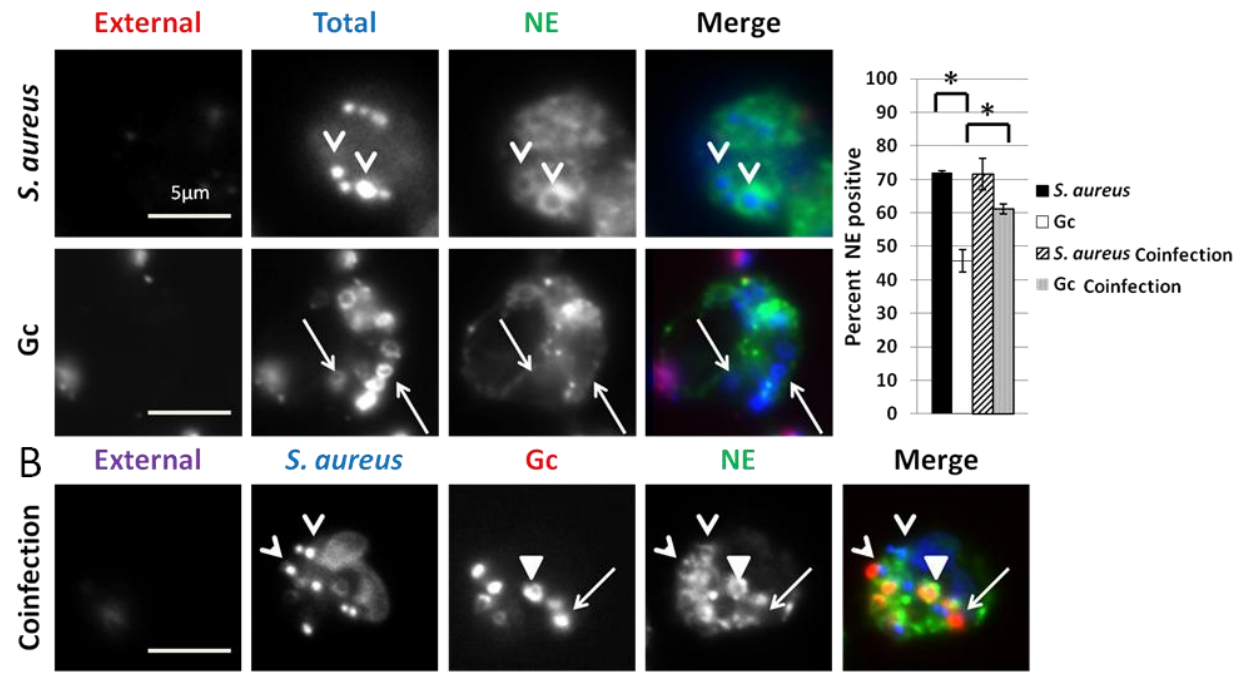

Figure 9: Gc does not globally prevent PMN primary granule fusion.

A. PMNs were infected with $S$. aureus for $30 \mathrm{~min}$ or Gc for $60 \mathrm{~min}$. Extracellular $S$. aureus and Gc appear red/blue, while intracellular S.aureus and Gc appear blue only. PMNs were stained with antibodies against the primary granule protein neutrophil elastase, which appears green. B. PMNs were coinfected with S. aureus and Gc. After 30 min of Gc infection, PMNs were infected with $S$. aureus and incubated for an additional 30 min. Extracellular S. aureus appear purple/blue and extracellular Gc appear purple/red, while intracellular S.aureus blue only and Gc appear red only. PMNs were stained with antibodies against the primary granule protein neutrophil elastase, which appears green. Arrowheads indicate $S$. aureus phagosomes positive for granule proteins, triangles indicate Gc phagosomes positive for granule proteins, and arrows indicate Gc phagosomes negative for granule proteins. The average percent of Gc and S. aureus phagosomes positive for neutrophil elastase from two replicate experiments are reported in C. Asterisks indicate $P<0.05$ by Student's two-tailed $t$ test. 


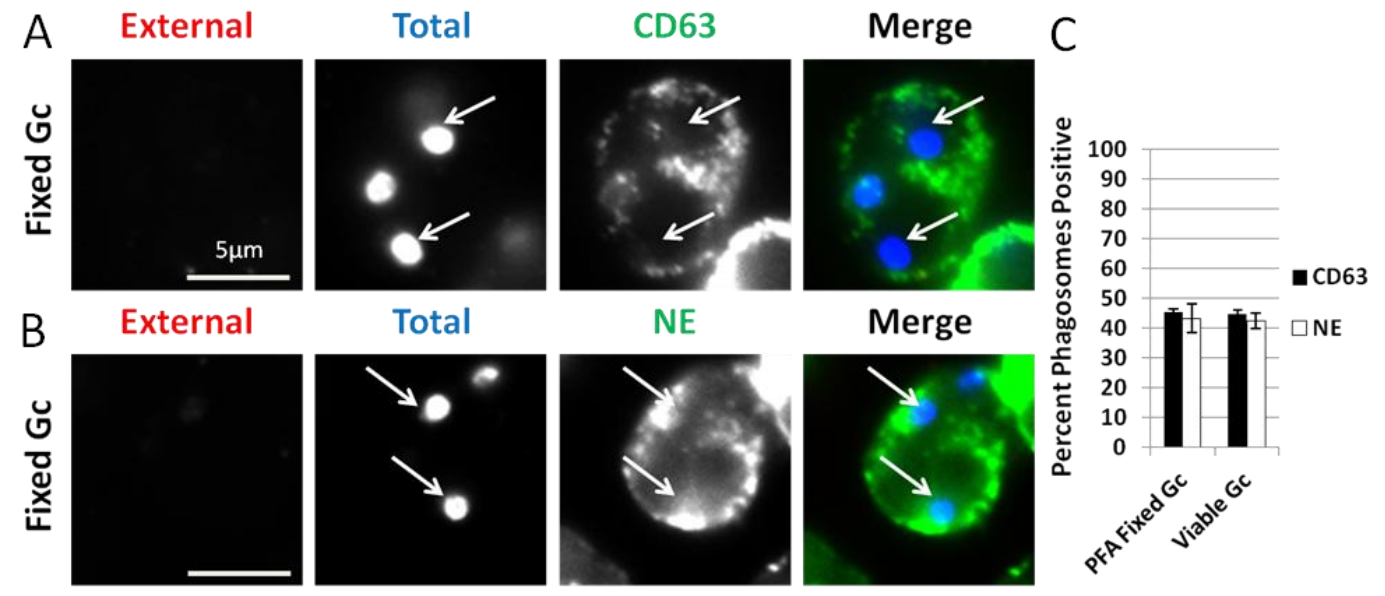

Figure 10: Active Gc processes are not required to prevent primary granule fusion with Gc phagosomes.

PMNs were exposed to paraformaldehyde (PFA)-fixed Gc for $1 \mathrm{~h}$, and intracellular and extracellular Gc were discriminated from one another along with antibodies directed against CD63 (A) or neutrophil elastase (NE) (B). Extracellular Gc appear red/blue, while intracellular Gc appear blue only, and primary granule proteins appear green. PMNs were also infected with viable Gc; these cells are not depicted but displayed the same staining pattern for neutrophil elastase and CD63 as presented in Figure 2. Arrowheads indicate bacterial phagosomes positive for granule proteins, while arrows indicate phagosomes negative for granule proteins. The percent of CD63- or NE-positive phagosomes containing viable and PFA-fixed Gc is reported in $\mathrm{C}$, respectively. 


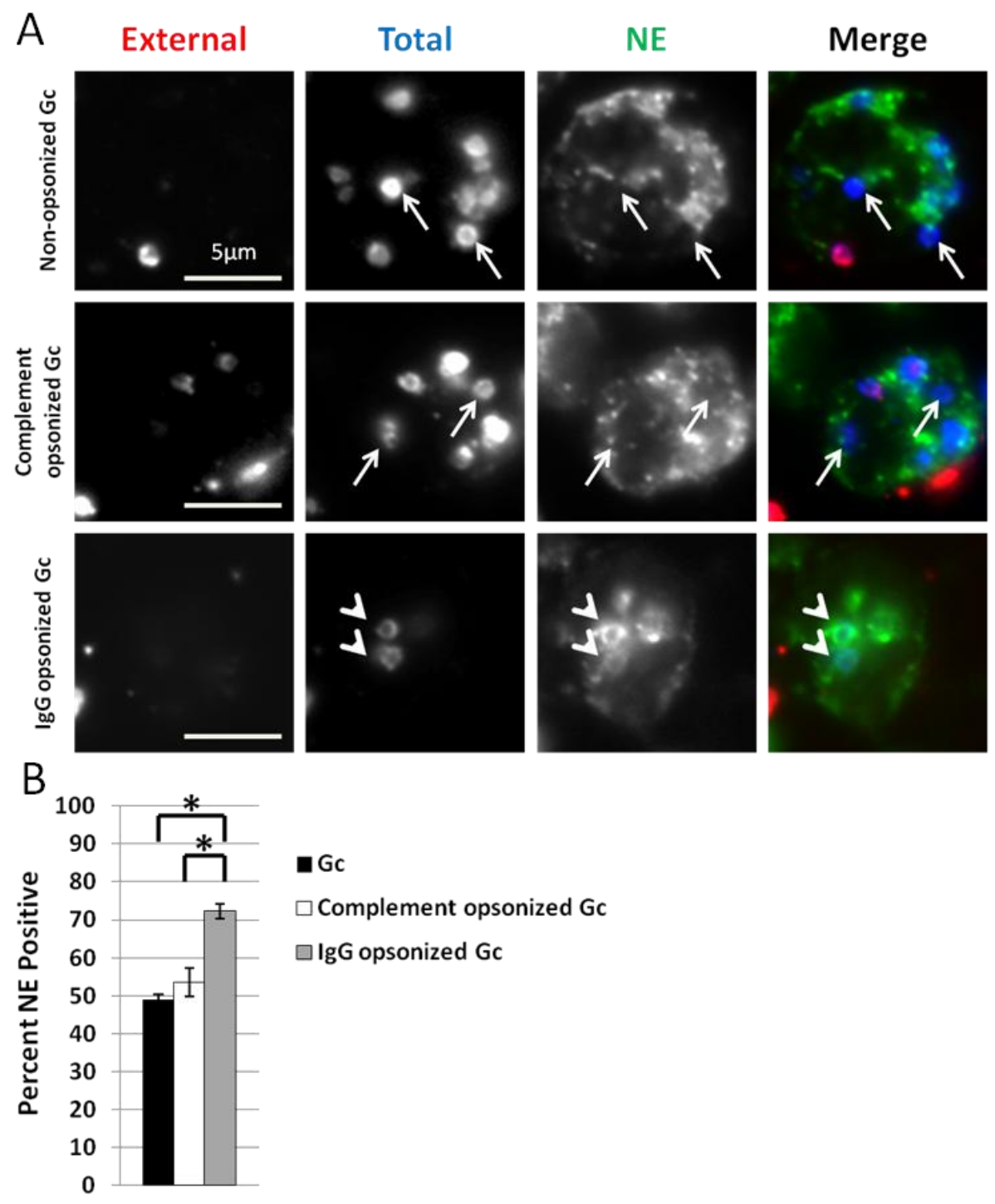

Figure 11: IgG opsonization of Gc increases primary granule fusion with Gc phagosomes.

A. PMNs were infected with non-opsonized, complement opsonized, or IgG opsonized Gc for $1 \mathrm{~h}$, and intracellular and extracellular Gc were discriminated from one another, along with an antibody directed against neutrophil elastase. Extracellular Gc appear red/blue, while intracellular Gc appear blue only, and neutrophil elastase staining appears green. Arrowheads indicate bacterial phagosomes positive for granule proteins, while arrows indicate phagosomes negative for granule proteins. The percent of neutrophil elastase-positive phagosomes containing non-opsonized, complement opsonized, or IgG opsonized Gc is reported in B. Asterisks indicate $P<0.05$ by Student's two-tailed $t$ test. 


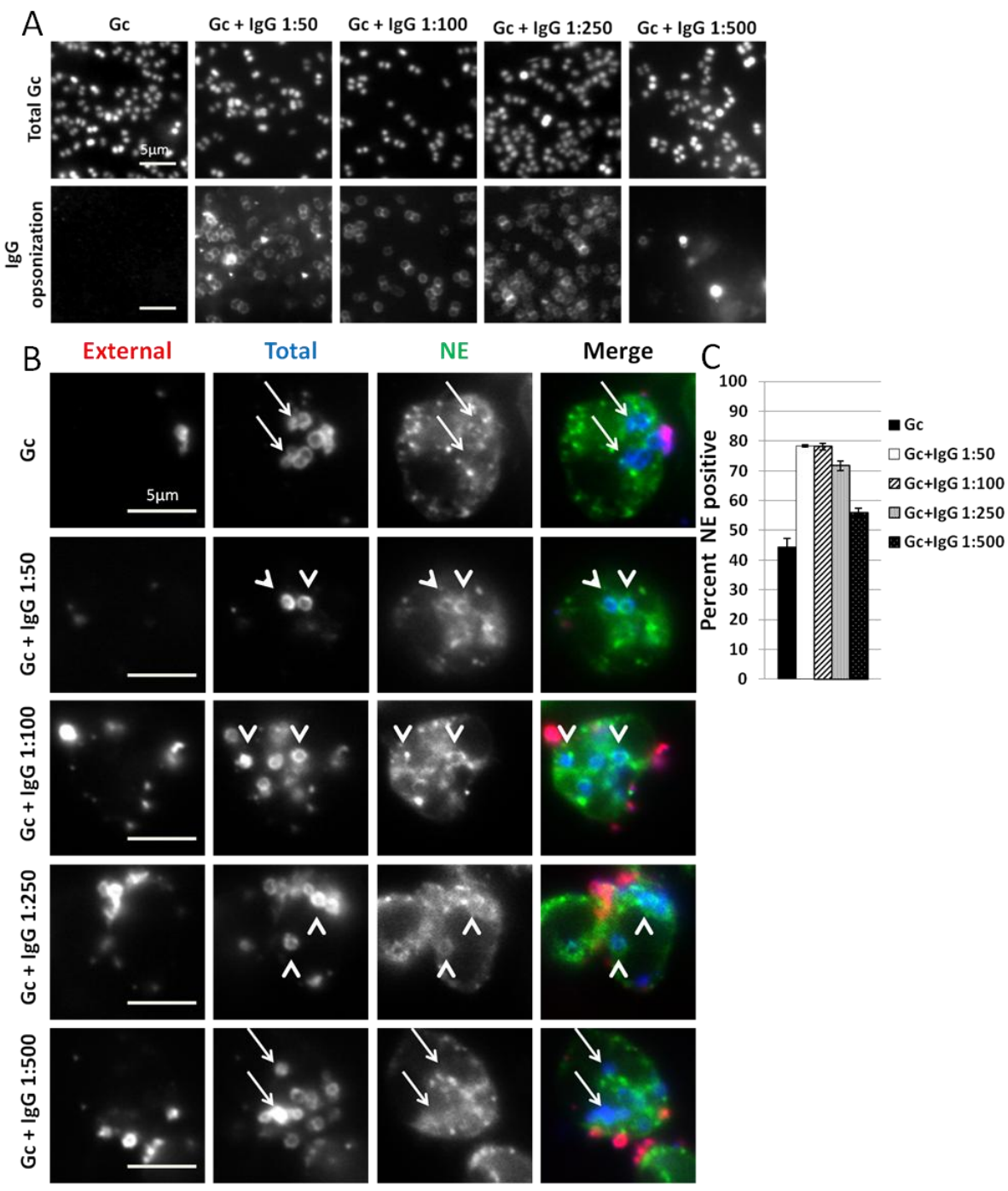

Figure 12: Titration of anti-Gc polyclonal antibody results in significantly increased trafficking of opsonized Gc to primary granule-positive phagosomes. 
A. Gc were left nonopsonized or opsonized with incremental concentrations of a polyclonal rabbit anti-Gc antibody (1:50, 1:100, 1:250, 1:500). Following opsonization Gc was labeled with DAPI to detect the total number of bacteria and incubated for $1 \mathrm{~h}$ with an Alexa Fluor-coupled goat anti-rabbit antibody to detect the amount of Gc opsonized with the polyclonal rabbit anti-Gc antibody. B. PMNs were infected with nonopsonized or IgG opsonized (1:50, 1:100, 1:250, 1:500) Gc for $1 \mathrm{~h}$, and intracellular and extracellular Gc were discriminated from one another, along with an antibody directed against neutrophil elastase. Extracellular Gc appear red/blue, while intracellular Gc appear blue only, and neutrophil elastase staining appears green. Arrowheads indicate bacterial phagosomes positive for granule proteins, while arrows indicate phagosomes negative for granule proteins. The percent of Gc phagosomes positive for neutrophil elastase is reported in $\mathrm{C}$. The percent of Gc phagosomes positive for neutrophil elastase was significantly less in PMNs infected with non-opsonized Gc compared to PMNs infected with IgG opsonized Gc (1:50, 1:100, and 1:250) as determined by Student's twotailed $t$ test $(P<0.05)$. The percent of Gc phagosomes positive for PMNs infected with IgG opsonized Gc (1:500) was significantly less than PMNs infected with IgG opsonized Gc $(1: 50,1: 100)$ as determined by Student's two tailed $t$ test $(\mathrm{P}<0.05)$ 


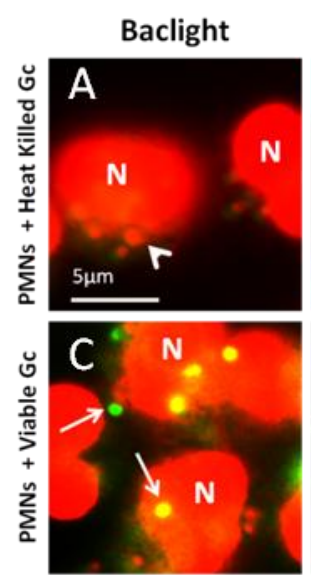

Sytox + DAPI
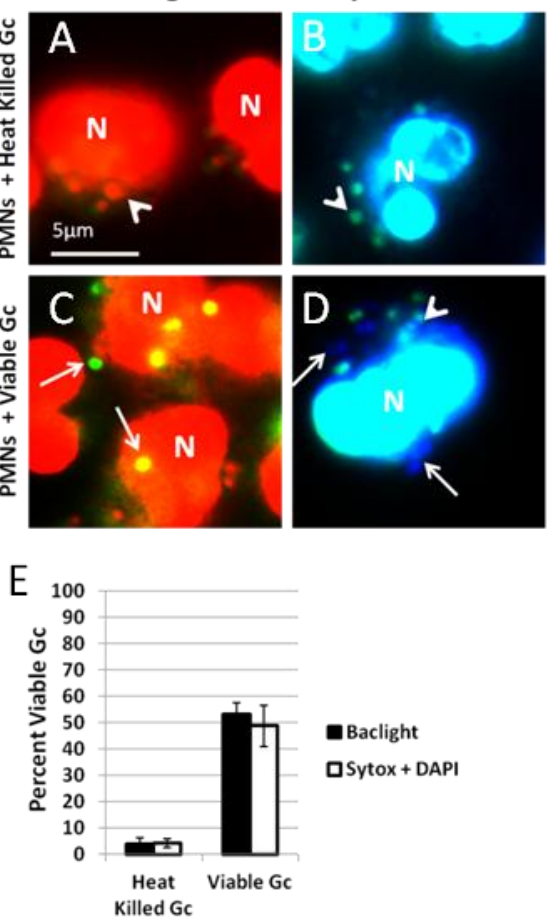

Figure 13: Sytox Green and DAPI effectively discriminate viable and nonviable Gc during PMN infection.

(A-B) PMNs were incubated with heat killed Gc for $1 \mathrm{~h}$ and processed using Baclight viability dyes (A) or Sytox Green in combination with DAPI (B). (C-E) PMNs were infected with exponentially-growing, viable Gc for $1 \mathrm{~h}$ and processed using Baclight viability dyes (C) or Sytox Green in combination with DAPI (D). In both (A) and (C) nonviable bacteria are stained with propidium iodide and appear red. (All bacteria are stained with SYTO9, but only viable bacteria appear green due to fluorescence resonance energy transfer between propidium iodide and SYTO9.) In both (B) and (D) nonviable bacteria are stained with Sytox Green and DAPI (teal) and viable bacteria are stained with DAPI only (blue). Arrowheads indicate nonviable Gc, and arrows indicate viable Gc. The percent of viable Gc discriminated using the two dye combinations is reported in E. 


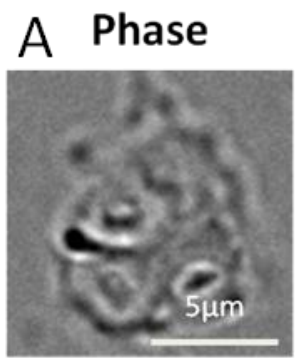

CD63 555
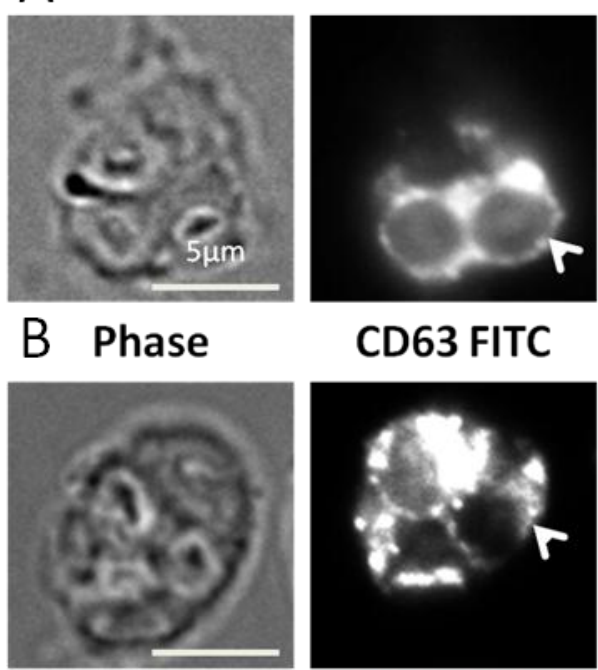

\title{
CD63 FITC
}
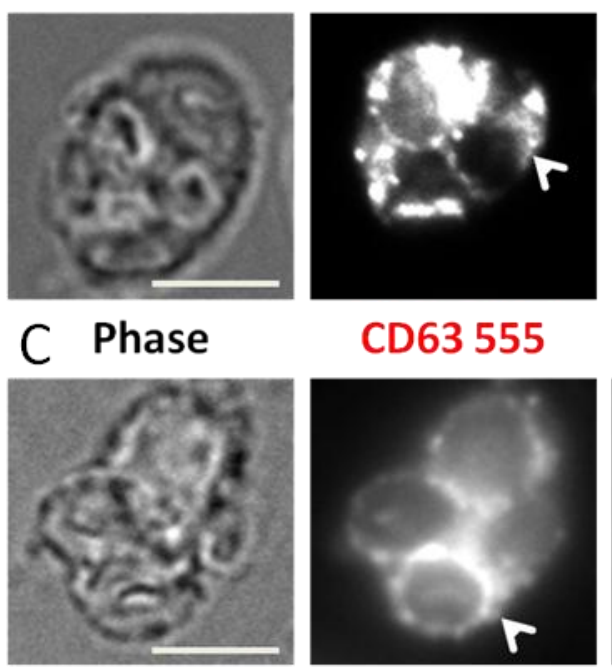

\begin{abstract}
CD63 555
\end{abstract}
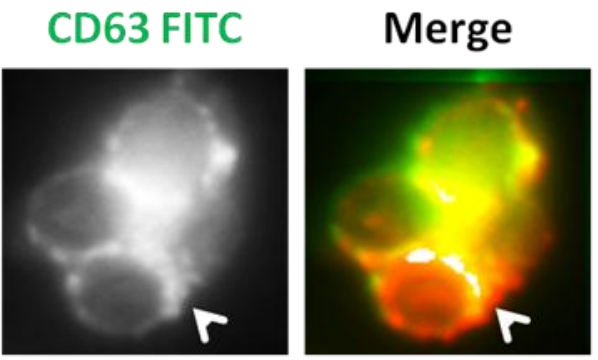

Figure 14: In the absence of fixation, an anti-CD63 antibody effectively labels primary granule and zymosan-containing phagolysosomes.

PMNs were incubated with serum-opsonized zymosan for $25 \mathrm{~min}$. Cells were left unfixed and incubated with an Alexa Fluor 555-coupled anti-CD63 antibody (A), or fixed with PFA and incubated with a FITC-coupled anti-CD63 antibody (B). C. Unfixed PMNs that had internalized opsonized zymosan were permeabilized and incubated with the Alexa 555-coupled anti-CD63 antibody. PMNs were then fixed and incubated with the FITCcoupled anti-CD63 antibody. Arrowheads indicate zymosan phagosomes positive for CD63. 


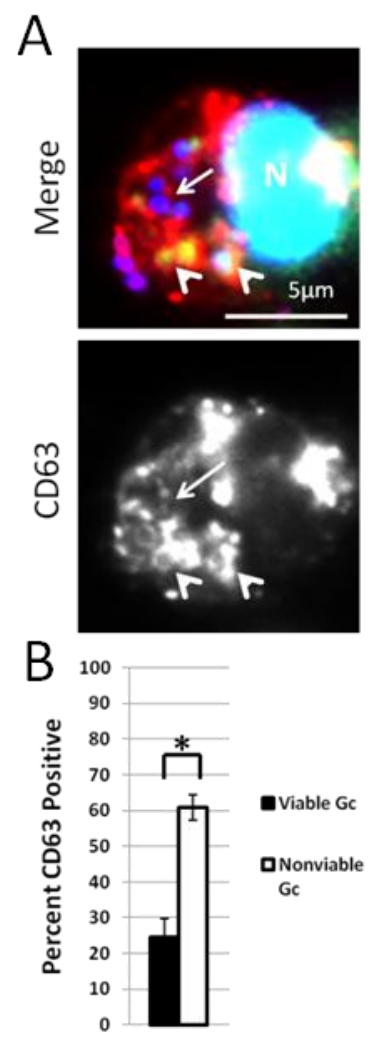

Figure 15: Increased enrichment of the primary granule protein CD63 on phagosomes containing nonviable Gc.

PMNs were infected with DAPI-labeled Gc (blue) for $1 \mathrm{~h}$. Infected PMNs were exposed to soybean lectin (purple) to detect extracellular Gc, then permeabilized and incubated with an antibody against CD63 (red) along with Sytox Green to detect nonviable Gc (green). Viable intracellular Gc appear blue, and nonviable intracellular Gc appear green/blue. A representative infected PMN is shown in A. Arrowheads indicate bacterial phagosomes positive for granule proteins, while arrows indicate phagosomes negative for granule proteins. The percent of viable and nonviable Gc residing in CD63+ phagosomes is reported in B. Asterisks indicate $P<0.05$ by Student's two-tailed $t$ test. 

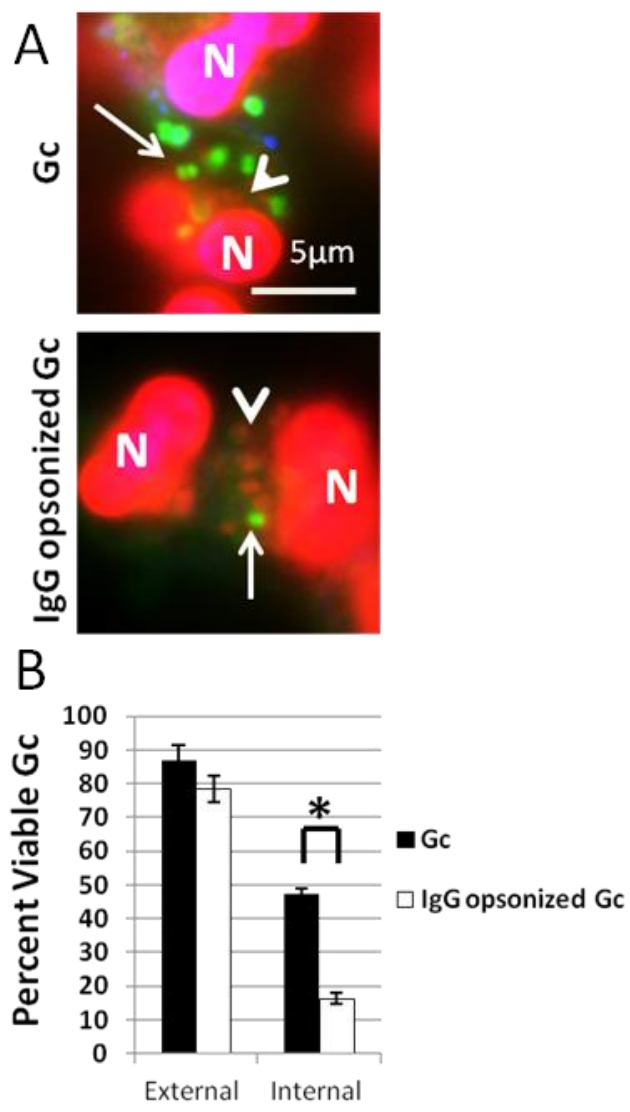

Figure 16: IgG opsonization increases primary granule fusion with Gc phagosomes resulting in decreased Gc intracellular viability.

PMNs were infected with non-opsonized or IgG opsonized Gc (A). Viable Gc (green) and nonviable Gc (red) were discriminated using Baclight viability dyes SYTO9 and propidium iodide, and extracellular Gc were labeled with soybean lectin (blue). Arrows indicate viable, intracellular Gc and arrow heads indicate nonviable, intracellular Gc. The percent of viable extracellular and intracellular bacteria is reported in B for PMNs infected with non-opsonized and IgG opsonized Gc. Asterisks indicate $P<0.05$ by Student's two-tailed $t$ test. 


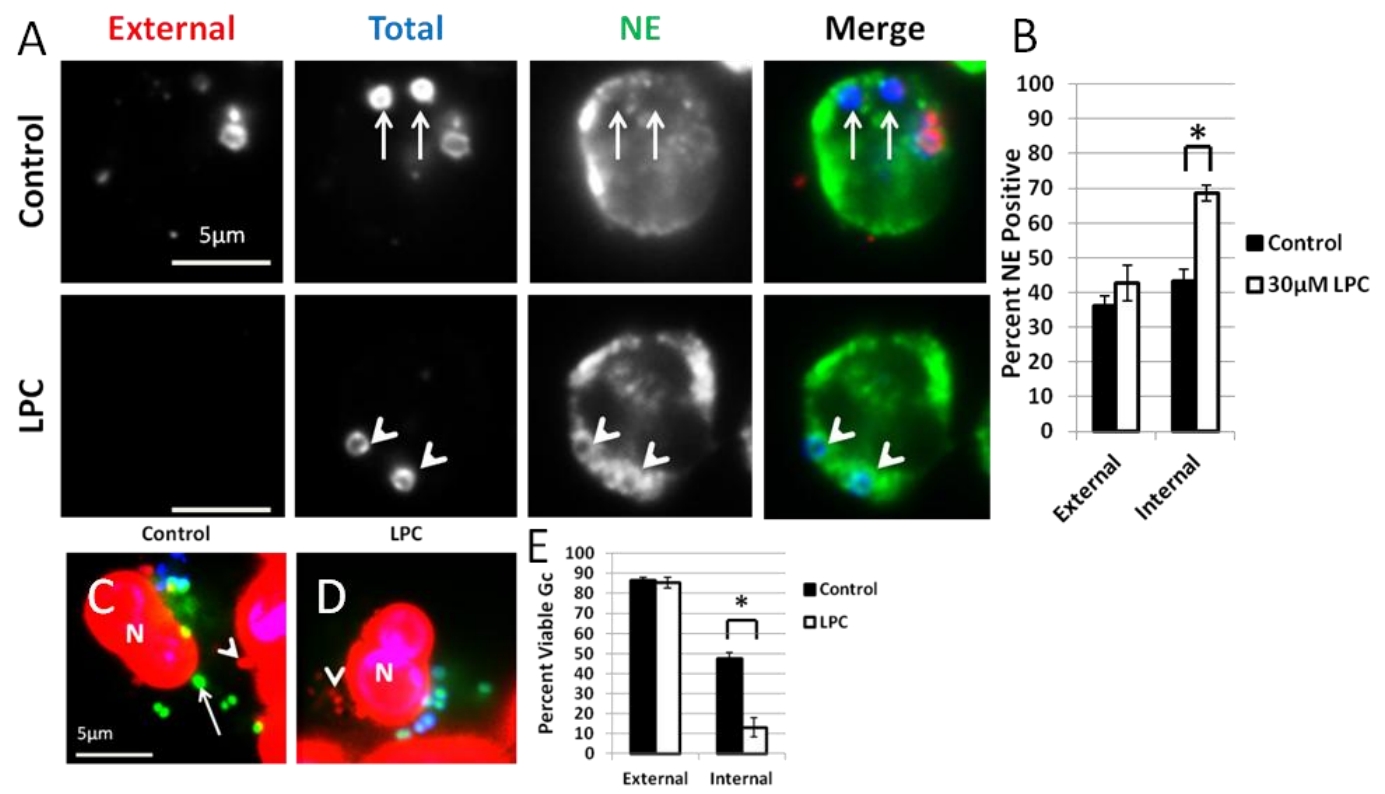

Figure 17: LPC treatment of primary human PMNs increases primary granule fusion with Gc phagosomes.

(A-B) PMNs were infected with Gc for 45 min, then given media with or without LPC. Intracellular and extracellular Gc were discriminated from one another along with an antibody raised against neutrophil elastase (NE). Extracellular Gc appear red/blue, intracellular Gc appear blue only, and neutrophil elastase staining appears green. In (A) arrowheads indicate bacterial phagosomes positive for granule proteins, while arrows indicate phagosomes negative for granule proteins. The percent of phagosomes exhibiting enrichment of neutrophil elastase with and without LPC treatment is reported in B. (C-E) PMNs were infected with Gc and left untreated (C) or treated with LPC (D). Viable Gc (green) and nonviable Gc (red) were discriminated using Baclight viability dyes SYTO9 and propidium iodide, and extracellular Gc were labeled with soybean lectin (blue). Extracellular viable Gc appear teal, intracellular nonviable Gc appear red, and intracellular viable Gc appear green. In $\mathrm{C}$ and $\mathrm{D}$ arrows indicate viable, intracellular Gc and arrow heads indicate nonviable, intracellular Gc. The percent of viable extracellular and intracellular Gc in untreated and LPC-treated PMNs is reported in E. Asterisks indicate $P<0.05$ by Student's two-tailed $t$ test. 

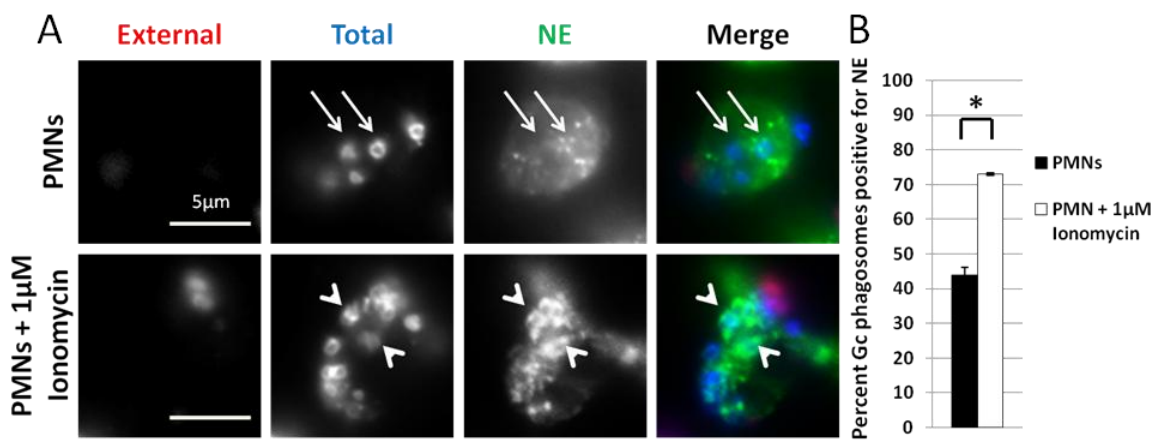

Figure 18: Ionomycin treatment of primary human PMNs increases primary granule fusion with Gc phagosomes.

(A-B) PMNs were infected for 30 min with Gc. After 30 min PMNs were treated with $1 \mu \mathrm{M}$ Ionomycin and incubated for 15 min followed by fixation and processing via immunofluorescence. Extracellular Gc appear red/blue, while intracellular Gc appear blue only. PMNs were stained with antibodies against the primary granule protein neutrophil elastase (NE), which appears green (A). Arrowheads indicate bacterial phagosomes positive for granule proteins, while arrows indicate phagosomes negative for granule proteins. The percent of Gc phagosomes positive for neutrophil elastase is reported in B. Asterisks indicate $P<0.05$ by Student's two-tailed $t$ test. 

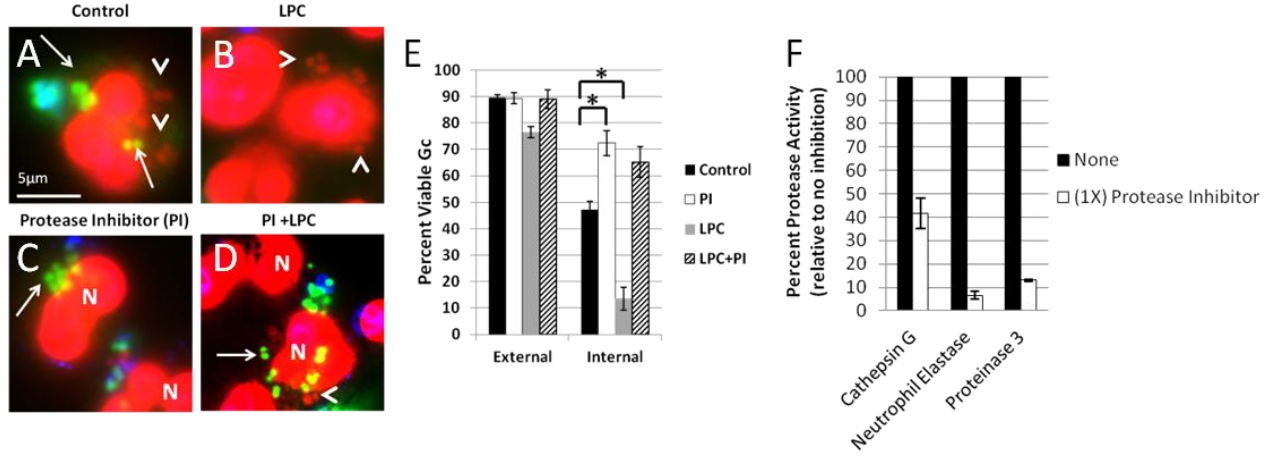

Figure 19: Ectopically increasing primary granule fusion with Gc phagosomes reduces intracellular Gc viability by a protease-dependent process.

PMNs were infected with Gc and treated with LPC (B, D) or left untreated (A, C) as described in Figure 9. Prior to infection, PMNs were treated with protease inhibitor cocktail (C, D). Viable Gc (green) and nonviable Gc (red) were discriminated using Baclight viability dyes SYTO9 and propidium iodide, and extracellular Gc were labeled with soybean lectin (blue). Extracellular viable Gc appear teal, intracellular nonviable Gc appear red, and intracellular viable Gc appear green. Arrows indicate viable, intracellular $\mathrm{Gc}$ and arrow heads indicate nonviable, intracellular Gc. The percent of viable extracellular and intracellular Gc in control PMNs, protease inhibitor treated PMNs, LPC treated PMNs, and PMNs treated with both the protease inhibitor and LPC is reported in E. Asterisks indicate $P<0.05$ by Student's two-tailed $t$ test. The percent protease activity of cathepsin G, neutrophil elastase, and proteinase 3 was compared for untreated PMNs and PMNs treated with the protease inhibitor cocktail $(\mathrm{F})$. 


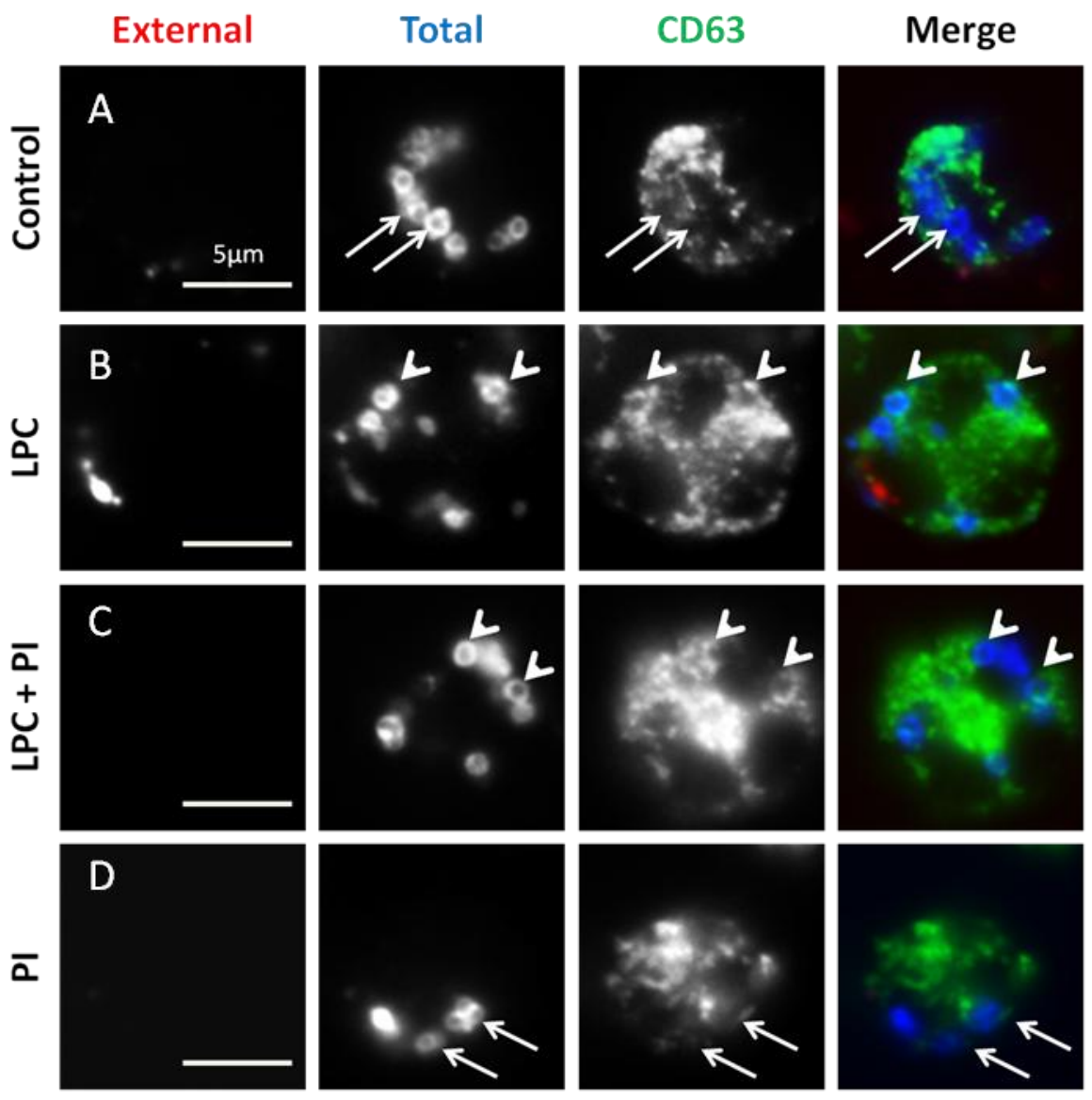

Figure 20: Protease inhibitor treatment does not affect the LPC-enhanced fusion of primary granules with PMN phagosomes.

PMNs were infected with Gc and treated with LPC (B, D) or left untreated (A, C) as in Figure 5. Prior to infection, PMNs were treated with the protease inhibitor cocktail (C, D) as in Figure 10. Immunofluorescence for CD63 and Gc was conducted as in Figure 2. Extracellular Gc appear red/blue, intracellular Gc appear blue only, and CD63 appears green. Arrowheads indicate bacterial phagosomes positive for granule proteins, while arrows indicate phagosomes negative for granule proteins. 

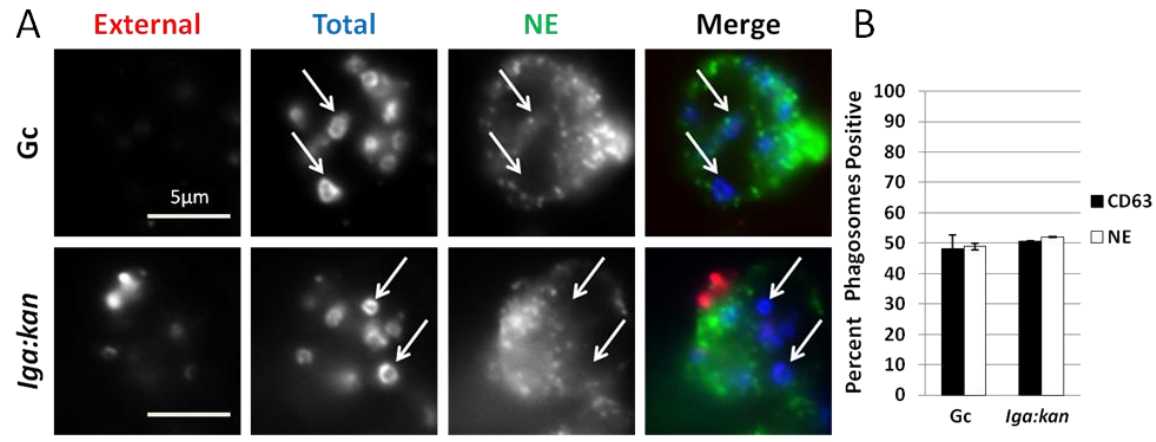

Figure 21: Gc IgA protease does not affect primary granule-phagosome fusion in PMNs.

(A-B) PMNs were infected for $1 \mathrm{~h}$ with parental or iga:kan mutant Gc and processed for immunofluorescence as in Figure 6. Extracellular Gc appear red/blue, intracellular Gc appear blue only, and neutrophil elastase (NE) appears green. Arrows indicate phagosomes negative for granule proteins. The percent of Gc and iga:kan phagosomes positive for CD63 (images not shown) or NE are reported in B. 


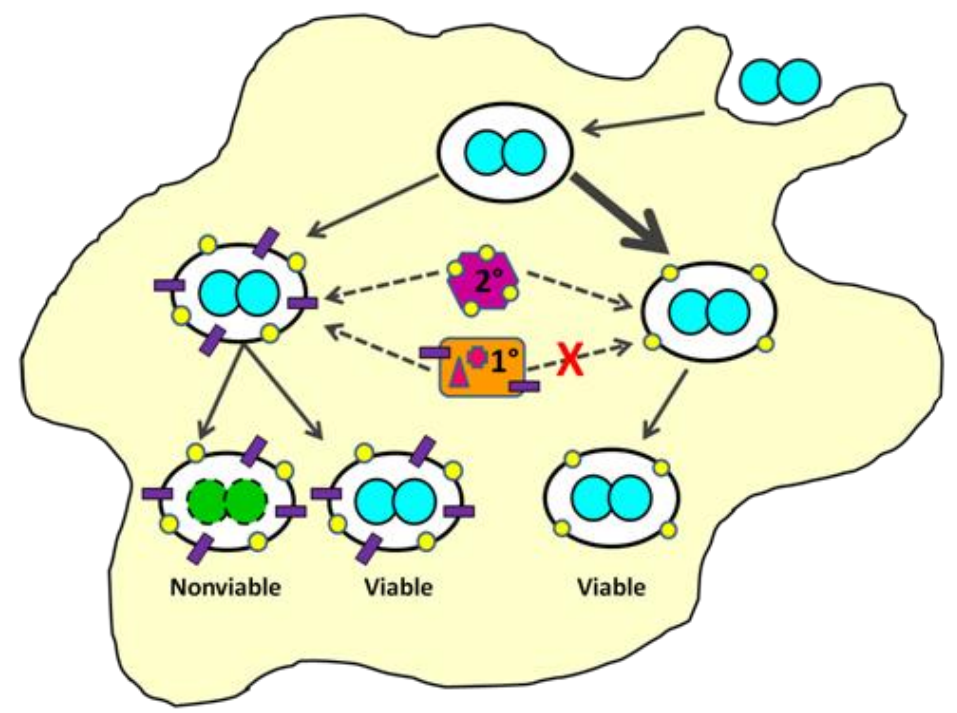

Figure 22: Model for Gc survival inside primary human PMNs. 
3. Chapter 3: Opa+ Gc have reduced survival in PMNs due to residence in a mature phagolysosomes

\subsection{Introduction}

Worldwide, there are 106 million cases of gonorrhea each year (World Health Organization, 2012). Gonorrhea remains a fixture in society and major global health problem due to the emergence of antibiotic resistant strains and lack of a protective vaccine. As a result there is increased necessity for research to understand gonorrhea pathogenesis, in order to develop new treatment options.

In response to infection with the causative agent of gonorrhea, Neisseria gonorrhoeae $(\mathrm{Gc})$, there is a large influx of neutrophils (PMNs). This results in the purulent exudate, characteristic of gonorrheal infection (Cohen et al., 1999, Evans, 1977). PMNs are professional phagocytes and a key component of the innate immune response responsible for controlling bacterial infection. PMN antimicrobial mechanisms include generation of reactive oxygen species (ROS) via NADPH oxidase, antimicrobial enzymes and peptides, and production of neutrophil extracellular traps (NETs)(Borregaard, 2010, Borregaard et al., 2007, Papayannopoulos et al., 2009). Interestingly, viable Gc can be cultured from patient exudates and primary human PMNs infected ex vivo with Gc despite the presence of these antimicrobial cells (Johnson et al., 2013b, Wiesner et al., 1980, Criss et al., 2009, Simons et al., 2005). This suggests that Gc has developed multiple mechanisms to avoid and defend against PMN mediated killing.

Opacity-associated (Opa) proteins are one prominent structure on the Gc outer membrane. Gc strains typically possess 11 opa genes, encoding for 7-9 distinct Opa proteins (Bhat et al., 1991, Dempsey et al., 1991, Connell et al., 1990). Slipped strand 
mispairing at pentameric repeats on the $5^{\prime}$ end of opa genes causes opa genes to shift in or out of frame(Stern et al., 1986, Murphy et al., 1989). As a result of this phase variation, in in vitro cultures and during infection there is a mixed population of bacteria expressing no Opa proteins, one Opa protein, or multiple Opa proteins. Opa proteins are 25-30 kDa proteins that form an eight-stranded $\beta$-barrel with four extracellular loops. The $\beta$-barrel is highly conserved in contrast to the extracellular loops, which contain variable sequence regions termed semivarible and hypervarible domains. The variability in these extracellular loops determines receptor specificity (Swanson, 1978, Vandeputte-Rutten et al., 2003, de Jonge et al., 2003, Malorny et al., 1998). Opa proteins can bind to carcinoembryonic antigen-related cell adhesion molecules (CEACAMs) and heparan sulfate proteoglycans (HSPGs) on host cells (Sadarangani et al., 2011). CEACAMs contain an Ig N-terminal domain which binds Opa proteins via protein-protein interactions (Bos et al., 1998). Of the CEACAM family of receptors, Opa proteins have been shown to bind only CEACAM1, CEACAM3, CEACAM5, and CEACAM6 (Chen et al., 1997, Chen et al., 1996, Virji et al., 1996a, Virji et al., 1996b, Gray-Owen et al., 1997a, Gray-Owen et al., 1997b, Popp et al., 1999).

PMNs express three of the CEACAM receptors known to bind Opa proteins, CEACAM1, CEACAM3, and CEACAM6 (Gray-Owen et al., 2006). Binding of Opa proteins to CEACAM receptors on PMNs is sufficient to mediate bacterial internalization in the absence of antibody or complement mediated opsonization(King et al., 1978b, Virji et al., 1986, Fischer et al., 1988). However, the signaling pathway leading to internalization varies depending on which CEACAM receptor is engaged. CEACAM1 has immunoreceptor tyrosine-based inhibition motifs and CEACAM3 has 
immunoreceptor tyrosine-based activation motifs, which upon engagement, results in either phosphatase recruitment or kinase recruitment, respectively. In contrast, CEACAM5 and CEACAM6 are glycosylphosphatidylinositol-anchored and the signaling is largely unknown (McCaw et al., 2004, Sadarangani et al., 2011). It is important to note that expression of Opa proteins is not required for PMN mediated adherence and internalization of Gc. We have demonstrated that adherent PMNs can internalize Opa- Gc (Criss et al., 2009, Ball et al., 2013). Initially, Opa+ Gc internalization is accelerated; however, after 1 hour there is no significant difference between the percent internalization of Opa+ and Opa- Gc (Criss et al., 2009, Ball et al., 2013, Smirnov et al., 2013).

In addition to mediating adherence and internalization Opa proteins influence PMN activation including generation of the oxidative burst and exocytosis (Criss et al., 2012, Johnson et al., 2011). In response to Opa engagement of CEACAM, PMNs assemble the NADPH oxidase complex in order to generate reactive oxygen species (ROS). In contrast, Opa- Gc fail to induce an oxidative burst due to incomplete assembly of the cytoplasmic subunits of the NADPH oxidase complex. Additionally, Opa- Gc can suppress PMN production of ROS induced by other stimuli due to a currently unknown mechanism (Criss et al., 2008, Smirnov et al., 2013). Increased exocytosis of primary and secondary granules is also observed in response to infection with Opa+ Gc compared to Opa- Gc (Sarantis et al., 2007). Together, these data would suggest there is a disadvantage to Opa protein expression due to the Opa protein dependent antimicrobial response generated by PMNs. In support of this, we and others have demonstrated that Opa+ Gc have decreased survival compared to Opa- Gc in human PMNs (Criss et al., 
2009, Ball et al., 2013, Rest et al., 1982, Virji et al., 1986); however, the precise mechanism leading to this survival defect is not yet defined.

There are two nonexclusive hypotheses to explain the observed survival defect of Opa+ Gc in PMNs. First, Opa proteins alter activation of PMN antimicrobial responses. Second, Opa proteins alter sensitivity to PMN antimicrobial components. Due to the ability of Opa protein expression to phase vary in Gc, we took advantage of a Gc strain with in frame deletions of all opa genes (Opaless) and Gc which constitutively expresses OpaD in the Opaless background, to address these hypotheses (Ball et al., 2013).

Here, we present data which demonstrates that Opa+ Gc have significantly reduced intracellular survival in PMNs due to signaling via CEACAM receptor engagement which triggers formation of a degradative phagolysosome. Residence in a degradative phagolysosome leads to Opa+ Gc exposure to PMN non-oxidative components which is the primary reason for reduced Opa+ Gc survival in primary human PMNs.

\subsection{Materials and Methods}

\subsubsection{Bacterial strains and growth conditions}

The Gc strains used in this study are in the FA1090 background which constitutively expresses the pilin variant 1-81-S2 due to mutation of the guanine quartet sequence upstream of pilE (Cahoon et al., 2009). The majority of experiments were conducted using Opaless and $\mathrm{OpaD}_{\mathrm{nv}}$ (Ball et al., 2013), referred to here as "Opa-" and "Opa+" Gc respectively. The strain defined as Opa- has in-frame deletions of the genes encoding the 11 opacity-associated (Opa) proteins (Ball et al., 2013). The strain defined as Opa+ was 
generated in the Opa- background and is locked for expression of OpaD due to replacement of the CTCTT repeat-rich region of FA1090 opaD with a sequence which is unable to undergo phase variation (Ball et al., 2013). Experiments involving DPI used $\triangle O p a B E G K$ Gc that were predominantly Opa- or Opa+ (Ball et al., 2013), as confirmed using immunoblotting (data not shown). Piliated Gc was routinely grown on Gonococcal Medium Base (Difco) plus Kellogg's supplements (Kellogg et al., 1963) for $20 \mathrm{~h}$ at $37^{\circ} \mathrm{C}$ in $5 \% \mathrm{CO}_{2}$. Gc was grown to exponential phase via successive rounds of bacterial growth in rich liquid medium (GCBL) and used to infect PMNs as previously described(Criss et al., 2008). Gc was labeled with $5 \mu \mathrm{g} \mathrm{ml}^{-1}$ carboxyfluorescein diacetate succinimidyl ester (CFSE) for $20 \mathrm{~min}$ at $37^{\circ} \mathrm{C}$. In order to coat the Gc surface with the $\mathrm{N}$ domain of CEACAM $1,2 \times 10^{8} \mathrm{Gc}$ were rotated at $37^{\circ} \mathrm{C}$ for $1 \mathrm{~h}$ in $1 \mathrm{ml}$ rich liquid medium containing $6 \mu \mathrm{g} / \mathrm{ml}$ CEACAM $1 \mathrm{~N}$ domain.

\subsubsection{N-domain CEACAM1 Purification}

E. coli strain MC1067 transformed with pGEX-2V plasmid containing the $\mathrm{N}$-terminal 107 codons of human CEACAM1 (N-CEACAM1) was a kind gift of Alena Fedarovich (Medical University of South Carolina). A tobacco etch virus protease (TEV) cleavage domain was introduced between GST and N-CEACAM1. GST-TEV-N-CEACAM1 was purified from IPTG-induced E. coli by passage of the bacterial lysate over a glutathione column. The GST tag was then cleaved using TEV, and N-CEACAM1 was purified from GST and TEV using a Sephadex S-200 gel-filtration column. Further details of NCEACAM1 expression and purification are forthcoming (J. Martin, L. Ball, T. Solomon, A. Criss, and L. Columbus, manuscript in preparation). 


\subsubsection{PMN isolation}

Venous blood was collected from healthy human subjects after receiving written informed consent. All human subject research was conducted in accordance with a protocol approved by the University of Virginia Institutional Review Board for Health Sciences Research (IRB-HSR). PMNs were isolated as previously described (Stohl et al., 2005 ) and resuspended at $1 \times 10^{7}$ cells per $\mathrm{ml}$ in Dulbecco's PBS (without calcium and magnesium; Thermo Scientific) containing $0.1 \%$ dextrose. PMNs were kept on ice until use within $2 \mathrm{~h}$ of preparation. PMN morphology was assessed by phase-contrast microscopy and indicated the PMN preparations contained approximately $95 \%$ PMNs.

\subsubsection{Gc survival in adherent, IL-8 treated PMNs}

Acid washed glass coverslips were coated for $1 \mathrm{~h}$ at $37^{\circ} \mathrm{C}$ with $50 \%$ pooled human serum (Sigma) in PBS and washed in PBS prior to PMN addition. PMNs were diluted into RPMI (Mediatech) containing 10\% fetal bovine serum (Thermo Scientific) and 10nM IL8 (R\&D Systems). $10^{6}$ PMNs were added to each serum coated coverslip and incubated for $1 \mathrm{~h}$ at $37^{\circ} \mathrm{C}, 5 \% \mathrm{CO}_{2}$ to promote $\mathrm{PMN}$ attachment as previously described (Johnson et al., 2013b). PMNs were infected with viable exponential-phase Gc at a multiplicity of infection of 1-5 bacterial colony forming units (CFU) per PMN as described in (Criss et al., 2009). Detection of viable and nonviable Gc associated with PMNs using Life Technologies Baclight Viability Dyes (Life Technologies) was conducted as previously described (Johnson et al., 2013a). Protocols describing the detailed use of fluorescent dyes to detect the viability of bacteria inside and outside of host cells are available in Appendix A (Johnson et al., 2013a). Images were acquired within 30 min of mounting coverslips. For each experiment, a minimum of 100 bacteria were analyzed for viability. 
Gc survival after exposure to adherent PMNs was conducted as previously described (Stohl et al., 2005).

\subsubsection{Opa- and Opa+ Coinfection}

_PMNs were infected with unlabeled Opa- Gc and CFSE labeled Opa+ Gc at a

multiplicity of infection of 1-2 CFU per PMN as described above. Cells were incubated 1h at $37^{\circ} \mathrm{C}$. Coinfected PMNs were fixed with $4 \%$ PFA in PBS for 15 min.

\subsubsection{Immunofluorescence}

PMNs infected for indicated times with Opa- or Opa+ Gc were fixed with 4\% PFA in PBS for 15 min. External and internal Gc were distinguished using a polyclonal rabbit anti-Gc antibody with and without permeabilization, as previously described (Criss et al., 2009). For experiments evaluating recruitment of PMN secondary granules, Gc was labeled prior to infection with CFSE to stain the total Gc population as previously described (Johnson et al., 2013b) and external Gc were detected using the polyclonal rabbit anti-Gc antibody as described above. Cells were then permeabilized using a 1:1 ratio of acetone and methanol. Post permeabilization, PMN secondary granules were recognized using a polyclonal rabbit anti-lactoferrin antibody (MP Biomedicals) and PMN primary granules were recognized using monoclonal antibodies against neutrophil elastase (AHN-10) (Millipore). For experiments evaluating recruitment of Hck, Gc was labeled prior to infection with CFSE to stain the total Gc population as previously described above. Intracellular Gc were identified by staining for PMN secondary granules using monoclonal antibodies against p22 $2^{\text {phox }}$ (44.1) and gp91 ${ }^{\text {phox }}$ (54.1) (both Santa Cruz Biotechnology) as previously described (Johnson et al., 2013b). Staining for Hck was conducted using a polyclonal rabbit anti-hck antibody (Santa Cruz Biotechnology). Cells 
were incubated with Alexa Fluor-coupled goat anti-rabbit or goat anti-mouse antibodies (Life Technologies) following primary antibody incubation. Coverslips were mounted using Flouromount $\mathrm{G}$ (Southern Biotech) with $2.5 \mathrm{mg} \mathrm{ml}^{-1}$ propyl gallate (Acros Organics). For each experiment, 50-200 intracellular bacteria were analyzed for enrichment of PMN granule proteins. Bacterial phagosomes were classified as positive for PMN granule proteins if antibody staining surrounded $\geq 50 \%$ of the bacterial circumference.

\subsubsection{PMN oxidative burst}

Generation of reactive oxygen species from PMNs exposed to Opa+ Gc was measured by luminol-dependent chemiluminescence as previously described (Criss et al., 2008)using a Victor 3 multilabel plate reader (Perkin-Elmer). Luminescence was measured in 1s intervals every $3 \mathrm{~min}$ for $60 \mathrm{~min}$.

\subsubsection{PMN Treatments}

PMNs were infected with Gc for 60, 90, or 120min and inhibitors were present for the entire infection unless otherwise noted.

3.2.8.1 Cytochalasin D: PMNs were treated with $10 \mu \mathrm{g} / \mathrm{ml}$ cytochalasin D (CytoD; Sigma Aldrich) for 10 min prior to addition of Gc and for the duration of the infection.

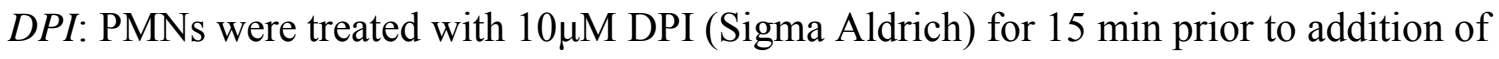
Gc and for the duration of the infection.

3.2.8.2 Protease Inhibitors: PMNs were incubated with 1X Protease Inhibitor Cocktail Set V (Calbiochem) for 15 min prior to infection with Gc as previously described (Johnson et al., 2013b). PMNs were infected with Gc for 60 or 90 min. 
3.2.8.3 Signaling Inhibitors: PMNs were incubated at $37^{\circ} \mathrm{C}$ for $30 \mathrm{~min}$ prior to infection with $30 \mu \mathrm{M}$ PP1 or $10 \mu \mathrm{M}$ LY294002. PMNs were incubated at $37^{\circ} \mathrm{C}$ for $20 \mathrm{~min}$ prior to infection with $50 \mu \mathrm{M}$ piceatannol or $68.8 \mu \mathrm{M}$ EHT1864. PMNs were incubated at $37^{\circ} \mathrm{C}$ for 30 min prior to infection with $30 \mu \mathrm{M}$ PP1 or $10 \mu \mathrm{M}$ LY294002. PMNs were incubated at $37^{\circ} \mathrm{C}$ for 10 min prior to infection with $100 \mathrm{nM}$ wortmannin. PMNs were infected with Gc for 30 or $60 \mathrm{~min}$.

3.2.8.4 Src-Family Activator: PMNs were incubated at $37^{\circ} \mathrm{C}$ for $15 \mathrm{~min}$ prior to infection with $0.5 \mathrm{mg} / \mathrm{ml}$ Src-Family Activator (Santa Cruz Biotechnology). PMNs were infected with Gc for 60 min.

\subsubsection{Antimicrobial Protein Susceptibility}

3.2.9.1 LL-37: Gc was incubated at $37^{\circ} \mathrm{C}$ for $45 \mathrm{~min}$ with LL-37 (gift from William Shafer) $(0-3 \mu \mathrm{g} / \mathrm{ml})$ in $0.5 \mathrm{X}$ GCBL and bacterial survival was calculated as previously described (Ball et al., 2013).

3.2.9.2 Cathepsin G: Cathepsin G (MP Biomedicals) was dialysed for 12 hours at $4{ }^{\circ} \mathrm{C}$ in distilled water prior to infection using SpectraPor dialysis membrane MWCO 3,500 (Spectrum Labs) to remove acetate. Gc was incubated at $37^{\circ} \mathrm{C}$ for $60 \mathrm{~min}$ in the presence of dialysed Cathepsin $\mathrm{G}(0-3 \mu \mathrm{g} / \mathrm{ml})$ in $0.1 \mathrm{X}$ GCBL. Bacterial survival was calculated as previously described (Ball et al., 2013).

\subsubsection{Image acquisition}

Images were acquired on a Nikon Eclipse E800 with Hamamatsu Orca-ER digital camera using Openlab software and processed in Adobe Photoshop CS5. Images showing

staining for hck were acquired on an LSM-700 Confocal microscope using Zen software 
and processed in Adobe Photoshop CS5. For consistency, images depicting total bacteria, including bacteria labeled with CFSE, were false colored blue.

\subsubsection{Statistics}

Experimental values are expressed as a mean \pm standard error of the mean for three replicate experiments (unless otherwise noted), performed with different donors' PMNs for each assay. A Student's two-tailed t-test was used to determine significance for each assay. A $p$ value of less than 0.05 was considered statistically significant.

\subsection{Results}

\subsubsection{Opa protein expression reduces Gc internal survival in PMNs due to engagement of CEACAM receptors}

In our previous work, we demonstrated Opa+ Gc are more sensitive to killing by

PMNs. To begin to understand the mechanism of Opa+ Gc sensitivity to PMNs, we measured the viability of intracellular and extracellular Gc after infection of adherent, IL8 primed human PMNs, using Baclight viability dyes.

Opa+ Gc had a significant reduction in internal survival in PMNs compared to Opa- Gc. Opa+ Gc also exhibited a reduction in external survival compared to Opa- Gc; however, the intracellular survival defect was more substantial (Figure 23A-C). In support of this observation, treatment of PMNs with Cytochalasin D to inhibit phagocytosis prior to infection with Opa- or Opa+ Gc significantly rescued Opa+ Gc survival to levels observed for Opa- Gc (Figure 23D). Previous studies from our lab indicate Opa+ and Opa- Gc are internalized by PMNs over this time course to the same extent (Ball et al., 2013, Smirnov et al., 2013). Therefore, the percent of Gc internalization does not account for observed differences in survival. From these data we 
conclude that the observed survival defect of Opa+ Gc in PMNs is primarily intracellular. Our subsequent experiments focused on the mechanism responsible for this intracellular survival defect.

Opa proteins are known to bind CEACAMs on the surface of host cells. Specifically, gonococcal OpaD has been shown to bind to CEACAM1 (Fulcher, 2004) and results from our lab indicate binding to CEACAM3 (manuscript in preparation). Therefore, to determine if the observed Opa+ Gc survival defect in PMNs is dependent on CEACAM engagement, we coated Opa+ Gc with the N-domain of CEACAM1 prior to infection of PMNs. Coating Gc with the $\mathrm{N}$ domain of CEACAM1 significantly reduced the PMN oxidative burst in a dose dependent manner, demonstrating the ability of soluble N-CEACAM1 to block Opa-CEACAM interactions (Figure 25). We also observed a reduction in Opa+ Gc internalization due to blocking Opa-CEACAM interactions (Figure 24). However, $45 \%$ of Opa+ Gc were still internalized in the absence of Opa-CEACAM interactions permitting us to assess differences in intracellular viability. When coated with N-CEACAM1, the intracellular viability of Opa+ Gc was significantly increased compared with uncoated Opa+ Gc alone (36\% vs 10\%, respectively) (Figure 24A-C). From these data, we conclude that the observed internal survival defect of Opa+ Gc in PMNs is dependent on Opa engagement of CEACAM(s) and blocking this interaction increases the intracellular survival Opa+ Gc.

\subsubsection{Increased maturation of phagosomes containing Opa+Gc}

We previously showed that phagosome maturation affects Gc viability in PMNs (Johnson et al., 2013b). In order to assess if Opa protein expression influences phagosome maturation in PMNs, adherent, IL-8 primed PMNs were infected with Opa+ 
or Opa- Gc for $1 \mathrm{hr}$ and phagosome maturation was observed via immunofluorescence.

The Gc phagosome was classified as enriched for a certain granule class, if the staining for an antibody directed against a protein in that PMN granule class surrounded $\geq 50 \%$ of the bacteria circumference. Solid rings of lactoferrin staining were observed for both intracellular Opa+ and Opa- Gc phagosomes, indicating both were enriched for secondary granules (Figure 26A and C). Interestingly, rings of NE staining were observed surrounding Opa+ Gc, while in contrast, Opa- Gc phagosomes showed a significant reduction in enrichment of this primary granule protein (Figure 26B and D). From these observations, we conclude that Opa protein expression directs Gc to a mature phagolysosome which has fused with both primary and secondary granules.

\subsubsection{Opa+ Gc residence in degradative phagolysosomes exposes Gc to PMN non- oxidative components which mediate killing}

Mature PMN phagolysosomes contain both a full array of non-oxidative antimicrobial proteins and peptides and also the components of the NADPH oxidase enzyme to generate ROS. We sought to determine the contribution of these oxidative and non-oxidative components to the reduced survival of Opa+ Gc inside PMNs. Expression of Opa proteins induces a strong oxidative burst in contrast to Opa- Gc which fails to induce a detectable oxidative burst in PMNs (Ball et al., 2013, Smirnov et al., 2013). In order to directly test the role of ROS in killing Opa+ Gc by PMNs, PMNs treated with diphenyleneiodonium (DPI) to inhibit production of ROS or were left untreated prior to infection with Opa+ Gc. Treatment with DPI did not rescue Opa+ Gc survival in PMNs (Figure 27). We conclude that even in the presence of a potent PMN oxidative burst, ROS is not required for PMN mediated killing of Gc. These data are in agreement with 
previous reports indicating that PMN killing of Gc is primarily non-oxidative (Criss et al., 2009, Seib et al., 2005, Rest et al., 1982, Frangipane et al., 1992, Wu et al., 2009).

Both PMN primary and secondary granules contain non-oxidative components including LL-37, antimicrobial peptides, and proteases such as cathepsin G, proteinase 3, and neutrophil elastase which would be present in Opa+ Gc phagosomes. In order to determine the role of PMN proteases in killing Opa+ Gc, PMNs were incubated with a protease inhibitor cocktail prior to infection with Opa- or Opa+ Gc. Inhibition of PMN proteases significantly increased the number of viable Opa+ Gc recovered from PMNs (Figure 28F) and significantly enhanced the percent of viable Opa+ Gc inside PMNs (Figure 28A-E). These data reinforce our previous observations that PMN proteases have antigonococcal activity, and suggest that Opa+ Gc reside in a mature compartment that contains all of the PMN primary granule protease which contributes to the observed Gc survival defect inside PMNs.

In addition to residence in a mature phagolysosome, intrinsic susceptibility of Opa- or Opa+ Gc could contribute to the observed differences in survival post exposure to PMNs. The human cathelicidin, LL-37 and the protease cathepsin G have been shown to have antigonococcal activity (Rest, 1979, Rock et al., 1988, Shafer et al., 1986b, Shafer, 1988). In order to determine if increased susceptibility of Opa+ Gc to these components contributes to the observed survival defect, Opa- and Opa+ Gc were incubated with increasing concentrations of LL-37 or cathepsin G for $60 \mathrm{~min}$ and viable CFU plated. While exposure to LL-37 and cathepsin G resulted in killing of both Opaand Opa+ Gc, there was no significant difference in their susceptibility to these 
antimicrobial components (Figure 29A and B). We conclude that increased susceptibility of Opa+ Gc to PMN killing is due primarily to residence in mature phagolysosomes, where they are exposed to antimicrobial compounds, rather than increased susceptibility to these components.

\subsubsection{Src-family kinase signaling is required for PMNs to direct Opa+Gc into mature phagolysosomes where they are killed}

Degranulation and induction of the oxidative burst in PMNs involves signaling signaling via Syk kinase, phosphoinositide 3-kinase (PI3K), the small GTPase Rac, and Src-family kinases (Van Ziffle et al., 2009, Fensome et al., 1996, Sengelov et al., 1993, Werner, 2004, Abdel-Latif et al., 2005, Mohn et al., 1995). These regulators are triggered upon engagement of CEACAM3 (Buntru et al., 2012, Sadarangani et al., 2011). Therefore, we hypothesized these four signaling components would contribute to the antimicrobial activity of PMNs against Opa+ Gc.

To test this hypothesis we used piceatannol, LY 294002, EHT 1864, and PP1 to inhibit Syk kinase, phosphoinositide 3-kinase (PI3K), the small GTPase Rac, and Srcfamily kinases, respectively. The inhibitors were used at a concentration that significantly reduced the PMN oxidative burst in response to infection with Opa+ Gc (Figure 31A). Adherent PMNs were treated with each of the inhibitors prior to infection with Opa+ Gc and bacterial survival was evaluated using Baclight viability dyes. None of the inhibitors significantly reduced internalization of Opa+ Gc by adherent, IL-8 primed PMNs (Figure 31B). Only PMN treatment with the Src-family kinase member inhibitor, PP1 significantly increased Opa+ Gc intracellular survival relative to control, untreated cells (Figure 30B-H). Additionally, PMN treatment with PP1 further rescued survival of Opa- 
Gc in PMNs from 51\% to 76\% (Figure 30G-I) and did not affect internalization (Figure 31C). These data suggest an important role for Src-family kinase member signaling in the ability of PMNs to kill Gc, particularly Opa+ Gc.

In order to test the hypothesis that Src-family kinase signaling is required for granule fusion with the Gc phagosome, PMNs were treated with PP1 followed by infection with Opa+ Gc. Phagosome maturation was assessed using antibodies directed against neutrophil elastase. PP1 treatment of PMNs significantly reduced the percent of Opa+ Gc residing in phagosmes positive for neutrophil elastase (Figure 32A and C). In contrast, PMN treatment with piceatannol did not affect Opa+ Gc residence in mature phagosomes. The Src-family kinase member, Hck upon activation has been shown to associate with PMN primary granules and be present on mature phagosomes (Fumagalli et al., 2007, Mocsai et al., 2000, Mohn et al., 1995). Moreover, we observed via immunofluorescence, increased recruitment of the Src-family kinase member, Hck to Opa+ Gc phagosomes in PMNs (Figure 32B and D). From these data we conclude that in adherent, IL-8 primed human PMNs, Src-family kinase signaling, particularly Hck, is required for Opa+ Gc phagosomes to fuse with PMN primary granules and become mature degradative phagolysosomes.

\subsubsection{Activation of Src-family kinase signaling increases PMN killing of Opa-Gc}

Given that Opa expression continually varies in vivo, we sought to determine if the presence of Opa+ Gc affects survival of Opa- Gc in PMNs or vice versa. PMNs were infected individually with either Opa+ or Opa- Gc or coinfected with an equal MOI, and survival of Opa+ and Opa- Gc was assessed. We observed a decrease in Opa- Gc survival during coinfection with Opa+ Gc (Figure 33A). Our results suggest that during 
coinfection, the presence of Opa+ Gc enhances PMN killing of Opa- Gc. We observed that Src-family kinase signaling is important for mediating killing of Opa+ Gc in PMNs. In order to determine if activation of Src-family kinase signaling is sufficient to decrease Opa- Gc survival in PMNs, PMNs were treated with a synthetic activator of Src-family kinase members prior to infection with Opa- Gc. Opa- Gc survival evaluated using Baclight viability dyes. Treatment with the Src-family kinase activator results in decreased survival of Opa- Gc in PMNs (Figure 33B-D). From these results we conclude that Opa+ stimulation of PMNs or direct activation of Src-family kinase signaling is sufficient to increase PMN killing of Opa- Gc.

\subsection{Discussion}

The aim of this study was to investigate mechanisms which lead to the differential survival of Opa+ and Opa- Gc in PMNs. Our current data demonstrate that Opa+ Gc have primarily reduced intracellular survival in PMNs compared to Opa- Gc. We observed that this was CEACAM-dependent because Opa+ Gc survival in PMNs can be rescued when Gc engagement of CEACAMs is prevented. Our results show that PMN killing of Opa+ Gc is due to residence in a mature degradative phagolysosome containing oxidative and nonoxidative components. We observed that preventing formation of a degradative phagolysosome by inhibiting Src-family kinases or by inhibiting PMN protease activity is sufficient to rescue Opa+ Gc intracellular survival. Therefore, our work supports a model in which Opa+ Gc interact with CEACAM receptors on the PMN surface (Figure 34). This interaction results in internalization and also triggers signaling events necessary for both primary and secondary granule fusion with Opa+ Gc phagosomes. As a result of 
phagosome-granule fusion, Opa+ Gc are exposed to an array of non-oxidative antigonococcoal components, leading to their reduced intracellular survival in PMNs.

While PMNs possess both mechanisms to kill bacteria extracellularly and intracellularly, we observed that the majority of Opa+ and Opa- Gc survive extracellularly suggesting extracellular mechanisms of killing are generally not as effective against Gc. Additionally, we observed that Opa+ Gc have primarily an intracellular survival defect in PMNs. These data further emphasize the importance of PMN intracellular killing mechanisms against Gc.

PMNs kill bacteria intracellularly by directing antimicrobial granule fusion with bacteria containing phagosomes (Borregaard, 2010). Our data indicate that in contrast to Opa- Gc phagosomes, Opa+ Gc phagosomes fuse with both secondary and primary granules. Therefore, Opa+ Gc are exposed to oxidative and non-oxidative antimicrobial components. PMN secondary granules contain subunits of the NADPH oxidase complex, gp91 $1^{\text {phox }}$ and $\mathrm{p} 22^{\text {phox }}$ which are necessary for generation of the oxidative burst. Activation of the NADPH oxidase complex generates superoxide, which can dismutate to hydrogen peroxide. Myeloperoxidase delivered from primary granules then converts hydrogen peroxide to hypochlorous acid (Borregaard et al., 2007, Borregaard, 2010). Our results demonstrate that even though Opa+ Gc stimulates a potent PMN oxidative burst, there is no significant difference in Opa+ Gc survival in PMNs treated with the NADPH oxidase inhibitor, DPI. This finding is in agreement with several studies demonstrating that PMN killing of Gc is primarily via non-oxidative granule components (Seib et al., 2005, Criss et al., 2009, Criss et al., 2008, Rest et al., 1982, Wu et al., 2009). Gc possess many 
mechanisms for detecting and defending against damage induced by ROS (Johnson et al., 2011). Gc catalase activity is essential for defense against ROS in vitro and in the mouse model a Gc catalase mutant is attenuated. However, the mutants are still attenuated in mice which are unable to generate a PMN oxidative burst (Wu et al., 2009). These data suggest that Gc defenses against ROS are not required for protection against PMN generated ROS but perhaps are necessary to defend against ROS from other sources in vivo.

In the phagosome, Opa+ Gc would be exposed to the secondary and primary granule non-oxidative components lysozyme, LL-37, $\alpha$-defensins, cathepsin G, BPI, neutrophil elastase, and proteinase 3(Borregaard et al., 2007). Of these components LL37, cathepsin G, and BPI have antigonococcal activity (Shafer et al., 1998, Shafer et al., 1986b, Casey et al., 1985). Our data demonstrate that in the context of the PMN phagosome, protease activity is particularly important for mediating killing of Opa+ Gc, as inhibition of protease activity rescues intracellular survival. Since Opa+ Gc and OpaGc display similar susceptibility to both purified LL-37 and cathepsin G in vitro, our data supports a model in which Gc residence or avoidance of a degradative phagolysosome is a key factor in determining Gc survival in PMNs.

Our results suggest that the Opa+ Gc survival defect in PMNs is dependent on CEACAM engagement. This aligns with previous data demonstrating CEACAM engagement results in Gc internalization and activation of PMNs. In particular engagement of CEACAM3 has been shown to stimulate PMN antimicrobial activities including the oxidative burst and granule exocytosis (Sarantis et al., 2007). While 
CEACAM1 and CEACAM6 signaling do not result in substantial PMN activation, binding to CEACAM1 or CEACAM6 results in cooperative signaling via CEACAM3 which is sufficient to stimulate PMN antimicrobial processes (Sarantis et al., 2012). Therefore, Opa engagement of any of the CEACAMs on the PMN surface may result in activation of antimicrobial activities which result in killing of Opa+ Gc.

Gonococcal Opa proteins mediate nonopsonic internalization by PMNs (Belland et al., 1992, Virji et al., 1986, Fischer et al., 1988, King et al., 1978b, Gray-Owen et al., 1997a). Gc internalization by PMNs can be blocked by outer membrane vesicles prepared from Opa+ Gc but not Opa- Gc or Opa specific antibodies. This suggests that Opa protein engagement of CEACAM is required for internalization (Shafer et al., 1989, Elkins et al., 1990). In contrast, we observed that in the absence of CEACAM engagement or CEACAM induced signaling, Opa+ Gc is still internalized by adherent, IL-8 primed PMNs. This is in agreement with our previously observation that Opa- Gc are internalized by adherent, IL-8 primed PMNs (Johnson et al., 2013b, Criss et al., 2009, Ball et al., 2013, Smirnov et al., 2013). One possible explanation for this discrepancy is differences in PMN activation due to PMN adherence and priming state. Studies are currently ongoing in the lab to identify and characterize this alternative mechanism of internalization.

Opa interaction with CEACAM receptors is known to induce signaling via Syk, PI3K, Rac GTPase, and Src-family kinases, which are necessary for the regulation of PMN granule fusion with target membranes (Sadarangani et al., 2011, Lacy, 2006). We observed that in our system Syk, PI3K, and Rac GTPase signaling are not required for 
intracellular killing of Opa+ Gc by PMNs. This is in contrast to previous reports which showed Syk is required for activation of PMN antimicrobial processes and killing of Gc (Sarantis et al., 2007). We can envision at least three reasons for this discrepancy. First, in our experimental system using adherent, IL-8 primed PMNs may bypass or negate the requirement for Syk signaling. Second, previous studies examined the requirement of Syk signaling for PMN exocytosis. Syk may therefore be required for PMN granule exocytosis but not for granule fusion with phagosomes. Third, previous studies employed recombinant Escherichia coli expressing Opa proteins to determine if Syk signaling was required for PMN bactericidal activity. Therefore, bacterial differences which affect interactions with PMNs and/or PMN activation may account for the observed differences in the requirement for Syk signaling in mediating PMN killing of bacteria.

Our data show that Opa+ Gc residence in a degradative phagolysosome is dependent on Src-family kinase signaling. Receptor stimulation of PMNs leads to activation of a variety of kinases, including the Src-family of nonreceptor tyrosine kinases (Lacy, 2006). Hck, Fgr, and Lyn are expressed in PMNs and associated with specific granule populations. Following activation, Hck associates with the primary granule population and Fgr with the secondary granule population (Mohn et al., 1995, Mocsai et al., 2000). Activation results in Hck localization to mature phagosomes (Fumagalli et al., 2007, Mocsai et al., 2000, Mohn et al., 1995). Pathogenic mycobacteria, have been shown to avoid phagosome-primary granule fusion in PMNs in order to survive intracellularly. Accompanying the reduction in primary granule fusion, researchers observed reduced activation and association of Hck with the phagosome membrane (N'Diaye et al., 1998, Astarie-Dequeker et al., 2000, Cougoule et al., 2002). 
Similar to these observations we observed that Opa+ Gc phagosomes which fuse with primary granules display recruitment of Hck, which is significantly reduced compared with phagosomes containing Opa- Gc. Therefore, we conclude Src-family kinase signaling downstream of Opa+ Gc ligation of CEACAM receptors is particularly important for directing fusion of Opa+ Gc phagosomes with primary granules.

In order to make unambiguous observations about survival of Opa- and Opa+ Gc, we used Opa- Gc in which all 11 opa genes are deleted or Opa+ Gc which are locked for expression of OpaD. However, Gc has the ability to phase vary Opa protein expression on its surface, and as a result during infection there is a mixed population of Gc which lack Opa proteins or express one or multiple Opa proteins (Stern et al., 1986, Murphy et al., 1989). We observed that coinfection of PMNs with Opa- and Opa+ Gc resulted in reduced survival of Opa- Gc, suggesting during gonorrheal infection the ratio of Opa- and Opa+ Gc may affect Gc survival in PMNs.

Selection for Opa+ Gc occurs in naturally and experimentally infected individuals and in the female mouse model (James et al., 1978, Hobbs et al., 2011, Jerse, 1999, Cole et al., 2010). However, Opa+ Gc are more readily killed by PMNs (Criss et al., 2009, Ball et al., 2013, Rest et al., 1982, Virji et al., 1986). Therefore, Opa protein expression would be both advantageous and disadvantageous during gonorrheal infection. Two advantages previously reported are Opa protein expression mediates colonization of epithelial cells and Opa proteins provide resistance to proteases found in cervical secretions (Kupsch et al., 1993, Soderholm et al., 2011, Wang et al., 1998, Makino et al., 1991, Simms et al., 2006, Jerse, 1999). The data presented here indicate one major 
disadvantage is Opa+ Gc stimulate PMN antimicrobial responses leading to residence in a degradative phagolysosomes and decreased survival. Therefore, in the context of PMNs, Opa- Gc have a survival advantage. Despite increased killing of Opa+ Gc by PMNs, Opa protein mediated stimulation of PMNs may serve as an advantage by facilitating Gc pathogenesis in two ways. First, the PMN oxidative burst and degranulation results in tissue damage which may increase bacterial transmigration across epithelial cells allowing for dissemination (Zen et al., 2003). Second, recruitment of PMNs to the site of infection may result in internalization of Opa- Gc which remain viable inside PMNs. PMNs may then transport Opa- Gc across the epithelial cell barrier or to new individuals allowing for increased dissemination and transmission, respectively. In support of this hypothesis, disseminated Gc recovered from the fallopian tubes of women with salpingitis were reported to be Opa-(Draper et al., 1980). From our current knowledge in the field we propose that a balance of Opa+ Gc and Opa- Gc as well as the ability of Gc to vary expression of Opa proteins would be critical for gonorrheal disease persistence and progression. 


\subsection{Figures for Chapter 3}

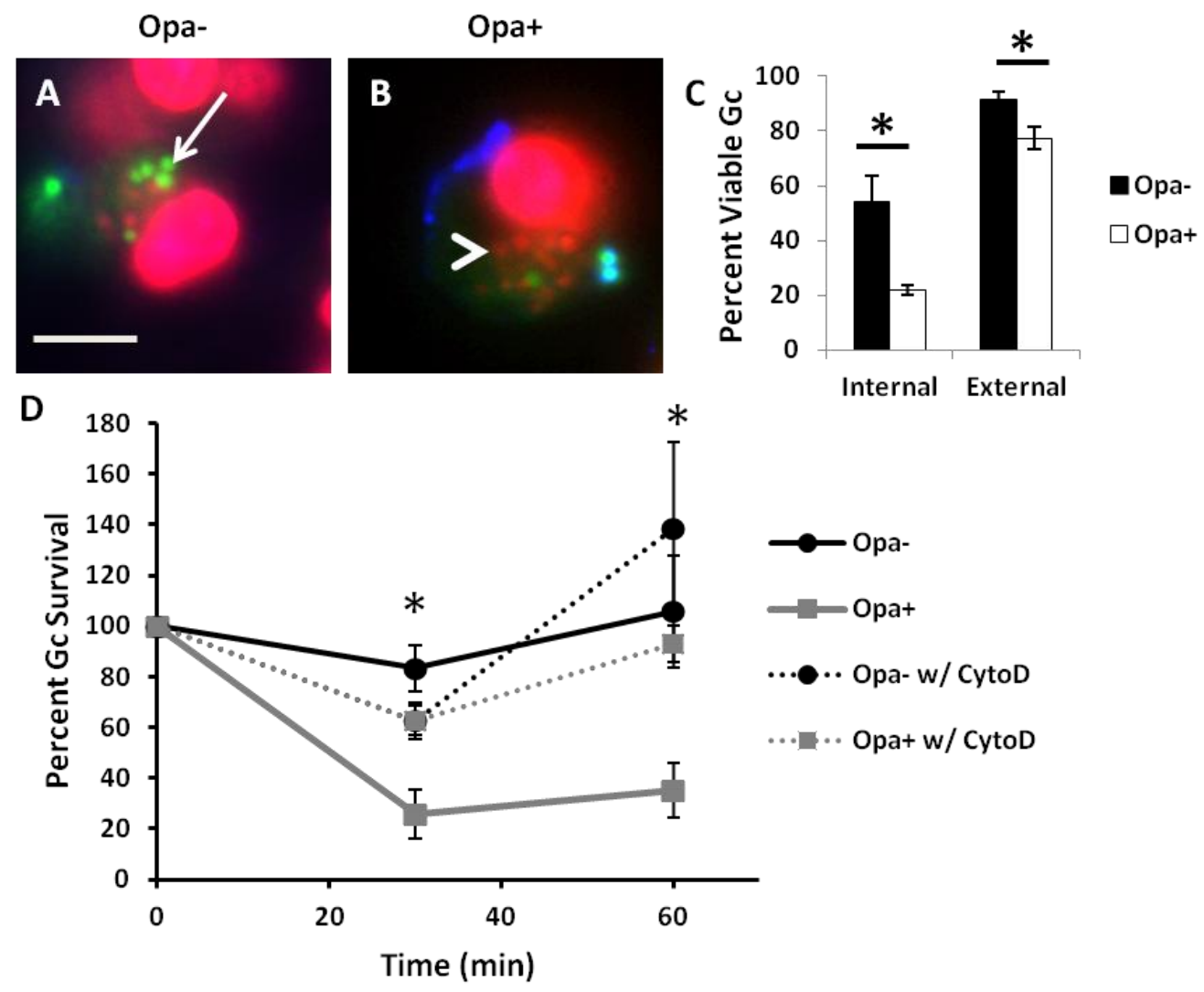

Figure 23: Opa+ Gc have reduced survival after exposure to PMNs.

PMNs were infected with Opa- (A) or Opa+ (B) Gc for 1hr. Viable Gc (green) and nonviable Gc (red) were discriminated using Baclight viability dyes SYTO9 and propidium iodide, and extracellular Gc were labeled with soybean lectin (blue). Extracellular viable Gc appear teal, intracellular nonviable Gc appear red, and intracellular viable Gc appear green. Arrows indicate viable, intracellular Gc and arrow heads indicate nonviable, intracellular Gc. Scale bar equals $5 \mu \mathrm{m}$. The percent of viable extracellular and intracellular Gc are reported in C. Asterisks indicate $P<0.05$ by Student's two-tailed $t$ test. D. Opa- and Opa+ Gc were exposed to PMNs that were left untreated or treated with cytochalasin D (CytoD). Bacterial survival was calculated as CFU enumerated from PMN lysates at each time point, divided by the CFU present at 0 min. Asterisks indicate $P<0.05$ by Student's two-tailed $t$ test. 

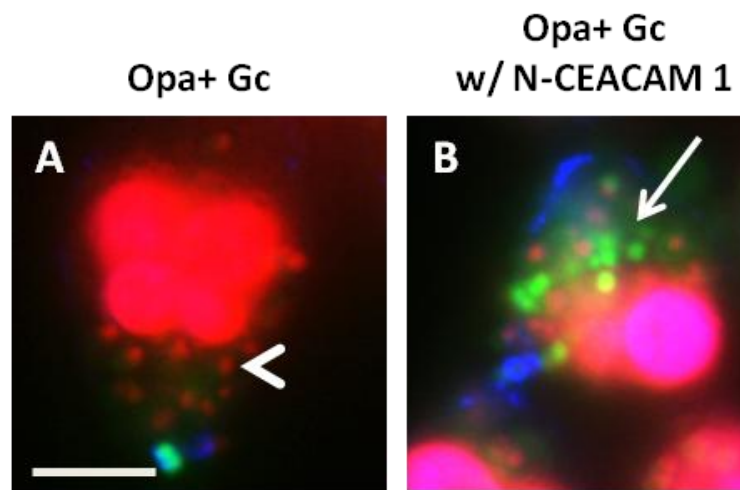

Opa+ Gc w/ N-CEACAM 1

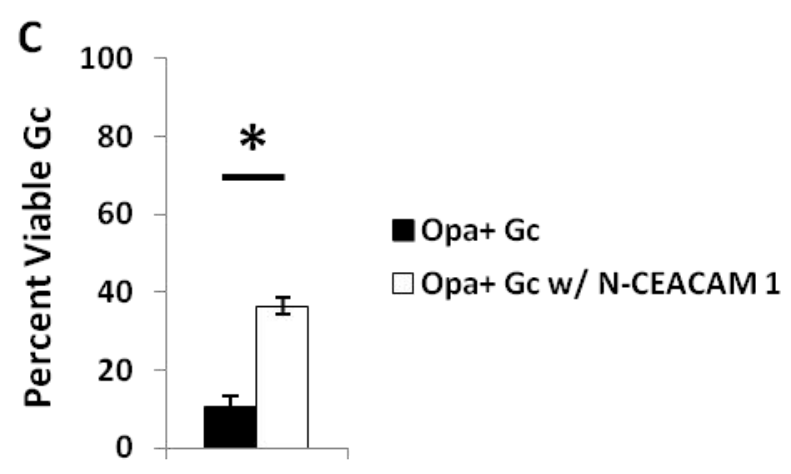

Figure 24: PMN killing of Opa+ Gc is dependent on engagement of CEACAMs.

PMNs were infected with Opa+ (A) or Opa+ Gc coated with the $\mathrm{N}$ domain of CEACAM 1 (B). Viability of individual Gc was assessed as in Figure 23A-B. Arrows indicate viable, intracellular Gc and arrow heads indicate nonviable, intracellular Gc. Scale bar equals $5 \mu \mathrm{m}$. The percent of viable intracellular Gc are reported in C. Asterisks indicate $P$ $<0.05$ by Student's two-tailed $t$ test. 


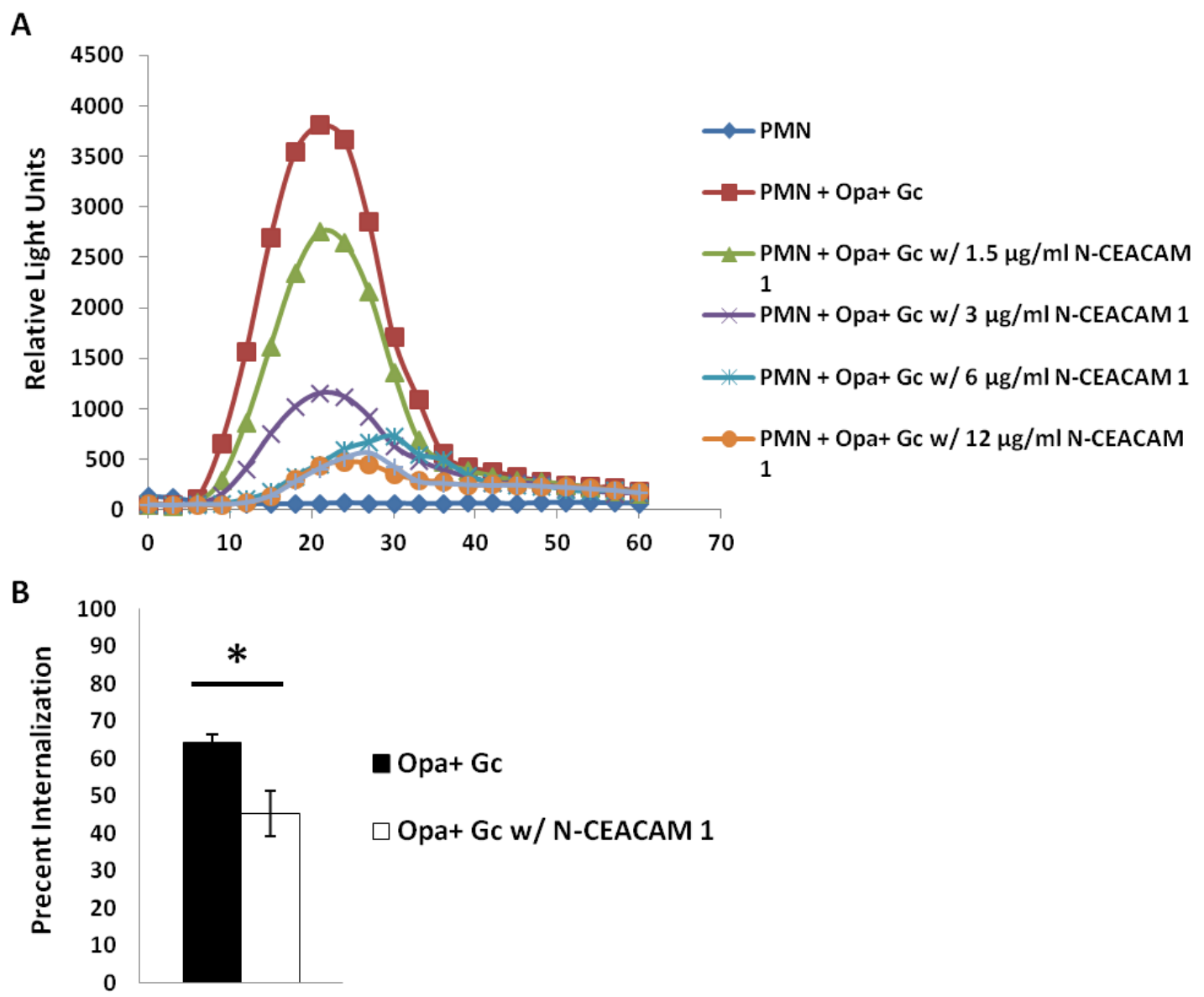

Figure 25: Effect of coating Opa+ Gc with N-CEACAM1 on the PMN oxidative burst and Gc internalization.

A. Prior to infection of PMNs, Opa+ Gc was coated with indicated concentrations of NCEACAM or left uncoated. ROS production was measured as luminol-dependent chemiluminescence produced over $1 \mathrm{hr}$. B. PMNs were infected with Opa+ Gc or Opa+ Gc with N-CEACAM1 for $1 \mathrm{hr}$. To determine percent internalization intracellular and extracellular Gc were discriminated using immunofluorescence. 

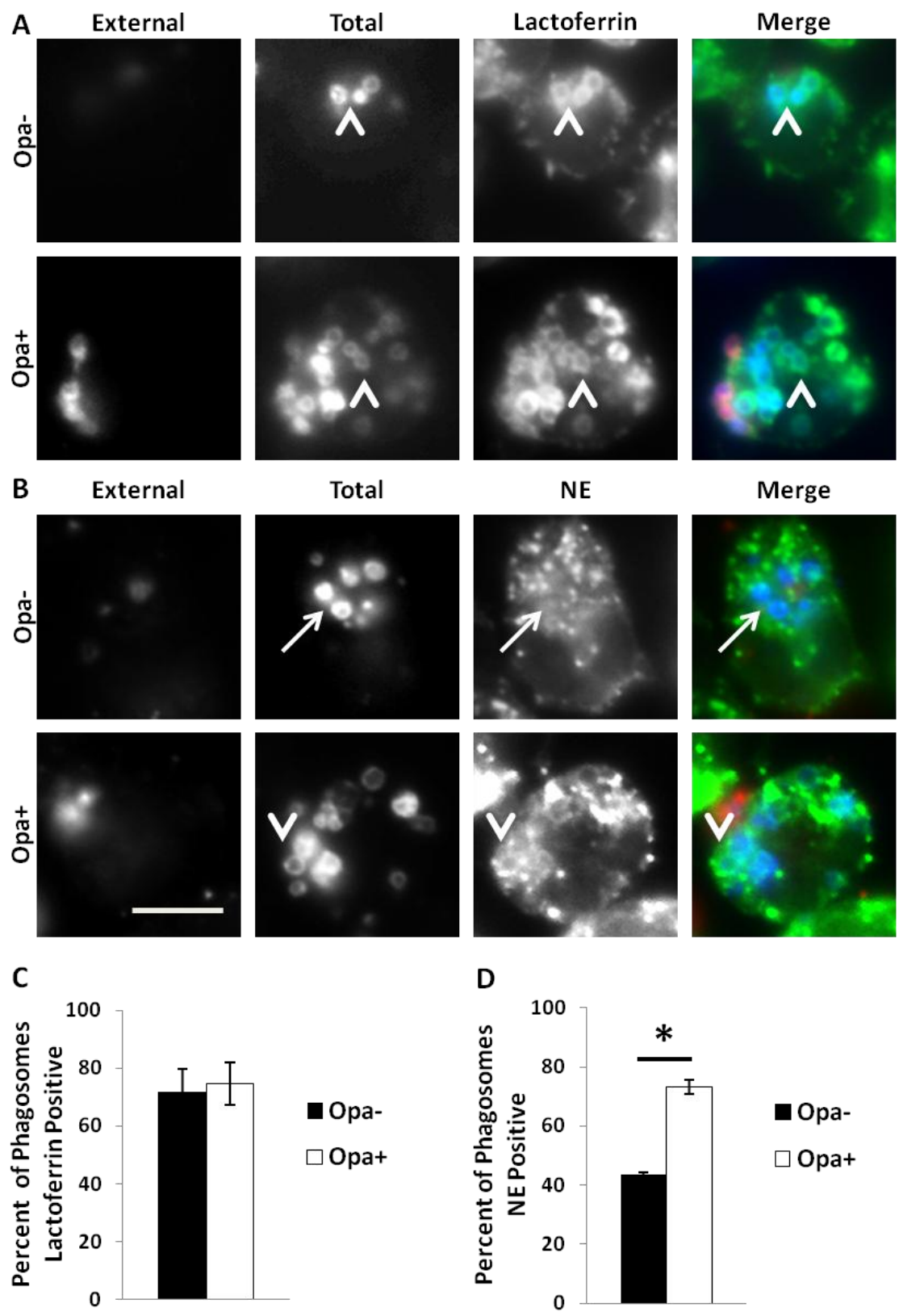

Figure 26: Phagosomes containing Opa+ Gc are significantly more mature than those containing Opa- Gc. 
PMNs were infected with Opa- or Opa+ Gc for $1 \mathrm{hr}$. External Gc appear red/blue and internal Gc appear blue. PMNs were stained with antibodies against the secondary granule protein lactoferrin (A) or the primary granule protein neutrophil elastase (NE) (B), which appear green. Arrowheads indicate bacterial phagosomes positive for granule proteins, while arrows indicate phagosomes negative for granule proteins. Scale bar equal $5 \mu \mathrm{m}$. The percent of Opa + and Opa- Gc phagosomes positive for lactoferrin or neutrophil elastase are reported in $\mathrm{C}$ and $\mathrm{D}$, respectively. Asterisks indicate $P<0.05$ by Student's two-tailed $t$ test. 


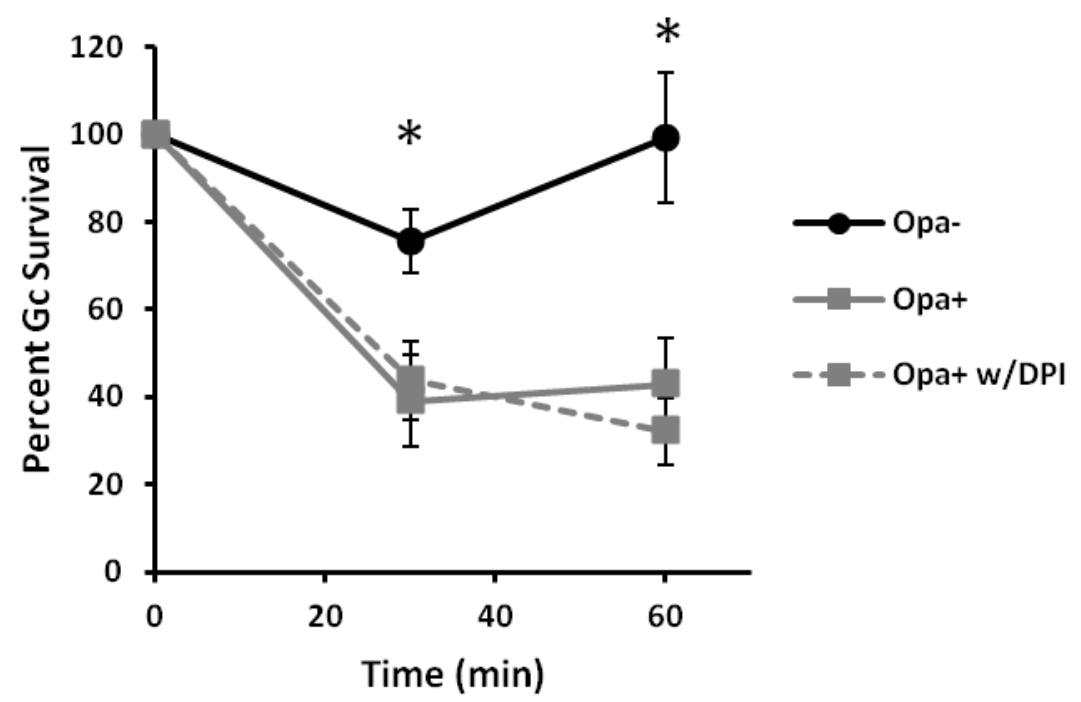

Figure 27: Increased killing of OpaD+ Gc by PMNs is primarily non-oxidative.

PMNs were pretreated with $10 \mu \mathrm{M}$ DPI and infected with Opa- or Opa+ Gc. Viable Gc were enumerated as described in Figure 23D. Asterisks indicate $P<0.05$ for Opa+ Gc vs Opa- Gc by Student's two tailed $t$ test. 


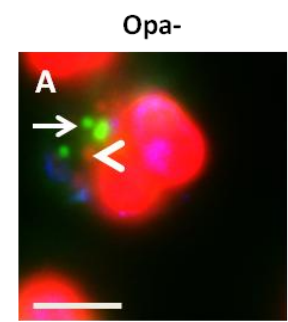

Opa+
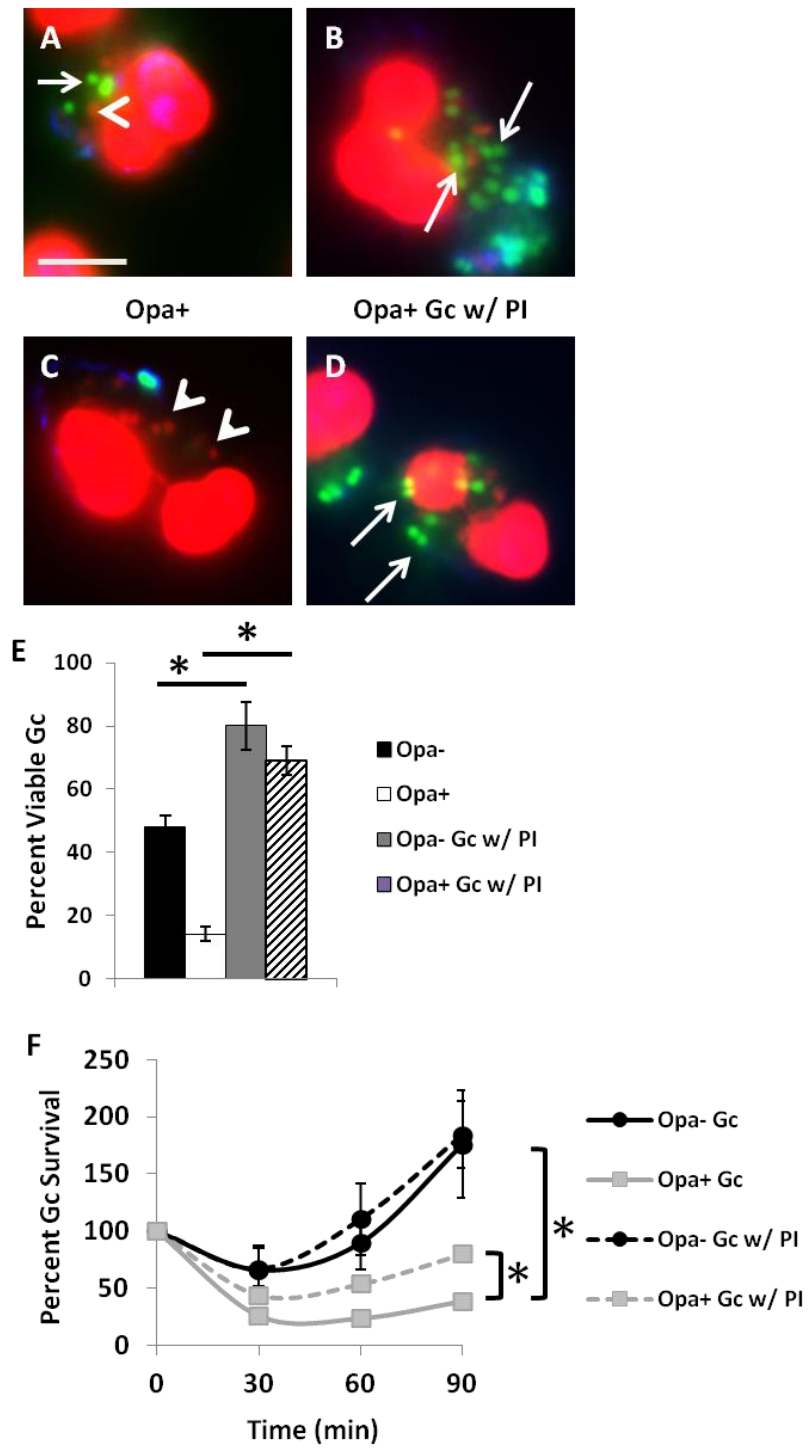

$\mathrm{Opa}+\mathrm{Gc}$ w/ PI

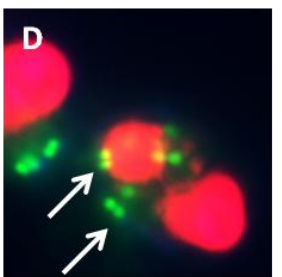$$
\text { P }
$$ 
A
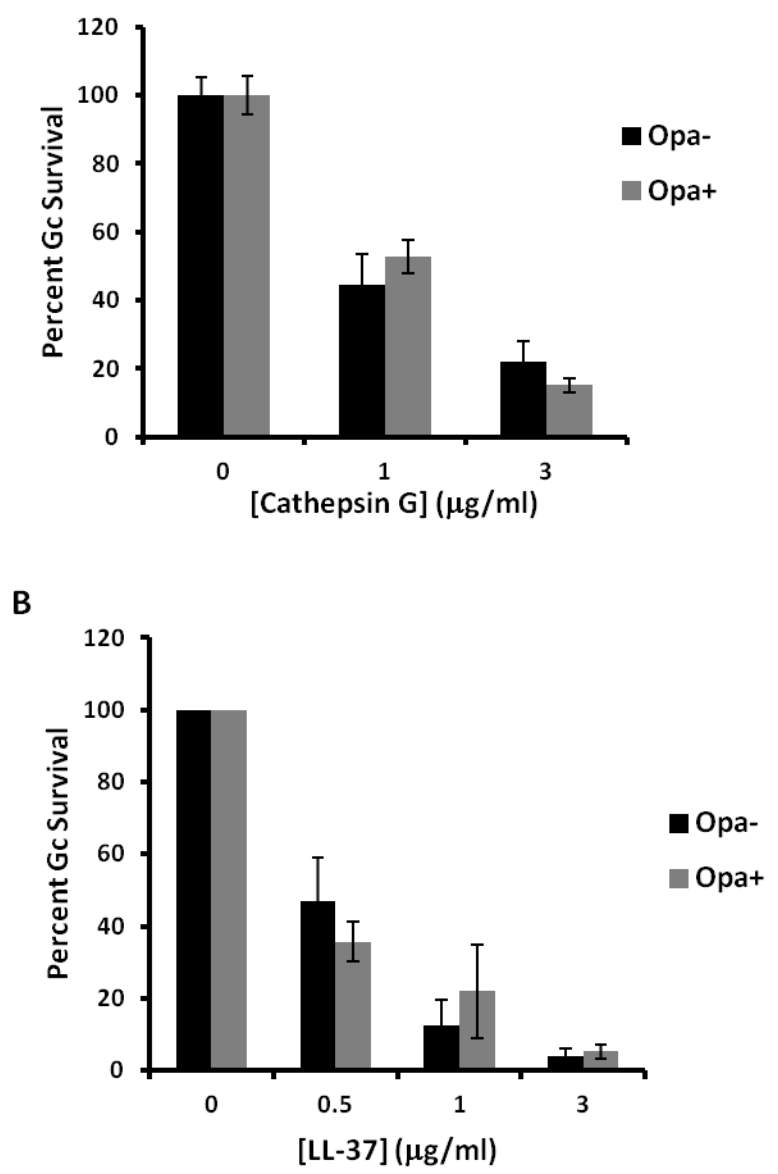

Figure 29: Expression of Opa proteins by Gc does not enhance bacterial sensitivity to antimicrobial peptides.

Gc were incubated with cathepsin-G (A) or LL-37 (B) for $45 \mathrm{~min}$. While survival of each strain was significantly reduced at each concentration of protein relative to untreated $\mathrm{Gc}$, there was no statistically significant difference in Opa+ vs Opa- Gc sensitivity at each concentration. 


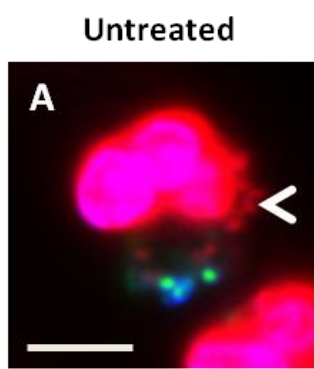

EHT 1864

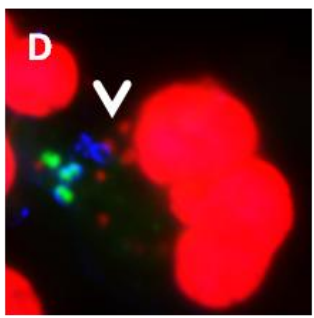

F

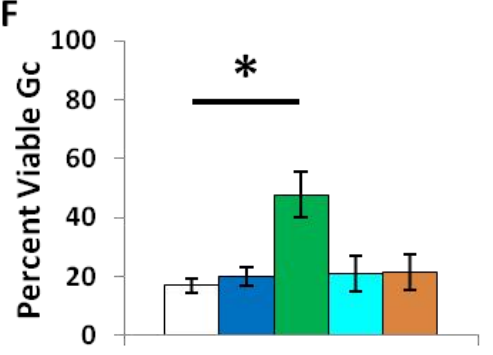

Untreated
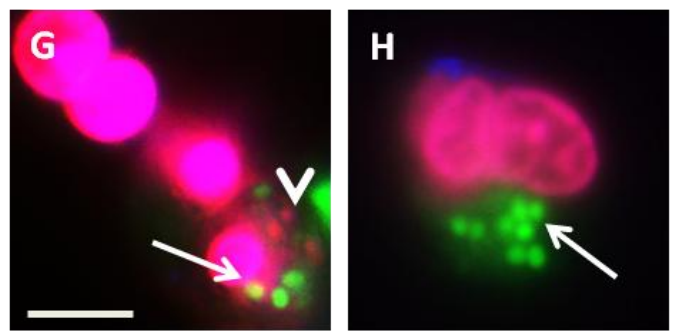

I

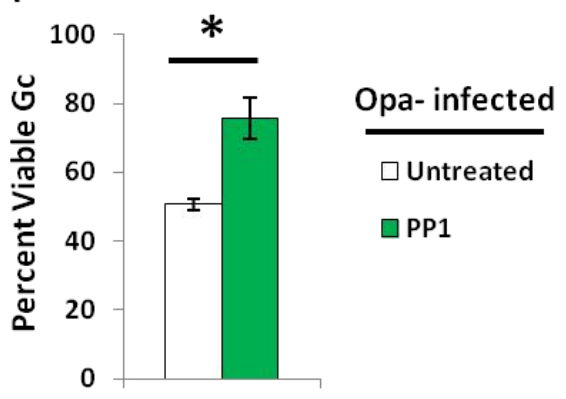

PP1

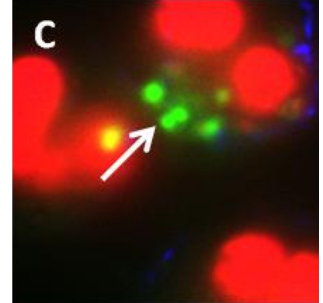

LY 294002

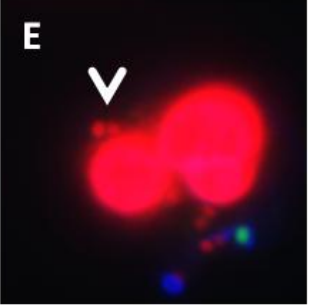

Opa+ infected

$\square$ Untreated

$\square$ Piceatannol

$\square$ PP1

口 ЕHT1864

$\square$ LY294002

Figure 30: Inhibition of Src-family kinase signaling increases Gc survival in PMNs. 
Untreated PMNs (A), PMNs treated with piceatannol (B), PP1 (C), EHT1864 (D), or LY 294002 (E) were infected with Opa+ Gc for 1 hr. Untreated (G) or PMNs treated with PP1 $(\mathrm{H})$ were infected with Opa- Gc for $1 \mathrm{hr}$. The viability of individual bacteria was assessed as in Figure23A-B. Arrows indicate viable, intracellular Gc and arrowheads indicate nonviable, intracellular Gc. Scale bar equal $5 \mu \mathrm{m}$. The percent of viable intracellular Gc are reported in F and I. Asterisks indicate $P<0.05$ by Student's twotailed $t$ test. 

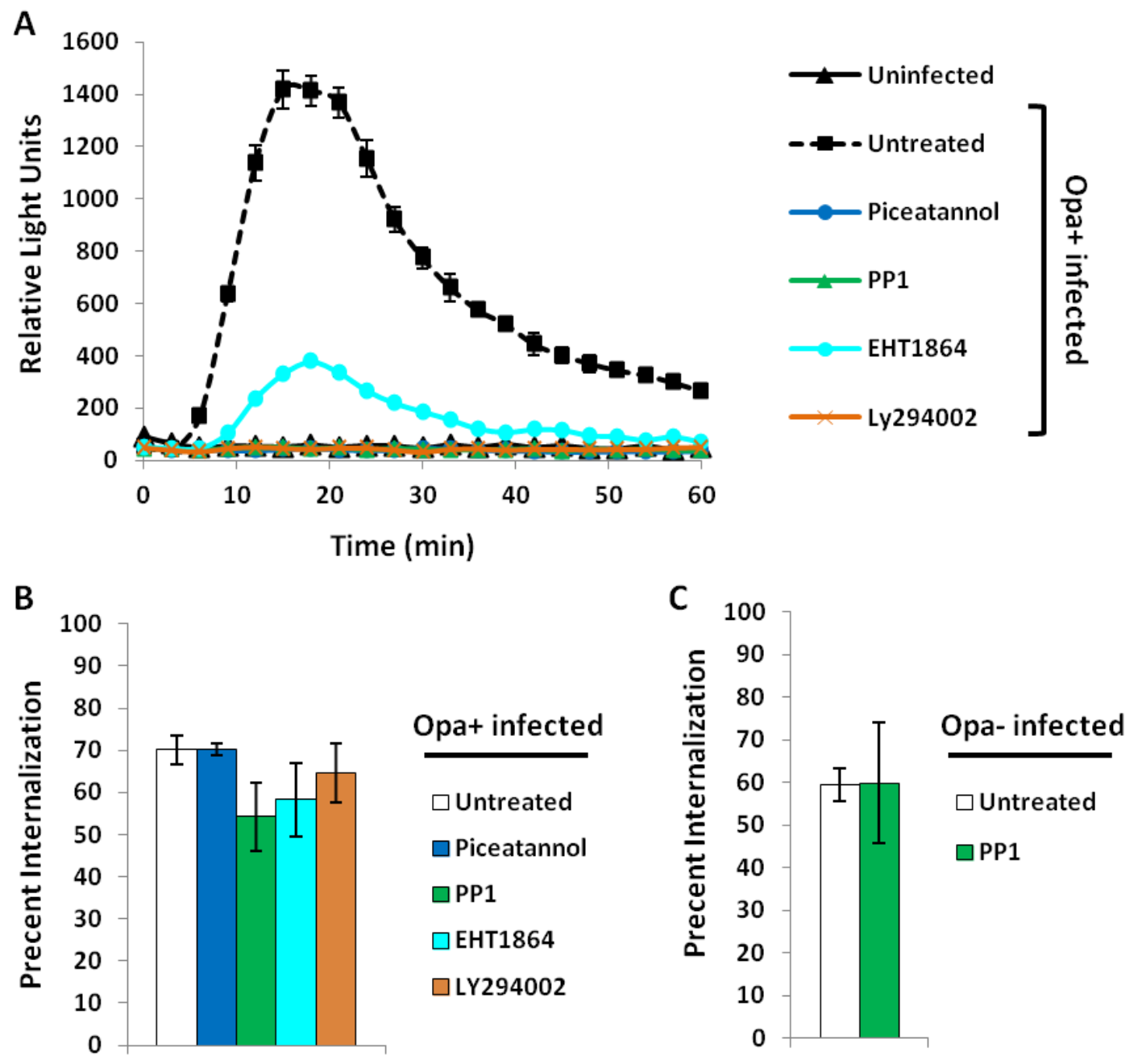

Figure 31: Effect of CEACAM signaling inhibitors on the PMN oxidative burst and Gc internalization.

A. PMNs were treated with piceatannol, LY 294002, EHT1864, or PP1 or left untreated, then infected with Opa+ Gc. ROS production was measured as luminol-dependent chemiluminescence produced over $1 \mathrm{hr}$.B. PMNs were treated with piceatannol, LY 294002, EHT1864, or PP1 or left untreated, then infected with Opa+ Gc for 1hr. C. PMNs were treated with PP1 or left untreated, then infected with Opa- Gc for 1hr. To determine percent internalization intracellular and extracellular Gc were discriminated using immunofluorescence. 

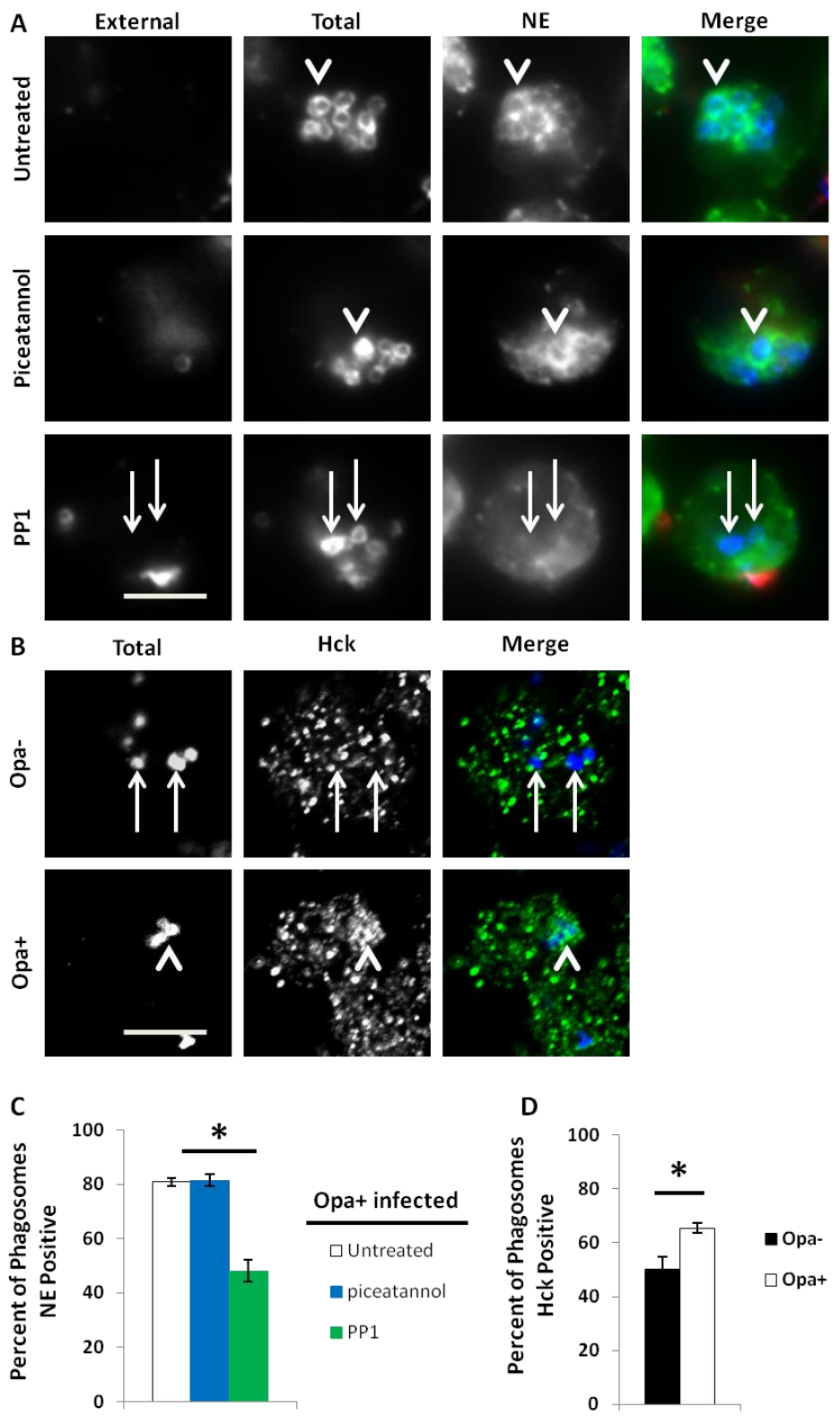

Figure 32: Src-family kinase activity is crucial for Opa+ Gc phagosome fusion with primary granules 
PMNs treated with piceatannol or PP1 or were left untreated, then infected with Opa+ Gc for $30 \mathrm{~min}$. Immunofluorescence was conducted to discriminate external Gc (red/blue) and internal Gc (blue). PMNs were stained with antibodies against the primary granule protein neutrophil elastase (NE) (A), which appears green. B. PMNs were infected with CFSE labeled Opa- or Opa+ Gc for $1 \mathrm{hr}$. PMNs were stained with an antibody against the Src-family kinase member, Hck, which appears red. Arrowheads indicate bacterial phagosomes positive for granule proteins or Hck, while arrows indicate phagosomes negative for granule proteins or Hck. Scale bar equals $5 \mu \mathrm{m}$. The percent of phagosomes positive for neutrophil elastase and Hck is reported in C and D, respectively. Asterisks indicate $P<0.05$ by Student's two-tailed $t$ test. 

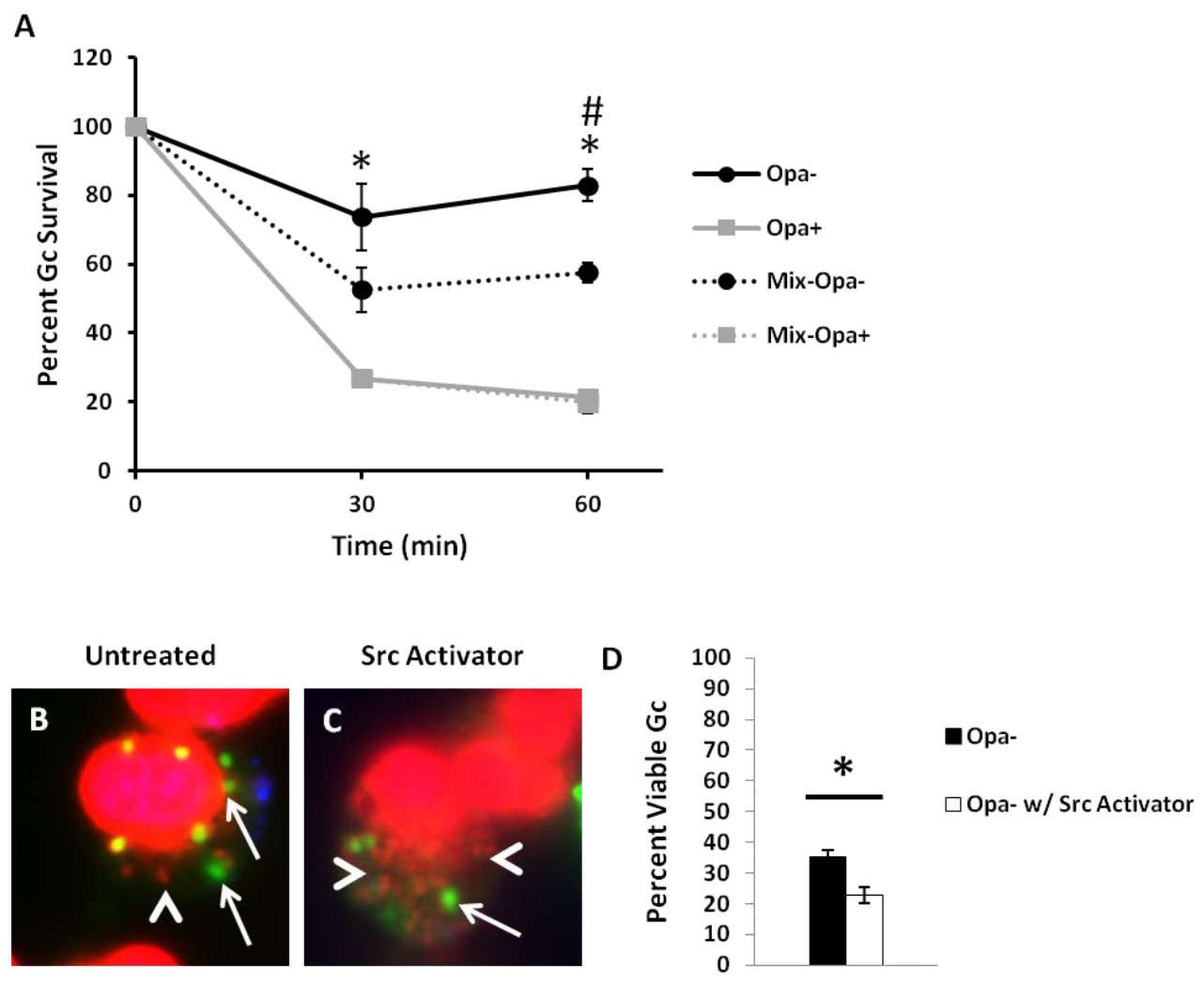

Figure 33: Activation of Src-family kinase activity decreases Opa- Gc survival in PMNs.

A. PMNs were infected with Opa-, Opa+ Gc, or a 1:1 mix of Opa- and Opa+ Gc. Viable Gc was enumerated as described above. Opa- and Opa+ Gc were differentiated by eye under light microscopy. Mix Opa- and Mix Opa+ Gc represent the percentage change in opa- and opaque colonies measured in the coinfection viable Gc, compared with their numbers measured at $0 \mathrm{~min}$. Asterisks indicate $P<0.01$ by Student's two tailed $t$ test between Opa- and Opa+ Gc at 30 and $60 \mathrm{~min}$. Hash indicates $P<0.01$ by Student's two tailed $t$ test between Opa- and Mix Opa- Gc at 60 min. Untreated PMNs (B) and PMNs treated with a Src-family kinase activator (C) were infected with Opa+ Gc for $1 \mathrm{hr}$. The viability of individual bacteria was assessed as in Figure1A-B. Arrows indicate viable, intracellular Gc and arrowheads indicate nonviable, intracellular Gc. Scale bar equal $5 \mu \mathrm{m}$. The percent of viable intracellular Gc are reported in D. Asterisks indicate $P<0.05$ by Student's two-tailed $t$ test. 


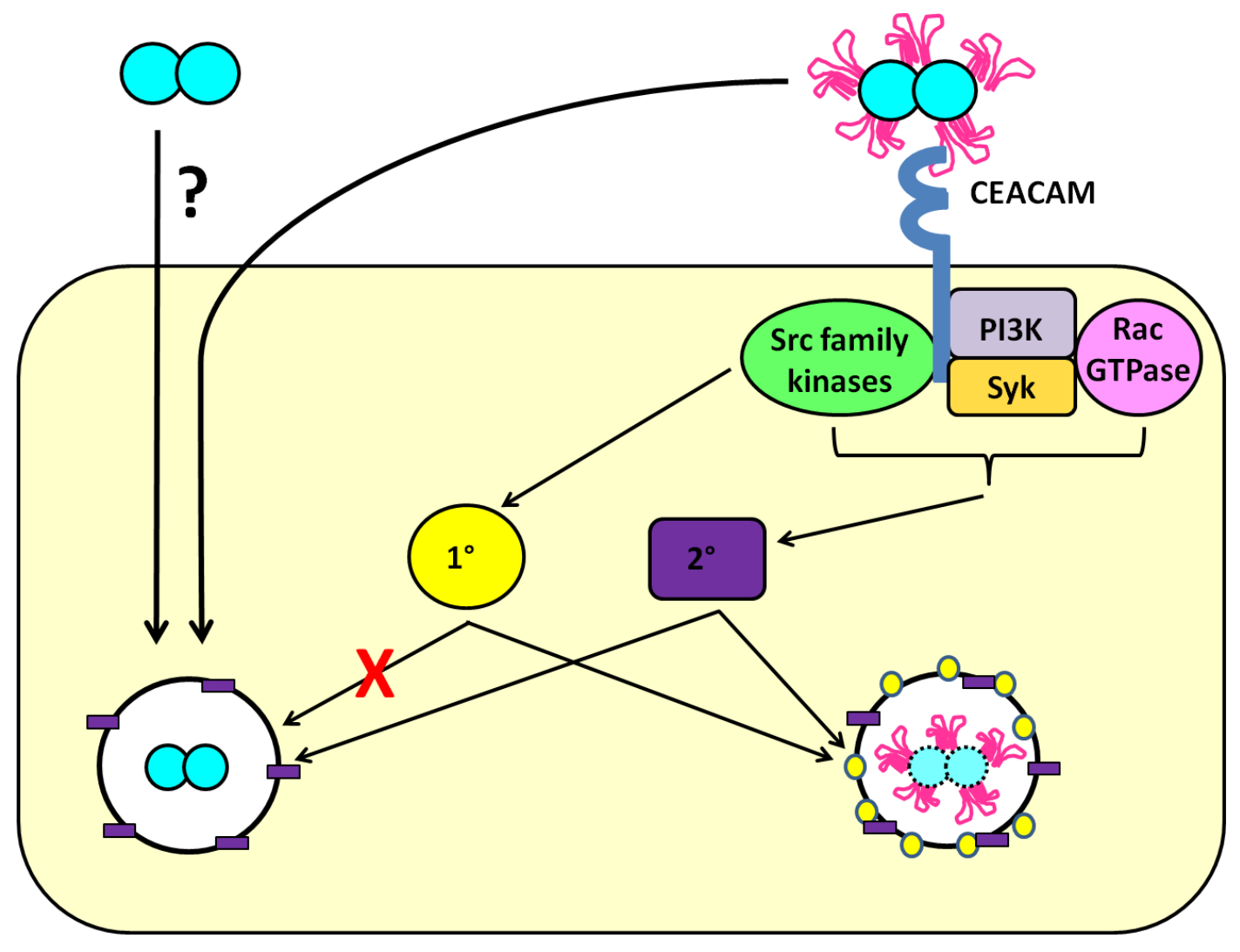

Figure 34: Model for survival of Opa- and Opa+ Gc in PMNs 


\section{Chapter 4: Discussion and Future Directions}

\subsection{Summary}

This dissertation research was undertaken with the knowledge that viable Gc can be cultured from gonorrheal patient exudates containing abundant PMNs, and that 50\% of internalized Gc remain viable inside primary human PMNs infected ex vivo. (Wiesner et al., 1980, Criss et al., 2009, Simons et al., 2005). However, how Gc survives inside PMNs was still an open question in the field. Prior to my studies, it was assumed that Gc survived inside PMNs by using mechanisms to intrinsically resist killing by oxidative and non-oxidative PMN components (Criss et al., 2012). In order to defend against oxidative damage Gc has a superoxide dismutase, a catalase, and several peroxidases to detoxify ROS (Soler-Garcia et al., 2004, Wilks et al., 1998, Seib et al., 2004); and a methionine sulfoxide reductase and RecN to repair oxidative damage to proteins and DNA, respectively (Seib et al., 2004, Skaar et al., 2002, Stohl et al., 2006). In order to defend against non-oxidative antimicrobial components, Gc modifies its surface by addition of phosphoethanolamine (PEA) and sialylation of lipooligosaccharide (LOS) (Wu et al., 2006a, Lewis et al., 2009, Gilbert et al., 1996) and exports antimicrobial components using the Mtr and FarAB efflux pumps (Shafer et al., 1998, Lee et al., 1999). The putative metalloprotease Ngo1686, which has the ability to cleave peptidoglycan and affect pilus biogenesis, can additionally protect against both oxidative and non-oxidative components (Stohl et al., 2013).

My studies demonstrate a complementary, novel mechanism of Gc intracellular survival in PMNs. In gonorrheal exudates and human PMNs infected ex vivo, we observed that Gc phagosomes delay fusion with primary granules to avoid maturation 
into degradative phagolysosomes. Viable Gc were more likely to reside in immature phagosomes, demonstrating a correlation between phagosome maturity and Gc survival. Active Gc processes were not required for residence in immature phagosomes, suggesting Gc surface characteristics determine phagosome maturity. In support of this hypothesis, immunoglobulin opsonization of Gc and expression of Opa proteins was sufficient to overcome delayed phagosome-primary granule fusion. Increasing phagosome-primary granule fusion via immunoglobulin opsonization of Gc or Opa protein expression resulted in decreased intracellular Gc viability due in part to serine protease activity. Further exploration of the survival defect observed for Opa+ Gc demonstrated that CEACAM signaling via Src-family kinase members stimulates primary granule fusion with Gc phagosomes, which results in killing of Opa+ Gc. We conclude from these studies that one method for Gc to avoid PMN clearance in acute gonorrhea is by delaying primary granule-phagosome fusion, which is influenced by Gc's surface characteristics, thus preventing formation of a degradative phagolysosome.

Several essential questions arise from our initial characterization of Gc survival inside PMNs, which will be discussed below (Figure 35). First, what is the mechanism of Opa- Gc internalization by PMNs? Second, what are the PMN components that have antigonococcal activity? Third, are Gc capable of replicating inside PMNs? Finally, how do expression and phase variation of Opa proteins affect Gc survival in PMNs?

\subsection{Route of Internalization of Opa- Gc}

Our results demonstrate that in contrast to Opa+ Gc, Opa- Gc are internalized by PMNs and reside in an immature phagosome. Prior to our work, there was substantial evidence indicating that internalization of unopsonized Gc requires expression of Opa 
proteins that engage CEACAMs on the PMN surface (Elkins et al., 1990, Belland et al., 1992, Virji et al., 1986, Fischer et al., 1988, King et al., 1978b, Gray-Owen et al., 1997a). However, our lab has demonstrated that Opa- Gc are still internalized by adherent, IL-8 primed PMNs (Criss et al., 2009, Ball et al., 2013, Smirnov et al., 2013, Johnson et al., 2013b). In order to fully understand how Gc survive intracellularly in PMNs, it is necessary to characterize the mechanism of Opa- Gc internalization.

Unopsonized, Opa- Gc could be internalized by PMNs via several routes. First, Gc may engage CR3 on PMNs in an opsonin-independent manner to mediate internalization. In cervical epithelial cells, Gc use type IV pili and porins to cooperatively bind CR3 (Edwards et al., 2002b, Edwards et al., 2005, Edwards et al., 2001). Our data indicate that both nonopsonized, Opa- Gc and complement-opsonized, Opa- Gc reside in immature phagosomes in PMNs (Chapter 2 Figure 11) (Johnson et al., 2013b). These results are in agreement with a model in which both complement-opsonized and nonopsonized, Opa- Gc are internalized via CR3 on PMNs and reside in an immature phagosome. However, this route of entry has not been fully investigated in PMNs. In order to address this hypothesis, future experiments could determine if CR3 is required for uptake of Opa- Gc in PMNs by using antibodies against the alpha and beta subunits of CR3 to block CR3-dependent uptake. Additionally, the small GTPase RhoA is required for internalization via CR3 receptors but not Fc receptors (Caron et al., 1998). Therefore, inhibitors of RhoA could also be used to block CR3 mediated phagocytosis. Next, in order to examine the requirement of pili and porin for nonopsonic CR3 dependent internalization, antibodies directed against pili and porin could be used to block Gc interactions with CR3 or internalization could be compared for piliated and nonpiliated 
Opa- Gc. Conducting these experiments will determine if nonopsonized, Opa- Gc internalization by PMNs is via cooperative interaction of pili and porin with CR3. However, our data indicate that nonpiliated Opa- Gc are still internalized by adherent, IL8 primed PMNs (Appendix C), suggesting there are additional pilus-independent mechanisms of Gc internalization by PMNs.

As a second possibility to explain how Opa- Gc is internalized by PMNs, Gc may interact with a currently unknown PMN receptor to mediate internalization. In order to characterize Opa- Gc internalization via an unknown receptor, inhibitors can be used to first differentiate between clathrin and caveolin/lipid raft mediated endocytosis. Further exploration of the unknown receptor can be investigated by examining the surface composition of PMNs. Our data indicate that, in contrast to suspension PMNs and HL-60 cells differentiated into a PMN-like state, adherent, IL-8 primed PMNs are able to internalize Opa- Gc. PMNs possess a variety of receptors found in cytoplasmic granules, which are exposed on the plasma membrane when granules are exocytosed. Comparing surface composition of suspension, HL-60 cells, and adherent, IL-8 primed PMNs may identify potential receptors which account for Opa- Gc internalization in adherent, IL-8 primed PMNs.

Finally, as an alternative to receptor mediated internalization, Opa- Gc could be internalized by macropinocytosis. Macropinocytosis is an actin-dependent process which forms planar or circular cup-shaped membrane ruffles to internalize fluid and small particles in a nonspecific manner. Macropinocytosis can internalize particles less than $1 \mu \mathrm{m}$ in diameter (Lim et al., 2011). Gc ranges in size from $0.5-1 \mu \mathrm{m}$; therefore, membrane 
ruffling may be sufficient to internalize Gc. In preliminary support of this hypothesis, our unpublished observations demonstrate that adherent, IL-8 primed PMNs infected with Gc display large membrane ruffles (Figure 36). In order to test this hypothesis, future experiments could examine Opa- Gc internalization in the presence of macropinocytosis inhibitors such as amiloride (EIPA) and dimethyl amiloride (DMA).

Characterizing and defining the mechanism of Opa- Gc internalization in PMNs is an essential question for future experiments to address. Understanding Opa- Gc internalization may explain the discrepancy in the current literature examining the requirement for Opa proteins in nonopsonic internalization of Gc by PMNs. These experiments will also provide preliminary data for further characterization of the mechanism used by Gc to obtain residence in an immature phagosome which avoids fusion with primary granules.

\subsection{PMN granule components with antigonococcal activity}

Our data indicate that Opa+ Gc reside in mature phagosomes with both PMN primary and secondary granule components, while Opa- Gc fuse only with secondary granules and delay fusion with PMN primary granules. We observed that decreasing primary granule fusion with Opa+ Gc phagosomes enhanced Opa+ Gc survival in PMNs, and conversely, increasing primary granule fusion with Opa- Gc phagosomes resulted in decreased intracellular Gc survival. These data indicate that PMN primary granules contain components with antigonococcal activity. While some of the components contained in PMN granules have been examined for antigonococcal activity in vitro, few have been tested for activity in the context of the PMN phagosome. 
PMN primary and secondary granules contain a variety of antimicrobial

components. Secondary (specific) granules contain gp9 $1^{\text {phox }}$ and $\mathrm{p} 22^{\text {phox }}$ NADPH oxidase subunits, and oxygen-independent antimicrobial products such as lactoferrin and hCAP18, the precursor to the cathelicidin LL-37. Primary granules contain the majority of PMN antimicrobial products, including myeloperoxidase (MPO) to generate hypochlorous acid, $\alpha$-defensin antimicrobial peptides, bactericidal-permeabilityincreasing protein (BPI), and the serine proteases cathepsin G, neutrophil elastase, and proteinase 3 (Borregaard et al., 2007). Of these granule components Gc is resistant to $\alpha$ defensin antimicrobial peptides (Qu et al., 1996) and sensitive to BPI, cathepsin G, and LL-37 in vitro (Casey et al., 1985, Shafer et al., 1986a, Shafer et al., 1998).

Our results point to an important role for PMN proteases in killing Opa+ and Opa- Gc in mature PMN phagosomes. PMN proteases could function to mediate Gc killing directly and/or indirectly. The protease Cathepsin $\mathrm{G}$ has been shown to degrade porin and Opa proteins (Rest et al., 1981, Shafer et al., 1987). Therefore, PMN proteases could act directly by degrading Gc surface components. Additionally, by cleaving products such as hCAP18 to the active form LL-37 which has antigonococcal activity, proteases could also mediate Gc killing indirectly (Faurschou et al., 2003, Shafer et al., 1998). Therefore, future experiments should first explore susceptibility of Gc to known PMN proteases including neutrophil elastase, protease 3, and NSP4 (Faurschou et al., 2003, Borregaard et al., 2007, Perera et al., 2012). The contribution of these components to killing Gc in PMNs could be examined by using inhibitors to individual proteases. Identification of unknown PMN granule components possessing antigonococcal activity could be identified by exposing Gc to fractions of a PMN granule extract which contains 
proteins extracted from PMN granules (Rest, 1979, Rest et al., 1977). Fractions with antigonococcal activity could then be analyzed by mass spectrometry to identify individual components with antigonococcal activity. Identifying PMN components with antigonococcal activity will determine how PMNs kill Gc and also allow for investigation of mechanisms used by Gc to avoid killing by these antimicrobial products.

\subsection{Gc Replication inside PMNs}

While Gc survival in PMNs has been well documented, whether Gc is capable of replicating inside PMNs remains a critical question in the field. PMNs normally have a short lifespan; however, Gc infection with PMNs delays apoptosis (Simons et al., 2006) suggesting replication inside PMNs would be advantageous. Determining if and where Gc replicate inside PMNs would increase our current understanding of Gc pathogenesis and how Gc defend against PMN mediated killing.

Initial experiments investigating replication used modified gentamicin protection assays to demonstrate an increase in Gc CFU over time in PMNs (Veale et al., 1976, Veale et al., 1979, Casey et al., 1980). Experiments using viability dyes and electron microscopy also observed an increased number of viable intracellular Gc at later time points during infection (Simons et al., 2005, Criss et al., 2009). The primary limitation to these assays is the inability to control for external Gc replication. Modified gentamicin protection assays depend on the ability of antimicrobial agents to kill the total external bacterial population without affecting internal bacterial survival. The studies using viability dyes and electron microscopy did not remove the extracellular population of bacteria; therefore, continued phagocytosis of bacteria could account for increased numbers of intracellular, viable bacteria. Due to the experimental limitations of these 
experiments, additional investigation is required to definitively demonstrate intracellular replication.

In order to address this important question, I performed preliminary studies with EdU, a nucleoside analog of thymidine which would be incorporated into bacterial DNA during replication and plating assays examining long term survival of Gc inside PMNs (Appendix C), but results were inconclusive. An alternative to these experiments would be to conduct live imaging experiments using fluorescent Gc to directly view Gc replication or use RT-PCR on total RNA isolated from Gc infected PMNs to compare the amount of bacterial ribosomal RNA over time (Belland, 2003). If there is intracellular Gc replication, there would be an increase in total bacterial ribosomal RNA. My initial experiments investigated the ability of Opa- Gc to survive and replicate in PMNs. However, 10-20\% of internalized Opa+ Gc remain viable inside PMNs after 1 hour. Survival and replication of this population of Opa+ Gc in PMNs which resists killing by antimicrobial products may be sufficient to maintain Gc colonization and persistent infection. Future experiments to investigate Gc replication inside PMNs would significantly expand our understanding of Gc pathogenesis.

Defining the replication compartment would provide important insight into how Gc survives inside PMNs. Immunofluorescence can be used to determine if Gc is replicating in an immature or mature phagolysosome. If Gc are capable of surviving long term or replicating inside mature phagolysosomes, this would suggest that Gc has modulated its gene expression in order to defend against the antimicrobial components found within the phagosome. Since we observed that Opa- Gc phagosomes delay, not 
block, fusion with primary granules, Opa- Gc would eventually reside in mature phagolysosomes. This finding supports the hypothesis that long term survival and replication of Gc in PMNs would require upregulation of genes whose products help resist PMN non-oxidative killing. Future experiments examining Gc gene expression prior to and post exposure to PMNs would increase our knowledge regarding how Gc defend against PMN killing. This may also prompt interesting new questions for investigation including, what are the PMN signals necessary to alter Gc gene expression, and are these genes required for both survival and replication in PMNs?

One other key question to address is whether Gc continue to persist in intact PMNs at later time points, or if viable Gc are released into the extracellular environment upon PMN death. The release of viable Gc into the external environment would allow for colonization of new sites in the infected individual and new individuals. In order to test this hypothesis, PMNs with attached and internalized Gc could be transferred to an epithelial cell monolayer. Then immunofluorescence could be used to monitor epithelial cell infection over time. Experiments addressing the long term survival and replication of Gc in PMNs will expand our overall knowledge of gonorrheal disease progression and transmission.

\subsection{The role of Opa proteins in Gc interactions with PMNs and during gonorrheal infection}

Gc has approximately 11 opa genes encoding 7-8 distinct Opa proteins (Connell et al., 1990, Dempsey et al., 1991). Opa proteins undergo phase variation due to slipped strand mispairing of pentameric nucleotide repeats in the signal sequence-encoding portion of opa genes, at a frequency of $10^{-3}$ to $10^{-4}$ variants/cell/generation in vitro (Stern 
et al., 1986, Murphy et al., 1989). Therefore, both in in vitro cultures and during infection, there is a mixed population of bacteria expressing no Opa proteins, one Opa protein, or multiple Opa proteins. Opa proteins display binding affinity for CEACAMs and/or HSPG. Binding to CEACAMs and HSPGs allows for Gc adherence and internalization into host cells but via different signaling pathways (Wang et al., 1998, Swanson, 1978, Vandeputte-Rutten et al., 2003, de Jonge et al., 2003, Malorny et al., 1998, Sadarangani et al., 2011, van Putten et al., 1995, Freissler et al., 2000, Hauck et al., 2003). Several questions regarding the role of Opa proteins in Gc survival in PMNs and during gonorrheal infection remain. First, how does Opa protein expression direct Gc to a mature phagolysosome? Second, how do different Opa proteins affect PMN activation and Gc survival? Third, Does PMN challenge affect Opa protein phase variation? Finally, what are the advantages to Gc expression of Opa proteins during gonorrheal infection?

\subsubsection{Phagosome Maturation}

Opa+ Gc have reduced survival in PMNs compared to Opa- Gc (Criss et al., 2009, Rest et al., 1982, Virji et al., 1986, Ball et al., 2013). We have demonstrated that the Opa+ Gc survival defect in PMNs is due to residence in a mature phagolysosome containing both primary and secondary granule components. In contrast, our data demonstrate the novel finding that Opa- Gc delay phagosome fusion with primary granules to remain viable inside PMNs. Opa protein engagement of CEACAM receptors stimulates signaling via Syk, PI3K, Rac GTPase, and Src-family kinases. All of these signals have been shown to regulate fusion of PMN granules with target membranes, particularly for exocytosis (Sadarangani et al., 2011, Lacy, 2006). We observed that of these signals, Src-family kinase signaling is important for stimulating phagosome fusion 
with primary granules. Inhibition of Src-family kinase members was sufficient to rescue survival of Gc in PMNs, and stimulation of Src-family kinase members was sufficient to decrease survival of Opa- Gc in PMNs.

We hypothesize that due to differential activation of Src-family kinase members, Opa+ Gc reside in mature phagosomes while Opa- Gc reside in immature phagosomes. In support of this hypothesis, we observed reduced localization of the primary granule associated Src-family kinase member Hck to Opa- Gc phagosomes compared to Opa+ Gc phagosomes. In order to characterize the mechanism of Opa- Gc residence in immature phagosomes, future experiments should investigate activation of Src-family kinase signaling in PMNs in response to infection with Gc. Western blotting for phosphorylated Src-family kinase members could be used to compare activation of Src-family kinase signaling for PMNs infected with Opa+ or Opa- Gc. If differences in activation of Srcfamily kinase members are observed, additional experiments can be conducted to explore if infection of PMNs with Opa- Gc fails to stimulate Src-family kinase member signaling or potentially inhibits Src-family kinases due to activation of phosphatases. Signals downstream of Src-family kinases, such as the activation of p38 MAP kinase, have been implicated in the regulation of PMN granule exocytosis (Mocsai et al., 2000). Future experiments could investigate the role of p38 MAP kinase in regulating phagosome primary granule fusion using inhibitors of p38 MAP kinase and examining activation via western blot analysis. Finally, exploring the route of Opa- Gc internalization via experiments addressed early in the discussion may provide insight into the mechanism used by Gc to avoid phagosome fusion with primary granules and identify potential signaling pathways to examine. Understanding how Gc infection affects activation of 
Src-family kinase signaling will further characterize the mechanisms that lead to Gc residence in mature or immature phagosomes and ultimately impact Gc survival in PMNs.

\subsubsection{Role of different Opa proteins in PMN activation and Gc survival}

Opa engagement of different CEACAMs and HSPGs leads to distinct cellular responses including PMN production of ROS and degranulation (Criss et al., 2009, Sadarangani et al., 2011, Ball et al., 2013, Smirnov et al., 2013, Sarantis et al., 2007, Sarantis et al., 2012). Our observations indicate that expression of different Opa proteins stimulates different amounts of PMN ROS production (Ball et al., 2013). Additionally, binding to CEACAM1 or CEACAM6 individually results in internalization of Gc but does not significantly activate PMNs, while binding to CEACAM3 stimulates uptake and activation (Sarantis et al., 2012). However, it is important to note that engagement of CEACAM1 or CEACAM6 can result in signaling via CEACAM3 to activate PMNs, suggesting these receptors can cooperate in order to stimulate PMN responses (Sarantis et $a l ., 2012)$. These data provide evidence to indicate that Gc expression of different Opa proteins that have specificity for different cellular receptors could affect PMN activation and consequently Gc survival. Our current studies investigated the survival defect of Opa+ Gc in PMNs using a Gc strain expressing one Opa protein, OpaD, that binds CEACAM1 and CEACAM3. Future studies should investigate how Opa protein engagement of different CEACAMs or HSPG affects PMN activation and Gc survival in PMNs.

The Opa- Gc strain generated in our lab containing in frame deletions of all 11 opa genes provides experimental advantages for addressing the role of Opa proteins in 
Gc survival in PMNs. First, it allows for examination of Gc interactions with PMNs in the absence of Opa protein expression. Second, it provides a strain background in which individual Opa proteins or different combinations of Opa proteins can be locked on for expression. The contribution of each individual Opa protein to Gc interactions with PMNs can be examined using this system. Gc strains expressing different combinations of Opa proteins can be used to dissect more complex interactions with PMNs. We observed that coinfection of PMNs with Opa- and Opa+ Gc resulted in reduced survival of Opa- Gc in PMNs. This suggests that Opa+ Gc interactions with PMNs stimulate signaling that alters Opa- Gc survival in PMNs. Future experiments can alter the ratio of Opa- and Opa+ Gc, extend the time course of infection, and examine coinfection of PMNs with Gc strains expressing different Opa proteins. The ability to have defined Gc strains expressing one or any combination of Opa proteins provides a valuable tool to dissect PMN activation due to CEACAM and/or HSPG engagement and determine how the balance of these signals affects Gc survival in PMNs.

Using the Opa- Gc strain, we can also determine if Opa protein phase variation occurs during PMN challenge. In the Opa- Gc background, we can engineer a strain with an opa gene retaining the pentameric repeats that undergo phase variation. This strain will be referred to as Opa+ PV Gc. PMNs can then be infected with Opa+ PV Gc which are phase-off and lack Opa protein expression, or with Opa+ PV Gc which are phase-on and therefore express Opa proteins. The number of Opa- and Opa+ bacteria in the total recovered $\mathrm{CFU}$ over time can be evaluated using light microscopy to distinguish translucent (Opa-) and opaque (Opa+) colonies. If we observe that Opa protein expression is either phase varied off or on over the course of infection, this would suggest 
that Gc can vary Opa protein expression in PMNs. Experiments investigating Opa protein phase variation during PMN challenge will increase our understanding of how Gc dynamically phase varies Opa protein expression to survive in the presence of PMNs.

\subsubsection{Advantages and Disadvantages of Opa protein Expression during gonorrheal infection \\ Opa + Gc are recovered from naturally and experimentally infected individuals} (James et al., 1978, Hobbs et al., 2011). Inoculation of male volunteers with Opa- Gc results in the recovery of predominantly Opa+ Gc. Gc expressing certain Opa proteins are recovered more (FA1090 B, F, E/K, and I) or less frequently (FA1090 A, D, and C) from infected individuals. The recovery of Opa+ Gc isolates in vivo suggests there is selection for Opa protein expression; however, an alternative is that Opa+ Gc are just more readily shed. A primary question which remains in the field is why are Opa+ Gc selected for in vivo despite being readily killed by PMNs (Figure 37)?

At least two roles for Opa proteins have been described during infection: firm attachment to epithelial cells (Merz et al., 2000) and resistance to proteases such as metalloproteases and cathepsin expression or hormones found in cervical secretions (Jerse, 1999, Simms et al., 2006, Salit, 1982). Our data indicate that Opa+ and OpaFA1090 Gc have similar susceptibility to cathepsin G, suggesting a role for metalloproteases. We hypothesize that in addition to these known advantages, expression of Opa proteins may confer a survival advantage for Gc when encountering PMNs.

First, Opa+ Gc may promote increased recruitment and activation of PMNs. This may result in increased tissue damage, which would allow for bacteria to readily cross the epithelial barrier. In support of this possibility, some Opa+ Gc induce a potent PMN 
oxidative burst (Criss et al., 2008, King et al., 1978b, Kupsch et al., 1993, Fischer et al., 1988, Virji et al., 1986, Rest et al., 1982) and also stimulate greater granule exocytosis compared to Opa- Gc (Sarantis et al., 2007). The increased ROS and PMN antimicrobial components may induce tissue damage to increase bacterial dissemination. Second, stimulation of the oxidative burst may serve as a signal for $\mathrm{Gc}$ to upregulate defensive genes. Gc pre-exposed to hydrogen peroxide have increased survival in PMNs compared to unexposed Gc (Criss et al., 2009) suggesting that Gc upregulate genes to defend against PMN killing in response to ROS. RecN and Ngo1686 are two genes upregulated in response to hydrogen peroxide exposure (Stohl et al., 2005). RecN and Ngo1686 defend against both oxidative and nonoxidative damage in vitro (Stohl et al., 2012, Stohl et al., 2006, Davidsen et al., 2005, LeCuyer et al., 2010, Stohl et al., 2005, Stohl et al., 2013). Expression of RecN and Ngo1686 increase Gc survival after PMN challenge (Stohl et al., 2013, Stohl et al., 2005, Criss et al., 2009). Together these data support the hypothesis that the oxidative burst is a signal for the upregulation of genes, including RecN and Ngo1686, that contribute to Gc survival in PMNs. Third, PMN recruitment provides a survival and/or replication niche for Gc. Our data indicate a population of Gc are capable of surviving intracellularly in PMNs. Therefore, PMNs may transport viable Gc across the epithelial barrier or to new individuals to aid in dissemination and transmission (Criss et al., 2012).

The primary disadvantage observed for Opa protein expression is Opa+ Gc have reduced survival in PMNs. Opa+ Gc are internalized by PMNs and are readily killed due to residence in a degradative phagolysosomes containing oxidative and non-oxidative components (Chapter 3). Despite having an initial survival defect in PMNs, if Opa+ Gc 
are observed to replicate within PMNs, this may compensate for this initial disadvantage (4.4). Therefore, during gonorrheal infection Opa+ Gc may be initially killed but have an overall survival advantage due to the ability of Opa+ Gc to replicate inside PMNs.

In contrast, Opa- Gc fail to induce an oxidative burst and reside in an immature phagosome to survive intracellularly in PMNs (Johnson et al., 2013b, Criss et al., 2008, Criss et al., 2009, Ball et al., 2013). During infection, Opa- Gc are recovered from females during menses and from the fallopian tubes of women with disseminated infection (Jerse, 1999, Jerse et al., 1994). The ability of Opa- Gc to avoid stimulation of potent inflammation and tissue damage may serve an important function in survival of Gc in PMNs and during certain points of gonorrheal infection. Therefore, a combination of Opa+ and Opa- Gc may be required during infection in order to maintain colonization and resist clearing by the host immune system.

\subsection{Overall Conclusions}

$\mathrm{Gc}$ is a remarkable pathogen in that infection stimulates the recruitment of abundant PMNs to the site of infection; however, Gc are able to defend against PMN mediated killing and remain viable (Wiesner et al., 1980). Viable Gc have been observed inside PMNs infected in vitro (Simons et al., 2005, Criss et al., 2009), but the mechanism of Gc intracellular survival has remained unknown. The results discussed here present the novel finding that Opa- Gc reside in an immature phagosome in order to remain viable inside PMNs. Residence in an immature phagosome is not dependent on active Gc

processes. Instead altering the surface composition of Gc by expression of Opa proteins is sufficient to drive Gc into a mature phagolysosomes. Previous research has documented the survival defect of Opa+ Gc in PMNs. This work provides data to demonstrate that the 
survival defect of Opa+ Gc is due to stimulation of phagosome fusion with primary granules via Src-family kinase signaling. Together these findings support a model in which the route of Gc internalization affects phagosome maturity and Gc survival in PMNs. The knowledge acquired in these studies expands our current understanding of Gc interactions with PMNs during infection and most importantly assists in directing future experimental pursuits. Addressing the key questions discussed above will greatly enhance our current understanding of gonorrheal infection and may potentially reveal novel therapeutic targets. 


\subsection{Figures for Chapter 4}

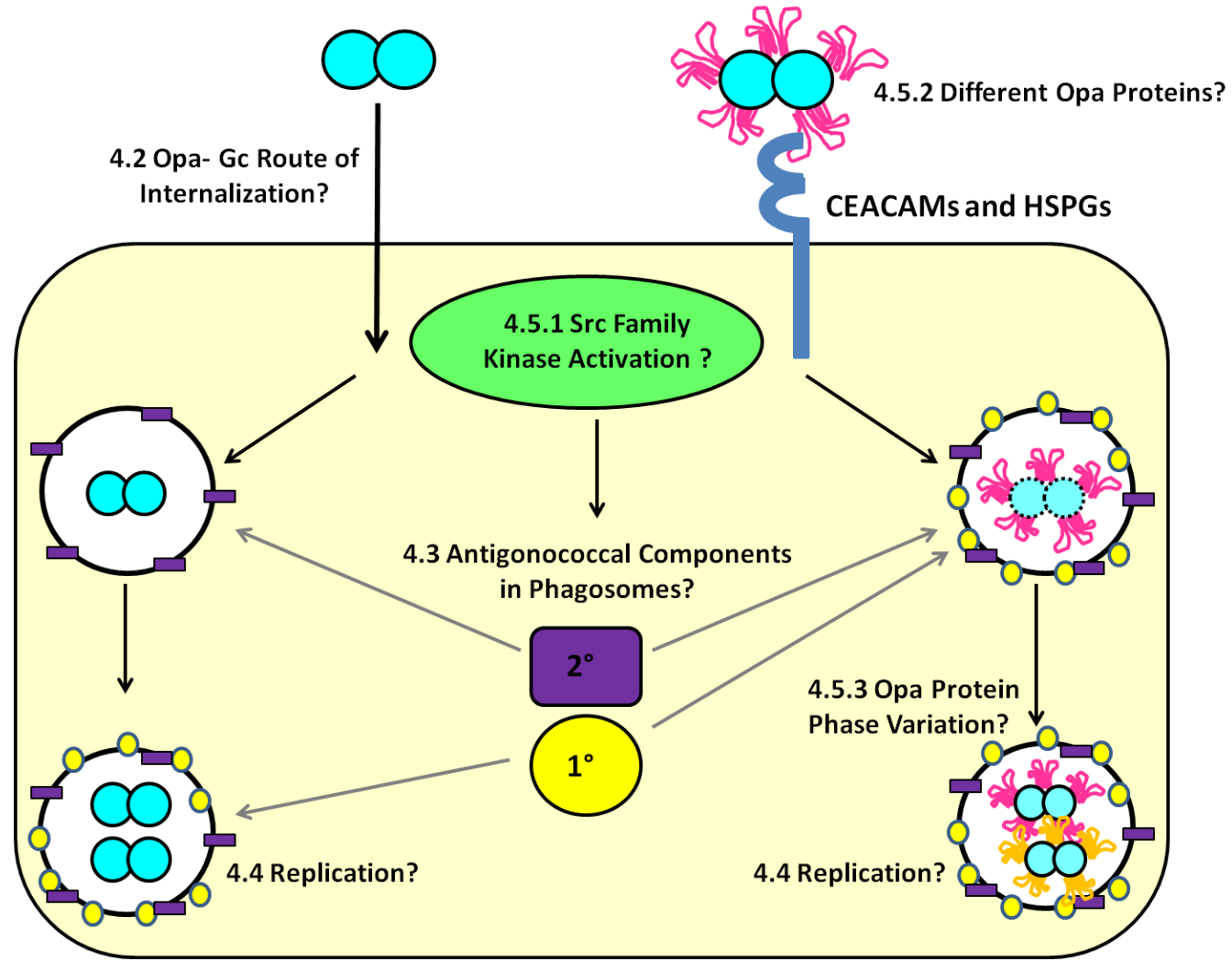

Figure 35: Essential questions for future studies to address 
(4.2) Our results indicate that Opa- Gc survive intracellularly in an immature phagosome inside PMNs. How Opa- Gc are internalized is an important remaining question. Addressing this question would assist in defining the mechanism of delayed fusion of primary granules with Opa- Gc phagosomes. (4.3) We identified serine proteases as an important PMN granule component with antigonococcal activity. PMN granules contain several proteases including neutrophil elastase, proteinase 3, cathepsin G, and NSP4. The future experiments should investigate the contribution of each of these proteases to Gc killing as well as identify novel granule components with antigonococcal activity. (4.4) A percent of intracellular Opa- and Opa+ Gc remain viable inside PMNs. Section 4.4 discusses the importance of investigating if Opa- and Opa+ Gc are capable of replicating inside PMNs and the advantages intracellular replication would serve during gonorrheal infection. (4.5.1) Our results indicate Src-family kinase signaling is important for phagosome fusion with primary granules. Future studies should examine if Src-family kinase signaling is differentially activated due to Opa protein expression. (4.5.2) These studies examined the role of Opa protein expression in Gc survival in PMNs using a Gc strain expressing one Opa protein. Section 4.5.2 discusses the importance of investigating how different Opa proteins affect PMN activation and Gc survival in PMNs. (4.5.3) Gc is capable of individually phase varying each of the 11 opa genes. The factors during gonorrheal infection which affect selection for expression of different Opa proteins and potentially increase Opa protein phase variation are not well understood. Future experiments should examine if PMN challenge influences selection of Gc expressing different Opa proteins or stimulates increased phase variation of Opa proteins. 


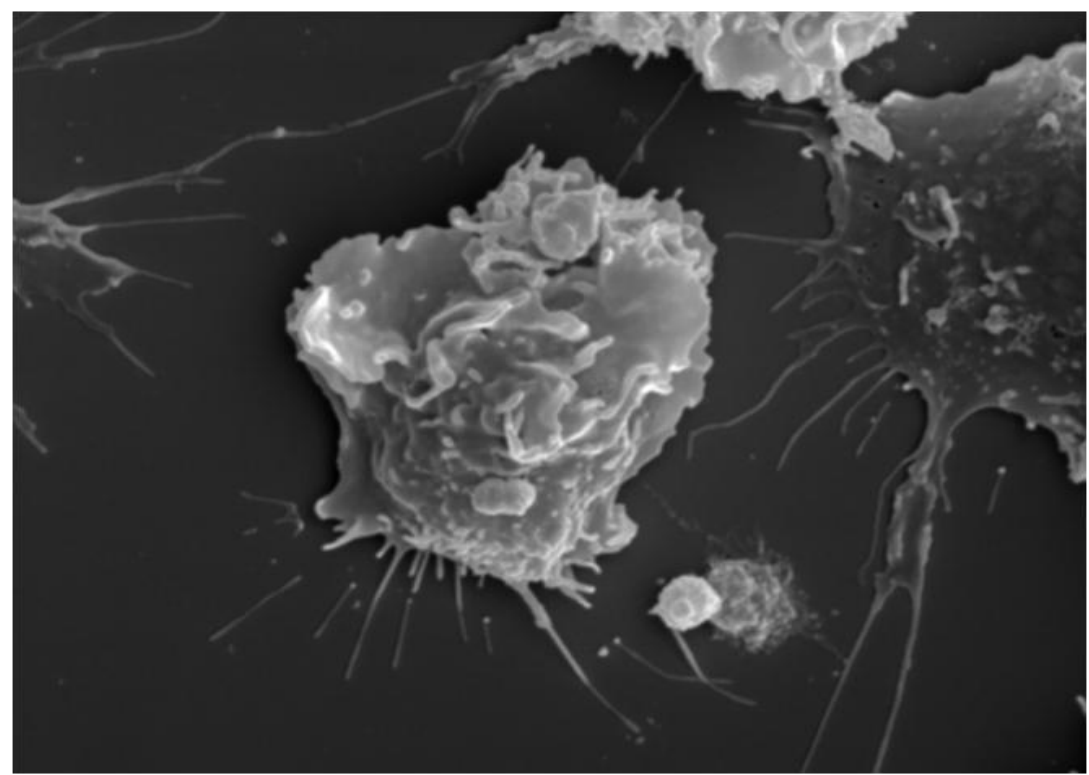

Figure 36: SEM image of Gc infected PMNs

Scanning electron micrograph of adherent, IL-8 primed PMNs infected with Gc. Image shows large membrane ruffles and protusions in response to Gc infection. 


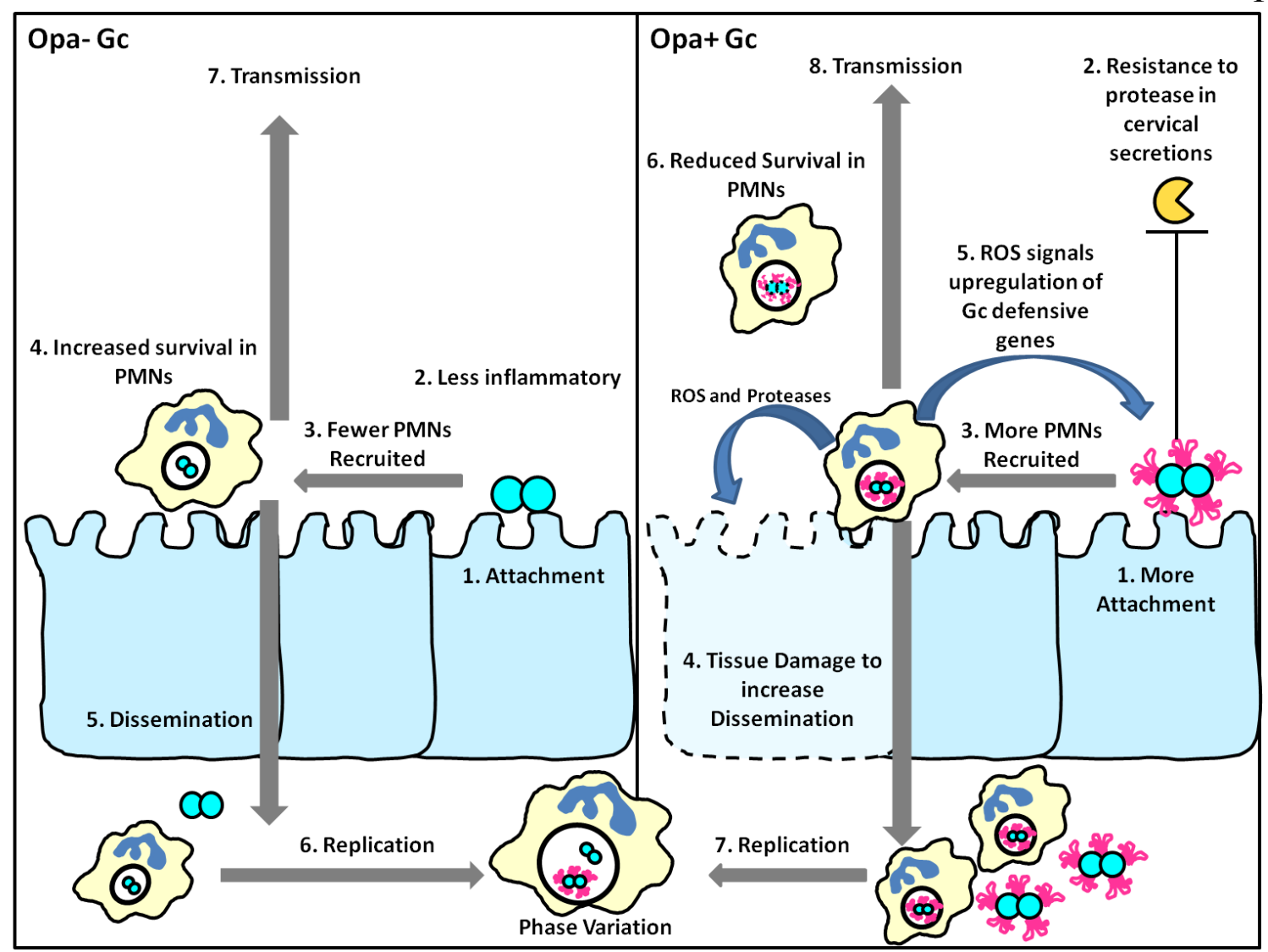

Figure 37: Advantages for Opa- and Opa+ Gc during gonorrheal infection 
During gonorrheal infection there would be a mixed population of Gc expressing no Opa proteins, one Opa protein, or multiple Opa proteins. Opa- and Opa+ Gc could each serve advantages during gonorrheal infection. Opa- Gc and Opa+ Gc are both able to attach and colonize epithelial cells; however, Opa protein expression increases adherence to and invasion of epithelial cells. Opa protein expression also provides resistance to proteases found in cervical secretions. Gc colonization stimulates release of cytokines to induce $\mathrm{PMN}$ recruitment. Opa+ Gc stimulate increased PMN recruitment compared to Opa- Gc. Opa+ Gc stimulation of PMN granule exocytosis and production of ROS would result in increased tissue damage which could increase dissemination. Additionally, production of ROS could serve as a signal for Gc to upregulate genes which provide resistance to PMN antimicrobial products. While Opa+ Gc have an initial survival defect in PMNs compared to Opa- Gc, a population of both Opa+ and Opa- Gc are capable of surviving inside PMNs. Survival of Gc in PMNs would provide two main advantages. First, PMNs may serve as survival and replication niche which is protected from other aspects of the host immune response. Second, PMNs could carry viable Gc across the epithelial barrier or to new individuals in order to increase dissemination and transmission. The balance of Opaand Opa+ Gc may be important during gonorrheal infection due to the advantages provided by each bacterial phenotype. Therefore, during the course of infection Gc may phase Opa protein expression in order to maintain persistent infection and avoid killing by the host immune response. 


\section{Appendix A: Fluorescence microscopy methods for determining the viability of bacteria in association with mammalian cells}

This chapter is a modified version of the previously published article, "Fluorescence microscopy methods for determining the viability of bacteria in associate with mammalian cells" [J Vis Exp., 79, 2013. doi: 10.3791/50729].

\subsection{Introduction}

There is a dynamic interaction and co-evolution between bacteria and the hosts in which they reside. Bacteria have evolved adherence organelles, secretion systems, and/or the ability to produce toxins that enable their productive infection of host phagocytic and non-phagocytic cells. The bacteria must also contend with recognition and antimicrobial activities of the host immune system. The host immune system is comprised of innate and adaptive components including physical and chemical barriers, immune cells, the complement system, and other components of humoral immunity. While many bacteria are susceptible to killing and clearance by the multilayered host immune response, some pathogenic and opportunistic bacteria have evolved mechanisms to infect a variety of host cells and subvert clearance by the host immune response (Woolard et al., 2008). Neisseria gonorrhoeae is one example of a bacterial pathogen which is highly adapted to persist in its human host. N. gonorrhoeae readily colonizes the luminal surfaces of mucosal epithelial cells of the urogenital tract, pharynx, conjunctiva, and rectum. Colonization triggers the abundant recruitment of PMNs at mucosal sites. PMNs are professional phagocytes which possess a variety of antimicrobial processes to kill microorganisms; however, $N$. gonorrhoeae is capable of surviving in the presence of PMNs (Johnson et al., 2011, Simons et al., 2005, Seib et al., 2005, Criss et al., 2009). 
Understanding how bacterial pathogens such as $N$. gonorrhoeae subvert, suppress, and hijack the immune response to ultimately survive in normally hostile host environments is crucial to the development of new therapies for combating infectious diseases.

Experimental protocols often used to investigate bacterial survival in host cells include colony count assays, gentamicin protection assays, and electron microscopy. In colony count assays, a population of infected cells is lysed (for instance, with a detergent to which the bacteria are resistant) to liberate the bacteria. The lysates are diluted and plated on agar-based media, and colony-forming units in the lysates are enumerated for each time point and/or experimental condition. This approach reports the viability of the entire bacterial population but is not capable of differentiating between intracellular and extracellular survival. A variation on the colony count assay, the gentamicin protection assay, specifically measures intracellular bacterial survival, based on the inability of the antibiotic gentamicin to cross the eukaryotic plasma membrane (Edwards et al., 2011). However, this assay is dependent on the bacteria being susceptible to killing by gentamicin (or another antibiotic that is similarly eukaryotic membrane-impermeant) and the inability of the antibiotic to have access to internal bacteria. Therefore, a gentamicin protection assay may not be effective for examination of all bacterial species or when examining bacterial survival in highly pinocytic cells such as PMNs. Neither of these approaches reveals the subcellular localization or other behavior of individual bacteria (e.g. if the bacteria form aggregates or microcolonies that behave differently from individual bacterial cells). Another frequently used approach to examine the viability of individual external and internal bacteria is thin-section transmission electron microscopy (TEM). This approach is advantageous in that it can provide information regarding the 
location of the bacteria in host cells (e.g., phagosome, cytoplasm, autophagosome), which can be further investigated by immunoelectron microscopy with gold-coupled antibodies against subcellular markers. However, electron microscopy is not especially sensitive at assessing bacterial viability. When embedded sections are stained with uranyl acetate, lead citrate, or other electron-dense reagents and imaged by electron microscopy, electron-dense bacteria are considered viable and electron-lucent nonviable (Bozzola, 2007, Dorward, 2008). However, this assumption overestimates bacterial viability, since only those dead bacteria with severely disrupted membranes and devoid of cytoplasm appear electron-lucent. In addition, some bacterial species may display a range of electron densities depending on their stage of growth, making it difficult to determine viability.

As an alternative or in addition to these widely used methods, here we provide protocols and rationale for the use of fluorescent dyes that indicate bacterial viability to assess the survival of bacteria attached to and internalized by host cells. To identify extracellular bacteria, infected cells are first exposed to a fluorescent reagent, such as a lectin or bacteria-specific antibody. The infected cells are then permeabilized and exposed to DNA-specific dyes that are differentially accessible to bacteria with intact vs. degraded membranes, as a surrogate for bacterial viability. In the first protocol, the membrane permeable dye SYTO9 identifies the total bacterial population, while propidium iodide is only accessible to those bacteria which have compromised membranes and are thus considered nonviable. Propidium iodide and SYTO9 have been used to evaluate bacterial viability in biofilms, discriminate pathogenic from nonpathogenic bacteria, and enumerate viable water-borne bacteria (Yang et al., 2010, 
Mueller et al., 2007, Shen et al., 2012, Hoefel et al., 2003). In the second protocol, 4', 6'-diamidino-2-phenylindole (DAPI) identifies total bacteria, while SYTOX Green is only accessible to the nonviable population. These viability dye pairs can be combined with immunofluorescence to determine each bacterium's location in relation to a protein of interest, for instance to define bacterial subcellular localization. The use of these assays provides key insight into the interactions which result in bacterial killing or survival during infection of host cells. The protocols outlined in this article were used to assess the viability of $N$. gonorrhoeae that is attached to and inside primary human PMNs, including in different populations of PMN phagosomes (Oh et al., 2008, Allen, 2007, Criss et al., 2009). However, these protocols can be applied to assess viability of gram-positive and gram-negative bacteria in professional phagocytes, non-professional phagocytes, and protozoa (Kaplan et al., 2006, Chow et al., 2010, Thulin et al., 2006, Kubica et al., 2008, Swords et al., 2006, Tamilselvam et al., 2006, Martinez et al., 2009, Botha et al., 2012, Allen et al., 2000, Smith et al., 2012).

\subsection{Materials and Methods}

\subsubsection{Assessing bacterial viability with propidium iodide and SYTO9.}

5.2.1.1 Infect cells that are adherent to $12 \mathrm{~mm}$ diameter circular glass coverslips in 24 well plates with bacteria of interest. Do NOT fix the cells with aldehydes or organic solvents.

5.2.1.2 Rinse cells once gently in 0.1M 3-(N-morpholino) propanesulfonic acid (MOPS), pH 7.2 containing $1 \mathrm{mM} \mathrm{MgCl} 2$ (MOPS/ $\mathrm{MgCl}_{2}$ ). 
5.2.1.3 Incubate cells for 10 minutes in the dark at room temperature with Alexa Fluor 647-coupled antibody or lectin that binds to the bacterial species of interest, in MOPS/ $\mathrm{MgCl}_{2}$, to detect external bacteria.

NOTE: Conduct controls with live and dead bacteria, in the absence of host cells, to show that the lectin or antibody of interest binds all bacteria regardless of viability (Figure 38).

5.2.1.4 Rinse cells two times with $\mathrm{MOPS} / \mathrm{MgCl}_{2}$.

5.2.1.5 Aspirate media from cells and add 0.5 ml Live/Dead Staining Solution. Live/Dead Staining Solution is $5 \mu \mathrm{M}$ SYTO9, $30 \mu \mathrm{M}$ propidium iodide, and $0.1 \%$ saponin final concentrations in $\mathrm{MOPS} / \mathrm{MgCl}_{2}$.

5.2.1.6 Incubate cells for 15 minutes at room temperature in the dark.

5.2.1.7 Rinse cells two times in MOPS/ $\mathrm{MgCl}_{2}$.

5.2.1.8 Invert coverslips facedownonto glass slides and seal with clear nail polish. Do not use mounting media.

5.2.1.9 Acquire images within 30 minutes, using a fluorescence microscope with filter sets compatible with green, red, and far-red image acquisition.

NOTE: Both conventional fluorescence microscopy and confocal laser scanning microscopy can be used. After 30 minutes the fluorescent dyes begin to leak from the bacteria and the data acquired is no longer accurate. Images shown in this article were acquired on a Nikon Eclipse E800 upright fluorescence microscope with Hamamatsu Orca-ER digital camera using Openlab software. Fluorescence of Alexa Fluor 647 was detected using a filter with excitation wavelength of 590-650 $\mathrm{nm}$ and an emission filter of $663-735 \mathrm{~nm}$, and is false- 
colored blue. Fluorescence from propidium iodide was detected using a filter with excitation wavelength of 540-580 nm and an emission filter of 600-660 nm. Fluorescence from SYTO9 was detected using a filter with excitation wavelength of 465-495 $\mathrm{nm}$ and an emission filter of 515-555 nm.

5.2.1.10 This protocol will result in images where external nonviable bacteria appear blue + red, internal nonviable bacteria appear red only, external viable bacteria appear blue + green, and internal viable bacteria appear green only. Count by eye the number of bacteria which are external nonviable, internal nonviable, external viable, and internal viable.

5.2.1.11 Calculate the percent of external viable bacteria by dividing the number of external viable bacteria by the total number of external bacteria (viable plus nonviable). Calculate the percent of internal viable bacteria by dividing the number of internal viable bacteria by the total number of internal bacteria (viable plus nonviable) (Figure 41).

5.2.1.12 There are two essential controls to perform with this protocol. First, validate that all nonviable bacteria are propidium iodide-positive and all live bacteria are SYTO9-positive. A mid-logarithmic culture of bacteria (in the absence of any host cells) should be $>95 \%$ SYTO9-positive. Shown in Figure 39 is a midlogarithmic culture of $N$. gonorrhoeae at $10^{8}$ colony forming units per ml which was incubated with Live/Dead Staining Solution. To generate a population of dead bacteria collect $2 \times 10^{8}$ bacterial colony forming units in a $1.5 \mathrm{ml}$ microfuge tube and add $70 \%$ isopropanol for 10 minutes. Pellet the bacteria in a microfuge and wash the bacteria twice in PBS to remove residual isopropanol. 
Then follow protocol steps 1.5-1.7.Add $5 \mu \mathrm{l}$ of bacterial suspension to a glass slide and overlay with a coverslip. Image samples as in protocol step 1.9. Under these conditions, $100 \%$ of the population should be propidium iodide-positive (Figure 39). In some bacterial species, propidium iodide may not completely overwhelm SYTO9 staining, and nonviable bacteria may appear yellow or orange. Second, validate that both propidium iodide and SYTO9 can enter permeabilized, infected host cells. For phagocytic cells like PMNs, expose the cells to isopropanol-killed bacteria and ensure that $100 \%$ of the intracellular bacteria are propidium iodide-positive (Figure 40). For nonphagocytic cells that may not internalize dead bacteria, it may be sufficient to treat infected cells with sodium azide or other cell-permeant antimicrobial agents prior to adding Live/Dead Staining Solution.

\subsubsection{Assessing bacterial viability with SYTOX Green and DAPI.}

5.2.2.1 Label bacteria of interest with $10 \mu \mathrm{g} / \mathrm{ml}$ DAPI in Morse's Defined Medium (Morse et al., 1980) for 20 minutes at room temperature in the dark.

5.2.2.2 Infect cells that are adherent to $12 \mathrm{~mm}$ diameter circular glass coverslips in 24 well plates with DAPI-labeled bacteria. Do NOT fix the cells with aldehydes or organic solvents.

5.2.2.3 Rinse cells once with MOPS/ $\mathrm{MgCl}_{2}$.

5.2.2.4 Incubate cells for 10 minutes at room temperature in the dark with Alexa Fluor 647-coupled antibody or lectin that binds to the bacterial species of interest, in MOPS/ $\mathrm{MgCl}_{2}$, to detect external bacteria. See note in protocol step 1.3 for suggested controls. 
5.2.2.5 Aspirate media from cells and add $0.5 \mathrm{ml} 0.4 \mu \mathrm{M}$ SYTOX Green in $\mathrm{MOPS} / \mathrm{MgCl}_{2}$.

5.2.2.6 Incubate cells for 5 minutes at room temperature in the dark.

5.2.2.7 Rinse cells two times in MOPS/ $\mathrm{MgCl}_{2}$.

5.2.2.8 Wash cells one time in $\mathrm{MOPS} / \mathrm{MgCl}_{2}$ for 5 minutes.

5.2.2.9 Acquire images within 30 minutes with a fluorescence microscope. See protocol step 1.9 for description of microscope, digital camera, and acquisition software.

NOTE: Fluorescence from Alexa Fluor 647 was detected using a filter with excitation wavelength of 590-650 $\mathrm{nm}$ and an emission filter of $663-735 \mathrm{~nm}$, and is falsecolored red. Fluorescence from SYTOX Green was detected using a filter with excitation wavelength of 465-495 $\mathrm{nm}$ and an emission filter of 515-555 nm. Fluorescence from DAPI was detected using a filter with excitation wavelength of $355-375 \mathrm{~nm}$ and barrier filter of $400 \mathrm{~nm}$.

5.2.2.10 This protocol will result in images where external nonviable bacteria appear red + green, internal nonviable bacteria appear green + blue, external viable bacteria appear red + blue, and internal viable bacteria appear blue only (Figure 41). Quantify the percent of external and internal bacteria as described in protocol steps 1.9-1.10.

5.2.2.11 The controls described in protocol step 1.11 should be performed with the DAPI/SYTOX Green dye combination (Figure 39 and Figure 40).

\subsubsection{Assessing bacterial viability alongside subcellular localization.}

5.2.3.1 Label bacteria of interest with $10 \mu \mathrm{g} / \mathrm{ml}$ DAPI in Morse's Defined Medium for 20 minutes at room temperature in the dark. 
5.2.3.2 Infect cells that are adherent to $12 \mathrm{~mm}$ diameter circular glass coverslips in 24 well plates with DAPI-labeled bacteria. Do NOT fix the cells with aldehydes or organic solvents.

5.2.3.3 Rinse cells once with $\mathrm{MOPS} / \mathrm{MgCl}_{2}$.

5.2.3.4 Incubate 10 minutes at room temperature in the dark with Alexa Fluor 647coupled antibody or lectin that binds to the bacterial species of interest, in MOPS/ $\mathrm{MgCl}_{2}$, to detect external bacteria,. See note in protocol step 1.3 for suggested controls.

5.2.3.5 Aspirate media from cells and rinse cells two times in $\mathrm{MOPS} / \mathrm{MgCl}_{2}$.

5.2.3.6 Incubate cells with Alexa Fluor 555-coupled antibody against subcellular marker of interest for 20 minutes, in $\mathrm{MOPS} / \mathrm{MgCl}_{2}$ containing $0.2 \%$ saponin.

5.2.3.7 Rinse cells two times in MOPS/ $\mathrm{MgCl}_{2}$.

5.2.3.8 Wash cells one time in $\mathrm{MOPS} / \mathrm{MgCl}_{2}$ for 5 minutes.

5.2.3.9 Aspirate media from cells and add 0.4 $\mu \mathrm{M}$ SYTOX Green in $\mathrm{MOPS} / \mathrm{MgCl}_{2}$.

5.2.3.10 Incubate cells 5 minutes at room temperature in the dark.

5.2.3.11 Rinse cells two times in $\mathrm{MOPS} / \mathrm{MgCl}_{2}$.

5.2.3.12 Wash cells one time in MOPS/ $\mathrm{MgCl}_{2}$ for 5 minutes.

5.2.3.13 Acquire images of slides within 30 minutes on fluorescence microscope. See protocol step 1.9 for description of microscope, digital camera, and acquisition software.

NOTE: Fluorescence from Alexa Fluor 647 was detected using a filter with excitation wavelength of 590-650 $\mathrm{nm}$ and emission filter of $663-735 \mathrm{~nm}$, and is falsecolored purple. Fluorescence from Alexa Fluor 555 was detected using a filter 
with excitation wavelength of 540-580 nm and an emission filter of 600-660

nm. Fluorescence from SYTOX Green was detected using a filter with excitation wavelength of 465-495 nm and an emission filter of 515-555 nm.

Fluorescence from DAPI was detected using a filter with excitation wavelength of $355-375 \mathrm{~nm}$ and barrier filter of $400 \mathrm{~nm}$.

5.2.3.14 This protocol will result in images where external nonviable bacteria appear purple + green, internal nonviable bacteria appear green + blue, external viable bacteria appear purple + blue, internal viable bacteria appear blue only, and subcellular protein appears red (Figure 41). Quantify the perecent of external and internal viable bacteria as described in protocol steps 1.9-1.10.

5.2.3.15 The controls described in protocol step 1.11 should be performed with the DAPI/SYTOX Green dye combination (Figure 39 and Figure 40).

5.2.3.16 In addition to counting viable and nonviable bacteria, classify each bacteria as either positive or negative for colocalization with the subcellular marker of interest.

5.2.3.17 Calculate the percent of viable bacteria colocalized with the subcellular marker by dividing the number of colocalized viable bacteria by the total number of viable bacteria. Calculate the percent of nonviable bacteria colocalized with the subcellular marker by dividing the number of nonviable colocalized bacteria by the total number of nonviable bacteria.

\subsection{Representative Results}

The protocols outlined were used to examine survival of $N$. gonorrhoeae after exposure to primary human PMNs (Criss et al., 2009, Johnson et al., 2013b). PMNs were 
infected with $N$. gonorrhoeae and processed with protocol 1, using the green-fluorescent viability dye SYTO9 and the red-fluorescent propidium iodide (Figure 41). The dyes were added in the presence of saponin, which sequesters cholesterol to preferentially permeabilize host cell plasma membranes, not $N$. gonorrhoeae membranes. Other detergents may need to be tested if the membranes of the bacteria of interest contain high amounts of cholesterol. All N. gonorrhoeae stain with SYTO9, but only bacteria with compromised membranes stain with propidium iodide. The propidium iodide overcomes the SYTO9 fluorescence, so live bacteria appear green and dead bacteria appear red (Stocks, 2004). In some bacterial species, propidium iodide may not completely overcome SYTO9 staining, resulting in dead bacteria appearing yellow or orange. The ratio of propidium iodide to SYTO 9 may need to be optimized for different bacterial species. Prior to permeabilizing the cells and adding SYTO9 and propidium iodide, an Alexa Fluor 647-coupled soybean lectin was added to the infected cells to detect extracellular N. gonorrhoeae, and the far-red fluorescence signal was false-colored blue. Thus, in this protocol external viable bacteria appear turquoise (blue + green) and external nonviable appear magenta (blue + red). Internal viable bacteria appear green only (arrow), and internal nonviable bacteria appear red only (arrowhead). It is important to note that in infected cells, the eukaryotic cell nucleus will be stained by SYTO9 and propidium iodide (“N," Figure 41A). The external viable, external nonviable, internal viable, and internal nonviable bacteria were quantified from fluorescence images to yield the percent of internalized bacteria and the percent viable bacteria inside and outside host cells. 
Figure 41B is a representative image of $N$. gonorrhoeae-infected PMNs processed with protocol 2, using the viability dyes SYTOX Green and DAPI. This protocol avoids the use of propidium iodide, which fluoresces in both red and ultraviolet channels on the fluorescence microscope. All N. gonorrhoeae stain with DAPI (blue), but only bacteria with compromised membranes stain with SYTOX Green. As described above, before permeabilizing the cells, an Alexa Fluor 647-coupled soybean lectin was added to the infected cells to detect extracellular $N$. gonorrhoeae, whose fluorescence in this case is false-colored red. Therefore, external viable bacteria appear magenta (red + blue) and external nonviable bacteria appear yellow (red + green/blue). Internal viable bacteria appear blue only (arrows), and internal nonviable bacteria appear green/blue (arrowheads). Depending on the relative intensity of DAPI vs. SYTOX Green fluorescence, nonviable bacteria may appear turquoise. Similar to cells processed with propidium iodide and SYTO9, the PMN nucleus is stained by SYTOX Green and DAPI in Figure 41B (indicated by N). Exposure of infected cells to SYTOX Green and DAPI reveals the percent internalization and viability of bacteria in host cells, quantified as described above for propidium iodide and SYTO9. Results from N. gonorrhoeae-infected PMNs processed using SYTOX Green and DAPI are comparable to those obtained with propidium iodide and SYTO9 (Johnson et al., 2013b). As depicted in Figure 41C, protocol 3 is an adaptation of protocol 2, in which the bacterial viability dyes were combined with immunofluorescence for the primary or azurophilic PMN granule protein CD63. This experiment was conducted in order to define the composition of phagosome compartments inside PMNs that contain viable or nonviable $N$. gonorrhoeae (Johnson et $a l .$, 2013b). This protocol requires a microscope for four-color fluorescence capability to 
detect viable bacteria, nonviable bacteria, extracellular bacteria, and subcellular marker. Since the viability dyes are not compatible with aldehyde fixation (our unpublished observations), we performed immunofluorescence without fixation, using an antibody directly coupled to a fluorophore to minimize the time needed to detect the antigen inside cells. This antibody is coupled to the red fluorophore Alexa Fluor 555. As in protocol 2, viable and nonviable bacteria were distinguished using DAPI and SYTOX Green, respectively, and external bacteria were stained using Alexa 647-coupled soybean lectin. External bacteria fluoresce far-red and are false-colored purple. External viable bacteria appear purple + blue and external nonviable bacteria appear purple + green/blue. Internal viable bacteria appear blue (arrow) and internal nonviable bacteria appear green (arrowhead). CD63-positive granules and phagosomes appear red. The PMN nucleus is stained with the DNA specific dyes ("N"). When analyzing these images, each individual bacterium was classified as external vs. internal, viable vs. nonviable, and positive or negative for a ring of CD63 staining. $N$. gonorrhoeae was considered to reside in a PMN CD63-positive phagosome if there is $\geq 50 \%$ of a ring of CD63 fluorescence surrounding the bacteria, and in a CD63-negative phagosome if there is $<50 \%$ of a ring of CD63 fluorescence surrounding the bacteria. The nonviable intracellular bacterium indicated by the arrowhead in

Figure $41 \mathrm{C}$ has a ring of staining for CD63 surrounding the bacteria. This ring indicates primary granules are enriched at this phagosome. The viable intracellular bacterium, indicated by the arrow, lacks staining for CD63, indicating primary granules are not enriched at its phagosome. This technique was used to show a correlation between the viability of $N$. gonorrhoeae and residence in a primary granule-negative phagosome 
(Johnson et al., 2013b). Because this assay tracks both viability and bacterial localization using a protein of interest, it is possible to determine if nonviable and viable bacteria reside in the same or different locations. This information provides important initial insight into mechanisms that contribute to bacterial survival in host cells.

\subsection{Discussion}

Presented here are two protocols that use DNA binding and viability dyes in conjunction with a fluorescent lectin to identify live and dead bacteria attached to and inside human cells. Since both protocols effectively discriminate live from dead bacteria,the choice of which protocol to use depends upon the goal of the experiment. The first protocol uses propidium iodide to detect nonviable bacteria and SYTO9 to detect intact bacteria. Shown in

Figure 41A is a representative image of protocol 1 applied to human PMNs infected with $N$. gonorrhoeae. These dyes were combined with a lectin, soybean agglutinin, to recognize extracellular $N$. gonorrhoeae, but a fluorescently coupled bacteria-specific antibody could be used as an alternative. Protocol 1 readily reveals the viability of individual bacteria, since exclusion of SYTO9 by propidium iodide makes nonviable bacteria fluoresce red and viable bacteria fluoresce green. However, this protocol cannot be combined with immunofluorescence for host proteins, due to the fluorescence of propidium iodide in two of the four channels available for conventional fluorescence microscopy. Therefore, protocol 2 was developed, which uses SYTOX Green to identify nonviable bacteria and DAPI to detect the total bacterial population. An example of $N$. gonorrhoeae associated with and inside human PMNs and processed using SYTOX Green and DAPI is shown in Figure 41B and an example in combination 
with immunofluorescence is shown in Figure 41C. Besides the availability of two additional fluorescence channels, this protocol is advantageous for use by individuals with red-green colorblindness. However, nonviable bacteria can fluoresce green or bluegreen, depending on the relative intensity of DAPI and SYTOX Green staining and on the image acquisition parameters. One drawback to the use of DAPI is that it can leak from $N$. gonorrhoeae during the course of infection, although this may not be an issue for other bacteria such as Staphylococcus aureus (our unpublished observations). To counteract this issue, infected cells were labeled with DAPI along with SYTOX Green after infection, but the intensity of the nuclear DAPI staining overpowered the fluorescence of $N$. gonorrhoeae (our unpublished observations). In addition to these two protocols, other commercially available dyes could be used to reveal bacterial viability. For instance, carboxyfluorescein succinimidyl ester (CFSE) could label the total bacterial population, and SYTOX Orange or Blue could identify nonviable bacteria. Since the relative efficacy of these procedures may vary with the bacterium and infected cell type, it is important that researchers compare different viability dye combinations in their infection systems.

Currently, we manually examine each image obtained with these protocols to quantify percent internalization, percent viability intracellularly and extracellularly, and colocalization with subcellular markers. While automated computer-based methods can theoretically be used to quantify these parameters, they may be complicated by any nonuniform staining of the bacteria with viability dyes and the strong fluorescence signal of the cell nucleus. Also, the definition of bacterial colocalization with a subcellular marker may need to be optimized for the bacteria, cell type, and marker of interest. 
One limitation of these protocols is the assumption that permeability to propidium iodide or SYTOX Green is a true measure of bacterial death. The molecular weight of propidium iodide and SYTOX Green is 668.4 Da and 600 Da, respectively. These dyes are considered membrane-impermeant and should not access the bacterial cytoplasm unless the bacteria have unrepaired membrane damage. However, the possibility remains that the bacteria enter a state of stasis or even repair their membrane damage and continue to grow at later times. A second limitation is that the protocols do not allow the viability of individual bacteria to be tracked over time. Life Technologies recommends that assessments of bacterial viability with propidium iodide and SYTO9 be performed within 30 minutes after exposure to the dyes, since the bacteria will lose viability the longer they are mounted and imaged by fluorescence microscopy. Infected cells labeled with the DAPI/SYTOX Green dye combination must also be imaged rapidly after mounting (our unpublished observations). Third, the protocols are not compatible with commonly used fixation techniques. Methanol or acetone fixation will permeabilize and kill the bacteria associated with host cells, and aldehyde-based fixatives permeabilize $N$. gonorrhoeae inside human PMNs to propidium iodide and SYTOX Green (unpublished observations). Thus using these protocols requires immediate access to a fluorescence microscope and the ability to complete the protocols in one day. Finally, each of the dyes used in these protocols bind double stranded nucleic acids and bind host nuclear DNA as well as the bacterial DNA (Figure 41). Because fluorescence of the nuclear DNA is significantly brighter than fluorescence of the bacterial DNA, the viability of bacteria which are in proximity to the eukaryotic nucleus cannot be assessed. Confocal laser scanning microscopy and prelabeling bacteria with DAPI helps reduce the nuclear 
fluorescence signal. Fluorescence of nuclear DNA will remain a complication of these protocols until non-DNA-binding bacterial viability dyes are developed.

Despite these limitations, the protocols in this article provide a sensitive way to assess the viability of individual bacteria attached to and inside eukaryotic cells. Colony count or gentamicin-protection assays have been the backbone of microbial pathogenesis research. However, they provide an assessment of bacterial survival across a potentially heterogeneous population. In contrast, the viability dye protocols described here allow the integrity of individual bacteria to be examined. Therefore, researchers can gather data regarding the fates of extracellular vs. intracellular bacteria, bacteria in different phagosomal types, or bacteria in a phagosome $v s$. the cytoplasm. Thin section transmission electron microscopy has often been used to examine the viability of individual bacteria. While bacteria that appear electron-lucent or no longer intact by TEM are clearly nonviable, it is more difficult to assess whether bacteria that retain some electron density in their cytoplasm are actually intact. Additionally, it can take many hours of electron microscope time to acquire enough micrographs to analyze, while many low-power fields can be acquired from cells exposed to fluorescence-based viability dyes, even in the short time afforded for image acquisition. While the provided protocols were optimized with $N$. gonorrhoeae and primary human PMNs, they can be adapted to assess the viability of many species of gram-negative and gram-positive bacteria in a variety of cell types, including all phagocytic and nonphagocytic eukaryotic cells (Johnson et al., 2013b, Criss et al., 2009, Chow et al., 2010, Kaplan et al., 2006, Thulin et al., 2006, Kubica et al., 2008, Swords et al., 2006, Tamilselvam et al., 2006, Martinez et al., 2009, Botha et al., 2012, Allen et al., 2000, Smith et al., 2012). Bacterial viability dyes provide 
a powerful experimental tool to examine mechanisms of bacterial pathogenesis in eukaryotic cells and the ability of eukaryotic cells to control bacterial infection. In the future, these protocols are likely to become even more useful with better tools for automated image acquisition and processing, along with the development of bacterial viability dyes that are compatible with aldehyde fixation and specific for prokaryotes. 
5.5 Figures for Appendix A

Syto9

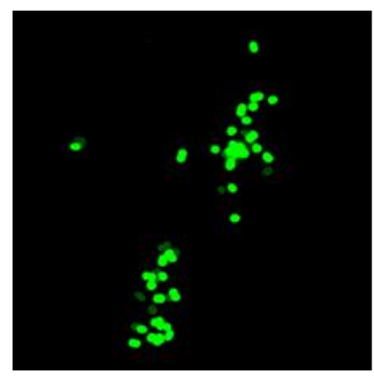

Propidium lodide SBA-Alexa647
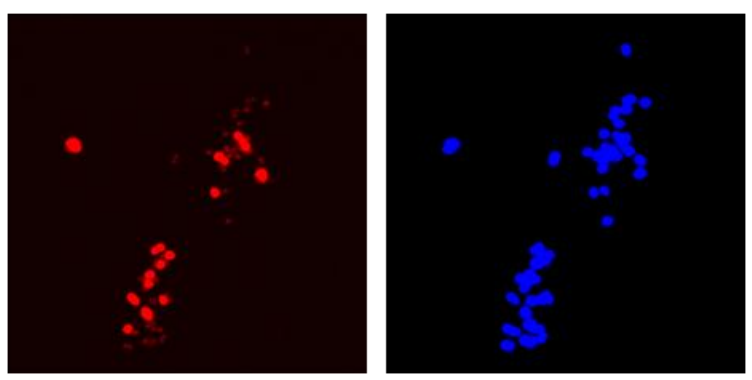

Merge

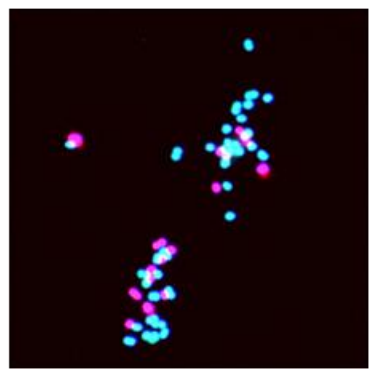

Figure 38: Lectin labels viable and nonviable Gc

A mix of viable and nonviable $N$. gonorrhoeae was exposed to the Alexa Fluor 647coupled soybean lectin, propidium iodide, and SYTO9 according to protocol 1, in the absence of host cells. All bacteria are accessible to the lectin and fluoresce blue.

Nonviable bacteria appear blue + red and nonviable bacteria appear blue + green . 

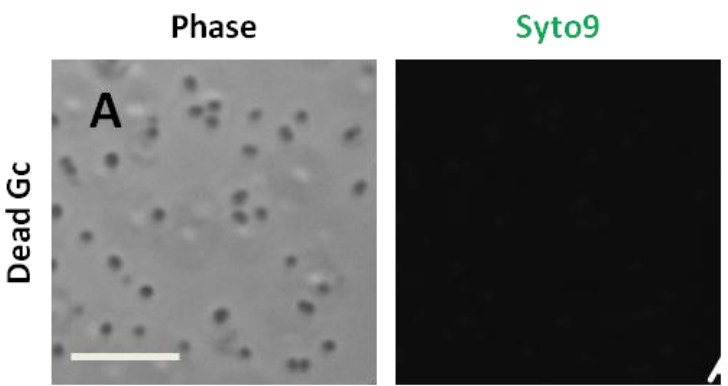

Propidium Iodide
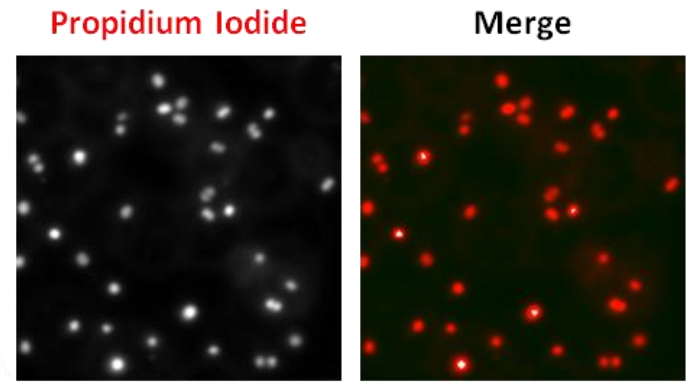
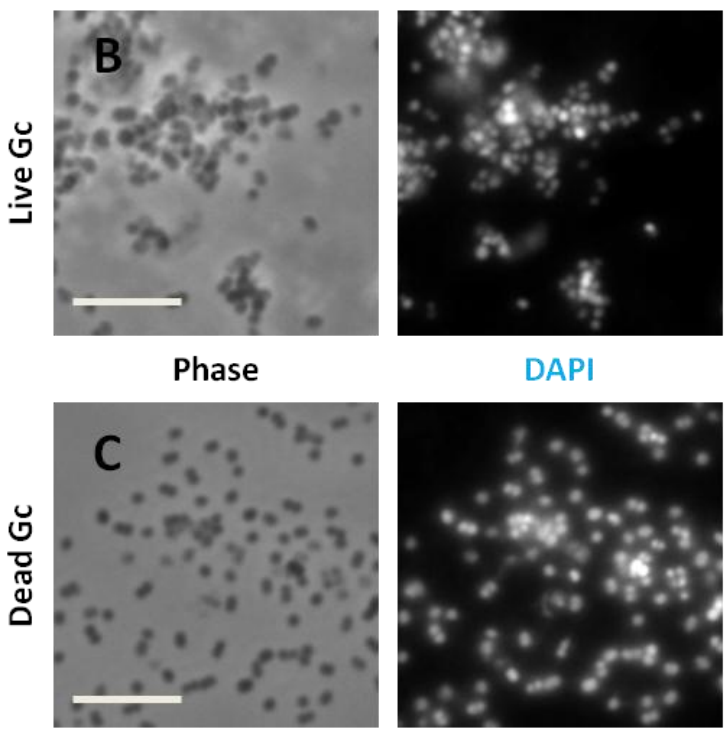

DAPI
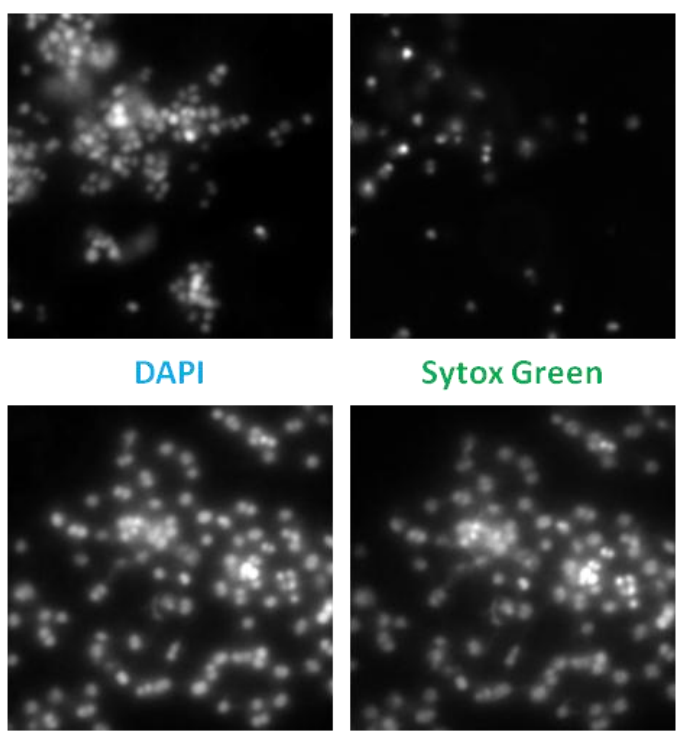

Sytox Green
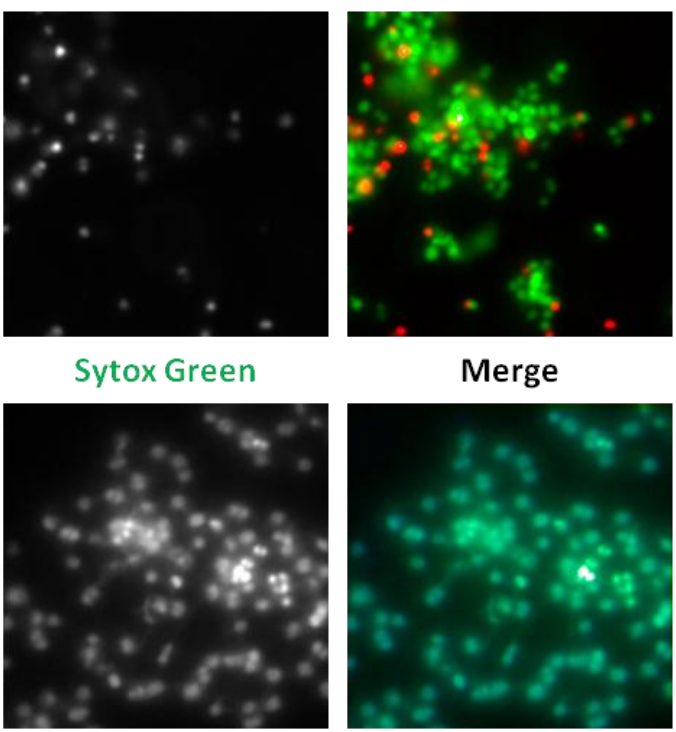

Merge
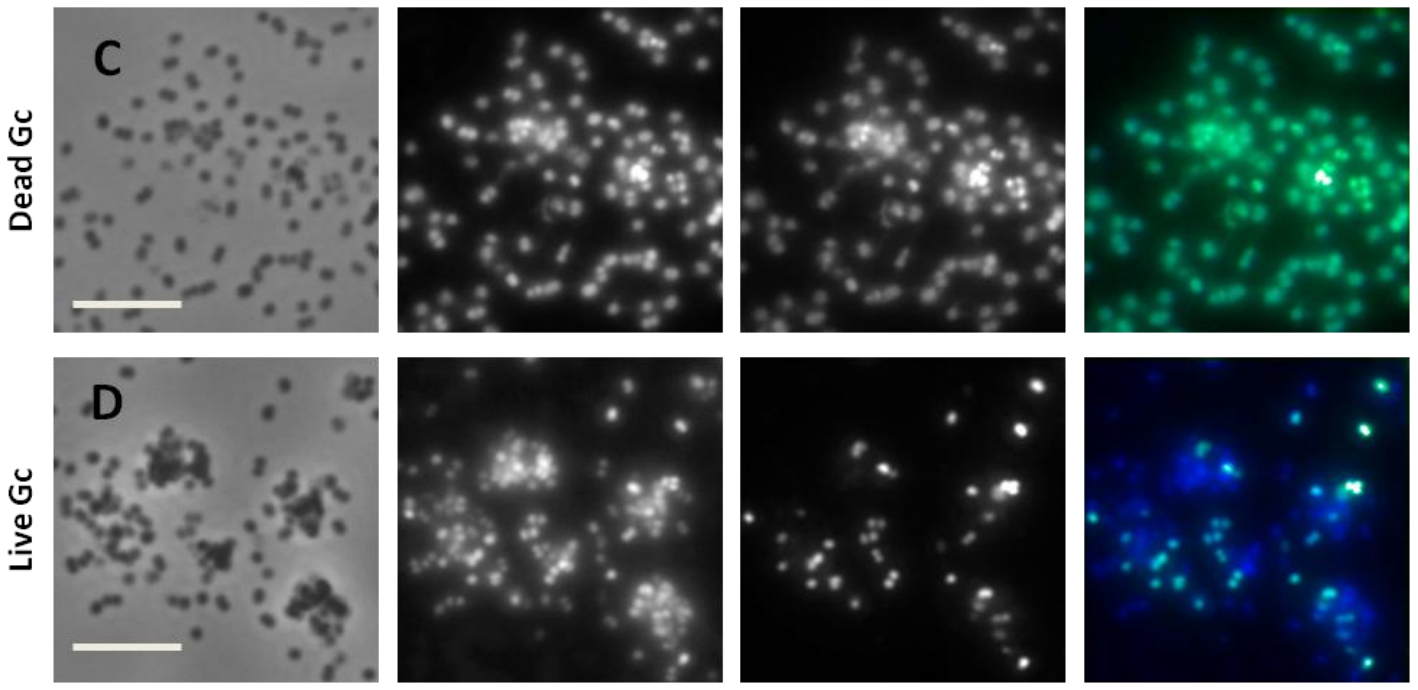

Figure 39: DNA dyes distinguish viable and nonviable bacteria

(A-B) Mid-logarithmic phase $N$. gonorrhoeae was exposed to propidium iodide and SYTO9 as in protocol 1, with (A) or without (B) isopropanol treatment to kill the bacteria. Nonviable bacteria are accessible to propidium iodide and appear red. Viable bacteria are stained with SYTO9 and appear green. (C-D) Mid-logarithmic phase $N$. gonorrhoeae was exposed to DAPI and SYTOX Green as in protocol 2, with (C) or without (D) isopropanol treatment to kill the bacteria. Nonviable bacteria are accessible to DAPI and SYTOX Green and appear blue + green. Viable bacteria are stained with DAPI only and appear blue only. 


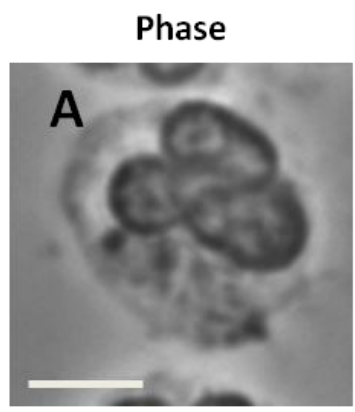

Phase

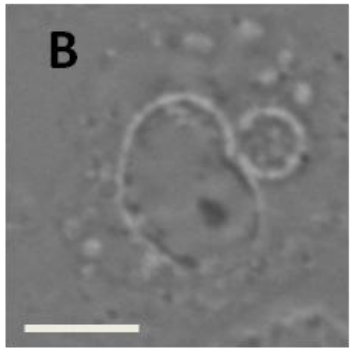

Syto9

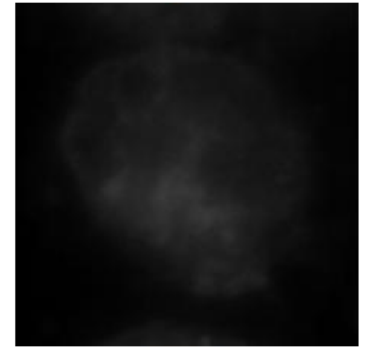

DAPI

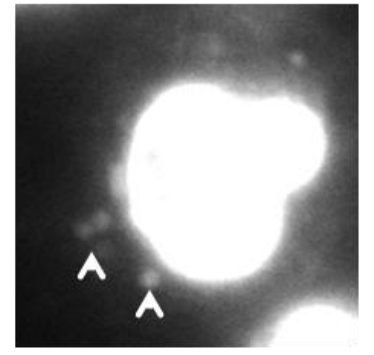

Propidium Iodide

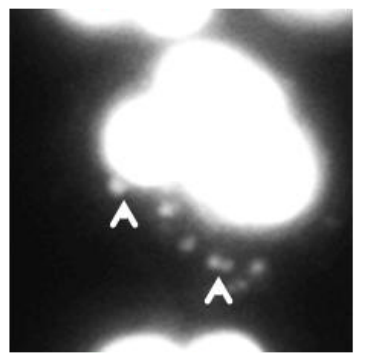

Sytox Green

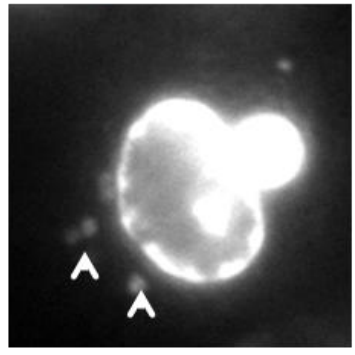

Merge

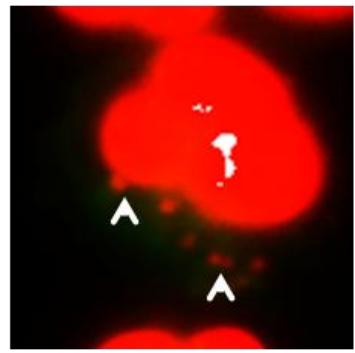

Merge

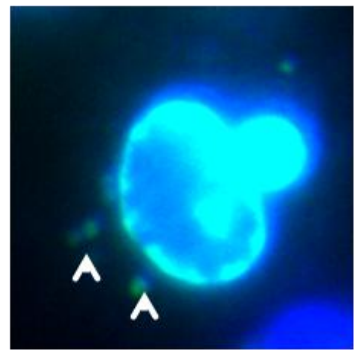

\section{Figure 40: DNA dyes identify nonviable bacteria in host cells}

(A-B) Mid-logarithmic phase $N$. gonorrhoeae was exposed to propidium iodide and PMNs were allowed to bind and internalize isopropanol-killed $N$. gonorrhoeae, then exposed to propidium iodide and SYTO9 according to protocol 1. Nonviable bacteria are stained with propidium iodide and appear red. As all bacteria are nonviable, no SYTO9 positive, green bacteria are detected. (B) PMNs were allowed to bind and internalize isopropanol-killed $N$. gonorrhoeae, then exposed to DAPI and SYTOX Green according to protocol 2. Nonviable bacteria are stained with DAPI and SYTOX Green and appear blue + green. As all bacteria are nonviable, no DAPI only, blue only bacteria are detected. $\mathrm{N}$ labels the PMN nucleus. Scale bar $=5 \mu \mathrm{m}$. Arrowheads indicate some of the nonviable bacteria. 


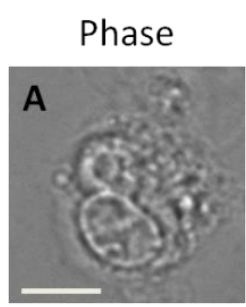

Phase

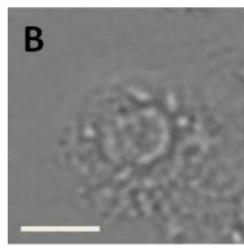

Phase

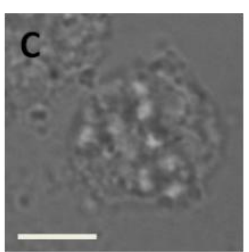

SBA-Alexa647

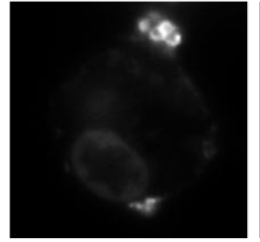

SBA-Alexa647

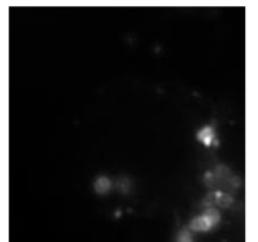

SBA-Alexa647

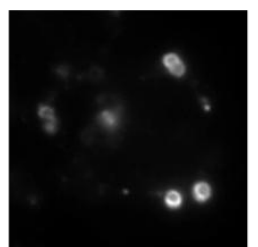

SYTO9

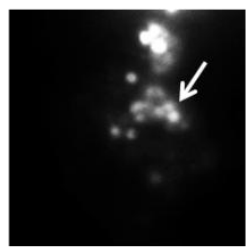

DAPI

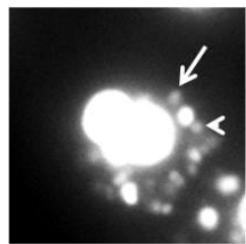

DAPI

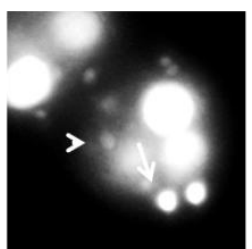

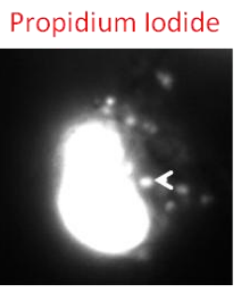

Sytox Green

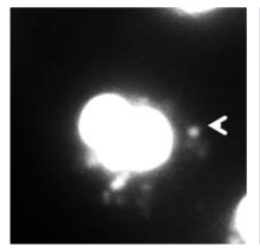

Sytox Green

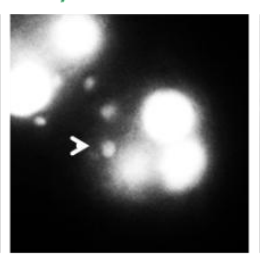

Merge

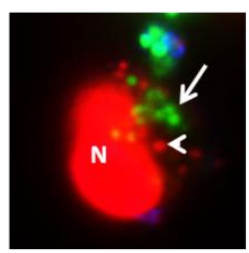

Merge

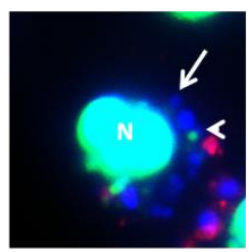

CD63

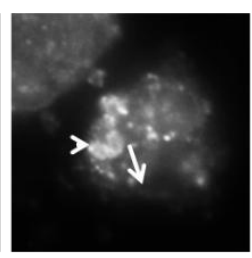

Merge

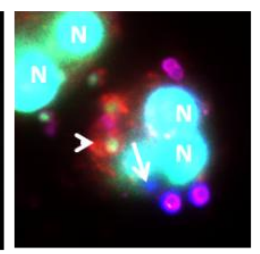

\section{Figure 41: DNA dyes distinguish viable and nonviable bacteria in host cells}

(A) N. gonorrhoeae-infected human PMNs were exposed to Alexa Fluor 647-coupled soybean lectin (false-colored blue), then permeabilized with saponin in the presence of the viability dyes propidium iodide (red), to label dead bacteria, and SYTO9 (green), to counterstain live bacteria. External nonviable bacteria appear blue + red, internal nonviable bacteria appear red only, external viable bacteria appear blue + green, and internal viable bacteria appear green only. (B) N. gonorrhoeae was labeled with DAPI (blue), then presented to PMNs. Infected cells were then exposed to Alexa Fluor 647coupled soybean lectin (false-colored red), then permeabilized with saponin in the presence of SYTOX Green (green) to label dead bacteria. External nonviable bacteria appear red + green, internal nonviable bacteria appear green + blue, external viable bacteria appear red + blue, and internal viable bacteria appear blue only. (C) $N$. gonorrhoeae-infected PMNs were processed as in B, except an Alexa Fluor 555-coupled antibody against the PMN primary granule protein CD63 (red) was added at the time of permeabilization. The Alexa Fluor 647-soybean lectin staining was false-colored purple. External nonviable bacteria appear purple + green, internal nonviable bacteria appear green only, external viable bacteria appear purple + blue, internal viable bacteria appear blue only, and CD63 staining appears red. The inset shows the CD63 fluorescence of the area of the image indicated with the white square. In A-C, N labels the PMN nucleus. Scale bar $=5 \mu \mathrm{m}$. Arrowheads indicate nonviable bacteria and arrows indicate viable bacteria. 


\section{Appendix B: Investigation of Gc Replication inside primary human PMNS}

\subsection{Introduction}

During gonorrheal infection there are abundant PMNs present; however, viable Gc can be cultured from patient exudates, which is suggestive of Gc survival (Wiesner et $a l ., 1980)$. Further investigation of these exudates also revealed the presence of abundant PMNs with associated and internalized Gc (Ovcinnikov et al., 1971, Apicella et al., 1996, King et al., 1978a, Farzadegan et al., 1975). In vitro studies demonstrate approximately $50 \%$ of internalized Gc remain viable in PMNs (Simons et al., 2005, Criss et al., 2009). Most recently the studies presented in this thesis demonstrate that phagosomes containing Opa- Gc delay fusion with primary granules, allowing Gc to avoid exposure to PMN nonoxidative antimicrobial components in order to survive intracellularly (Johnson et al., 2013b). Collectively this data present strong evidence that Gc is capable of surviving inside primary human PMNs.

The benefit to Gc survival inside PMNs is often questioned due to the short lifespan of PMNs. However, the Apicella group has demonstrated that PMNs infected with Gc delay apoptosis(Simons et al., 2006). Therefore, Gc survival and potentially replication inside PMNs could serve as an important contributor to Gc pathogenesis. Several lines of evidence are suggestive of Gc replication inside these hostile antimicrobial cells. Seminal studies demonstrated that Gc inside PMNs were sensitive to penicillin, which is capable of killing only replicating bacteria. Experiments using impermeable antimicrobial agents to kill extracellular bacteria demonstrated there is an increase in viable colony forming units (CFU) over time (Veale et al., 1976, Veale et al., 
1979, Casey et al., 1980). Finally, increases in the number of viable Gc inside PMNs over time have been observed, using bacterial viability dyes and electron microscopy (Criss et al., 2009, Simons et al., 2005). However, there are key limitations to these studies.

Studies using antimicrobial agents to kill extracellular Gc are dependent on the sensitivity of Gc to the antimicrobial agents as well as the in ability of these antimicrobial agents to be macropinocytosed by PMNs. Studies using imaging of viable Gc inside PMNs were conducted in assay conditions where the extracellular population of Gc was not killed. Therefore, increases in intracellular Gc could instead have been due to continued phagocytosis of extracellular Gc by PMNs.

The aim of this study was to seek direct evidence for Gc replication inside primary human PMNs. I initially conducted an assay where infected PMNs were placed in media that was not supportive of extracellular Gc survival, and CFU counts were measured over time. Results from this assay were suggestive of Gc intracellular replication. Therefore, I proposed using 5-ethynyl-2'-deosyuridine (EdU), a nucleoside analog of thymidine which would be incorporated into bacterial DNA during replication. PMNs are terminally differentiated cells, so EdU would not label PMNs. While EdU was successful at selectively labeling replicating Gc in culture, initial attempts to identify intracellular replicating Gc in PMNs were unsuccessful.

\subsection{Materials and Methods}

\subsubsection{Bacterial strains and growth conditions}

The Gc of strain FA1090 used in this study constitutively expresses the pilin variant 181-S2 due to mutation of the guanine quartet sequence upstream of pilE (Cahoon et al., 
2009). The strain defined as Opa- has in-frame deletions of the genes encoding the 11 opacity-associated (Opa) proteins (A-K)(Ball et al., 2013). Piliated, Opa-negative Gc was routinely grown on Gonococcal Medium Base (Difco) plus Kellogg's supplements (Kellogg et al., 1963) for $20 \mathrm{~h}$ at $37^{\circ} \mathrm{C}$ in $5 \% \mathrm{CO}_{2}$. Gc was grown to exponential phase via successive rounds of bacterial growth in rich liquid medium as described (Criss et al., 2008). Viable, exponential phase Gc was used to infect primary human PMNs. To kill Gc prior to PMN exposure, Gc was incubated for 5 min at RT with isopropanol.

\subsubsection{Bacterial Survival Assay}

Adherent PMNs were infected with exponential phase Gc and Gc survival was assessed by cell lysis and plating for viable CFU as previously described(Stohl et al., 2005), with the following modification. After 1 hour of infection in Roswell Park Memorial Institute media (RPMI) $+10 \%$ Fetal Bovine Serum (FBS), the media was removed and replaced with Hank's Buffered Salt Solution (HBSS) containing 1\% BSA and 0.15\% Dextrose for the remaining 5 hours of infection.

\subsubsection{EdU labeling of exponential phase Gc}

Gc were grown to exponential phase using a modification of the previously described protocol (Stohl et al., 2005, Criss et al., 2008). Gc cultured for 9-10 h on GCB were suspended at $\mathrm{OD}_{550}=0.07$ in gonococcal liquid medium containing Kellogg's supplements and $0.042 \% \mathrm{Na}_{2} \mathrm{HCO}_{3}(\mathrm{GCBL})$ and grown at $30^{\circ} \mathrm{C}$ with rotation for 14 $16 \mathrm{~h}$. The cultures were diluted into fresh GCBL and grown at $37^{\circ} \mathrm{C}$ to $\mathrm{OD}_{550}=0.5-0.6$. Cultures were then diluted to $\mathrm{OD}_{550}=0.07$ and grown at $37^{\circ} \mathrm{C}$ for $1 \mathrm{~h}$. Cultures were grown an additional $2 \mathrm{~h}$ at $37^{\circ} \mathrm{C}$ to mid-logarithmic phase in the presence of $20 \mu \mathrm{M} \mathrm{EdU}$ 
(Invitrogen). The cfu per $\mathrm{ml}$ present in each culture was estimated from a previously determined growth curve and validated by serial dilution and plate count. Bacteria were pelleted for $3 \mathrm{~min}$ at $10,000 \mathrm{~g}$ and washed in $1 \mathrm{XPBS}$ containing $3 \% \mathrm{BSA}$. In order to detect EdU, bacteria were incubated in the Click it Rxn Cocktail (Invitrogen) for $30 \mathrm{~min}$ at room temperature in the dark. Bacteria were washed in 1XPBS containing 3\% BSA. Bacteria were mounted on slides and imaged immediately. Images were acquired on a Nikon Eclipse E800 with Hamamatsu Orca-ER digital camera using Openlab software and processed in Adobe Photoshop CS5.

\subsubsection{EdU labeling of Gc inside PMNs}

$10^{6} \mathrm{PMNs}$ were added per serum coated coverslip and incubated for $1 \mathrm{~h}$ at $37^{\circ} \mathrm{C}, 5 \% \mathrm{CO}_{2}$ to promote PMN attachment as previously described (Johnson et al., 2013b). PMNs were infected with viable, exponential-phase Gc or isopropanol-killed Gc at a multiplicity of infection of 1-5 bacterial colony forming units (CFU) per PMN as described in(Criss et al., 2009). After 1 hour of infection the RPMI + 10\% FBS media was replaced with HBSS containing $1 \%$ BSA and $0.15 \%$ Dextrose. After an additional 1 hour of infection at $37^{\circ} \mathrm{C}$, EdU was added to each coverslip to a final concentration of $20 \mu \mathrm{M}$ and incubated for 4 hours at $37^{\circ} \mathrm{C}$. Cells were fixed with $4 \%$ PFA. External and internal Gc were distinguished using a polyclonal rabbit anti-Gc antibody as previously described (Johnson et al., 2013b). Cells were permeabilized with $0.5 \%$ Triton X-100 in $1 \mathrm{X}$ PBS followed by an incubation in the Click it Rxn Cocktail (Invitrogen) for $30 \mathrm{~min}$ at room temperature in the dark. Cells were washed with 1XPBS containing 3\% BSA and coverslips were

mounted using Flouromount $\mathrm{G}$ (Southern Biotech) with $2.5 \mathrm{mg} \mathrm{ml}^{-1}$ propyl gallate (Acros 
Organics). Images were acquired on a Nikon Eclipse E800 with Hamamatsu Orca-ER digital camera using Openlab software and processed in Adobe Photoshop CS5.

\subsection{Results}

In order to determine if Gc replicates intracellularly in PMNs, PMNs were infected with Gc in HBSS media, which prevents extracellular Gc replication. At 1, 2, 4, and 6 hours, PMNs were lysed, and bacteria were enumerated from cell lysates as CFU after overnight growth. Gc survival in the presence of PMNs was compared to Gc survival in RPMI+10\% FBS alone or in HBSS alone over 6 hours. We observed that Gc grew in RPMI $+10 \%$ FBS for the first 3 hours and then declined, which we anticipate being due to a limitation in nutrients over the 6 hour time course. In contrast, Gc in HBSS alone exhibited no significant increase in CFU over time. We observed that in the presence of PMNs in HBSS, Gc survival increased over time. (Figure 42). These results indirectly suggest that Gc replicates intracellularly in PMNs. However, an alternative explanation is that PMNs release nutrients into the media to support extracellular Gc growth. Therefore, we continued our investigation and designed an immunofluorescence assay to directly visualize Gc replication inside PMNs.

In order to monitor Gc replication by immunofluorescence, we utilized EdU, a nucleoside analog of thymidine. During bacterial replication, EdU is incorporated into the bacterial DNA and then detected using a "click it" reaction cocktail. As an initial control, viable Gc were grown and nonviable Gc were rotated in media containing EdU. We observed that EdU selectively labeled viable replicating Gc (Figure 43). In order to determine if Gc were replicating inside PMNs, PMNs were infected with viable Gc or 
exposed to nonviable Gc. Following 2 hours of infection EdU was added to the media and incubated for an additional 4 hours. Following fixation, intracellular and extracellular Gc were discriminated from each other by incubation with a polyclonal rabbit anti-Gc antibody before and after PMN permeabilization. EdU incorporation was then detected using the click it reaction cocktail. We observed from these initial experiments that EdU labeled not only the bacteria in PMNs infected with viable Gc, but also the nonviable Gc that were presented to PMNs (Figure 44). From these data we conclude that this experimental design was not capable of using EdU to selectively identify viable replicating Gc in the context of PMNs. However, modification of this protocol may lead to an approach which would allow for detection of replicating Gc in PMNs.

\subsection{Discussion}

Gc survival in PMNs is well established (Johnson et al., 2013b, Ovcinnikov et al., 1971, Apicella et al., 1996, King et al., 1978a, Farzadegan et al., 1975, Criss et al., 2009, Simons et al., 2005); however, Gc replication in PMNs has not been definitively demonstrated. In order to demonstrate Gc replication in PMNs, we initially monitored Gc survival in PMNs over 6 hours by plating for CFU. This assay was conducted in media which is not conducive to Gc replication, thereby preventing external bacterial replication. We observed that Gc survival increased over 6 hours, suggesting Gc were replicating inside PMNs. Therefore, we designed an immunofluorescence assay to visually monitor Gc replication inside PMNs. Using EdU, we were able to identify replicating Gc in culture, but we were unable to selectively identify replicating Gc in the context of PMNs. This precluded us from establishing if Gc replicate intracellularly in PMNs. 
Our initial plating assay indicated that Gc were replicating intracellularly in PMNs. However, the observed increase in viable Gc over time in this assay may be a result of external Gc growth. HBSS does not normally support Gc growth, but the release of nutrients from Gc activated or dying PMNs may be sufficient to supplement the media and allow for external Gc growth. Therefore, it is difficult to conclude solely from this assay that Gc are in fact replicating inside PMNs. Future experiments could address this possibility by infecting PMNs or lysing PMNs in HBSS. The media could then be recovered and sterile filtered to remove any remaining Gc and/or cell debris. Gc could then be added to the media and survival evaluated by plating viable CFU. If Gc are still unable to survive in the HBSS media supplemented with components released by Gc infected or lysed PMNs, this would be a strong indicator that Gc are replicating intracellularly in PMNs.

In order to accomplish direct visualization of Gc replicating inside PMNs, we selected to use EdU. EdU is incorporated into DNA during DNA synthesis and therefore should label all replicating Gc. We selected EdU because it is easy to detect using the click it reaction cocktail which results in incorporated EdU being fluorescent. In contrast to CFSE, a fluorescent dye which dilutes out as bacteria replicate, EdU is incorporated as bacteria replicate and fluorescence increases. This is advantageous since Gc is only 0.5$1 \mu \mathrm{m}$ and as such a dilution in signal may be difficult to detect. Unfortunately, EdU was only able to detect replicating Gc in culture and not selectively identify replicating Gc in PMNs. We observed that the total Gc population was labeled with EdU regardless if PMNs were infected with viable Gc or exposed to nonviable Gc. There are three explanations for this result. First, the immunofluorescence staining protocol used to 
discriminate internal and external Gc is not compatible with EdU detection. Second, the secondary antibody coupled to Alexa Fluor 555 which was used to detect the total Gc population and EdU Alexa Fluor 488 have spectral overlap resulting in the total population of Gc appearing positive for EdU staining. Third, EdU incorporation into or detection of replicating Gc is altered by PMN granule components which are present in the Gc containing phagosome. Additional troubleshooting to address these potential problems may yield an experimental design to allow for EdU detection of replicating Gc.

During gonorrheal infection, the ability of Gc to replicate inside PMNs would be advantageous for disease persistence, progression, and transmission (Johnson et al., 2011, Criss et al., 2012). Abundant PMNs are recruited to the site of Gc infection (Edwards et al., 2004). The ability of Gc to not only survive but also replicate inside PMNs would provide a replication niche which is protected from complement and antibody mediated killing. Protection from host mediated killing may prolong infection and assist in dissemination. Disseminated infection leads to several complications including arthritisdermatitis syndrome, endocarditis, and meningitis (Wiesner et al., 1980). PMNs may traffic viable and replicating Gc across the epithelial barrier more effectively leading to increased dissemination. Finally, patient exudates contain numerous PMNs with associated and internalized Gc (Ovcinnikov et al., 1971, Apicella et al., 1996, King et al., 1978a, Farzadegan et al., 1975). Therefore, PMNs containing viable and replicating Gc in the exudates could increase transmission between individuals. The ability of Gc to replicate inside PMNs remains an important research question in the field. Further investigation of if, when, and where Gc replicate inside PMNs may provide greater 
understanding of Gc pathogenesis and lead to the development of novel treatment options. 


\subsection{Figures for Appendix B}

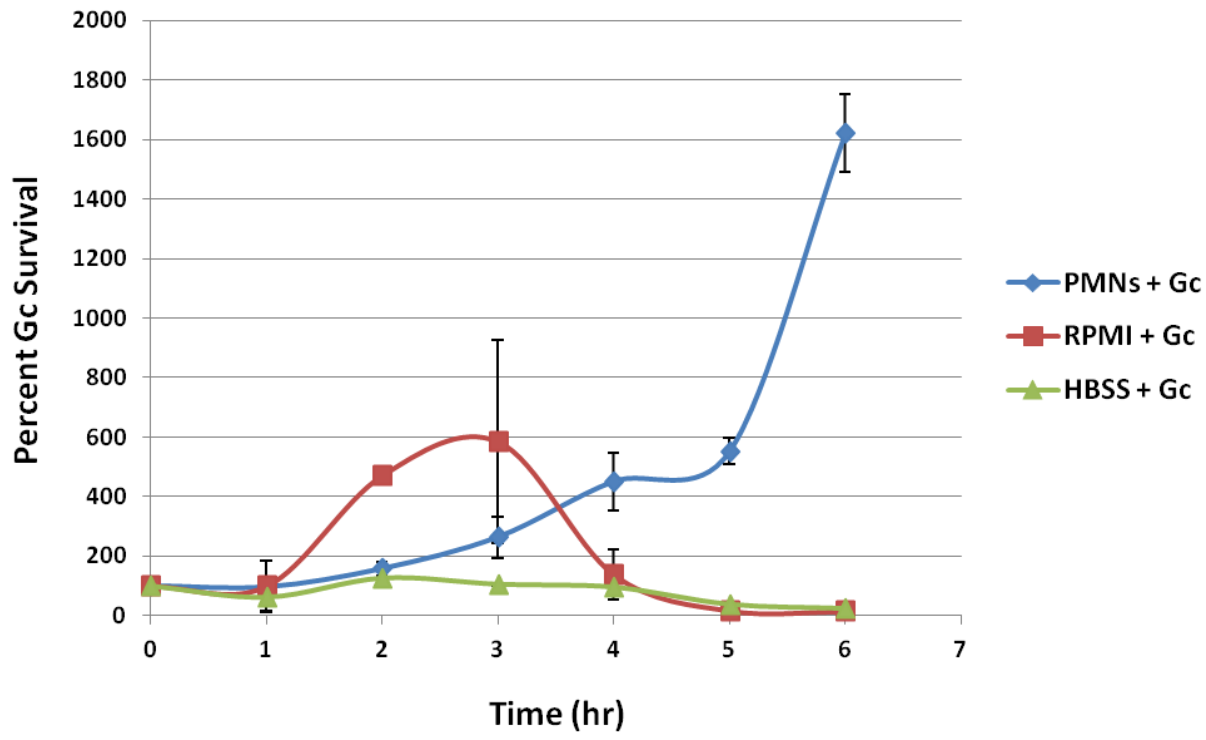

\section{Figure 42: Gc replication inside PMNs}

Gc survival in PMNs was compared to Gc survival in RPMI $+10 \%$ FBS or HBSS.

Survival of Gc was plotted over time. Bacteria survival equals the CFU enumerated from PMN lysates or in media alone at each time point divided by the CFU present at 0 min. 


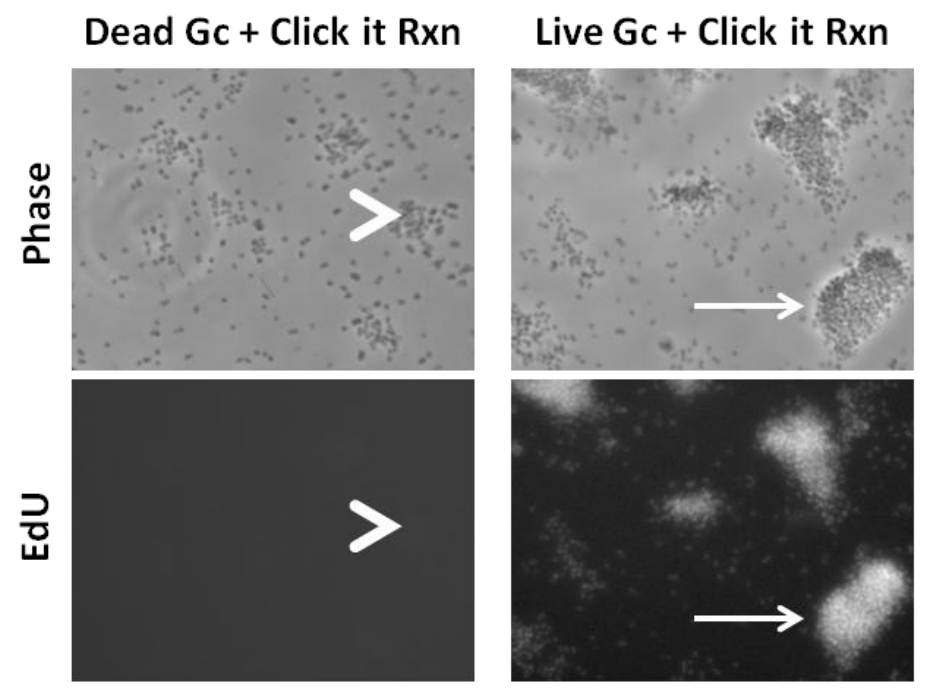

Figure 43: EdU detection of replicating Gc

Live replicating Gc and Dead Gc were incubated with EdU for 2hrs. EdU labeled only live replicating Gc. Arrow indicate live Gc labeled with EdU and arrowheads indicate dead Gc which are not labeled with EdU. 

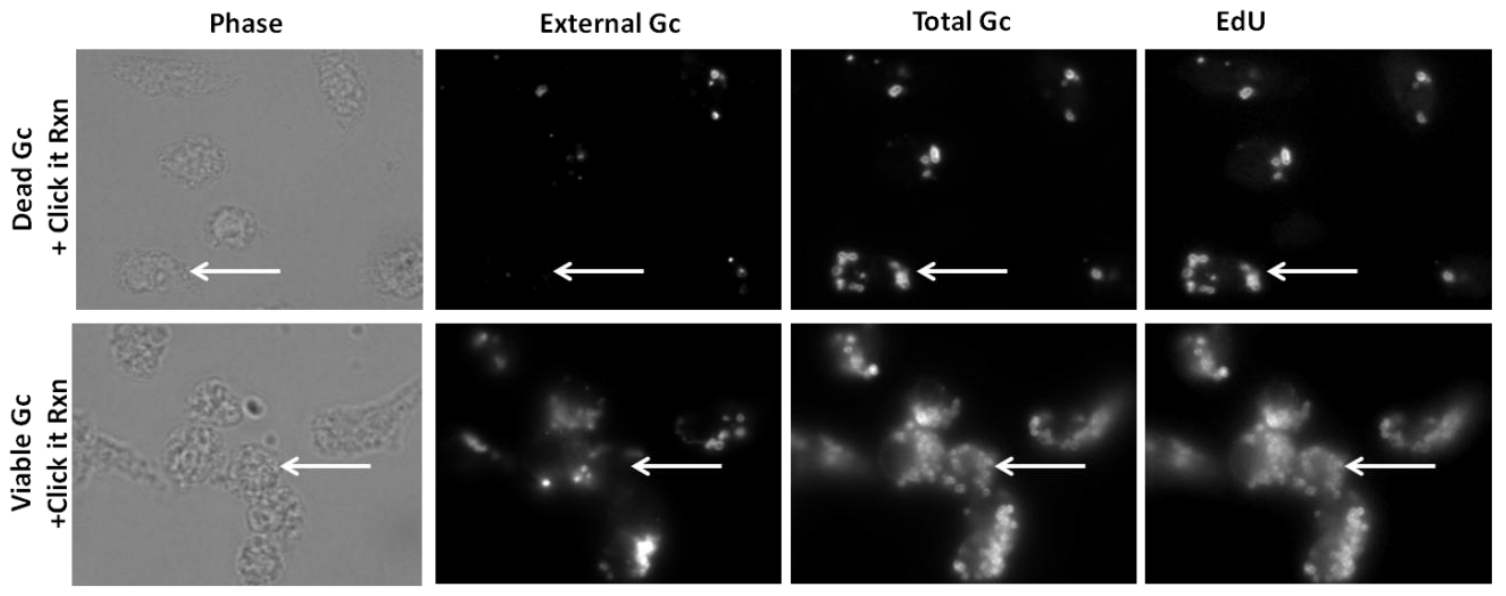

Figure 44: EdU detection of replicating Gc in PMNs

PMNs were infected with live replicating Gc or exposed to dead Gc for 6 hrs. Internal and external Gc were discriminated via immunofluorescence and EdU incorporation detected using the click it reaction cocktail. Arrows indicate internal Gc labeled with EdU. 


\section{Appendix C: Role of pili in Gc survival in PMNs}

\subsection{Introduction}

Gc possess a type IV pilus which is important for immune evasion and mediating interactions with host cells during gonorrheal infection. Isolates recovered from naturally infected males and females and experimentally infected males are uniformly piliated (Kellogg et al., 1963, Kellogg et al., 1968). Results from experimental infection of male volunteers with Gc indicate that infection with piliated Gc results in symptomatic infection while nonpiliated Gc infection asymptomatic or yields a less severe discharge (Kellogg et al., 1963, Kellogg et al., 1968). The type IV pilus aids in Gc in escaping immune detection due to phase variation and antigenic variation. Extensive antigenic variation of PilE has been observed in experimental infection of male volunteers (Seifert et al., 1994, Wright et al., 1994, Hamrick et al., 2001, Swanson et al., 1987). Antigenic variation occurs when the major pilin subunit gene, pilE, recombines with one of several potential silent pilin gene cassettes (Cahoon et al., 2011). Antigenic variation is a highly effective mechanism for evading antibody mediated detection. A vaccine against the Gc pilus effectively identified homologous but not heterologous pili. In experimental trials the vaccine did not protect volunteers from Gc infection (Boslego et al., 1991). Gc pilus phase variation additionally assists in immune evasion and results from two mechanisms. First, pili are phase off when the minor pilin subunit pilC is not expressed due to slipped strand mispairing at a mononucleotide tract in the coding regions of two pilC genes. PilC counteracts pilT mediated pilus retraction; therefore, in the absence of pilC pili are retracted and Gc are nonpiliated (Morand et al., 2004). Second, pili can be phase off due to recombination at pilE, resulting in a truncated or nonfunctional pilin subunit. The 
combination of phase and antigenic variation likely contributes to an inability of the host to develop a protective immune response to Gc infection.

In order to establish infection Gc use type IV pili to initially attach to epithelial cells. PilC is associated with the outer membrane but can also be recovered from purified pili (Rudel et al., 1995, Rahman et al., 1997). PilC has been shown to be localized to the pilus tip and has been identified as the major pilin adhesin (Rudel et al., 1995, Nassif et al., 1994). These results have not been recapitulated by other researches in the field. CD46 was first identified as the pilus receptors on epithelial cells (Kallstrom et al., 1997, Kallstrom et al., 2001). However, it is located on the basolateral surface of epithelial cells and this may limit Gc access to CD46 (Simpson et al., 1997). Additionally, there is evidence to suggest there is no correlation between CD46 expression and Gc internalization, indicating CD46 may not serve as a pili receptor (Tobiason et al., 2001, Kirchner et al., 2005a). Recent research indicates that cooperative binding of pili with PorB and lipooligosaccharide to complement receptor 3 can mediate internalization into epithelial cells (Edwards et al., 2005). Regardless of the host receptor, it is clear that an important function of pili is to mediate Gc adherence.

Pili have also been observed to modulate Gc interactions with PMNs. Early studies indicate that type $1 \mathrm{Gc}$ (piliated) resist phagocytosis by PMNs and survive better over time while type 4 Gc (non-piliated) are rapidly phagocytosed and killed (Swanson, 1974, Dilworth, 1975, Thomas, 1973). These initial experiments did not account for other differences between type 1 and type 4 Gc such as Opa protein expression. Experiments which take into account Opa protein expression in addition to piliation state 
indicate that pili have little effect on the degree to which Gc adhere and are killed by PMNs (Virji, 1986). Recent studies have observed that Gc can interact with the PMN uropod via pili in a PilC-dependent manner in order to prevent internalization (Soderholm et al., 2011). Additionally, the NGO1686 metalloprotease which affects Gc piliation has been observed to provide defense against oxidative damage and PMN killing (Stohl et al., 2005, Criss et al., 2009, Stohl et al., 2013).In support of pili providing a survival advantage in PMNs, this study demonstrated that deletion of pilQ, the gene encoding the protein pore through which pili assembles, results in a Gc survival defect in PMNs (Stohl et al., 2013). These studies provide evidence that pili are important for Gc survival in PMNs and emphasize the need for additional studies to fully characterize the role of pili in mediating Gc internalization by PMNs and Gc defense against both oxidative and nonoxidative PMN killing.

Previous investigation of Type IV pili utilized a Gc strain FA1090 containing a spontaneous $950 \mathrm{bp}$ deletion of pilE and flanking regions. Although this mutant was observed to have a survival defect in PMNs, there were two concerns with this result. First, this defect in survival could be due to loss of the regions surrounding pilE, rather than pilE itself. Second, the spontaneous pilE deletion is in a Gc background which has the ability to phase vary each of the 11 opa genes, which may confound experimental results due to the ability of Opa to affect PMN activation. Therefore, in order to investigate the role of Type IV pili in Ge survival in PMNs, we used the Gc AopaA-K background to construct an in-frame deletion of the major pilin subunit gene, pilE. Deletion of pilE resulted in Gc with a non-piliated colony morphology. Additionally, we confirmed the in frame deletion of pilE via PCR and Southern blot. In contrast to 
previous data, we observed Gc with an in frame deletion of pilE did not exhibit a significant difference in survival in PMNs compared to $\triangle o p a A-K \mathrm{Gc}$. Surprised by this result, we generated three additional mutants: an in-frame deletion of pilE in the FA1090 parent background, a backcross of the spontaneous deletion into the variable FA1090 parent background, and introduction of the spontaneous deletion into the $\Delta \mathrm{OpaA}-\mathrm{K}$ background. These mutants did not display significantly reduced survival in PMNs compared to $\triangle o p a A-K$ Gc either, suggesting pilE does not provide a Gc survival advantage in PMNs. The reasons for this discrepancy with previous findings are unclear.

\subsection{Material and Methods}

\subsubsection{Bacterial strains and growth conditions}

The Gc of strain FA1090 used in this study constitutively expresses the pilin variant 181-S2 due to mutation of the guanine quartet sequence upstream of pilE (Cahoon et al., 2009). The strain defined as $\mathrm{Opa} \Delta \mathrm{A}-\mathrm{K}$ has in-frame deletions of the genes encoding the 11 opacity-associated (Opa) proteins (A-K) (Ball et al., 2013). The strain defined as FA1090 spont $\triangle p i l E$ has a 950bp spontaneous deletion in FA1090 1-81-S2 which includes the gene for pilE, the major pilin subunit and the surrounding region. The strains defined as Opa $\triangle \mathrm{A}-\mathrm{K} \triangle p i l E$ and FA1090 $\Delta p i l E$ have an in frame deletion of the pilE gene in the

Opa $\triangle \mathrm{A}-\mathrm{K}$ and FA1090 parent backgrounds. The strains defined as Opa $\Delta \mathrm{A}-\mathrm{K}$ large $\Delta p i l E$ and FA1090 large ApilE have a deletion of 950bp which is lost in the FA1090 spont 1 pilE strain in the Opa $\triangle \mathrm{A}-\mathrm{K}$ and FA1090 parent backgrounds. Piliated, Opa-negative Gc was routinely grown on Gonococcal Medium Base (Difco) plus Kellogg's supplements (Kellogg et al., 1963) for $20 \mathrm{~h}$ at $37^{\circ} \mathrm{C}$ in $5 \% \mathrm{CO}_{2}$. Gc was grown to exponential phase 
via successive rounds of bacterial growth in rich liquid medium as described (Criss et al., 2008). Viable, exponential phase Gc was used to infect primary human PMNs.

\subsubsection{In frame deletion of $\Delta$ pilE}

In-frame deletions of the pilE gene were created using overlap extension PCR(Heckman et al., 2007). Approximately $0.9 \mathrm{~kb}$ of sequence upstream (fragment A) and $0.6 \mathrm{~kb}$ of sequence downstream (fragment B) of pilE was amplified by PCR from FA1090 1-81-S2 P_nv $\triangle O p a B E G K$ genomic DNA. Table 2 contains primer sequences and Table 3 contains PCR conditions. The deletions were designed to replace most of the pilE open reading frame $(\mathrm{ORF})$ with a PvuII restriction site. $\triangle \mathrm{OpaBEGK}, \triangle \mathrm{OpaA}-\mathrm{K}$, and FA1090 Gc were transformed with the overlap extension $1.5 \mathrm{~kb}$ PCR product according to the previousl described spot transformation protocol (Stohl et al., 2001).

\subsubsection{Backcross or introduction of spontaneous deletion of $\triangle$ pilE into FA1090 parent or $\triangle O p a A-K$}

PCR was used to amplify $2.5 \mathrm{~kb}$ chromosomal region surrounding the spontaneous pilE deletion in FA1090. Table 2 contains primer sequences and Table 3 contains PCR conditions. This $2.5 \mathrm{~kb}$ PCR product was used to transform $\triangle \mathrm{OpaA}-\mathrm{K}$, and the FA1090 parent according to the spot transformation protocol.

\subsubsection{Screening and validation of pilE in frame and large deletions}

Phenotypically nonpiliated Gc were screened for the pilE in frame and large deletion by

PCR. Genomic DNA was used as a template and the primer combinations listed in Table 2 and the PCR conditions listed in Table 3. Potential in frame pilE clones in the $\triangle \mathrm{OpaBEGK}$ and $\triangle \mathrm{OpaA}-\mathrm{K}$ backgrounds were verified by Southern blotting. For Southern blotting, genomic DNA was restriction digested using Xho1 and ClaI (New England BioLabs) and probed with a pilE gene probe labeled with digoxigenin (Roche). 


\subsubsection{Bacterial survival assay}

Adherent PMNs were infected with exponential phase Gc and Gc survival was assessed by cell lysis and plating for viable CFU as previously described(Stohl et al., 2005).

\subsubsection{SDS-PAGE and Western Blotting}

7.2.6.1 Opa: SDS-PAGE and Western blotting for Opa expression was conducted as previously described(Criss et al., 2006). The 4B12 pan-Opa antibody hybridoma was obtained from Christof Hauck (University of Konstanz). Chemiluminescent blots were imaged with a ChemiDoc XRS_ system (Bio-Rad).

7.2.6.2 LOS: LOS was prepared from Gc as described by Davis and Goldberg (Davis et al., 2012), with the following modifications. Gc was collected after overnight growth on $\mathrm{GCB}$, and the protocol was stopped after the proteinase $\mathrm{K}$ treatment. Lysates were separated on 10 to $20 \%$ Tris-Tricine gradient gels (Bio-Rad) with a broad-range prestained protein marker (New England BioLabs). Gels were transferred to polyvinylidene difluoride (PVDF) and immunoblotted with the 2C7 monoclonal antibody, obtained from Michael Apicella (University of Iowa) followed by horseradish peroxidase-coupled goat anti-mouse antibody. Chemiluminescent blots were imaged with a ChemiDoc XRS_system (Bio-Rad).

7.2.6.3 PilC: SDS-PAGE and Western blotting for PilC expression was conducted as previously described (Criss et al., 2006). Rabbit anti-PilC antibody was used to detect PilC expression followed by a horseradish peroxidase-coupled goat anti-rabbit antibody. Chemiluminescent blots were imaged with a ChemiDoc XRS_system (Bio-Rad). 


\subsubsection{Immunofluorescence}

PMNs were infected for $1 \mathrm{~h}$ with Gc and fixed with 4\% PFA in PBS for 15 min. External and internal Gc were distinguished using a polyclonal rabbit anti-Gc antibody before and after PMN permeabilization as previously described in (Criss et al., 2009). Cells were then permeabilized using a 1:1 ratio of acetone and methanol. Post permeabilization, PMN primary granules were recognized using a monoclonal antibody against neutrophil elastase (AHN-10) (Millipore) followed by Alexa Fluor-coupled anti-mouse antibodies (Life Technologies). Coverslips were mounted using Flouromount G (Southern Biotech) with $2.5 \mathrm{mg} \mathrm{ml}^{-1}$ propyl gallate (Acros Organics). Images were acquired on a Nikon Eclipse E800 with Hamamatsu Orca-ER digital camera using Openlab software and processed in Adobe Photoshop CS5.

\subsection{Results}

Previous research indicates that piliated Gc have increased survival in the presence of PMNs compared to non-pilated Gc. We have demonstrated that phagosome maturity is one factor that determines Gc survival in PMNs (Johnson et al., 2013b). Our preliminary data indicate that nonpiliated Gc reside in a mature compartment while piliated Gc reside in an immature compartment (Figure 45), which may explain the observed survival defect of nonpiliated Gc in PMNs. In order to investigate the contribution of pili to Gc survival in PMNs, we generated an in frame deletion of pilE in the $\triangle \mathrm{OpaBEGK}$ and the $\triangle \mathrm{OpaA}-\mathrm{K}$ background using overlap extension PCR. CFU which displayed nonpiliated colony morphology after nonselective transformation were screened by PCR for deletion of pilE. PCR anaylsis showed transformants with a deletion of pilE in both the $\triangle \mathrm{OpaBEGK}$ and the $\triangle \mathrm{OpaA}-\mathrm{K}$ background (Figure 46A). The pilE 
deletion was also confirmed by Southern Blot (Figure 46B). To examine survival of $\triangle p i l E$ Gc after PMN challenge, adherent, IL-8 primed PMNs were infected with $\triangle$ OpaA$\mathrm{K}$ ApilE \#1 Gc, $\triangle \mathrm{OpaA}-\mathrm{K}$ ApilE \#2 Gc. Gc indicated as \#1 and \#2 refer to separate Gc transformants which contain a deletion of pilE. Survival of $\triangle \mathrm{OpaA}-\mathrm{K} \Delta p i l E \# 1 \mathrm{Gc}$ and $\Delta \mathrm{OpaA}-\mathrm{K}$ ApilE $\# 2 \mathrm{Gc}$ was compared to survival of $\Delta \mathrm{OpaA}-\mathrm{K} \mathrm{Gc}$ and the spontaneous FA1090 ApilE after PMN challenge. Similar to previous observations we observed that the background strain $\Delta \mathrm{OpaA}-\mathrm{K}$ Gc was initially killed after $30 \mathrm{~min}$ but recovered over the 90min time course. In agreement with previous reports, FA1090 spont $\Delta$ pilE Gc which contains the spontaneous deletion of pilE were readily killed by PMNs and survival did not recover. Surprisingly, $\Delta \mathrm{OpaA}-\mathrm{K} \Delta p i l E \# 1 \mathrm{Gc}$ and $\triangle \mathrm{OpaA}-\mathrm{K} \Delta$ pilE \#2 Gc survival resembled the survival curve of $\Delta$ OpaA-K Gc (Figure 46C); suggesting deletion of pilE does not affect Gc survival in PMNs.

Due to our results differing from previous reports, we generated an in frame deletion of pilE in the FA1090 background as described above. CFU which displayed nonpiliated colony morphology after nonselective transformation were screened by PCR for deletion of pilE. PCR results demonstrated successful deletion of pilE in the FA1090 background (Figure 47A). Survival of FA1090 ApilE after PMN challenge was assessed by infecting adherent, IL-8 primed PMNs with FA1090 ApilE \#1 or FA1090 ApilE \#2. Gc indicated as \#1 and \#2 refer to separate Gc transformants which contain a deletion of pilE. Survival was once again compared to survival of $\triangle$ OpaA-K Gc and FA1090 spont $\triangle$ pilE Gc after PMN challenge. After PMN challenge we observed no significant difference in survival of FA1090 ApilE \#1 and FA1090 $\triangle p i l E \# 2$ compared to $\Delta$ OpaA-K 
Gc (Figure 47B), supporting our previous observation that deletion of pilE does not alter Ge survival in PMNs.

The spontaneous 950bp deletion in FA1090 contains the pilE coding sequence and the surrounding region. Therefore, the survival defect observed for FA1090 spont $\Delta$ pilE after PMN challenge may be a result of the surrounding region that is deleted. In order to investigate this hypothesis we PCR amplified the chromosomal region surrounding the large pilE deletion in FA1090. This deletion will be referred to as large $\triangle$ pilE in order to differentiate it from the spontaneous pilE deletion in FA1090. This $2.5 \mathrm{~kb}$ PCR product was then used to transform FA1090 and $\triangle \mathrm{OpaA}-\mathrm{K}$ Gc in order to generate FA1090 large $\Delta p i l E$ and $\Delta$ OpaA-K large $\triangle p i l E$. These deletions were confirmed by PCR (Figure 48A and B). Survival of FA1090 large $4 p i l E \# 1$ and \#2 and $\triangle$ OpaA-K large $4 p i l E \# 1$ and \#2 was assessed after PMN challenge. Gc indicated as \#1 and \#2 refer to separate Gc transformants which contain a deletion of largeApilE. Survival of large $\Delta p i l E$ Gc mutants was compared to survival of $\Delta$ OpaA-K and FA1090 spont $\Delta p i l E$ Gc. We observed no significant difference in survival for FA1090 large $\Delta$ pilE or $\Delta$ OpaA$\mathrm{K}$ large $4 p i l E \mathrm{Gc}$ in PMNs compared to $\triangle \mathrm{OpaA}-\mathrm{K}$ Gc (Figure 48). Therefore, these data suggest that pilE is not required for Gc survival in the presence of PMNs.

\subsection{Discussion}

Gc piliation has been previously shown to provide a survival advantage in the presence of PMNs. However, early studies did not account for Opa protein expression which is known to influence Gc survival in PMNs, and more recent studies utilized Gc with a spontaneous deletion containing pilE and the surrounding region either of which could result in the observed survival defect in PMNs. Therefore, we generated an in 
frame deletion of pilE in our $\Delta \mathrm{OpaA}-\mathrm{K}$ in order to prevent confounding results due to Opa protein phase variation or deletion of the surrounding region. In contrast to previous reports, deletion of pilE did not significantly affect Gc survival in PMNs. In order to determine if differences in strain background or deletion of the region of pilE accounted for the previously observed survival defect in PMNs we generated three additional mutants: an in-frame deletion of pilE in the FA1090 parent background, a backcross of the spontaneous deletion into the variable FA1090 parent background, and introduction of the spontaneous deletion into the $\Delta \mathrm{OpaA}-\mathrm{K}$ background. In agreement with our initial results, we did not observe a significant survival defect for these mutants in PMNs. From our current results we conclude that pilE does not provide a Gc survival advantage in PMNs. However, the reasons for the discrepancy between previous results and our current findings remain unknown. As a result we did not continue to investigate the role of pili in Gc survival in PMNs.

We can envision two possibilities which may account for conflicting results on the role of pili in Gc survival in PMNs. First, our lab currently has unpublished data which indicates changes in lipooligosaccharide (LOS) affect Gc survival in PMNs. LOS is composed of three oligosaccharide chains attached to a lipid A core. The synthesis of LOS requires a series of phase variable glycosyl transferases which results in expression of different LOS isoforms (Danaher et al., 1995, Apicella et al., 1987). Therefore, Gc can express multiple LOS isoforms at any given time. Additionally, LOS can be modified by phosphoethanolamine (PEA) substitution on lipid A or the core oligosaccharide and by sialylation of the oligosaccharide (Plested et al., 1999, Mandrell et al., 1990). Addition of PEA or sialylation provides Gc resistance to normal human serum and cationic 
antimicrobial peptides (Shafer et al., 1986b, Lewis et al., 2009, Parsons et al., 1992). Gc sialylation also provides resistance to PMN killing and increases survival in the mouse model of infection (Wu et al., 2006a, Rest et al., 1992, Gill et al., 1996, Kim et al., 1992). The experiments discussed here did not account for differences in LOS which may affect Gc survival in PMNs. In order to begin addressing this hypothesis we examined the structure of LOS by conducting a western blot using the monoclonal antibody $2 \mathrm{C} 7$. Our unpublished data indicates that loss of the epitope for the $2 \mathrm{C} 7$ antibody results decreased survival in PMNs. Our results indicate that all the mutants used in this study express an isoform of LOS which is recognized by the 2C7 antibody, suggesting loss of the 2C7 epitope does not account for observed differences in survival (Figure 49A). Further investigation of LOS isoforms and LOS modifications in our pilE mutants may provide an explanation for the conflicting results regarding the role of piliation in Gc survival in PMNs.

Perhaps some of the most compelling data supporting a survival advantage for piliated Gc in PMNs is a mutation in pilQ results in a survival defect in PMNs. In the absence of pilQ, pili are not able to assemble and Gc are nonpiliated (Wolfgang et al., 2000, Drake et al., 1995). The pilE mutants in this study are still able to produce PilQ as well as minor pilin subunits. The second possibility to explain the differences in survival observed for FA1090 spont $\triangle p i l E$, a pilQ deletion mutant, and the pilE mutants used in this study is that minor pilin subunits or pilQ are present and sufficient for Gc survival in PMNs. In the absence of pilE, minor pilin subunits may be exposed on the Gc surface. One potential minor pilin subunit which could direct interactions with PMNs is PilC. PilC is phase variable and may account for the observed survival differences in PMNs 
between the FA1090 spont $\Delta$ pilE Gc used in previous studies and the Gc pilE mutants generated in this study. PilC is localized to the outer membrane and has been demonstrated to be important for adhesion to host cells (Soderholm et al., 2011, Kirchner et al., 2005b, Kirchner et al., 2005a, Rudel et al., 1995, Rahman et al., 1997). Therefore, exposure of PilC on the Gc surface may affect interactions with PMNs and result in differences in survival. We conducted a western blot to examine expression of PilC in the pilE mutants used in this study. Our results indicate all of the strains used in this study express PilC (Figure 49B). However, we did not examine if PilC was surface exposed in the Gc strains used in this study. Therefore, differences in PilC localization may still account for the observed differences in survival in PMNs. Gc pili are also composed of other minor pilin subunits and examination of these subunits may provide insight into the conflicting results presented in this study.

Two essential questions remain in the field regarding the role of pili in Gc survival in PMNs. First, are pili important for Gc binding to PMNs and does this mediate internalization? Early research suggests that pili are antiphagocytic (Swanson, 1974, Dilworth, 1975, Thomas, 1973). Gc survive better extracellularly in the context of PMNs (Criss et al., 2009, Johnson et al., 2011); therefore, preventing phagocytosis would provide a survival advantage in PMNs. There is also research indicating pili with PorB and LOS bind to complement receptor 3 on epithelial cells, providing a nonopsonic route of internalization(Edwards et al., 2005). This route of internalization has not been examined in PMNs and may provide an alternative route of entry. However, our preliminary results recapitulate previous data indicating that pili are not required for Gc internalization (Virji et al., 1986). In our immunofluorescence experiments investigating 
phagosome maturation we observed piliated and nonpiliated Gc were internalized to similar degree after 1 hour of infection $(65.0 \%$ and $69.0 \%)$. Further investigation is required to determine the route of piliated and nonpiliated Gc internalization. Second, how does Gc piliation affect PMN activation and Gc survival in PMNs? Pili have been shown to provide resistance to hydrogen peroxide and LL-37 in vitro and also to PMN mediated killing(Stohl et al., 2013). However, these studies did not determine if piliated Gc are surviving intracellularly or extracellularly in PMNs. Additionally, it would be important to evaluate the role pili in providing resistance to oxidative and non-oxidative components in the context of PMNs. There is substantial evidence indicating that the PMN oxidative burst is not required to mediate Gc killing, and Gc are killed primarily via non-oxidative components (Johnson et al., 2013b, Seib et al., 2005, Wu et al., 2009, Rest et al., 1982, Criss et al., 2008, Criss et al., 2009). Our lab has also demonstrated that Gc can avoid many of the PMN non-oxidative components by residing in immature phagosomes (Johnson et al., 2013b); however, the role of pili in this process is unknown. Understanding how Gc pili affect the route of Gc internalization, intracellular and extracellular survival in PMNs, and resistance to PMN killing would allow for generation of a more complete model to explain mechanisms of Gc survive in PMNs. 
7.5 Figures for Appendix C

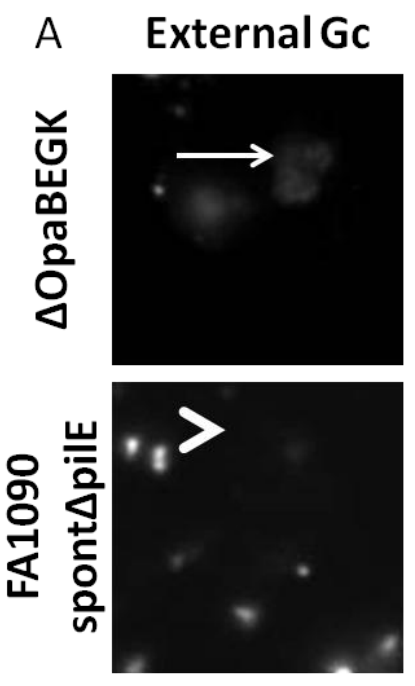

B

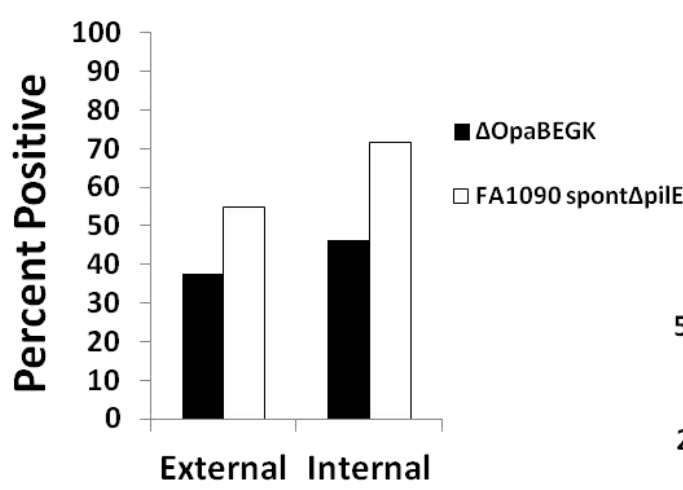

Internal Gc
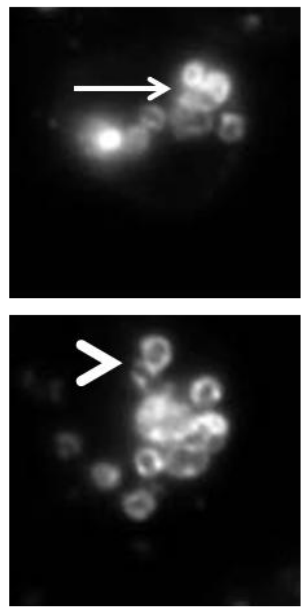

C
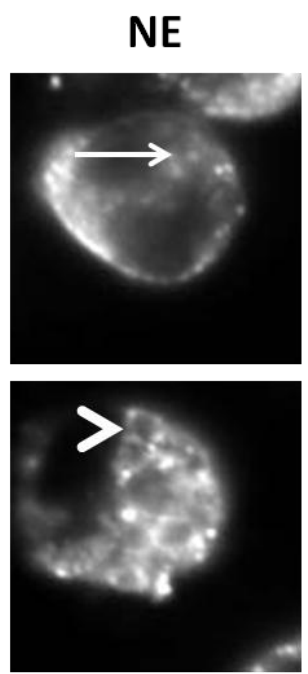

Merge
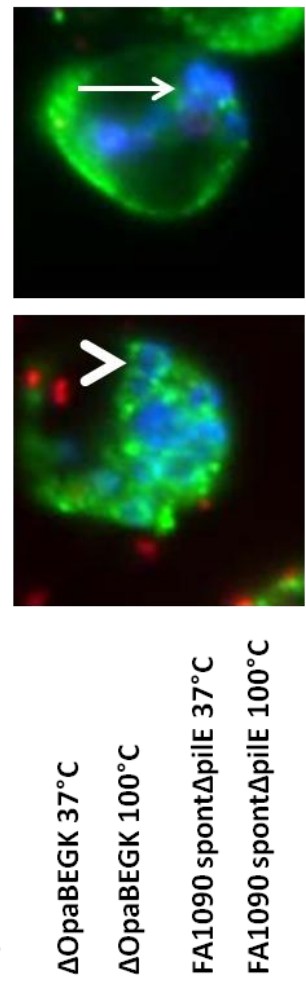

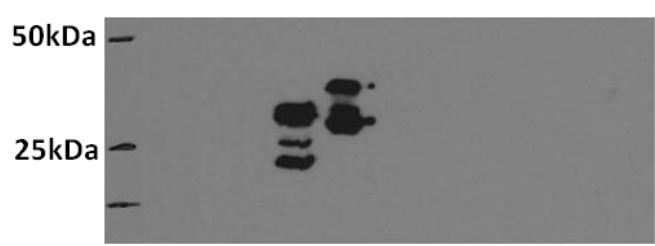

Figure 45: FA1090 spont $\Delta$ pilE are localized to mature phagosomes

A. Adherent, IL-8 treated, primary human PMNs were infected for $1 \mathrm{~h}$ with $\triangle$ OpaBEGK or FA1090 spont $\Delta$ pilE. Extracellular Gc appear red/blue, while intracellular Gc appear blue only. PMNs were stained with antibodies against the primary granule protein neutrophil elastase (NE), which appear green. Arrowheads indicate bacterial phagosomes positive for the NE and arrows indicate bacterial phagosomes negative for NE. The percent of Gc phagosomes positive for NE are reported in B. C. Lysates from $\triangle \mathrm{OpaA}-\mathrm{K}$, Gc expressing OpaB, $\triangle \mathrm{OpaBEGK}$, and FA1090 spont $\Delta$ pilE were incubated at $37^{\circ} \mathrm{C}$ or $100^{\circ} \mathrm{C}$ and then separated by SDSPAGE and immunoblotted with a pan-Opa antibody. Expression of one or more Opa proteins is presented only in the Gc expressing OpaB lysate as demonstrated by a heat-modifiable shift in reactivity with the Opa antibody. 


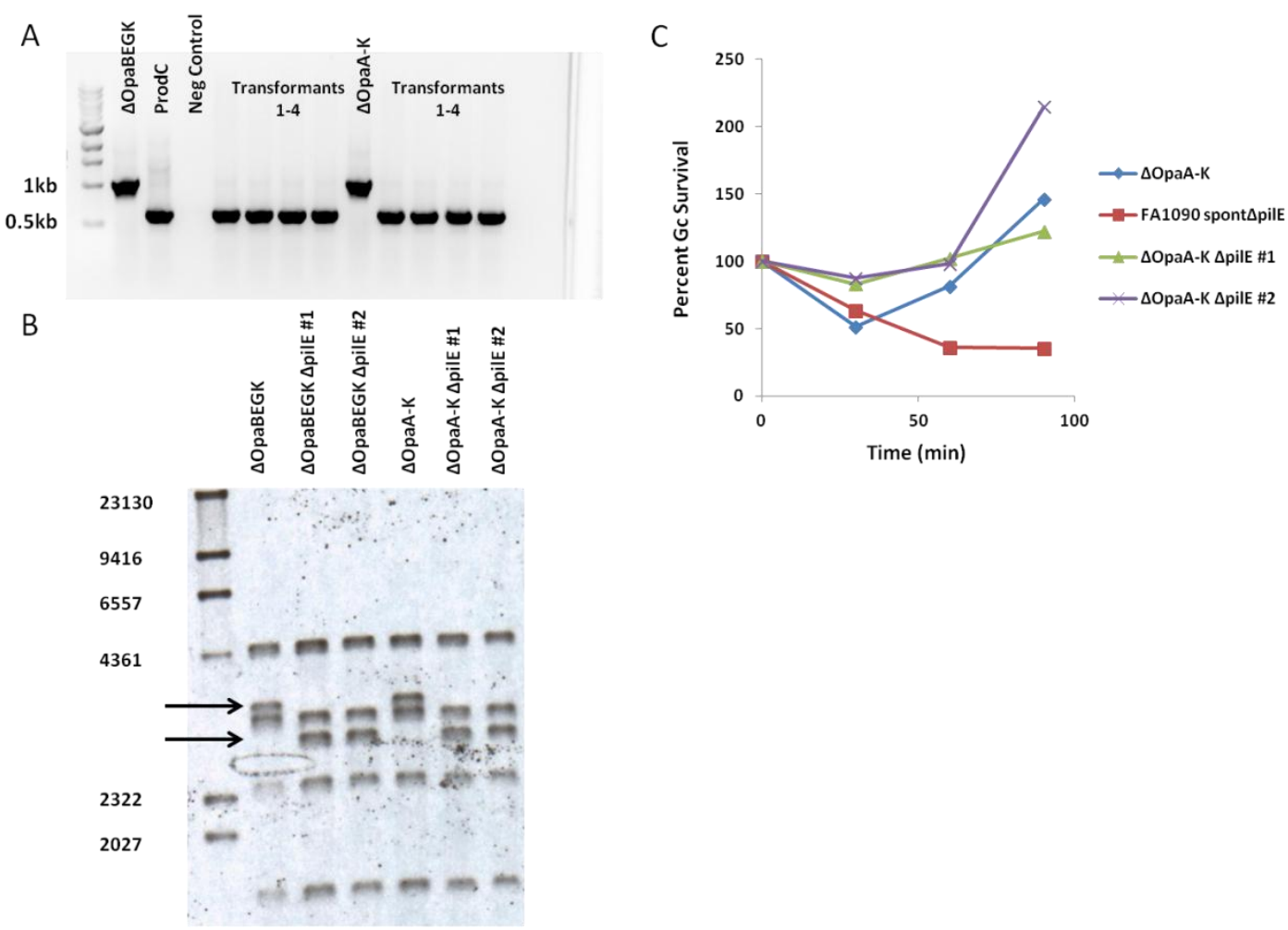

Figure 46: $\triangle$ OpaA-K $\Delta$ pilE Gc do not have significantly reduced survival in PMNs compared to $\triangle$ OpaA-K

A. Genomic DNA from $\triangle$ OpaBEGK, $\triangle \mathrm{OpaA}-\mathrm{K}$, and potential $\Delta$ pilE transformants in $\triangle \mathrm{OpaBEGK}$ and $\triangle \mathrm{OpaA}-\mathrm{K}$ background were used as template for $\mathrm{PCR}$, using the primer set PILRBS and OpaGscrdn1. Loss of pilE is indicated by the faster-migrating PCR product. B. Genomic DNA from $\triangle$ OpaBEGK, $\triangle$ OpaA-K, and potential $\Delta$ pilE transformants in $\triangle \mathrm{OpaBEGK}$ and $\triangle \mathrm{OpaA}-\mathrm{K}$ background was digested using the restriction enzymes Cla1 and Xho1. The digest was run on a DNA gel and probed using a PCR product generated using the primer set PilE prodB and OpaGscrdn1. Arrows indicate loss of pilE as shown by the faster-migrating digested DNA. C. Adherent, IL-8 primed PMNs were infected with $\Delta$ OpaA-K, FA1090 spont $\Delta$ pilE, or $\Delta$ OpaA-K $\Delta$ pilE. Bacterial survival over time was calculated as the CFU recovered from PMN lysates at each time point, divided by the CFU present at 0 min. 

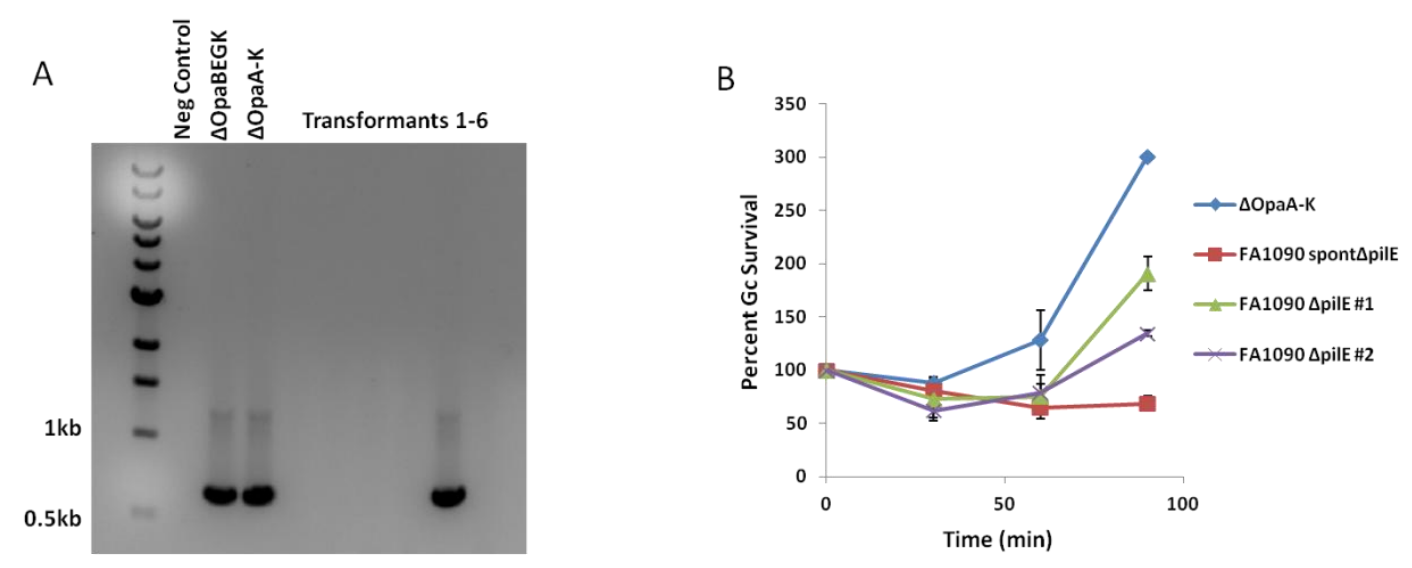

Figure 47: FA1090 $\Delta$ pilE Gc do not have a significantly reduced survival in PMNs compared to $\triangle$ OpaA-K
A. Genomic DNA from $\triangle \mathrm{OpaBEGK}, \triangle \mathrm{OpaA}-\mathrm{K}$, and potential $\Delta$ pilE transformants in FA1090 background were used as template for PCR, using the primer set PILRBS and SP3A. Loss of pilE is indicated by loss of the $0.65 \mathrm{~kb}$ band. B. Adherent, IL-8 primed PMNs were infected with $\Delta$ OpaA-K, FA1090 spont $\Delta$ pilE, or FA1090 $\Delta$ pilE. Bacterial survival over time was calculated as the CFU recovered from PMN lysates at each time point, divided by the $\mathrm{CFU}$ present at $0 \mathrm{~min}$. 

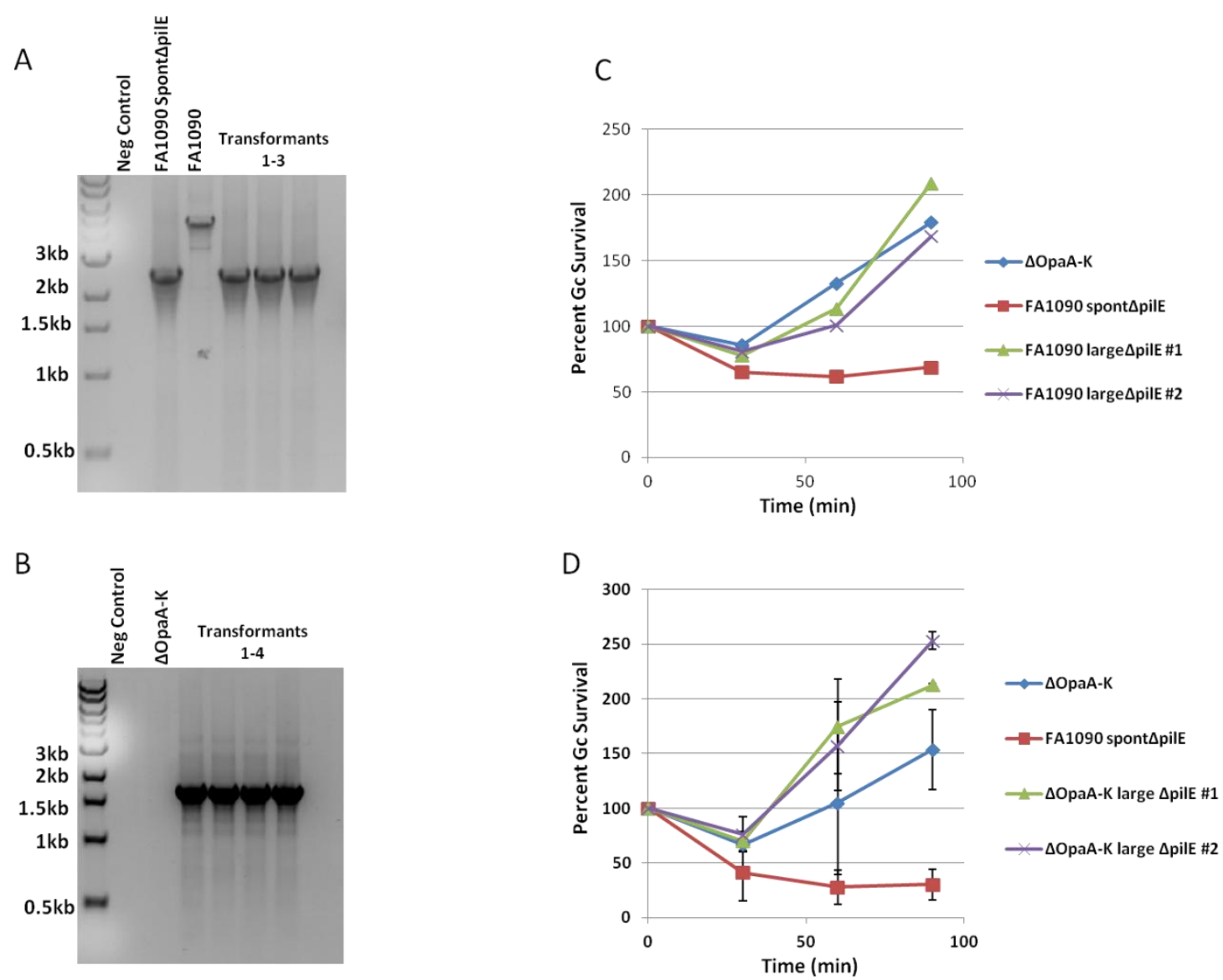

Figure 48: FA1090 large $\Delta$ pilE and $\Delta$ OpaA-K large $\Delta$ pilE Gc do not have signficiantly reduced survival in PMNs compared to $\triangle O p a A-K$

Genomic DNA from FA1090, FA1090 spont $\Delta$ pilE, $\Delta$ OpaA-K, potential large $\Delta$ pilE transformants in FA1090 background (A) and $\Delta$ OpaA-K (B) were used as template for PCR, using the primer set lpxC1 and OpaGscrdn1. A. Loss of pilE is indicated by the faster migrating PCR product. B. Loss of pilE is indicated by the presence of a $1.6 \mathrm{~kb}$ band. C-D. Adherent, IL-8 primed PMNs were infected with $\triangle$ OpaA-K, FA1090 spont $\Delta$ pilE, FA 1090 large $\Delta$ pilE, or $\Delta$ OpaA-K large $\Delta$ pilE. Bacterial survival over time was calculated as the CFU recovered from PMN lysates at each time point, divided by the CFU present at $0 \mathrm{~min}$. 
A

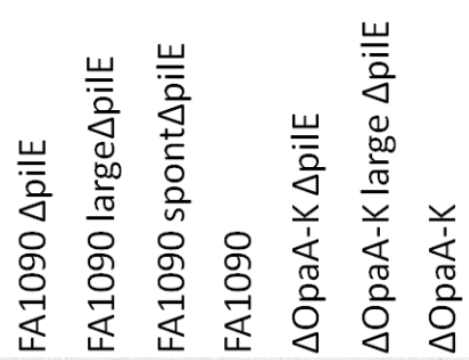

B

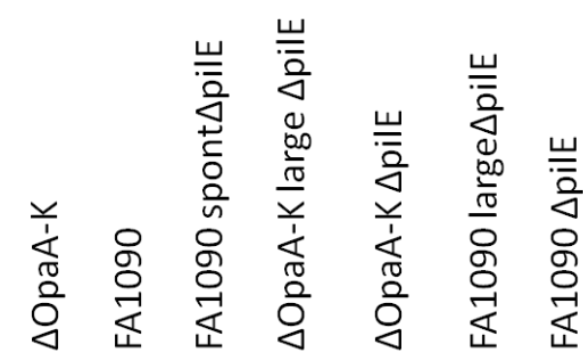

Figure 49: Gc strains used in this study express the 2C7 LOS epitope and PilC

A. Crude LOS was prepared from Gc strains FA1090 $\Delta$ pilE, FA1090 large $\Delta$ pilE, FA1090 spont $\Delta$ pilE, FA1090, $\Delta$ OpaA-K $\Delta$ pilE, $\Delta$ OpaA-K large $\Delta$ pilE, and $\Delta$ OpaA-K. The gel was transferred to PVDF for Western blotting with the $2 \mathrm{C} 7$ monoclonal antibody. B. Lysates from $\Delta$ OpaA-K, FA1090, FA1090 spont $\Delta$ pilE, $\Delta$ OpaA-K large $\Delta$ pilE, $\Delta$ OpaA-K $\Delta$ pilE, FA1090 large $\Delta$ pilE, and FA1090 $\Delta$ pilE were incubated at $100^{\circ} \mathrm{C}$ and then separated by SDSPAGE and immunoblotted with an anti-pilC antibody. 


\begin{tabular}{|l|l|}
\hline \multicolumn{1}{|c|}{ Primer Name } & \multicolumn{1}{|c|}{ Primer Sequence (5' '-3') } \\
\hline $\begin{array}{l}\text { PilE Forward } \\
\text { Mutagenesis }\end{array}$ & $\begin{array}{l}\text { GCCTAATTTGCCTTAGGTGGCAGACAGCTGTTATTGAAGGG } \\
\text { TATTCATAAAATTACTCC }\end{array}$ \\
\hline $\begin{array}{l}\text { PilE Reverse } \\
\text { Mutagenesis }\end{array}$ & $\begin{array}{l}\text { GGAGTAATTTTATGAATACCCTTCAATAACAGCTGTCTGCC } \\
\text { ACCTAAGGCAAATTAGGC }\end{array}$ \\
\hline OpaGscrdn1 & TTGGGCAACCGTTTTATCCG \\
\hline PilE prodB & GGCGTCGTCTTTGGCGA \\
\hline LPXC 1 & GGCTATCCCGATGAGTTTGT \\
\hline PILRBS & CCGGAACGGACGACCCCG \\
\hline SP3A & GCGGGCTGGATTCATTTTCGG \\
\hline OPAEJREV2 & \\
\hline USS1 &
\end{tabular}

Table 2: Primers used to generate pilE mutants 
194

\begin{tabular}{|l|l|l|l|}
\hline \multicolumn{1}{|c|}{ Purpose } & \multicolumn{1}{|c|}{ Primer combination } & \multicolumn{1}{|c|}{$\begin{array}{c}\text { Annealing } \\
\text { temp. }\end{array}$} & Polymerase \\
\hline pilE fragment A & $\begin{array}{l}\text { PilE ForMut and } \\
\text { OpaGscrndn1 }\end{array}$ & $56^{\circ} \mathrm{C}$ & $\begin{array}{l}\text { PFU II } \\
\text { Ultra }\end{array}$ \\
\hline pilE fragment B & PilE RevMut and PilEprodB & $56^{\circ} \mathrm{C}$ & $\begin{array}{l}\text { PFU II } \\
\text { Ultra }\end{array}$ \\
\hline $\begin{array}{l}\text { pilE OE-PCR } \\
\Delta \text { OpaA-K }\end{array}$ & PilEprodB and OpaGscrdn1 & $56^{\circ} \mathrm{C}$ & $\begin{array}{l}\text { PFU II } \\
\text { Ultra }\end{array}$ \\
\hline $\begin{array}{l}\text { Screen pilE deletion in } \\
\text { FA1090 }\end{array}$ & PILRBS and SP3A & $58^{\circ} \mathrm{C}$ & MangoTaq \\
\hline $\begin{array}{l}\text { Spontaneous pilE region } \\
\text { Screen pilE deletion in }\end{array}$ & PILRBS and OpaGscrdn1 & $58^{\circ} \mathrm{C}$ & MangoTaq \\
\hline $\begin{array}{l}\text { Screen large pilE } \\
\text { deletion in } \triangle \text { OpaA-K } \\
\text { deletion in FA1090 }\end{array}$ & LPXC1 and OpaGscrdn1 & $58^{\circ} \mathrm{C}$ & $\begin{array}{l}\text { PFU II } \\
\text { Ultra }\end{array}$ \\
\hline
\end{tabular}

Table 3: PCR conditions used to generate and screen pilE mutants. 


\section{Appendix D: PMN granule exocytosis in response to Gc infection}

\subsection{Introduction}

PMNs fuse cytoplasmic granules with the plasma membrane to upregulate receptors necessary for bacterial internalization and to release antimicrobial granule contents to the extracellular environment. PMNs exocytose secretory vesicles, tertiary granules, and secondary granules in order to upregulate receptors including cytokine receptors, TLRs, FcRs, and CD11b/CD18 for activation and recognition of microorganisms. Additionally, due to secondary and primary granule exocytosis, bacteria would be exposed to ROS, antimicrobial peptides, and proteases which could kill bacteria at the surface (Faurschou et al., 2003). Due to the receptors and antimicrobial components contained in granules, altered PMN granule exocytosis could result in changes in bacterial uptake and killing.

Previous research indicates that Opa+ Gc have a survival defect in PMNs compared to Opa- Gc (Rest et al., 1982, Virji et al., 1986, Criss et al., 2009, Ball et al., 2013). We have demonstrated that the Opa+ Gc survival defect is primarily intracellular; however, we did observe a decrease in extracellular survival (Chapter 3). Previous data indicates that Opa+ Gc engagement of CEACAM receptors on PMNs stimulates increased granule exocytosis compared to Opa- Gc (Sarantis et al., 2007). In order to determine if infection of adherent, IL-8 primed PMNs with Opa+ Gc results in increased PMN granule exocytosis, we compared the surface expression of PMN granules proteins between suspension PMNs, uninfected adherent, IL-8 primed PMNs, and Opa+ and OpaGc infected adherent PMNs using flow cytometry. Our preliminary data indicates that 
PMN priming and adherence stimulates primary and secondary granule exocytosis. However, we did not observe a significant difference in exocytosis in response to infection with Opa+ or Opa- Gc. Our results suggest that infection with Opa+ Gc does not stimulate increased PMN granule exocytosis in adherent, IL-8 primed PMNs compared to Opa- Gc.

\subsection{Materials and Methods}

\subsubsection{Bacterial strains and growth conditions}

The Gc strains used in this study are in the FA1090 background which constitutively expresses the pilin variant 1-81-S2 due to mutation of the guanine quartet sequence upstream of pilE (Cahoon et al., 2009). The majority of experiments were conducted using Opaless and $\mathrm{OpaD}_{\mathrm{nv}}($ Ball et al., 2013), referred to here as "Opa-" and "Opa+" Gc respectively. The strain defined as Opa- has in-frame deletions of the genes encoding the 11 opacity-associated (Opa) proteins(Ball et al., 2013). The strain defined as OpaD+ was generated in the Opa- background and is locked for expression of OpaD due to replacement of the CTCTT repeat-rich region of FA1090 opaD with a sequence which is unable to undergo phase variation(Ball et al., 2013). Piliated Gc was routinely grown on Gonococcal Medium Base (Difco) plus Kellogg's supplements(Kellogg et al., 1963) for $20 \mathrm{~h}$ at $37^{\circ} \mathrm{C}$ in $5 \% \mathrm{CO}_{2}$. Gc was grown to exponential phase via successive rounds of bacterial growth in rich liquid medium (GCBL).

\subsubsection{Suspension PMNs}

$10^{6}$ PMNs for each staining condition were fixed with $1 \%$ PFA for $10 \mathrm{~min}$ on ice and processed for flow cytometry.

\subsubsection{Adherent fMLP stimulated PMNs}


$10^{6}$ PMNs were primed with $10 \mathrm{nM}$ IL-8 (R\&D systems) and adhered to $25 \mathrm{~mm}$ tissue culture plastic coverslips for $30 \mathrm{~min}$ at $37^{\circ} \mathrm{C}$. Cells were treated with $5 \mu \mathrm{g} / \mathrm{ml}$ cytochalsin B for $10 \mathrm{~min}$ at $37^{\circ} \mathrm{C}$. Cells were then treated with $1 \mu \mathrm{M}$ Formyl-Methionyl-LeucylPhenylalanine (fMLP) for $10 \mathrm{~min}$ at $37^{\circ} \mathrm{C}$. Cells were fixed with $1 \%$ PFA for $10 \mathrm{~min}$ on ice. Cells were scraped and collected in a $15 \mathrm{ml}$ conical tube. Cells were spun down at $600 \mathrm{Xg}$ for $4 \mathrm{~min}$ at $4^{\circ} \mathrm{C}$ and processed for flow cytometry.

\subsubsection{Gc infection of PMNs}

$10^{6}$ PMNs were primed with $10 \mathrm{nM}$ IL-8 (R\&D systems) and adhered to $25 \mathrm{~mm}$ tissue culture plastic coverslips for $30 \mathrm{~min}$ at $37^{\circ} \mathrm{C}$. PMNs were incubated for 5 min on ice prior to infection with Gc. PMNs were infected with Gc at a multiplicity of infection of 1-5 bacterial colony forming units (CFU) per PMN as described in (Criss et al., 2009). PMNs were infected for $30 \mathrm{~min}$ at $37^{\circ} \mathrm{C}$ with $\mathrm{Gc}$ followed by fixation with $1 \%$ PFA for $10 \mathrm{~min}$ on ice. Cells were scraped and collected in a $15 \mathrm{ml}$ conical tube. Cells were spun down at $600 \mathrm{Xg}$ for $4 \mathrm{~min}$ at $4^{\circ} \mathrm{C}$ and processed for flow cytometry.

\subsubsection{Staining PMNs and Flow cytometry}

Cells were blocked in 10\% Normal Goat Serum (Gibco) in 1XPBS. PMN primary granules were recognized using a FITC coupled anti-CD63 antibody (Ancell) and PMN secondary granules were recognized using a FITC coupled anti-CD66b antibody (PeliCluster). A FITC Mouse IgG1 antibody (BD Pharmingen) was used as an isotype control. Cells were run on a Becton Dickinson/Cytek FACSCalibur Benchtop Analyzers and analyzed with FlowJo software (Tree Star Inc). 


\subsection{Results}

We hypothesized that Opa+ Gc stimulate increased PMN granule exocytosis compared to Opa- Gc resulting in the observed survival defect. In order to evaluate PMN granule exocytosis in response to infection with Opa+ and Opa- Gc, adherent, IL-8 primed PMNs were infected with either Opa+ or Opa- Gc. PMN granule exocytosis was evaluated by examining surface expression of the primary granule protein, CD63 and the secondary granule protein, CD66b via flow cytometry. We observed that adherent, IL-8 primed PMNs have increased primary and secondary granule exocytosis compared to suspension PMNs. There was no significant difference in primary or secondary granule exocytosis in response to infection with Opa+ and Opa- Gc. In order to stimulate maximal granule exocytosis, adherent, IL-8 primed PMNs were treated with Cytochalasin B followed by stimulation with Formyl-Methionyl-Leucyl-Phenylalanine (fMLP). We observed that stimulation via fMLP was sufficient to increase PMN granule exocytosis above levels observed for uninfected and Gc infected PMNs (Figure 50). From these data, we conclude that Opa+ and Opa- Gc infection of adherent, IL-8 primed PMNs stimulates similar amounts of primary and secondary granule exocytosis.

\subsection{Discussion}

Our previous results indicate that Opa+ Gc have reduced external survival compared to Opa- Gc. We observed that Opa+ and Opa- Gc infection of adherent, IL-8 primed PMNs stimulated similar amounts of primary and secondary granule exocytosis. Therefore, decreased Opa+ Gc survival in PMNs is not due to differences in granule exocytosis. 
There are two remaining hypotheses to explain the decrease in external survival of Opa+ Gc compared to Opa- Gc in PMNs. First, Opa+ Gc stimulate increased production of neutrophil extracellular traps (NETs). NETs are composed of decondensed chromatin and PMN granule proteins which are released into the extracellular environment to form web like structures which trap and kill microorganisms(Papayannopoulos et al., 2009). Increased production of NETs in response to infection with Opa+ Gc may result in increased exposure to antimicrobial components. In order to test this hypothesis, fluorescence microscopy could be used to evaluate the amount of NET production in response to infection with Opa+ and Opa- Gc.

The second possibility is that Opa+ Gc have decreased external survival due to increased sensitivity to PMN antimicrobial components compared to Opa- Gc. In the external environment Gc would be exposed to PMN granule components and NETs. We have previously demonstrated that Opa+ do not have increased sensitivity to the antigonococcal components LL-37 and cathepsin G (Chapter 3). However, these are only two of the many components found in PMN granules. We have demonstrated that PMN protease activity is essential for intracellular killing of Gc (Johnson et al., 2013b). Therefore, increased sensitivity of Opa+ Gc to other proteases found in PMN granules, such as neutrophil elastase and proteinase 3, may account for the observed external survival decrease. An alternative is that Opa+ Gc are more sensitive to antimicrobial histones found in NETs (Brinkmann et al., 2004). In order to determine if Opa+ Gc have increased sensitivity to PMN antimicrobial components compared to Opa- Gc, future experiments could compare survival of Opa+ and Opa- Gc in presence of neutrophil elastase, proteinase 3 , or histones. 
The data presented here provide evidence which indicates reduced external survival of Opa+ Gc is not due to increased PMN granule exocytosis. Future experiments to address production of NETs in response to infection with Opa+ Gc and the sensitivity of Opa+ Gc to PMN extracellular killing may provide insight into the mechanism of PMN extracellular killing of Opa+ Gc.

The flow cytometry experiment designed to evaluate PMN granule exocytosis in adherent PMNs will provide a technique to address other critical questions. There is substantial evidence in the field indicating that Opa proteins are required for PMN internalization of Gc (Belland et al., 1992, Virji et al., 1986, Fischer et al., 1988, King et al., 1978b, Gray-Owen et al., 1997a). In contrast, our lab has demonstrated that Opa- Gc are still internalized by PMNs (Johnson et al., 2013b, Criss et al., 2009, Ball et al., 2013). One possible explanation for the contrasting reports on the requirement for Opa proteins in Gc internalization is differences in PMN activation state influence Gc internalization. Previous research investigating the role of Opa proteins in Gc internalization used suspension PMNs, while our experiments use adherent, IL-8 primed PMNs. The flow cytometry technique discussed here could be used to evaluate differences in PMN activation for suspension and adherent, IL-8 primed PMNs. Our preliminary results indicate that IL-8 priming and PMN adherence stimulate an increase in secondary and primary granule release compared to suspension PMNs (Figure 50). Evaluating the amount of granule exocytosis for all PMN granule classes would reveal the abundance of receptors present on the PMN surface and may provide insight into the mechanisms of PMN internalization of Opa- Gc, which is currently unknown. Further investigation of PMN activation state and how Opa- Gc are internalized would assist in development of a 
complete model to explain Gc residence in immature phagosomes which allows for intracellular survival in PMNs. 


\subsection{Figures for Appendix D}

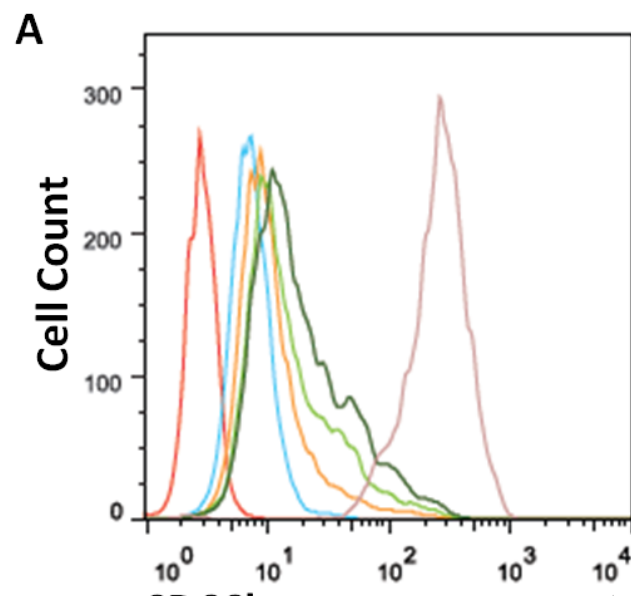

CD66b

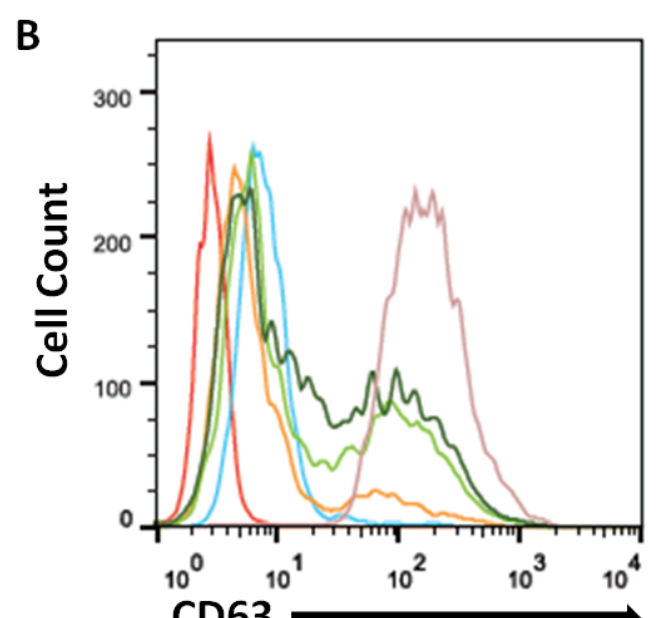

CD63

FITC Isotype Suspension Uninfected

Suspension Uninfected

Adherent Uninfected

Adherent Opa- infected

Adherent fMLP stimulated

Figure 50: PMN adherence stimulates secondary and primary granule exocytosis

A-B. Secondary and primary granule exocytosis was compared for suspension PMNs, Adherent PMNs; Adherent PMNs treated with $5 \mu \mathrm{g} / \mathrm{ml}$ cytochalsin B followed by stimulation with fMLP, Adherent PMNs infected with Opa- Gc, and Adherent PMNs infected with Opa+ Gc. A. Representative histogram for levels of CD66b staining which was used to mark secondary granules. B. Representative histogram for levels of CD63 staining which was used to mark primary granules. 
Abdel-Latif, D., Steward, M. and Lacy, P. (2005). Neutrophil primary granule release and maximal superoxide generation depend on Rac2 in a common signalling pathway. Can J Physiol Pharmacol 83, 69-75.

Abdel-Latif, D., Steward, M., Macdonald, D.L., Francis, G.A., Dinauer, M.C. and Lacy, P. (2004). Rac2 is critical for neutrophil primary granule exocytosis. Blood 104, 832-839.

Achard, M.E., Hamilton, A.J., Dankowski, T., Heras, B., Schembri, M.S., Edwards, J.L., et al. (2009). A periplasmic thioredoxin-like protein plays a role in defense against oxidative stress in Neisseria gonorrhoeae. Infect Immun 77, 4934-4939.

Allen, L.A. (2007). Immunofluorescence and confocal microscopy of neutrophils. Methods Mol Biol 412, 273-287.

Allen, L.A., Schlesinger, L.S. and Kang, B. (2000). Virulent strains of Helicobacter pylori demonstrate delayed phagocytosis and stimulate homotypic phagosome fusion in macrophages. $J$ Exp Med 191, 115-128.

Amer, A.O. and Swanson, M.S. (2002). A phagosome of one's own: a microbial guide to life in the macrophage. Curr Opin Microbiol 5, 56-61.

Apicella, M.A., Ketterer, M., Lee, F.K., Zhou, D., Rice, P.A. and Blake, M.S. (1996). The pathogenesis of gonococcal urethritis in men: confocal and immunoelectron microscopic analysis of urethral exudates from men infected with Neisseria gonorrhoeae. J Infect Dis 173, 636-646.

Apicella, M.A., Shero, M., Jarvis, G.A., Griffiss, J.M., Mandrell, R.E. and Schneider, H. (1987). Phenotypic variation in epitope expression of the Neisseria gonorrhoeae lipooligosaccharide. Infect Immun 55, 1755-1761.

Arcaro, A. and Wymann, M.P. (1993). Wortmannin is a potent phosphatidylinositol 3kinase inhibitor: the role of phosphatidylinositol 3,4,5-trisphosphate in neutrophil responses. Biochem J 296 ( Pt 2), 297-301.

Archibald, F.S. and Duong, M.N. (1984). Manganese acquisition by Lactobacillus plantarum. J Bacteriol 158, 1-8.

Archibald, F.S. and Duong, M.N. (1986). Superoxide dismutase and oxygen toxicity defenses in the genus Neisseria. Infect Immun 51, 631-641.

Astarie-Dequeker, C., Nigou, J., Puzo, G. and Maridonneau-Parini, I. (2000). Lipoarabinomannans activate the protein tyrosine kinase Hck in human neutrophils. Infect Immun 68, 4827-4830.

Ball, L.M. and Criss, A.K. (2013). Constitutively Opa-Expressing and Opa-Deficient Neisseria gonorrhoeae Strains Differentially Stimulate and Survive Exposure to Human Neutrophils. J Bacteriol 195, 2982-2990.

Balthazar, J.T., Gusa, A., Martin, L.E., Choudhury, B., Carlson, R. and Shafer, W.M. (2011). Lipooligosaccharide Structure is an Important Determinant in the Resistance of Neisseria Gonorrhoeae to Antimicrobial Agents of Innate Host Defense. Front Microbiol 2, 30.

Bangalore, N., Travis, J., Onunka, V.C., Pohl, J. and Shafer, W.M. (1990). Identification of the primary antimicrobial domains in human neutrophil cathepsin G. J Biol Chem 265, 13584-13588. 
Bauer, F.J., Rudel, T., Stein, M. and Meyer, T.F. (1999). Mutagenesis of the Neisseria gonorrhoeae porin reduces invasion in epithelial cells and enhances phagocyte responsiveness. Mol Microbiol 31, 903-913.

Belland, R.J., Chen, T., Swanson, J. and Fischer, S.H. (1992). Human neutrophil response to recombinant neisserial Opa proteins. Mol Microbiol 6, 1729-1737.

Belland, R.J., Nelson, D. E., Virok, D., Crane, D. D., Hogan, D., Sturdevant, D., Beatty, W. L., and Caldwell, H.D. (2003). Transcriptome analysis of chlamydial growth during IFN-y-mediated persistence and reactivation. Proceedings of the National Academy of Sciences of the United States of America 100, 15971-15976.

Bhat, K.S., Gibbs, C.P., Barrera, O., Morrison, S.G., Jahnig, F., Stern, A., et al. (1991). The opacity proteins of Neisseria gonorrhoeae strain MS11 are encoded by a family of 11 complete genes. Mol Microbiol 5, 1889-1901.

Bjerknes, R., Guttormsen, H.K., Solberg, C.O. and Wetzler, L.M. (1995). Neisserial porins inhibit human neutrophil actin polymerization, degranulation, opsonin receptor expression, and phagocytosis but prime the neutrophils to increase their oxidative burst. Infect Immun 63, 160-167.

Blake, M.S., Gotschlich, E.C. and Swanson, J. (1981). Effects of proteolytic enzymes on the outer membrane proteins of Neisseria gonorrhoeae. Infect Immun 33, 212-222.

Blake, M.S. and Swanson, J. (1978). Studies on gonococcus infection. XVI. Purification of Neisseria gonorrhoeae immunoglobulin A1 protease. Infect Immun 22, 350358.

Booth, J.W., Telio, D., Liao, E.H., McCaw, S.E., Matsuo, T., Grinstein, S. and GrayOwen, S.D. (2003). Phosphatidylinositol 3-kinases in carcinoembryonic antigenrelated cellular adhesion molecule-mediated internalization of Neisseria gonorrhoeae. J Biol Chem 278, 14037-14045.

Borregaard, N. (2010). Neutrophils, from marrow to microbes. Immunity 33, 657-670.

Borregaard, N., Sorensen, O.E. and Theilgaard-Monch, K. (2007). Neutrophil granules: a library of innate immunity proteins. Trends Immunol 28, 340-345.

Bos, M.P., Kuroki, M., Krop-Watorek, A., Hogan, D. and Belland, R.J. (1998). CD66 receptor specificity exhibited by neisserial Opa variants is controlled by protein determinants in CD66 N-domains. Proc Natl Acad Sci U S A 95, 9584-9589.

Boslego, J.W., Tramont, E.C., Chung, R.C., McChesney, D.G., Ciak, J., Sadoff, J.C., et al. (1991). Efficacy trial of a parenteral gonococcal pilus vaccine in men. Vaccine 9, 154-162.

Botha, M., Botes, M., Loos, B., Smith, C. and Dicks, L.M. (2012). Lactobacillus equigenerosi strain Le1 invades equine epithelial cells. Appl Environ Microbiol 78, 4248-4255.

Bozzola, J.J. (2007). Conventional specimen preparation techniques for transmission electron microscopy of cultured cells. Methods Mol Biol 369, 1-18.

Brinkmann, V., Reichard, U., Goosmann, C., Fauler, B., Uhlemann, Y., Weiss, D.S., et al. (2004). Neutrophil extracellular traps kill bacteria. Science 303, 1532-1535.

Britigan, B.E., Klapper, D., Svendsen, T. and Cohen, M.S. (1988). Phagocyte-derived lactate stimulates oxygen consumption by Neisseria gonorrhoeae. An unrecognized aspect of the oxygen metabolism of phagocytosis. J Clin Invest 81, 318-324. 
Brogden, K.A. (2005). Antimicrobial peptides: pore formers or metabolic inhibitors in bacteria? Nat Rev Microbiol 3, 238-250.

Brooks, G.F., Israel, K.S. and Petersen, B.H. (1976). Bactericidal and opsonic activity against Neisseria gonorrhoeae in sera from patients with disseminated gonococcal infection. J Infect Dis 134, 450-462.

Brumell, J.H., Volchuk, A., Sengelov, H., Borregaard, N., Cieutat, A.M., Bainton, D.F., et al. (1995). Subcellular distribution of docking/fusion proteins in neutrophils, secretory cells with multiple exocytic compartments. J Immunol 155, 5750-5759.

Brzezinska, A.A., Johnson, J.L., Munafo, D.B., Ellis, B.A. and Catz, S.D. (2009).

Signalling mechanisms for Toll-like receptor-activated neutrophil exocytosis: key roles for interleukin-1-receptor-associated kinase-4 and phosphatidylinositol 3kinase but not Toll/IL-1 receptor (TIR) domain-containing adaptor inducing IFNbeta (TRIF). Immunology 127, 386-397.

Buntru, A., Roth, A., Nyffenegger-Jann, N.J. and Hauck, C.R. (2012). HemITAM signaling by CEACAM3, a human granulocyte receptor recognizing bacterial pathogens. Arch Biochem Biophys 524, 77-83.

Cahoon, L.A. and Seifert, H.S. (2009). An alternative DNA structure is necessary for pilin antigenic variation in Neisseria gonorrhoeae. Science 325, 764-767.

Cahoon, L.A. and Seifert, H.S. (2011). Focusing homologous recombination: pilin antigenic variation in the pathogenic Neisseria. Mol Microbiol 81, 1136-1143.

Caron, E. and Hall, A. (1998). Identification of two distinct mechanisms of phagocytosis controlled by different Rho GTPases. Science 282, 1717-1721.

Casey, S.G., Shafer, W.M. and Spitznagel, J.K. (1985). Anaerobiosis increases resistance of Neisseria gonorrhoeae to O2-independent antimicrobial proteins from human polymorphonuclear granulocytes. Infect Immun 47, 401-407.

Casey, S.G., Shafer, W.M. and Spitznagel, J.K. (1986). Neisseria gonorrhoeae survive intraleukocytic oxygen-independent antimicrobial capacities of anaerobic and aerobic granulocytes in the presence of pyocin lethal for extracellular gonococci. Infect Immun 52, 384-389.

Casey, S.G., Veale, D.R. and Smith, H. (1979). Demonstration of intracellular growth of gonococci in human phagocytes using spectinomycin to kill extracellular organisms. J Gen Microbiol 113, 395-398.

Casey, S.G., Veale, D.R. and Smith, H. (1980). Intracellular survival of Neisseria gonorrhoeae in human urethral exudate. FEMS Microbiol Lett 8, 97-100.

Chen, A. and Seifert, H.S. (2011). Neisseria gonorrhoeae-mediated inhibition of apoptotic signalling in polymorphonuclear leukocytes. Infect Immun.

Chen, T. and Gotschlich, E.C. (1996). CGM1a antigen of neutrophils, a receptor of gonococcal opacity proteins. Proc Natl Acad Sci U S A 93, 14851-14856.

Chen, T., Grunert, F., Medina-Marino, A. and Gotschlich, E.C. (1997). Several carcinoembryonic antigens (CD66) serve as receptors for gonococcal opacity proteins. J Exp Med 185, 1557-1564.

Chow, O.A., von Kockritz-Blickwede, M., Bright, A.T., Hensler, M.E., Zinkernagel, A.S., Cogen, A.L., et al. (2010). Statins enhance formation of phagocyte extracellular traps. Cell Host Microbe 8, 445-454. 
Cohen, M.S. and Cannon, J.G. (1999). Human experimentation with Neisseria gonorrhoeae: progress and goals. J Infect Dis 179 Suppl 2, S375-379.

Cole, J.G., Fulcher, N.B. and Jerse, A.E. (2010). Opacity proteins increase Neisseria gonorrhoeae fitness in the female genital tract due to a factor under ovarian control. Infect Immun 78, 1629-1641.

Collins, S.J., Gallo, R.C. and Gallagher, R.E. (1977). Continuous growth and differentiation of human myeloid leukaemic cells in suspension culture. Nature 270, 347-349.

Connell, T.D., Black, W.J., Kawula, T.H., Barritt, D.S., Dempsey, J.A., Kverneland, K., Jr., et al. (1988). Recombination among protein II genes of Neisseria gonorrhoeae generates new coding sequences and increases structural variability in the protein II family. Mol Microbiol 2, 227-236.

Connell, T.D., Shaffer, D. and Cannon, J.G. (1990). Characterization of the repertoire of hypervariable regions in the Protein II (opa) gene family of Neisseria gonorrhoeae. Mol Microbiol 4, 439-449.

Cougoule, C., Constant, P., Etienne, G., Daffe, M. and Maridonneau-Parini, I. (2002). Lack of fusion of azurophil granules with phagosomes during phagocytosis of Mycobacterium smegmatis by human neutrophils is not actively controlled by the bacterium. Infect Immun 70, 1591-1598.

Criss, A.K., Katz, B.Z. and Seifert, H.S. (2009). Resistance of Neisseria gonorrhoeae to non-oxidative killing by adherent human polymorphonuclear leucocytes. Cell Microbiol 11, 1074-1087.

Criss, A.K. and Seifert, H.S. (2006). Gonococci exit apically and basally from polarized epithelial cells and exhibit dynamic changes in type IV pili. Cell Microbiol 8, 1430-1443.

Criss, A.K. and Seifert, H.S. (2008). Neisseria gonorrhoeae suppresses the oxidative burst of human polymorphonuclear leukocytes. Cell Microbiol 10, 2257-2270.

Criss, A.K. and Seifert, H.S. (2012). A bacterial siren song: intimate interactions between Neisseria and neutrophils. Nat Rev Microbiol 10, 178-190.

Danaher, R.J., Levin, J.C., Arking, D., Burch, C.L., Sandlin, R. and Stein, D.C. (1995). Genetic basis of Neisseria gonorrhoeae lipooligosaccharide antigenic variation. $J$ Bacteriol 177, 7275-7279.

Davidsen, T., Bjoras, M., Seeberg, E.C. and Tonjum, T. (2005). Antimutator role of DNA glycosylase MutY in pathogenic Neisseria species. J Bacteriol 187, 2801-2809.

Davis, M.R., Jr. and Goldberg, J.B. (2012). Purification and visualization of lipopolysaccharide from Gram-negative bacteria by hot aqueous-phenol extraction. $J$ Vis Exp.

de Jonge, M.I., Hamstra, H.J., van Alphen, L., Dankert, J. and van der Ley, P. (2003). Mapping the binding domains on meningococcal Opa proteins for CEACAM1 and CEA receptors. Mol Microbiol 50, 1005-1015.

Dempsey, J.A., Litaker, W., Madhure, A., Snodgrass, T.L. and Cannon, J.G. (1991). Physical map of the chromosome of Neisseria gonorrhoeae FA1090 with locations of genetic markers, including opa and pil genes. J Bacteriol 173, 54765486. 
Densen, P. and Mandell, G.L. (1978). Gonococcal interactions with polymorphonuclear neutrophils: importance of the phagosome for bactericidal activity. J Clin Invest 62, 1161-1171.

Desjardins, M. and Descoteaux, A. (1997). Inhibition of phagolysosomal biogenesis by the Leishmania lipophosphoglycan. J Exp Med 185, 2061-2068.

Dilworth, J., Hendley, J. O., and Mandell, G. L. (1975). Attachment and Ingestion of Gonococci by Human Neutrophils. Infection And Immunity 11, 512-516.

Dilworth, J.A., Hendley, J.O. and Mandell, G.L. (1975). Attachment and ingestion of gonococci human neutrophils. Infect Immun 11, 512-516.

Dorward, D.W. (2008). Ultrastructural analysis of bacteria-host cell interactions. Methods Mol Biol 431, 173-187.

Drake, S.L. and Koomey, M. (1995). The product of the pilQ gene is essential for the biogenesis of type IV pili in Neisseria gonorrhoeae. Mol Microbiol 18, 975-986.

Draper, D.L., James, J.F., Brooks, G.F. and Sweet, R.L. (1980). Comparison of virulence markers of peritoneal and fallopian tube isolates with endocervical Neisseria gonorrhoeae isolates from women with acute salpingitis. Infect Immun 27, 882888.

Edwards, A.M. and Massey, R.C. (2011). Invasion of human cells by a bacterial pathogen. $J$ Vis Exp.

Edwards, J.L. and Apicella, M.A. (2002a). The role of lipooligosaccharide in Neisseria gonorrhoeae pathogenesis of cervical epithelia: lipid A serves as a $\mathrm{C} 3$ acceptor molecule. Cell Microbiol 4, 585-598.

Edwards, J.L. and Apicella, M.A. (2004). The molecular mechanisms used by Neisseria gonorrhoeae to initiate infection differ between men and women. Clin Microbiol Rev 17, 965-981, table of contents.

Edwards, J.L. and Apicella, M.A. (2005). I-domain-containing integrins serve as pilus receptors for Neisseria gonorrhoeae adherence to human epithelial cells. Cell Microbiol 7, 1197-1211.

Edwards, J.L., Brown, E.J., Ault, K.A. and Apicella, M.A. (2001). The role of complement receptor 3 (CR3) in Neisseria gonorrhoeae infection of human cervical epithelia. Cell Microbiol 3, 611-622.

Edwards, J.L., Brown, E.J., Uk-Nham, S., Cannon, J.G., Blake, M.S. and Apicella, M.A. (2002b). A co-operative interaction between Neisseria gonorrhoeae and complement receptor 3 mediates infection of primary cervical epithelial cells. Cell Microbiol 4, 571-584.

Elkins, C. and Rest, R.F. (1990). Monoclonal antibodies to outer membrane protein PII block interactions of Neisseria gonorrhoeae with human neutrophils. Infect Immun 58, 1078-1084.

Eschenbach, D.A., Davick, P.R., Williams, B.L., Klebanoff, S.J., Young-Smith, K., Critchlow, C.M. and Holmes, K.K. (1989). Prevalence of hydrogen peroxideproducing Lactobacillus species in normal women and women with bacterial vaginosis. J Clin Microbiol 27, 251-256.

Estabrook, M.M., Zhou, D. and Apicella, M.A. (1998). Nonopsonic phagocytosis of group C Neisseria meningitidis by human neutrophils. Infect Immun 66, 10281036. 
Evans, B.A. (1977). Ultrastructural study of cervical gonorrhea. J Infect Dis 136, 248255.

Fang, F.C. (2004). Antimicrobial reactive oxygen and nitrogen species: concepts and controversies. Nat Rev Microbiol 2, 820-832.

Farrell, C.F. and Rest, R.F. (1990). Up-regulation of human neutrophil receptors for Neisseria gonorrhoeae expressing PII outer membrane proteins. Infect Immun 58, 2777-2784.

Farzadegan, H. and Roth, I.L. (1975). Scanning electron microscopy and freeze-etching of gonorrhoeal urethral exudate. Br J Vener Dis 51, 83-91.

Faurschou, M. and Borregaard, N. (2003). Neutrophil granules and secretory vesicles in inflammation. Microbes Infect 5, 1317-1327.

Feinen, B., Jerse, A.E., Gaffen, S.L. and Russell, M.W. (2010). Critical role of Th17 responses in a murine model of Neisseria gonorrhoeae genital infection. Mucosal Immunol 3, 312-321.

Fensome, A., Cunningham, E., Prosser, S., Tan, S.K., Swigart, P., Thomas, G., et al. (1996). ARF and PITP restore GTP gamma S-stimulated protein secretion from cytosol-depleted HL60 cells by promoting PIP2 synthesis. Curr Biol 6, 730-738.

Fichorova, R.N., Desai, P.J., Gibson, F.C., 3rd and Genco, C.A. (2001). Distinct proinflammatory host responses to Neisseria gonorrhoeae infection in immortalized human cervical and vaginal epithelial cells. Infect Immun 69, 58405848 .

Fischer, S.H. and Rest, R.F. (1988). Gonococci possessing only certain P.II outer membrane proteins interact with human neutrophils. Infect Immun 56, 1574-1579.

Fisette, P.L., Ram, S., Andersen, J.M., Guo, W. and Ingalls, R.R. (2003). The Lip lipoprotein from Neisseria gonorrhoeae stimulates cytokine release and NFkappaB activation in epithelial cells in a Toll-like receptor 2-dependent manner. $J$ Biol Chem 278, 46252-46260.

Frangipane, J.V. and Rest, R.F. (1992). Anaerobic growth of gonococci does not alter their Opa-mediated interactions with human neutrophils. Infect Immun 60, 1793 1799.

Fratti, R.A., Backer, J.M., Gruenberg, J., Corvera, S. and Deretic, V. (2001). Role of phosphatidylinositol 3-kinase and Rab5 effectors in phagosomal biogenesis and mycobacterial phagosome maturation arrest. J Cell Biol 154, 631-644.

Freissler, E., Meyer auf der Heyde, A., David, G., Meyer, T.F. and Dehio, C. (2000). Syndecan-1 and syndecan-4 can mediate the invasion of OpaHSPG-expressing Neisseria gonorrhoeae into epithelial cells. Cell Microbiol 2, 69-82.

Fulcher, N.B. (2004) The role of Neisseria gonorrhoeae opacity proteins in host cell interactions and pathogenesis. Ph.D. thesis. In Department of Microbiology and Immunology. Chapel Hill, NC, University of North Carolina.

Fumagalli, L., Zhang, H., Baruzzi, A., Lowell, C.A. and Berton, G. (2007). The Src family kinases Hck and Fgr regulate neutrophil responses to N-formyl-methionylleucyl-phenylalanine. J Immunol 178, 3874-3885.

Geier, H. and Celli, J. (2011). Phagocytic receptors dictate phagosomal escape and intracellular proliferation of Francisella tularensis. Infect Immun 79, 2204-2214. 
Gilbert, M., Watson, D.C., Cunningham, A.M., Jennings, M.P., Young, N.M. and Wakarchuk, W.W. (1996). Cloning of the lipooligosaccharide alpha-2,3sialyltransferase from the bacterial pathogens Neisseria meningitidis and Neisseria gonorrhoeae. J Biol Chem 271, 28271-28276.

Gill, M.J., McQuillen, D.P., van Putten, J.P., Wetzler, L.M., Bramley, J., Crooke, H., et al. (1996). Functional characterization of a sialyltransferase-deficient mutant of Neisseria gonorrhoeae. Infect Immun 64, 3374-3378.

Gray-Owen, S.D. and Blumberg, R.S. (2006). CEACAM1: contact-dependent control of immunity. Nat Rev Immunol 6, 433-446.

Gray-Owen, S.D., Dehio, C., Haude, A., Grunert, F. and Meyer, T.F. (1997a). CD66 carcinoembryonic antigens mediate interactions between Opa-expressing Neisseria gonorrhoeae and human polymorphonuclear phagocytes. EMBO J 16, 3435-3445.

Gray-Owen, S.D., Lorenzen, D.R., Haude, A., Meyer, T.F. and Dehio, C. (1997b). Differential Opa specificities for CD66 receptors influence tissue interactions and cellular response to Neisseria gonorrhoeae. Mol Microbiol 26, 971-980.

Griffiss, J.M., Jarvis, G.A., O'Brien, J.P., Eads, M.M. and Schneider, H. (1991). Lysis of Neisseria gonorrhoeae initiated by binding of normal human IgM to a hexosamine-containing lipooligosaccharide epitope(s) is augmented by strainspecific, properdin-binding-dependent alternative complement pathway activation. J Immunol 147, 298-305.

Groves, E., Dart, A.E., Covarelli, V. and Caron, E. (2008). Molecular mechanisms of phagocytic uptake in mammalian cells. Cell Mol Life Sci 65, 1957-1976.

Gu, A., Zhang, Z., Zhang, N., Tsark, W. and Shively, J.E. (2010). Generation of human CEACAM1 transgenic mice and binding of Neisseria Opa protein to their neutrophils. PLoS One 5, e10067.

Gulati, S., Cox, A., Lewis, L.A., Michael, F.S., Li, J., Boden, R., et al. (2005). Enhanced factor $\mathrm{H}$ binding to sialylated Gonococci is restricted to the sialylated lacto-Nneotetraose lipooligosaccharide species: implications for serum resistance and evidence for a bifunctional lipooligosaccharide sialyltransferase in Gonococci. Infect Immun 73, 7390-7397.

Gutkind, J.S. and Robbins, K.C. (1989). Translocation of the FGR protein-tyrosine kinase as a consequence of neutrophil activation. Proc Natl Acad Sci U S A 86, 87838787.

Hagman, K.E., Pan, W., Spratt, B.G., Balthazar, J.T., Judd, R.C. and Shafer, W.M. (1995a). Resistance of Neisseria gonorrhoeae to antimicrobial hydrophobic agents is modulated by the mtrRCDE efflux system. Microbiology 141 ( Pt 3), 611-622.

Hagman, K.E. and Shafer, W.M. (1995b). Transcriptional control of the mtr efflux system of Neisseria gonorrhoeae. J Bacteriol 177, 4162-4165.

Haines, K.A., Yeh, L., Blake, M.S., Cristello, P., Korchak, H. and Weissmann, G. (1988). Protein I, a translocatable ion channel from Neisseria gonorrhoeae, selectively inhibits exocytosis from human neutrophils without inhibiting O2-generation. $J$ Biol Chem 263, 945-951.

Hamrick, T.S., Dempsey, J.A., Cohen, M.S. and Cannon, J.G. (2001). Antigenic variation of gonococcal pilin expression in vivo: analysis of the strain FA1090 pilin 
repertoire and identification of the pilS gene copies recombining with pilE during experimental human infection. Microbiology 147, 839-849.

Harvey, H.A., Jennings, M.P., Campbell, C.A., Williams, R. and Apicella, M.A. (2001). Receptor-mediated endocytosis of Neisseria gonorrhoeae into primary human urethral epithelial cells: the role of the asialoglycoprotein receptor. Mol Microbiol 42, 659-672.

Hassett, D.J., Charniga, L. and Cohen, M.S. (1990). recA and catalase in H2O2-mediated toxicity in Neisseria gonorrhoeae. J Bacteriol 172, 7293-7296.

Hauck, C.R. and Meyer, T.F. (2003). 'Small' talk: Opa proteins as mediators of Neisseriahost-cell communication. Curr Opin Microbiol 6, 43-49.

Heckman, K.L. and Pease, L.R. (2007). Gene splicing and mutagenesis by PCR-driven overlap extension. Nat Protoc 2, 924-932.

Hedges, S.R., Sibley, D.A., Mayo, M.S., Hook, E.W., 3rd and Russell, M.W. (1998). Cytokine and antibody responses in women infected with Neisseria gonorrhoeae: effects of concomitant infections. J Infect Dis 178, 742-751.

Hobbs, M.M., Anderson, J.E., Balthazar, J.T., Kandler, J.L., Carlson, R.W., Ganguly, J., et al. (2013). Lipid A's structure mediates Neisseria gonorrhoeae fitness during experimental infection of mice and men. MBio 4, e00892-00813.

Hobbs, M.M., Sparling, P.F., Cohen, M.S., Shafer, W.M., Deal, C.D. and Jerse, A.E. (2011). Experimental Gonococcal Infection in Male Volunteers: Cumulative Experience with Neisseria gonorrhoeae Strains FA1090 and MS11mkC. Front Microbiol 2, 123.

Hoefel, D., Grooby, W.L., Monis, P.T., Andrews, S. and Saint, C.P. (2003). Enumeration of water-borne bacteria using viability assays and flow cytometry: a comparison to culture-based techniques. J Microbiol Methods 55, 585-597.

Hong, C.W., Kim, T.K., Ham, H.Y., Nam, J.S., Kim, Y.H., Zheng, H., et al. (2010). Lysophosphatidylcholine increases neutrophil bactericidal activity by enhancement of azurophil granule-phagosome fusion via glycine.GlyR alpha 2/TRPM2/p38 MAPK signaling. J Immunol 184, 4401-4413.

Huang, C.B., George, B. and Ebersole, J.L. (2010). Antimicrobial activity of n-6, n-7 and n-9 fatty acids and their esters for oral microorganisms. Arch Oral Biol 55, 555560.

James, J.F. and Swanson, J. (1978). Studies on gonococcus infection. XIII. Occurrence of color/opacity colonial variants in clinical cultures. Infect Immun 19, 332-340.

Jerse, A.E. (1999). Experimental gonococcal genital tract infection and opacity protein expression in estradiol-treated mice. Infect Immun 67, 5699-5708.

Jerse, A.E., Cohen, M.S., Drown, P.M., Whicker, L.G., Isbey, S.F., Seifert, H.S. and Cannon, J.G. (1994). Multiple gonococcal opacity proteins are expressed during experimental urethral infection in the male. J Exp Med 179, 911-920.

Jerse, A.E., Sharma, N.D., Simms, A.N., Crow, E.T., Snyder, L.A. and Shafer, W.M. (2003). A gonococcal efflux pump system enhances bacterial survival in a female mouse model of genital tract infection. Infect Immun 71, 5576-5582.

Johansson, L., Rytkonen, A., Bergman, P., Albiger, B., Kallstrom, H., Hokfelt, T., et al. (2003). CD46 in meningococcal disease. Science 301, 373-375. 
Johnson, M.B. and Criss, A.K. (2011). Resistance of Neisseria gonorrhoeae to neutrophils. Front Microbiol 2, 77.

Johnson, M.B. and Criss, A.K. (2013a). Fluorescence microscopy methods for determining the viability of bacteria in association with mammalian cells. $J$ Vis Exp. 79, doi:10.3791/50729.

Johnson, M.B. and Criss, A.K. (2013b). Neisseria gonorrhoeae phagosomes delay fusion with primary granules to enhance bacterial survival inside human neutrophils. Cell Microbiol 15, 1323-1340.

Johnson, S.R., Steiner, B.M., Cruce, D.D., Perkins, G.H. and Arko, R.J. (1993). Characterization of a catalase-deficient strain of Neisseria gonorrhoeae: evidence for the significance of catalase in the biology of N. gonorrhoeae. Infect Immun 61, 1232-1238.

Kallstrom, H., Blackmer Gill, D., Albiger, B., Liszewski, M.K., Atkinson, J.P. and Jonsson, A.B. (2001). Attachment of Neisseria gonorrhoeae to the cellular pilus receptor CD46: identification of domains important for bacterial adherence. Cell Microbiol 3, 133-143.

Kallstrom, H., Liszewski, M.K., Atkinson, J.P. and Jonsson, A.B. (1997). Membrane cofactor protein (MCP or CD46) is a cellular pilus receptor for pathogenic Neisseria. Mol Microbiol 25, 639-647.

Kaparakis, M., Turnbull, L., Carneiro, L., Firth, S., Coleman, H.A., Parkington, H.C., et al. (2010). Bacterial membrane vesicles deliver peptidoglycan to NOD1 in epithelial cells. Cell Microbiol 12, 372-385.

Kaplan, E.L., Chhatwal, G.S. and Rohde, M. (2006). Reduced ability of penicillin to eradicate ingested group A streptococci from epithelial cells: clinical and pathogenetic implications. Clin Infect Dis 43, 1398-1406.

Kellogg, D.S., Jr., Cohen, I.R., Norins, L.C., Schroeter, A.L. and Reising, G. (1968). Neisseria gonorrhoeae. II. Colonial variation and pathogenicity during 35 months in vitro. J Bacteriol 96, 596-605.

Kellogg, D.S., Jr., Peacock, W.L., Jr., Deacon, W.E., Brown, L. and Pirkle, D.I. (1963). Neisseria Gonorrhoeae. I. Virulence Genetically Linked to Clonal Variation. $J$ Bacteriol 85, 1274-1279.

Kim, J.J., Zhou, D., Mandrell, R.E. and Griffiss, J.M. (1992). Effect of exogenous sialylation of the lipooligosaccharide of Neisseria gonorrhoeae on opsonophagocytosis. Infect Immun 60, 4439-4442.

King, G., James, J.F. and Swanson, J. (1978a). Studies on gonococcus infection. XI. Comparison of in vivo and vitro association of Neisseria gonorrhoeae with human neutrophils. J Infect Dis 137, 38-43.

King, G.J. and Swanson, J. (1978b). Studies on gonococcus infection. XV. Identification of surface proteins of Neisseria gonorrhoeae correlated with leukocyte association. Infect Immun 21, 575-584.

Kirchner, M., Heuer, D. and Meyer, T.F. (2005a). CD46-independent binding of neisserial type IV pili and the major pilus adhesin, PilC, to human epithelial cells. Infect Immun 73, 3072-3082. 
Kirchner, M. and Meyer, T.F. (2005b). The PilC adhesin of the Neisseria type IV pilusbinding specificities and new insights into the nature of the host cell receptor. $\mathrm{Mol}$ Microbiol 56, 945-957.

Kirkcaldy, R.D., Ballard, R.C. and Dowell, D. (2011). Gonococcal resistance: are cephalosporins next? Curr Infect Dis Rep 13, 196-204.

Koyasu, S. (2003). The role of PI3K in immune cells. Nat Immunol 4, 313-319.

Kubica, M., Guzik, K., Koziel, J., Zarebski, M., Richter, W., Gajkowska, B., et al. (2008). A potential new pathway for Staphylococcus aureus dissemination: the silent survival of $\mathrm{S}$. aureus phagocytosed by human monocyte-derived macrophages. PLoS One 3, e1409.

Kunz, A.N., Begum, A.A., Wu, H., D'Ambrozio, J.A., Robinson, J.M., Shafer, W.M., et al. (2012). Impact of fluoroquinolone resistance mutations on gonococcal fitness and in vivo selection for compensatory mutations. J Infect Dis 205, 1821-1829.

Kupsch, E.M., Knepper, B., Kuroki, T., Heuer, I. and Meyer, T.F. (1993). Variable opacity (Opa) outer membrane proteins account for the cell tropisms displayed by Neisseria gonorrhoeae for human leukocytes and epithelial cells. EMBO J 12, 641-650.

Lacy, P. (2006). Mechanisms of degranulation in neutrophils. Allergy Asthma Clin Immunol 2, 98-108.

Lacy, P. and Eitzen, G. (2008). Control of granule exocytosis in neutrophils. Front Biosci 13, 5559-5570.

Lammel, C.J., Sweet, R.L., Rice, P.A., Knapp, J.S., Schoolnik, G.K., Heilbron, D.C. and Brooks, G.F. (1985). Antibody-antigen specificity in the immune response to infection with Neisseria gonorrhoeae. J Infect Dis 152, 990-1001.

Le Cabec, V., Calafat, J. and Borregaard, N. (1997). Sorting of the specific granule protein, NGAL, during granulocytic maturation of HL-60 cells. Blood 89, 21132121.

LeCuyer, B.E., Criss, A.K. and Seifert, H.S. (2010). Genetic characterization of the nucleotide excision repair system of Neisseria gonorrhoeae. J Bacteriol 192, 665673.

Lee, E.H. and Shafer, W.M. (1999). The farAB-encoded efflux pump mediates resistance of gonococci to long-chained antibacterial fatty acids. Mol Microbiol 33, 839-845.

Lee, W.L., Harrison, R.E. and Grinstein, S. (2003). Phagocytosis by neutrophils. Microbes Infect 5, 1299-1306.

Lewis, L.A., Choudhury, B., Balthazar, J.T., Martin, L.E., Ram, S., Rice, P.A., et al. (2009). Phosphoethanolamine substitution of lipid A and resistance of Neisseria gonorrhoeae to cationic antimicrobial peptides and complement-mediated killing by normal human serum. Infect Immun 77, 1112-1120.

Lim, J.P. and Gleeson, P.A. (2011). Macropinocytosis: an endocytic pathway for internalising large gulps. Immunol Cell Biol 89, 836-843.

Lin, L., Ayala, P., Larson, J., Mulks, M., Fukuda, M., Carlsson, S.R., et al. (1997). The Neisseria type $2 \operatorname{IgA} 1$ protease cleaves LAMP1 and promotes survival of bacteria within epithelial cells. Mol Microbiol 24, 1083-1094. 
Logan, M.R., Lacy, P., Odemuyiwa, S.O., Steward, M., Davoine, F., Kita, H. and Moqbel, R. (2006). A critical role for vesicle-associated membrane protein-7 in exocytosis from human eosinophils and neutrophils. Allergy 61, 777-784.

Lorenzen, D.R., Gunther, D., Pandit, J., Rudel, T., Brandt, E. and Meyer, T.F. (2000). Neisseria gonorrhoeae porin modifies the oxidative burst of human professional phagocytes. Infect Immun 68, 6215-6222.

Makepeace, B.L., Watt, P.J., Heckels, J.E. and Christodoulides, M. (2001). Interactions of Neisseria gonorrhoeae with mature human macrophage opacity proteins influence production of proinflammatory cytokines. Infect Immun 69, 1909-1913.

Makino, S., van Putten, J.P. and Meyer, T.F. (1991). Phase variation of the opacity outer membrane protein controls invasion by Neisseria gonorrhoeae into human epithelial cells. EMBO J 10, 1307-1315.

Malm, J., Sorensen, O., Persson, T., Frohm-Nilsson, M., Johansson, B., Bjartell, A., et al. (2000). The human cationic antimicrobial protein (hCAP-18) is expressed in the epithelium of human epididymis, is present in seminal plasma at high concentrations, and is attached to spermatozoa. Infect Immun 68, 4297-4302.

Malorny, B., Morelli, G., Kusecek, B., Kolberg, J. and Achtman, M. (1998). Sequence diversity, predicted two-dimensional protein structure, and epitope mapping of neisserial Opa proteins. J Bacteriol 180, 1323-1330.

Mandrell, R.E., Lesse, A.J., Sugai, J.V., Shero, M., Griffiss, J.M., Cole, J.A., et al. (1990). In vitro and in vivo modification of Neisseria gonorrhoeae lipooligosaccharide epitope structure by sialylation. J Exp Med 171, 1649-1664.

Martin-Martin, B., Nabokina, S.M., Blasi, J., Lazo, P.A. and Mollinedo, F. (2000). Involvement of SNAP-23 and syntaxin 6 in human neutrophil exocytosis. Blood 96, 2574-2583.

Martinez, A.N., Lahiri, R., Pittman, T.L., Scollard, D., Truman, R., Moraes, M.O. and Williams, D.L. (2009). Molecular determination of Mycobacterium leprae viability by use of real-time PCR. J Clin Microbiol 47, 2124-2130.

Massari, P., Henneke, P., Ho, Y., Latz, E., Golenbock, D.T. and Wetzler, L.M. (2002). Cutting edge: Immune stimulation by neisserial porins is toll-like receptor 2 and MyD88 dependent. J Immunol 168, 1533-1537.

Massari, P., Ram, S., Macleod, H. and Wetzler, L.M. (2003). The role of porins in neisserial pathogenesis and immunity. Trends Microbiol 11, 87-93.

Mavrogiorgos, N., Mekasha, S., Yang, Y., Kelliher, M.A. and Ingalls, R.R. (2013). Activation of NOD receptors by Neisseria gonorrhoeae modulates the innate immune response. Innate Immun.

McCaw, S.E., Liao, E.H. and Gray-Owen, S.D. (2004). Engulfment of Neisseria gonorrhoeae: revealing distinct processes of bacterial entry by individual carcinoembryonic antigen-related cellular adhesion molecule family receptors. Infect Immun 72, 2742-2752.

McGee, Z.A., Stephens, D.S., Hoffman, L.H., Schlech, W.F., 3rd and Horn, R.G. (1983). Mechanisms of mucosal invasion by pathogenic Neisseria. Rev Infect Dis 5 Suppl 4, S708-714.

McMillan, A., McNeillage, G. and Young, H. (1979). Antibodies to Neisseria gonorrhoeae: a study of the urethral exudates of 232 men. J Infect Dis 140, 89-95. 
Merz, A.J. and So, M. (2000). Interactions of pathogenic neisseriae with epithelial cell membranes. Annu Rev Cell Dev Biol 16, 423-457.

Mocsai, A., Jakus, Z., Vantus, T., Berton, G., Lowell, C.A. and Ligeti, E. (2000). Kinase pathways in chemoattractant-induced degranulation of neutrophils: the role of p38 mitogen-activated protein kinase activated by Src family kinases. J Immunol 164, 4321-4331.

Mocsai, A., Zhou, M., Meng, F., Tybulewicz, V.L. and Lowell, C.A. (2002). Syk is required for integrin signaling in neutrophils. Immunity 16, 547-558.

Mohn, H., Le Cabec, V., Fischer, S. and Maridonneau-Parini, I. (1995). The src-family protein-tyrosine kinase $\mathrm{p} 59 \mathrm{hck}$ is located on the secretory granules in human neutrophils and translocates towards the phagosome during cell activation. Biochem J 309 ( Pt 2), 657-665.

Mollinedo, F., Janssen, H., de la Iglesia-Vicente, J., Villa-Pulgarin, J.A. and Calafat, J. (2010). Selective fusion of azurophilic granules with Leishmania-containing phagosomes in human neutrophils. J Biol Chem 285, 34528-34536.

Mollinedo, F., Martin-Martin, B., Calafat, J., Nabokina, S.M. and Lazo, P.A. (2003). Role of vesicle-associated membrane protein-2, through Q-soluble Nethylmaleimide-sensitive factor attachment protein receptor/R-soluble $\mathrm{N}$ ethylmaleimide-sensitive factor attachment protein receptor interaction, in the exocytosis of specific and tertiary granules of human neutrophils. J Immunol 170, 1034-1042.

Morand, P.C., Bille, E., Morelle, S., Eugene, E., Beretti, J.L., Wolfgang, M., et al. (2004). Type IV pilus retraction in pathogenic Neisseria is regulated by the PilC proteins. EMBO J 23, 2009-2017.

Morse, S.A. and Bartenstein, L. (1980). Purine metabolism in Neisseria gonorrhoeae: the requirement for hypoxanthine. Can J Microbiol 26, 13-20.

Mueller, R.S., McDougald, D., Cusumano, D., Sodhi, N., Kjelleberg, S., Azam, F. and Bartlett, D.H. (2007). Vibrio cholerae strains possess multiple strategies for abiotic and biotic surface colonization. J Bacteriol 189, 5348-5360.

Munafo, D.B., Johnson, J.L., Ellis, B.A., Rutschmann, S., Beutler, B. and Catz, S.D. (2007). Rab27a is a key component of the secretory machinery of azurophilic granules in granulocytes. Biochem J 402, 229-239.

Murphy, G.L., Connell, T.D., Barritt, D.S., Koomey, M. and Cannon, J.G. (1989). Phase variation of gonococcal protein II: regulation of gene expression by slipped-strand mispairing of a repetitive DNA sequence. Cell 56, 539-547.

N'Diaye, E.N., Darzacq, X., Astarie-Dequeker, C., Daffe, M., Calafat, J. and Maridonneau-Parini, I. (1998). Fusion of azurophil granules with phagosomes and activation of the tyrosine kinase Hck are specifically inhibited during phagocytosis of mycobacteria by human neutrophils. J Immunol 161, 4983-4991.

Nassif, X., Beretti, J.L., Lowy, J., Stenberg, P., O'Gaora, P., Pfeifer, J., et al. (1994). Roles of pilin and PilC in adhesion of Neisseria meningitidis to human epithelial and endothelial cells. Proc Natl Acad Sci U S A 91, 3769-3773.

Naucler, C., Grinstein, S., Sundler, R. and Tapper, H. (2002). Signaling to localized degranulation in neutrophils adherent to immune complexes. J Leukoc Biol 71, 701-710. 
Newburger, P.E., Chovaniec, M.E., Greenberger, J.S. and Cohen, H.J. (1979). Functional changes in human leukemic cell line HL-60. A model for myeloid differentiation. J Cell Biol 82, 315-322.

Newcombe, J., Jeynes, J.C., Mendoza, E., Hinds, J., Marsden, G.L., Stabler, R.A., et al. (2005). Phenotypic and transcriptional characterization of the meningococcal PhoPQ system, a magnesium-sensing two-component regulatory system that controls genes involved in remodeling the meningococcal cell surface. $J$ Bacteriol 187, 4967-4975.

O'Hanlon, D.E., Lanier, B.R., Moench, T.R. and Cone, R.A. (2010). Cervicovaginal fluid and semen block the microbicidal activity of hydrogen peroxide produced by vaginal lactobacilli. BMC Infect Dis 10, 120.

Ofek, I., Beachey, E.H. and Bisno, A.L. (1974). Resistance of Neisseria gonorrhoeae to phagocytosis: relationship to colonial morphology and surface pili. J Infect Dis 129, 310-316.

Oh, H., Siano, B. and Diamond, S. (2008). Neutrophil isolation protocol. J Vis Exp.

Ohneck, E.A., Zalucki, Y.M., Johnson, P.J., Dhulipala, V., Golparian, D., Unemo, M., et al. (2011). A novel mechanism of high-level, broad-spectrum antibiotic resistance caused by a single base pair change in Neisseria gonorrhoeae. MBio 2.

Ovcinnikov, N.M. and Delektorskij, V.V. (1971). Electron microscope studies of gonococci in the urethral secretions of patients with gonorrhoea. Br J Vener Dis 47, 419-439.

Pantelic, M., Chen, I., Parker, J., Zhang, P., Grunert, F. and Chen, T. (2004). Retinoic acid treated HL60 cells express CEACAM1 (CD66a) and phagocytose Neisseria gonorrhoeae. FEMS Immunol Med Microbiol 42, 261-266.

Papayannopoulos, V. and Zychlinsky, A. (2009). NETs: a new strategy for using old weapons. Trends Immunol 30, 513-521.

Parsons, N.J., Curry, A., Fox, A.J., Jones, D.M., Cole, J.A. and Smith, H. (1992). The serum resistance of gonococci in the majority of urethral exudates is due to sialylated lipopolysaccharide seen as a surface coat. FEMS Microbiol Lett 69, 295-299.

Penn, C.W., Parsons, N.J., Sen, D., Veale, D.R. and Smith, H. (1977). Immunization of guinea pigs with Neisseria gonorrhoeae: strain specificity and mechanisms of immunity. J Gen Microbiol 100, 159-166.

Perera, N.C., Schilling, O., Kittel, H., Back, W., Kremmer, E. and Jenne, D.E. (2012). NSP4, an elastase-related protease in human neutrophils with arginine specificity. Proc Natl Acad Sci U S A 109, 6229-6234.

Plested, J.S., Makepeace, K., Jennings, M.P., Gidney, M.A., Lacelle, S., Brisson, J., et al. (1999). Conservation and accessibility of an inner core lipopolysaccharide epitope of Neisseria meningitidis. Infect Immun 67, 5417-5426.

Popp, A., Dehio, C., Grunert, F., Meyer, T.F. and Gray-Owen, S.D. (1999). Molecular analysis of neisserial Opa protein interactions with the CEA family of receptors: identification of determinants contributing to the differential specificities of binding. Cell Microbiol 1, 169-181.

Porter, E., Yang, H., Yavagal, S., Preza, G.C., Murillo, O., Lima, H., et al. (2005). Distinct defensin profiles in Neisseria gonorrhoeae and Chlamydia trachomatis 
urethritis reveal novel epithelial cell-neutrophil interactions. Infect Immun 73, 4823-4833.

Potter, A.J., Kidd, S.P., Edwards, J.L., Falsetta, M.L., Apicella, M.A., Jennings, M.P. and McEwan, A.G. (2009). Thioredoxin reductase is essential for protection of Neisseria gonorrhoeae against killing by nitric oxide and for bacterial growth during interaction with cervical epithelial cells. J Infect Dis 199, 227-235.

Pridmore, A.C., Jarvis, G.A., John, C.M., Jack, D.L., Dower, S.K. and Read, R.C. (2003). Activation of toll-like receptor 2 (TLR2) and TLR4/MD2 by Neisseria is independent of capsule and lipooligosaccharide (LOS) sialylation but varies widely among LOS from different strains. Infect Immun 71, 3901-3908.

Qu, X.D., Harwig, S.S., Oren, A.M., Shafer, W.M. and Lehrer, R.I. (1996). Susceptibility of Neisseria gonorrhoeae to protegrins. Infect Immun 64, 1240-1245.

Rahman, M., Kallstrom, H., Normark, S. and Jonsson, A.B. (1997). PilC of pathogenic Neisseria is associated with the bacterial cell surface. Mol Microbiol 25, 11-25.

Ram, S., Cullinane, M., Blom, A.M., Gulati, S., McQuillen, D.P., Monks, B.G., et al. (2001). Binding of C4b-binding protein to porin: a molecular mechanism of serum resistance of Neisseria gonorrhoeae. J Exp Med 193, 281-295.

Ram, S., Lewis, L.A. and Rice, P.A. (2010). Infections of people with complement deficiencies and patients who have undergone splenectomy. Clin Microbiol Rev 23, 740-780.

Ram, S., McQuillen, D.P., Gulati, S., Elkins, C., Pangburn, M.K. and Rice, P.A. (1998a). Binding of complement factor $\mathrm{H}$ to loop 5 of porin protein 1A: a molecular mechanism of serum resistance of nonsialylated Neisseria gonorrhoeae. J Exp Med 188, 671-680.

Ram, S., Sharma, A.K., Simpson, S.D., Gulati, S., McQuillen, D.P., Pangburn, M.K. and Rice, P.A. (1998b). A novel sialic acid binding site on factor $\mathrm{H}$ mediates serum resistance of sialylated Neisseria gonorrhoeae. J Exp Med 187, 743-752.

Ramsey, K.H., Schneider, H., Cross, A.S., Boslego, J.W., Hoover, D.L., Staley, T.L., et al. (1995). Inflammatory cytokines produced in response to experimental human gonorrhea. J Infect Dis 172, 186-191.

Rest, R.F. (1979). Killing of Neisseria gonorrhoeae by human polymorphonuclear neutrophil granule extracts. Infect Immun 25, 574-579.

Rest, R.F., Cooney, M.H. and Spitznagel, J.K. (1977). Susceptibility of lipopolysaccharide mutants to the bactericidal action of human neutrophil lysosomal fractions. Infect Immun 16, 145-151.

Rest, R.F., Fischer, S.H., Ingham, Z.Z. and Jones, J.F. (1982). Interactions of Neisseria gonorrhoeae with human neutrophils: effects of serum and gonococcal opacity on phagocyte killing and chemiluminescence. Infect Immun 36, 737-744.

Rest, R.F. and Frangipane, J.V. (1992). Growth of Neisseria gonorrhoeae in CMP-Nacetylneuraminic acid inhibits nonopsonic (opacity-associated outer membrane protein-mediated) interactions with human neutrophils. Infect Immun 60, 989-997.

Rest, R.F. and Pretzer, E. (1981). Degradation of gonococcal outer membrane proteins by human neutrophil lysosomal proteases. Infect Immun 34, 62-68. 
Rice, P.A. and Kasper, D.L. (1982). Characterization of serum resistance of Neisseria gonorrhoeae that disseminate. Roles of blocking antibody and gonococcal outer membrane proteins. J Clin Invest 70, 157-167.

Rock, J.P. and Rest, R.F. (1988). Rapid damage to membranes of Neisseria gonorrhoeae caused by human neutrophil granule extracts. J Gen Microbiol 134, 509-519.

Roos, D., van Bruggen, R. and Meischl, C. (2003). Oxidative killing of microbes by neutrophils. Microbes Infect 5, 1307-1315.

Rouquette, C., Harmon, J.B. and Shafer, W.M. (1999). Induction of the mtrCDE-encoded efflux pump system of Neisseria gonorrhoeae requires MtrA, an AraC-like protein. Mol Microbiol 33, 651-658.

Rudel, T., Boxberger, H.J. and Meyer, T.F. (1995). Pilus biogenesis and epithelial cell adherence of Neisseria gonorrhoeae pilC double knock-out mutants. Mol Microbiol 17, 1057-1071.

Sadarangani, M., Pollard, A.J. and Gray-Owen, S.D. (2010). Opa proteins and CEACAMs: pathways of immune engagement for pathogenic Neisseria. FEMS Microbiol Rev.

Sadarangani, M., Pollard, A.J. and Gray-Owen, S.D. (2011). Opa proteins \& CEACAMs: pathways of immune engagement for pathogenic Neisseria. FEMS Microbiol Rev.

Saigh, J.H., Sanders, C.C. and Sanders, W.E., Jr. (1978). Inhibition of Neisseria gonorrhoeae by aerobic and facultatively anaerobic components of the endocervical flora: evidence for a protective effect against infection. Infect Immun 19, 704-710.

Salit, I.E. (1982). The differential susceptibility of gonococcal opacity variants to sex hormones. Can J Microbiol 28, 301-306.

Sarafian, S.K., Tam, M.R. and Morse, S.A. (1983). Gonococcal protein I-specific opsonic IgG in normal human serum. J Infect Dis 148, 1025-1032.

Sarantis, H. and Gray-Owen, S.D. (2007). The specific innate immune receptor CEACAM3 triggers neutrophil bactericidal activities via a Syk kinase-dependent pathway. Cell Microbiol 9, 2167-2180.

Sarantis, H. and Gray-Owen, S.D. (2012). Defining the roles of human carcinoembryonic antigen-related cellular adhesion molecules during neutrophil responses to Neisseria gonorrhoeae. Infect Immun 80, 345-358.

Schook, P.O., Stohl, E.A., Criss, A.K. and Seifert, H.S. (2011). The DNA-binding activity of the Neisseria gonorrhoeae LexA orthologue NG1427 is modulated by oxidation. Mol Microbiol 79, 846-860.

Schwalbe, R.S., Sparling, P.F. and Cannon, J.G. (1985). Variation of Neisseria gonorrhoeae protein II among isolates from an outbreak caused by a single gonococcal strain. Infect Immun 49, 250-252.

Segal, A.W. (2005). How neutrophils kill microbes. Annu Rev Immunol 23, 197-223.

Seib, K.L., Simons, M.P., Wu, H.J., McEwan, A.G., Nauseef, W.M., Apicella, M.A. and Jennings, M.P. (2005). Investigation of oxidative stress defenses of Neisseria gonorrhoeae by using a human polymorphonuclear leukocyte survival assay. Infect Immun 73, 5269-5272.

Seib, K.L., Tseng, H.J., McEwan, A.G., Apicella, M.A. and Jennings, M.P. (2004). Defenses against oxidative stress in Neisseria gonorrhoeae and Neisseria 
meningitidis: distinctive systems for different lifestyles. J Infect Dis 190, 136147.

Seib, K.L., Wu, H.J., Kidd, S.P., Apicella, M.A., Jennings, M.P. and McEwan, A.G. (2006). Defenses against oxidative stress in Neisseria gonorrhoeae: a system tailored for a challenging environment. Microbiol Mol Biol Rev 70, 344-361.

Seifert, H.S., Wright, C.J., Jerse, A.E., Cohen, M.S. and Cannon, J.G. (1994). Multiple gonococcal pilin antigenic variants are produced during experimental human infections. J Clin Invest 93, 2744-2749.

Sengelov, H., Kjeldsen, L. and Borregaard, N. (1993). Control of exocytosis in early neutrophil activation. J Immunol 150, 1535-1543.

Shafer, W.M. (1988). Lipopolysaccharide masking of gonococcal outer-membrane proteins modulates binding of bacterial cathepsin $\mathrm{G}$ to gonococci. J Gen Microbiol 134, 539-545.

Shafer, W.M. and Morse, S.A. (1987). Cleavage of the protein III and major ironregulated protein of Neisseria gonorrhoeae by lysosomal cathepsin G. J Gen Microbiol 133, 155-162.

Shafer, W.M., Onunka, V. and Hitchcock, P.J. (1986a). A spontaneous mutant of Neisseria gonorrhoeae with decreased resistance to neutrophil granule proteins. $J$ Infect Dis 153, 910-917.

Shafer, W.M., Onunka, V.C., Jannoun, M. and Huthwaite, L.W. (1990). Molecular mechanism for the antigonococcal action of lysosomal cathepsin G. Mol Microbiol 4, 1269-1277.

Shafer, W.M., Onunka, V.C. and Martin, L.E. (1986b). Antigonococcal activity of human neutrophil cathepsin G. Infect Immun 54, 184-188.

Shafer, W.M., Qu, X., Waring, A.J. and Lehrer, R.I. (1998). Modulation of Neisseria gonorrhoeae susceptibility to vertebrate antibacterial peptides due to a member of the resistance/nodulation/division efflux pump family. Proc Natl Acad Sci U S A 95, 1829-1833.

Shafer, W.M. and Rest, R.F. (1989). Interactions of gonococci with phagocytic cells. Annu Rev Microbiol 43, 121-145.

Shen, C., Luo, Y., Nou, X., Bauchan, G., Zhou, B., Wang, Q. and Millner, P. (2012). Enhanced inactivation of Salmonella and Pseudomonas biofilms on stainless steel by use of T-128, a fresh-produce washing aid, in chlorinated wash solutions. Appl Environ Microbiol 78, 6789-6798.

Siegel, M., Olsen, D., Critchlow, C. and Buchanan, T.M. (1982). Gonococcal pili: safety and immunogenicity in humans and antibody function in vitro. J Infect Dis 145, 300-310.

Silliman, C.C., Elzi, D.J., Ambruso, D.R., Musters, R.J., Hamiel, C., Harbeck, R.J., et al. (2003). Lysophosphatidylcholines prime the NADPH oxidase and stimulate multiple neutrophil functions through changes in cytosolic calcium. J Leukoc Biol 73, 511-524.

Simms, A.N. and Jerse, A.E. (2006). In vivo selection for Neisseria gonorrhoeae opacity protein expression in the absence of human carcinoembryonic antigen cell adhesion molecules. Infect Immun 74, 2965-2974. 
Simons, M.P., Nauseef, W.M. and Apicella, M.A. (2005). Interactions of Neisseria gonorrhoeae with adherent polymorphonuclear leukocytes. Infect Immun 73, 1971-1977.

Simons, M.P., Nauseef, W.M., Griffith, T.S. and Apicella, M.A. (2006). Neisseria gonorrhoeae delays the onset of apoptosis in polymorphonuclear leukocytes. Cell Microbiol 8, 1780-1790.

Simpson, K.L., Jones, A., Norman, S. and Holmes, C.H. (1997). Expression of the complement regulatory proteins decay accelerating factor (DAF, CD55), membrane cofactor protein (MCP, CD46) and CD59 in the normal human uterine cervix and in premalignant and malignant cervical disease. Am J Pathol 151, 1455-1467.

Skaar, E.P., Tobiason, D.M., Quick, J., Judd, R.C., Weissbach, H., Etienne, F., et al. (2002). The outer membrane localization of the Neisseria gonorrhoeae MsrA/B is involved in survival against reactive oxygen species. Proc Natl Acad Sci U S A 99, 10108-10113.

Smirnov, A., Daily, K.P. and Criss, A.K. (2013). Assembly of NADPH oxidase in human neutrophils is modulated by the opacity-associated protein expression state of Neisseria gonorrhoeae. Infect Immun.

Smith, C.D., Berk, S.G., Brandl, M.T. and Riley, L.W. (2012). Survival characteristics of diarrheagenic Escherichia coli pathotypes and Helicobacter pylori during passage through the free-living ciliate, Tetrahymena sp. FEMS Microbiol Ecol 82, 574583.

Soderholm, N., Vielfort, K., Hultenby, K. and Aro, H. (2011). Pathogenic Neisseria Hitchhike on the Uropod of Human Neutrophils. PLoS One 6, e24353.

Soler-Garcia, A.A. and Jerse, A.E. (2004). A Neisseria gonorrhoeae catalase mutant is more sensitive to hydrogen peroxide and paraquat, an inducer of toxic oxygen radicals. Microb Pathog 37, 55-63.

Soler-Garcia, A.A. and Jerse, A.E. (2007). Neisseria gonorrhoeae catalase is not required for experimental genital tract infection despite the induction of a localized neutrophil response. Infect Immun 75, 2225-2233.

Song, W., Ma, L., Chen, R. and Stein, D.C. (2000). Role of lipooligosaccharide in Opaindependent invasion of Neisseria gonorrhoeae into human epithelial cells. $J$ Exp Med 191, 949-960.

Sorensen, O.E., Follin, P., Johnsen, A.H., Calafat, J., Tjabringa, G.S., Hiemstra, P.S. and Borregaard, N. (2001). Human cathelicidin, hCAP-18, is processed to the antimicrobial peptide LL-37 by extracellular cleavage with proteinase 3. Blood 97, 3951-3959.

Spence, J.M. and Clark, V.L. (2000). Role of ribosomal protein L12 in gonococcal invasion of Hec1B cells. Infect Immun 68, 5002-5010.

St Amant, D.C., Valentin-Bon, I.E. and Jerse, A.E. (2002). Inhibition of Neisseria gonorrhoeae by Lactobacillus species that are commonly isolated from the female genital tract. Infect Immun 70, 7169-7171.

Staali, L., Bauer, S., Morgelin, M., Bjorck, L. and Tapper, H. (2006). Streptococcus pyogenes bacteria modulate membrane traffic in human neutrophils and 
selectively inhibit azurophilic granule fusion with phagosomes. Cell Microbiol $\mathbf{8}$, 690-703.

Stern, A., Brown, M., Nickel, P. and Meyer, T.F. (1986). Opacity genes in Neisseria gonorrhoeae: control of phase and antigenic variation. Cell 47, 61-71.

Stocks, S.M. (2004). Mechanism and use of the commercially available viability stain, BacLight. Cytometry A 61, 189-195.

Stohl, E.A., Chan, Y.A., Hackett, K.T., Kohler, P.L., Dillard, J.P. and Seifert, H.S. (2012). Neisseria gonorrhoeae virulence factor NG1686 is a bifunctional M23B family metallopeptidase that influences resistance to hydrogen peroxide and colony morphology. J Biol Chem 287, 11222-11233.

Stohl, E.A., Criss, A.K. and Seifert, H.S. (2005). The transcriptome response of Neisseria gonorrhoeae to hydrogen peroxide reveals genes with previously uncharacterized roles in oxidative damage protection. Mol Microbiol 58, 520-532.

Stohl, E.A., Dale, E.M., Criss, A.K. and Seifert, H.S. (2013). Neisseria gonorrhoeae metalloprotease NGO1686 is required for full piliation, and piliation is required for resistance to $\mathrm{H} 2 \mathrm{O} 2-$ and neutrophil-mediated killing. MBio 4.

Stohl, E.A. and Seifert, H.S. (2001). The recX gene potentiates homologous recombination in Neisseria gonorrhoeae. Mol Microbiol 40, 1301-1310.

Stohl, E.A. and Seifert, H.S. (2006). Neisseria gonorrhoeae DNA recombination and repair enzymes protect against oxidative damage caused by hydrogen peroxide. $J$ Bacteriol 188, 7645-7651.

Stuart, L.M. and Ezekowitz, R.A. (2005). Phagocytosis: elegant complexity. Immunity 22, 539-550.

Swanson, J. (1978). Studies on gonococcus infection. XIV. Cell wall protein differences among color/opacity colony variants of Neisseria gonorrhoeae. Infect Immun 21, 292-302.

Swanson, J., Barrera, O., Sola, J. and Boslego, J. (1988). Expression of outer membrane protein II by gonococci in experimental gonorrhea. $J$ Exp Med 168, 2121-2129.

Swanson, J., Robbins, K., Barrera, O., Corwin, D., Boslego, J., Ciak, J., et al. (1987). Gonococcal pilin variants in experimental gonorrhea. J Exp Med 165, 1344-1357.

Swanson, J.E., S., Zeligs, B., Siam, M. A., and Parrott, C. (1974). Studies on Gonococcus Infection. Infection And Immunity 10, 633-644.

Swords, W.E., Guenthner, P.C., Birkness, K.A., Lal, R.B., Dezzutti, C.S. and Quinn, F.D. (2006). Mycobacterium xenopi multiplies within human macrophages and enhances HIV replication in vitro. Microb Pathog 40, 41-47.

Tamilselvam, B., Almeida, R.A., Dunlap, J.R. and Oliver, S.P. (2006). Streptococcus uberis internalizes and persists in bovine mammary epithelial cells. Microb Pathog 40, 279-285.

Tapsall, J.W. (2009). Neisseria gonorrhoeae and emerging resistance to extended spectrum cephalosporins. Curr Opin Infect Dis 22, 87-91.

Thomas, D.W., Hill, J. C., and Tyeryar, F. J. (1973). Interaction of Gonococci with Phagocytic Leukocytes from Men and Mice. Infection And Immunity 8, 98-104.

Thulin, P., Johansson, L., Low, D.E., Gan, B.S., Kotb, M., McGeer, A. and NorrbyTeglund, A. (2006). Viable group A streptococci in macrophages during acute soft tissue infection. PLoS Med 3, e53. 
Tjabringa, G.S., Vos, J.B., Olthuis, D., Ninaber, D.K., Rabe, K.F., Schalkwijk, J., et al. (2005). Host defense effector molecules in mucosal secretions. FEMS Immunol Med Microbiol 45, 151-158.

Tobiason, D.M. and Seifert, H.S. (2001). Inverse relationship between pilus-mediated gonococcal adherence and surface expression of the pilus receptor, CD46. Microbiology 147, 2333-2340.

Tramont, E.C., Ciak, J., Boslego, J., McChesney, D.G., Brinton, C.C. and Zollinger, W. (1980). Antigenic specificity of antibodies in vaginal secretions during infection with Neisseria gonorrhoeae. J Infect Dis 142, 23-31.

Tseng, H.J., McEwan, A.G., Apicella, M.A. and Jennings, M.P. (2003). OxyR acts as a repressor of catalase expression in Neisseria gonorrhoeae. Infect Immun 71, 550556.

Tseng, H.J., Srikhanta, Y., McEwan, A.G. and Jennings, M.P. (2001). Accumulation of manganese in Neisseria gonorrhoeae correlates with resistance to oxidative killing by superoxide anion and is independent of superoxide dismutase activity. Mol Microbiol 40, 1175-1186.

Turner, S., Reid, E., Smith, H. and Cole, J. (2003). A novel cytochrome c peroxidase from Neisseria gonorrhoeae: a lipoprotein from a Gram-negative bacterium. Biochem J 373, 865-873.

Tzeng, Y.L., Ambrose, K.D., Zughaier, S., Zhou, X., Miller, Y.K., Shafer, W.M. and Stephens, D.S. (2005). Cationic antimicrobial peptide resistance in Neisseria meningitidis. J Bacteriol 187, 5387-5396.

Tzeng, Y.L., Kahler, C.M., Zhang, X. and Stephens, D.S. (2008). MisR/MisS twocomponent regulon in Neisseria meningitidis. Infect Immun 76, 704-716.

Urban, C.F., Lourido, S. and Zychlinsky, A. (2006). How do microbes evade neutrophil killing? Cell Microbiol 8, 1687-1696.

van der Ley, P. (1988). Three copies of a single protein II-encoding sequence in the genome of Neisseria gonorrhoeae JS3: evidence for gene conversion and gene duplication. Mol Microbiol 2, 797-806.

van Putten, J.P. and Paul, S.M. (1995). Binding of syndecan-like cell surface proteoglycan receptors is required for Neisseria gonorrhoeae entry into human mucosal cells. EMBO J 14, 2144-2154.

van Ulsen, P. and Tommassen, J. (2006). Protein secretion and secreted proteins in pathogenic Neisseriaceae. FEMS Microbiol Rev 30, 292-319.

van Vliet, S.J., Steeghs, L., Bruijns, S.C., Vaezirad, M.M., Snijders Blok, C., Arenas Busto, J.A., et al. (2009). Variation of Neisseria gonorrhoeae lipooligosaccharide directs dendritic cell-induced T helper responses. PLoS Pathog 5, e1000625.

Van Ziffle, J.A. and Lowell, C.A. (2009). Neutrophil-specific deletion of Syk kinase results in reduced host defense to bacterial infection. Blood 114, 4871-4882.

Vandeputte-Rutten, L., Bos, M.P., Tommassen, J. and Gros, P. (2003). Crystal structure of Neisserial surface protein A (NspA), a conserved outer membrane protein with vaccine potential. J Biol Chem 278, 24825-24830.

Veal, W.L., Nicholas, R.A. and Shafer, W.M. (2002). Overexpression of the MtrC-MtrDMtrE efflux pump due to an mtrR mutation is required for chromosomally 
mediated penicillin resistance in Neisseria gonorrhoeae. J Bacteriol 184, 56195624.

Veale, D.R., Finch, H. and Smith, H. (1976). Penetration of penicillin into human phagocytes containing Neisseria gonorrhoeae: intracellular survival and growth at optimum concentrations of antibiotic. J Gen Microbiol 96, 353-363.

Veale, D.R., Goldner, M., Penn, C.W., Ward, J. and Smith, H. (1979). The intracellular survival and growth of gonococci in human phagocytes. J Gen Microbiol 113, 383-393.

Virji, M. (2009). Pathogenic neisseriae: surface modulation, pathogenesis and infection control. Nat Rev Microbiol 7, 274-286.

Virji, M. and Heckels, J.E. (1986). The effect of protein II and pili on the interaction of Neisseria gonorrhoeae with human polymorphonuclear leucocytes. J Gen Microbiol 132, 503-512.

Virji, M., Makepeace, K., Ferguson, D.J. and Watt, S.M. (1996a). Carcinoembryonic antigens (CD66) on epithelial cells and neutrophils are receptors for Opa proteins of pathogenic neisseriae. Mol Microbiol 22, 941-950.

Virji, M., Watt, S.M., Barker, S., Makepeace, K. and Doyonnas, R. (1996b). The Ndomain of the human CD66a adhesion molecule is a target for Opa proteins of Neisseria meningitidis and Neisseria gonorrhoeae. Mol Microbiol 22, 929-939.

Virji, M.a.H., J. E. (1986). The Effect of Protein II and Pili on the Interaction of Neisseria gonorrhoeae with Human Polymorphonuclear Leucocytes. General Microbiology 132, 503-512.

Voyich, J.M., Braughton, K.R., Sturdevant, D.E., Whitney, A.R., Said-Salim, B., Porcella, S.F., et al. (2005). Insights into mechanisms used by Staphylococcus aureus to avoid destruction by human neutrophils. J Immunol 175, 3907-3919.

Waage, A., Halstensen, A., Shalaby, R., Brandtzaeg, P., Kierulf, P. and Espevik, T. (1989). Local production of tumor necrosis factor alpha, interleukin 1, and interleukin 6 in meningococcal meningitis. Relation to the inflammatory response. J Exp Med 170, 1859-1867.

Wang, J., Gray-Owen, S.D., Knorre, A., Meyer, T.F. and Dehio, C. (1998). Opa binding to cellular CD66 receptors mediates the transcellular traversal of Neisseria gonorrhoeae across polarized T84 epithelial cell monolayers. Mol Microbiol 30, 657-671.

Warner, D.M., Shafer, W.M. and Jerse, A.E. (2008). Clinically relevant mutations that cause derepression of the Neisseria gonorrhoeae MtrC-MtrD-MtrE Efflux pump system confer different levels of antimicrobial resistance and in vivo fitness. $\mathrm{Mol}$ Microbiol 70, 462-478.

Werner, E. (2004). GTPases and reactive oxygen species: switches for killing and signaling. J Cell Sci 117, 143-153.

Wiesner, P.J. and Thompson, S.E., 3rd (1980). Gonococcal diseases. Dis Mon 26, 1-44.

Wilks, K.E., Dunn, K.L., Farrant, J.L., Reddin, K.M., Gorringe, A.R., Langford, P.R. and Kroll, J.S. (1998). Periplasmic superoxide dismutase in meningococcal pathogenicity. Infect Immun 66, 213-217. 
Witt, K., Veale, D.R., Finch, H., Penn, C.W., Sen, D. and Smith, H. (1976). Resistance of Neisseria gonorrhoeae grown in vivo to ingestion and digestion by phagocytes of human blood. J Gen Microbiol 96, 341-350.

Wolfgang, M., van Putten, J.P., Hayes, S.F., Dorward, D. and Koomey, M. (2000). Components and dynamics of fiber formation define a ubiquitous biogenesis pathway for bacterial pili. EMBO J 19, 6408-6418.

Woolard, M.D. and Frelinger, J.A. (2008). Outsmarting the host: bacteria modulating the immune response. Immunol Res 41, 188-202.

Workowski, K.A. and Berman, S. (2010). Sexually transmitted diseases treatment guidelines, 2010. MMWR Recomm Rep 59, 1-110.

World Health Organization (2012) Global incidence and prevalence of selected curable sexually transmitted infections - 2008. Geneva, Switzerland WHO, Department of Reproductive Health and Research.

Wright, C.J., Jerse, A.E., Cohen, M.S., Cannon, J.G. and Seifert, H.S. (1994). Nonrepresentative PCR amplification of variable gene sequences in clinical specimens containing dilute, complex mixtures of microorganisms. J Clin Microbiol 32, 464-468.

Wu, H. and Jerse, A.E. (2006a). Alpha-2,3-sialyltransferase enhances Neisseria gonorrhoeae survival during experimental murine genital tract infection. Infect Immun 74, 4094-4103.

Wu, H., Soler-Garcia, A.A. and Jerse, A.E. (2009). A strain-specific catalase mutation and mutation of the metal-binding transporter gene mntC attenuate Neisseria gonorrhoeae in vivo but not by increasing susceptibility to oxidative killing by phagocytes. Infect Immun 77, 1091-1102.

Wu, H.J., Seib, K.L., Edwards, J.L., Apicella, M.A., McEwan, A.G. and Jennings, M.P. (2005). Azurin of pathogenic Neisseria spp. is involved in defense against hydrogen peroxide and survival within cervical epithelial cells. Infect Immun 73, 8444-8448.

Wu, H.J., Seib, K.L., Srikhanta, Y.N., Kidd, S.P., Edwards, J.L., Maguire, T.L., et al. (2006b). PerR controls Mn-dependent resistance to oxidative stress in Neisseria gonorrhoeae. Mol Microbiol 60, 401-416.

Yang, L., Wu, L., Zhu, S., Long, Y., Hang, W. and Yan, X. (2010). Rapid, absolute, and simultaneous quantification of specific pathogenic strain and total bacterial cells using an ultrasensitive dual-color flow cytometer. Anal Chem 82, 1109-1116.

Zen, K. and Parkos, C.A. (2003). Leukocyte-epithelial interactions. Curr Opin Cell Biol 15, 557-564.

Zheng, H.Y., Alcorn, T.M. and Cohen, M.S. (1994). Effects of H2O2-producing lactobacilli on Neisseria gonorrhoeae growth and catalase activity. J Infect Dis 170, 1209-1215.

Zhu, W., Chen, C.J., Thomas, C.E., Anderson, J.E., Jerse, A.E. and Sparling, P.F. (2011). Vaccines for gonorrhea: can we rise to the challenge? Front Microbiol 2, 124.

Zughaier, S.M., Tzeng, Y.L., Zimmer, S.M., Datta, A., Carlson, R.W. and Stephens, D.S. (2004). Neisseria meningitidis lipooligosaccharide structure-dependent activation of the macrophage CD14/Toll-like receptor 4 pathway. Infect Immun 72, 371-380. 
